Supporting Information

\title{
Redox-Active, Boron-Based Ligands in Iron Complexes with Inverted Hydride Reactivity in Dehydrogenation Catalysis
}

Andreas Bäcker, ${ }^{\mathrm{a}}$ Yinwu Li, ${ }^{\mathrm{b}}$ Maximilian Fritz, ${ }^{\mathrm{a}}$ Maik Grätz, ${ }^{\mathrm{a}}$ Zhuofeng $\mathrm{Ke}^{\mathrm{b}, *}$ and Robert Langer*,a

1. Experimental Details

2. NMR spectra

3. Variable Temperature NMR Measurements

4. X-Ray Crystallography

5. DFT Calculations

${ }^{2}$ Department of Chemistry, Philipps-Universität Marburg, Hans-Meerwein-Str., 35043 Marburg, Germany, Fax: (+)49-6421-2825617, E-mail: robert.langer@chemie.uni-marburg.de

bSchool of Materials Science and Engineering, PCFM Lab, Sun Yat-Sen University Guangzhou 510275, China; E-mail kezhf3@ mail.sysu.edu.cn. 


\section{Experimental Details}

\subsection{Material and Methods}

All experiments were carried out under an atmosphere of purified argon in a MBraun Labmaster glove box or using standard Schlenk techniques. THF and $\mathrm{C}_{6} \mathrm{D}_{6}$ were dried and distilled from $\mathrm{Na} / \mathrm{K}$ alloy and stored over molecular sieves. Toluene was dried and distilled from sodium and stored over molecular sieves. $n$-Hexane was dried and distilled from LiAlH and stored over molecular sieves. Bis(diphenylphosphino)amine ${ }^{1}$ (dppa) and bis(diphenylphosphino)methan $(\mathrm{dppm})^{2}$ were prepared according to a previously reported procedures.

${ }^{1} \mathrm{H},{ }^{13} \mathrm{C},{ }^{31} \mathrm{P}$ and ${ }^{11} \mathrm{~B}$ NMR spectra were recorded using Bruker DRX 400, DRX 500 and Avance 500 NMR spectrometers. ${ }^{1} \mathrm{H}$ and ${ }^{13} \mathrm{C}\left\{{ }^{1} \mathrm{H}\right\},{ }^{13} \mathrm{C}$-APT (attached proton test) NMR chemical shifts are reported in ppm downfield from tetramethylsilane. The resonance of the residual protons in the deuterated solvent was used as internal standard for ${ }^{1} \mathrm{H}$ NMR. The solvent peak of the deuterated solvent was used as internal standard for ${ }^{13} \mathrm{C}$ NMR. ${ }^{31} \mathrm{P}$ NMR chemical shifts are reported in ppm downfield from $\mathrm{H}_{3} \mathrm{PO}_{4}$ and referenced to an external $85 \%$ solution of phosphoric acid in $\mathrm{D}_{2} \mathrm{O} .{ }^{11} \mathrm{~B} \mathrm{NMR}$ chemical shifts are reported in ppm downfield from $\mathrm{BF}_{3} \cdot \mathrm{Et}_{2} \mathrm{O}$ and referenced to an external solution of $\mathrm{BF}_{3} \cdot \mathrm{Et}_{2} \mathrm{O}$ in $\mathrm{CDCl}_{3}$. The following abbreviations are used for the description of NMR data: br (broad), s (singlet), d (doublet), t (triplet), q (quartet), quin (quintet), sex(sextet), sept (septet), m (multiplet), v (virtual).

FT-IR spectra were recorded by attenuated total reflection of the solid samples on a Bruker Tensor IF37 spectrometer. The intensity of the absorption band is indicated as vw (very weak), w (weak), m (medium), s (strong), vs (very strong) and br (broad).

HR-ESI mass spectra were acquired with a LTQ-FT mass spectrometer (Thermo Fisher Scientific). The resolution was set to 100.000 . 
Synthesis bis(diethylphosphino)amine (depa) $\mathrm{Et}_{2} \mathrm{PCl}(3.5 \mathrm{~mL}, 28.7 \mathrm{mmol})$ is dissolved in toluene $(20 \mathrm{~mL})$ and $\mathrm{NH}_{3}$ gas is passed over the solution for 30 minutes, resulting in the precipitation of a white solid. The reaction mixture was filtered and the precipitate was washed three times with toluene $(3 \times 5 \mathrm{~mL})$. All volatiles of the combined filtrate were removed in vacuo, yielding depa (1.44 g, $7.45 \mathrm{mmol}, 52 \%)$ as a colorless oil, which starts to crystalize in long needles after some time.

${ }^{1} \mathrm{H}-\mathrm{NMR}\left(300.1 \mathrm{MHz}, \mathrm{C}_{6} \mathrm{D}_{6}, 27^{\circ} \mathrm{C}\right) \delta=1.77$ (s, br, $\left.1 \mathrm{H}, \mathrm{NH}\right), 1.34$ (quin, $J=7.6 \mathrm{~Hz}, 4 \mathrm{H}, \mathrm{CH}_{2}$ ), 1.31-1.18 (m, 4H, CH ), 1.0 (quin, $\left.J=7.6 \mathrm{~Hz}, 12 \mathrm{H}, \mathrm{CH}_{3}\right) \mathrm{ppm} .{ }^{13} \mathrm{C}\left\{{ }^{1} \mathrm{H}\right\}-\mathrm{NMR}(75.1 \mathrm{MHz}$, $\left.\mathrm{C}_{6} \mathrm{D}_{6}, 27{ }^{\circ} \mathrm{C}\right) \delta=25.7\left(\mathrm{vt},{ }^{1} J_{\mathrm{CP}}=2.9 \mathrm{~Hz}, 4 \mathrm{C}, \mathrm{CH}_{2}\right), 9.0\left(\mathrm{vt},{ }^{2} J_{\mathrm{CP}}=7.5 \mathrm{~Hz}, 4 \mathrm{C},\left(\mathrm{CH}_{3}\right)_{4}\right) \mathrm{ppm}$. ${ }^{31} \mathrm{P}\left\{{ }^{1} \mathrm{H}\right\}-\mathrm{NMR}\left(121.5 \mathrm{MHz}, \mathrm{C}_{6} \mathrm{D}_{6}, 27^{\circ} \mathrm{C}\right) \delta=43.0(\mathrm{~s}, 2 \mathrm{P}, P-\mathrm{N}-P) \mathrm{ppm}$.

Synthesis of [ $\left.\mathbf{H}_{2} \mathbf{B}(\mathbf{d p p a})_{2}\right] \mathbf{B r}$ (1a) $d p p a(1.1 \mathrm{~g}, 2.85 \mathrm{mmol})$ was dissolved in toluene $(20 \mathrm{~mL})$. After cooling to $-78{ }^{\circ} \mathrm{C}$ a solution of $\mathrm{BH}_{2} \mathrm{Br} \cdot \mathrm{SMe}_{2}$ in $\mathrm{CH}_{2} \mathrm{Cl}_{2}(1 \mathrm{M}, 1.42 \mathrm{~mL}, 1.42 \mathrm{mmol})$ was added slowly. The reaction was warmed to room temperature overnight and the ${ }^{31} \mathrm{P}\left\{{ }^{1} \mathrm{H}\right\}$-NMR spectrum of the reaction mixture indicated full conversion of dppa and the formation of approx.. $65 \%$ of $\left[\mathrm{H}_{2} \mathrm{~B}(\mathrm{dppa})_{2}\right] \mathrm{Br}$. The solvent was removed under reduced pressure and the residue was washed with $\mathrm{THF} / n$-hexane $(1: 1,3 \times 5 \mathrm{~mL})$. All volatiles were removed under high vacuum to obtain $\left[\mathrm{H}_{2} \mathrm{~B}(\mathrm{dppa})_{2}\right] \mathrm{Br}(430 \mathrm{mg}, 0.50 \mathrm{mmol}, 35 \%)$ as a colorless solid.

${ }^{1} \mathrm{H}-\mathrm{NMR}\left(300 \mathrm{MHz}, \mathrm{d}_{8}\right.$-THF) $\delta=7.08-7.2(\mathrm{~m}, 16 \mathrm{H}, \mathrm{CH}), 7.2(\mathrm{~m}, 4 \mathrm{H}, \mathrm{CH}), 7.34\left(\mathrm{t},{ }^{3} J_{\mathrm{HH}}=\right.$ $7.5 \mathrm{~Hz}, 4 \mathrm{H}, \mathrm{CH}), 7.42\left(\mathrm{dd},{ }^{3} J_{\mathrm{HH}}=8.1 \mathrm{~Hz},{ }^{3} J_{\mathrm{HP}}=10.4 \mathrm{~Hz}, 8 \mathrm{H}, \mathrm{CH}\right), 7.68\left(\mathrm{td},{ }^{3} J_{\mathrm{HH}}=8.2 \mathrm{~Hz},{ }^{4} J_{\mathrm{HH}}=\right.$ $1.3 \mathrm{~Hz}, 8 \mathrm{H}, \mathrm{CH}), 9.47$ (m, 2H, NH) ppm. $\left(\mathrm{BH}_{3}\right.$-protons were not detected in $\mathrm{d}^{8}$-THF. Only resonances whose multiplicity is changing upon decoupling are reported in the ${ }^{1} \mathrm{H}\left\{{ }^{31} \mathrm{P}\right\}$ and ${ }^{1} \mathrm{H}\left\{{ }^{11} \mathrm{~B}\right\}$ NMR spectra. ${ }^{1} \mathrm{H}\left\{{ }^{11} \mathrm{~B}\right\}-\mathrm{NMR}(300 \mathrm{MHz}, \mathrm{d} 8-\mathrm{THF}) \delta=2.94\left(\mathrm{t},{ }^{2} \mathrm{JP}_{\mathrm{HP}}=19.9 \mathrm{~Hz}, 2 \mathrm{H}\right.$, B $\left.H_{2}\right)$ ppm. ${ }^{1} \mathrm{H}\left\{{ }^{31} \mathrm{P}\right\}-\mathrm{NMR}\left(300 \mathrm{MHz}, \mathrm{d}_{8}-\mathrm{THF}\right) \delta=7.42\left(\mathrm{~d},{ }^{3} J_{\mathrm{HH}}=8.3,8 \mathrm{H}, \mathrm{CH}\right), 7.68\left(\mathrm{~d},{ }^{3} J_{\mathrm{HH}}=\right.$ $\left.8.2 \mathrm{~Hz},{ }^{4} \mathrm{~J}_{\mathrm{HH}}=1.3 \mathrm{~Hz}, 8 \mathrm{H}, \mathrm{CH}\right), 9.47$ (br, 2H, NH) ppm. ${ }^{11} \mathrm{~B}\left\{{ }^{1} \mathrm{H}\right\}-\mathrm{NMR}\left(96 \mathrm{MHz}, \mathrm{d}_{8}-\mathrm{THF}\right) \delta=$ -30.9 (br t, 1B, B) ppm. ${ }^{31} \mathrm{P}\left\{{ }^{1} \mathrm{H}\right\}-\mathrm{NMR}(122 \mathrm{MHz}, \mathrm{d} 8-\mathrm{THF}) \delta=37.8$ (d, ${ }^{3} J_{\mathrm{PP}}, 2 \mathrm{P}, \mathrm{BPN} P$ ), 41.3 (bs, 2P, BPN) ppm. ${ }^{13} \mathrm{C}-\mathrm{NMR}\left(76 \mathrm{MHz}, \mathrm{d} 8\right.$-THF) $\delta=128.5\left(\mathrm{~d},{ }^{2} J_{\mathrm{CP}}=8.0 \mathrm{~Hz}, 8 \mathrm{C}, C \mathrm{H}\right), 128.9$ $(\mathrm{m}, 8 \mathrm{C}, C \mathrm{H}), 129.2(\mathrm{~s}, 4 \mathrm{C}, C \mathrm{H}), 132.1(\mathrm{~s}, 4 \mathrm{C}, C \mathrm{H}), 133.2(\mathrm{~m}, 8 \mathrm{C}, C \mathrm{H}), 133.3\left(\mathrm{~d},{ }^{2} J_{\mathrm{CP}}=23.3 \mathrm{~Hz}\right.$, 8C, $C \mathrm{H}), 140.1(\mathrm{~m}, 8 \mathrm{C}, C \mathrm{P})$ ppm. FT-IR (ATR) v / $\mathrm{cm}^{-1}: 3049(\mathrm{w}), 2892(\mathrm{w}), 2571(\mathrm{w}), 2383$ (w), $1586(w), 1479$ (w), 1433 (w), 1402 (m), 1305 (w), 1261 (w), 1183 (w), 1158 (w), 1103 (m), 1069 (w), 1027 (w), 998 (s), 934 (m), 796 (m), 739 (s), 723 (s), 689 (s), 627 (m), 615 (m), $605(\mathrm{~m}), 542(\mathrm{w}), 516(\mathrm{~m}), 493(\mathrm{~s}), 465(\mathrm{~m}), 416(\mathrm{w}) . \operatorname{HRMS}\left(\mathrm{ESI}^{+}\right) \quad \mathrm{m} / \mathrm{z}=\quad 783.2551$ calcd. for $\left[\mathrm{C}_{48} \mathrm{H}_{44} \mathrm{BN}_{2} \mathrm{P}_{4}\right]^{+}$, found: $783.2554, \Delta=0.38 \mathrm{ppm}$. 
Synthesis of [ $\left.\mathbf{H}_{2} \mathbf{B}(\mathbf{d p p m})_{2}\right] \mathbf{B r}(\mathbf{1 b}) \operatorname{dppm}(880 \mathrm{mg}, 2.2 \mathrm{mmol})$ was dissolved in $\mathrm{CH}_{2} \mathrm{Cl}_{2}(6 \mathrm{~mL})$ and a solution of $\mathrm{BH}_{2} \mathrm{Br} \cdot \mathrm{SMe}_{2}$ in $\mathrm{CH}_{2} \mathrm{Cl}_{2}(1 \mathrm{M}, 1.1 \mathrm{~mL}, 1.1 \mathrm{mmoL})$ was added. After stirring for $1 \mathrm{~d}$, the ${ }^{31} \mathrm{P}\left\{{ }^{1} \mathrm{H}\right\}$-NMR spectrum of the reaction mixture indicated $\sim 80 \%$ conversion of $d p p m$ and the formation of $\sim 50 \% \mathbf{~ 1 b}$. Notably, continuous stirring of the reaction mixture (up to $10 \mathrm{~d}$ ) did not result in further conversion of $d p p m$. All volatiles were removed under reduced pressure. The residue was washed with $\mathrm{Et}_{2} \mathrm{O} / \mathrm{THF}(2: 1,5 \mathrm{x} 10 \mathrm{~mL})$ and dried under high vacuum to obtain $\left[\mathrm{H}_{2} \mathrm{~B}(\text { depa })_{2}\right] \mathrm{Br}(560 \mathrm{mg}, 0.65 \mathrm{mmol}, 59 \%)$ as colorless crystalline solid.

${ }^{1} \mathrm{H}-\mathrm{NMR}\left(300 \mathrm{MHz}, \mathrm{CD}_{2} \mathrm{Cl}_{2}\right) \delta=2.70\left(\mathrm{~d},{ }^{3} \mathrm{~J}_{\mathrm{HH}}=12.3 \mathrm{~Hz}, 4 \mathrm{H}, \mathrm{CH}_{2}\right), 7.23(\mathrm{~m}, 12 \mathrm{H}, \mathrm{CH}), 7.31$ $(\mathrm{m}, 16 \mathrm{H}, \mathrm{CH}), 7.38(\mathrm{~m}, 12 \mathrm{H}, \mathrm{CH})$ ppm. $\left(\mathrm{BH}_{3}\right.$-protons were not detected in $\mathrm{CD}_{2} \mathrm{Cl}_{2}$. Only resonances whose multiplicity is changing upon decoupling are reported in the ${ }^{1} \mathrm{H}\left\{{ }^{31} \mathrm{P}\right\}$ and ${ }^{1} \mathrm{H}\left\{{ }^{11} \mathrm{~B}\right\}$ NMR spectra. ${ }^{1} \mathrm{H}\left\{{ }^{11} \mathrm{~B}\right\}-\mathrm{NMR}\left(300 \mathrm{MHz}, \mathrm{CD}_{2} \mathrm{Cl}_{2}\right) \delta=2.80\left(\mathrm{t},{ }^{2} \mathrm{~J}_{\mathrm{HP}}=8.3 \mathrm{~Hz}, 2 \mathrm{H}, \mathrm{B} H_{2}\right)$ ppm. ${ }^{1} \mathrm{H}\left\{{ }^{31} \mathrm{P}\right\}-\mathrm{NMR}\left(300 \mathrm{MHz}, \mathrm{CD}_{2} \mathrm{Cl}_{2}\right) \delta=2.70\left(\mathrm{~s}, 2 \mathrm{H}, \mathrm{CH}_{2}\right)$ ppm. ${ }^{11} \mathrm{~B}\left\{{ }^{1} \mathrm{H}\right\}-\mathrm{NMR}(96 \mathrm{MHz}$, $\left.\mathrm{CD}_{2} \mathrm{Cl}_{2}\right) \delta=-34.0$ (br 1B) ppm. ${ }^{31} \mathrm{P}\left\{{ }^{1} \mathrm{H}\right\}-\mathrm{NMR}\left(122 \mathrm{MHz}, \mathrm{CD}_{2} \mathrm{Cl}_{2}\right) \delta=-28.8\left(\mathrm{~d},{ }^{3} J_{\mathrm{PP}}=69.9\right.$ $\mathrm{Hz}, 2 \mathrm{P}, \mathrm{BPCP}$ ), 7.64 (br, 2P, BPC) ppm. ${ }^{13} \mathrm{C}-\mathrm{NMR}\left(75 \mathrm{MHz}, \mathrm{CD}_{2} \mathrm{Cl}_{2}\right) \delta=22.3\left(2 \mathrm{C}, \mathrm{P}-\mathrm{CH}_{2}-\mathrm{P}\right)$, $124.3(\mathrm{~m}, 4 \mathrm{C}), 129.0\left(\mathrm{~d},{ }^{3} J_{\mathrm{CP}}=8.0 \mathrm{~Hz}, 8 \mathrm{C}\right), 129.7\left(\mathrm{~d},{ }^{3} J_{\mathrm{CP}}=11.3 \mathrm{~Hz}, 8 \mathrm{C}\right), 129.8$ (s, 4C), 132.9 $\left(\mathrm{d},{ }^{2} J_{\mathrm{CP}}=9.7 \mathrm{~Hz}, 8 \mathrm{C}\right), 133.1\left(\mathrm{~d},{ }^{2} J_{\mathrm{CP}}=22.0 \mathrm{~Hz}, 8 \mathrm{C}\right), 133.3\left(\mathrm{~d},{ }^{4} J_{\mathrm{CP}}=2.7 \mathrm{~Hz}, 4 \mathrm{C}\right), 136.4\left(\mathrm{dd},{ }^{1} J_{\mathrm{CP}}=\right.$ $\left.13.3 \mathrm{~Hz},{ }^{3} J_{\mathrm{CP}}=7.4 \mathrm{~Hz}, 4 \mathrm{C}\right) \mathrm{ppm}$. FT-IR (ATR) $\tilde{v} / \mathrm{cm}^{-1}: 3050(\mathrm{w}), 3007(\mathrm{w}), 2983(\mathrm{w}), 2846$ (w), $2440(\mathrm{w}), 2394$ (w), 1586 (w), 1482 (w), 1435 (m), 1365 (w), 1313 (w), 1189 (w), 1160 (w), 1106 (m), 1070 (w), 1026 (w) 997 (w), 915 (w), 776 (m), 740 (s), 721 (m), 690 (s), 633 (m), 584 (w), 542 (w), 505 (m), 492 (m), 465 (m), 430 (w). HRMS (ESI ${ }^{+}$m/z = 781.2646 calcd. for $\left[\mathrm{C}_{50} \mathrm{H}_{46} \mathrm{BP}_{4}\right]^{+}$, found: $781.2645, \Delta=-0.13 \mathrm{ppm}$.

Synthesis of $\left[\mathrm{H}_{2} \mathbf{B}(\mathbf{d e p a})_{2}\right] \mathrm{Br}$ (1c) depa $(80 \mathrm{mg}, 0.42 \mathrm{mmol})$ was dissolved in $\mathrm{CH}_{2} \mathrm{Cl}_{2}(5 \mathrm{~mL})$ and a solution of $\mathrm{BH}_{2} \mathrm{Br} \cdot \mathrm{SMe}_{2}$ in $\mathrm{CH}_{2} \mathrm{Cl}_{2}(1 \mathrm{M}, 0.21 \mathrm{~mL}, 0.21 \mathrm{mmol})$ was added via syringe. After stirring for $18 \mathrm{~h}$, the ${ }^{31} \mathrm{P}\left\{{ }^{1} \mathrm{H}\right\}$-NMR spectrum of the reaction mixture indicated full conversion of depa. The solvent was removed under reduced pressure and the waxy residue was washed with $n$-hexane $(3 \times 5 \mathrm{~mL})$. The solvent was removed under high vacuum to obtain $\left[\mathrm{H}_{2} \mathrm{~B}(\text { depa })_{2}\right] \mathrm{Br}(100 \mathrm{mg}, 0.20 \mathrm{mmol}, 98 \%)$ as a colorless oil.

${ }^{1} \mathrm{H}-\mathrm{NMR}\left(500 \mathrm{MHz}, \mathrm{C}_{6} \mathrm{D}_{6}\right) \delta=0.99\left(\mathrm{~m}, 12 \mathrm{H}, \mathrm{CH}_{3}\right), 1.14(\mathrm{~m}, 12 \mathrm{H}, \mathrm{CH}), 1.74\left(\mathrm{~m}, 4 \mathrm{H}, \mathrm{CH}_{2}\right)$, $1.82\left(\mathrm{~m}, 8 \mathrm{H}, \mathrm{CH}_{2}\right), 2.20$ (sex, $J=7.6 \mathrm{~Hz}, 4 \mathrm{H}, \mathrm{CH}_{2}$ ) ppm. (NH- and $\mathrm{BH}_{3}$-protons were not detected in $\mathrm{C}_{6} \mathrm{D}_{6}$ ). Only resonances whose multiplicity is changing upon decoupling are reported in the ${ }^{1} \mathrm{H}\left\{{ }^{31} \mathrm{P}\right\}$ and ${ }^{1} \mathrm{H}\left\{{ }^{11} \mathrm{~B}\right\}$ NMR spectra. ${ }^{1} \mathrm{H}\left\{{ }^{11} \mathrm{~B}\right\}-\mathrm{NMR}\left(500 \mathrm{MHz}, \mathrm{C}_{6} \mathrm{D}_{6}\right) \delta=1.46$ 
$\left(\mathrm{t},{ }^{2} J_{\mathrm{HP}}=19.6 \mathrm{~Hz}, 2 \mathrm{H}, \mathrm{B} H_{2}\right) \mathrm{ppm} .{ }^{1} \mathrm{H}\left\{{ }^{31} \mathrm{P}\right\}-\mathrm{NMR}\left(500 \mathrm{MHz}, \mathrm{C}_{6} \mathrm{D}_{6}\right) \delta=0.99\left(\mathrm{t},{ }^{3} J_{\mathrm{HH}}=7.6 \mathrm{~Hz}\right)$, $1.22\left(\mathrm{~m}, 18 \mathrm{H}, \mathrm{CH}_{3}\right), 1.74$ (sex, $\left.J=7.5 \mathrm{~Hz}, 4 \mathrm{H}, \mathrm{CH}_{2}\right), 1.82$ (sex, $J=7.6 \mathrm{~Hz}, 12 \mathrm{H}, \mathrm{CH}_{2}$ ) ppm. ${ }^{11} \mathrm{~B}\left\{{ }^{1} \mathrm{H}\right\}-\mathrm{NMR}\left(161 \mathrm{MHz}, \mathrm{C}_{6} \mathrm{D}_{6}\right) \quad \delta=-37.2$ (br t, $\left.{ }^{1} J_{\mathrm{BP}}=106.8 \mathrm{~Hz}\right)$ ppm. ${ }^{31} \mathrm{P}\left\{{ }^{1} \mathrm{H}\right\}-\mathrm{NMR}$ $\left(500 \mathrm{MHz}, \mathrm{C}_{6} \mathrm{D}_{6}\right) \delta=47.1$ (br, 2P, (B-P-N-P)2), 54,2 (br d, 2P, $P$-B-P) ppm. ${ }^{31} \mathrm{P}\left\{{ }^{1} \mathrm{H}\right\}-\mathrm{NMR}$ $\left(300 \mathrm{MHz}, \mathrm{CH}_{2} \mathrm{Cl}_{2}\right) \delta=17.2\left(\mathrm{~d},{ }^{2} J_{\mathrm{PP}}=18.2 \mathrm{~Hz}, 2 \mathrm{P},(\mathrm{B}-\mathrm{P}-\mathrm{N}-\mathrm{P})_{2}\right), 43\left(\mathrm{q},{ }^{1} J_{\mathrm{PB}}=105.0 \mathrm{~Hz}, 2 \mathrm{P}, P-\right.$ B-P) ppm. ${ }^{13} \mathrm{C}$-APT-NMR $\left(76 \mathrm{MHz}, \mathrm{C}_{6} \mathrm{D}_{6}\right) \delta=6.7\left(\mathrm{~d},{ }^{3 / 2} J_{\mathrm{CP}}=22.3 \mathrm{~Hz} 4 \mathrm{C}, C_{3}\right), 9.7\left(\mathrm{~d},{ }^{3 / 2} J_{\mathrm{CP}}=\right.$ $\left.13.8 \mathrm{~Hz}, 4 \mathrm{C}, \mathrm{CH}_{2}\right), 20.7\left(\mathrm{~d},{ }^{3 / 2} \mathrm{~J}_{\mathrm{CP}}=46.9 \mathrm{~Hz}, 4 \mathrm{C}, C \mathrm{H}_{2}\right), 24.3\left(\mathrm{~d},{ }^{3 / 2} \mathrm{~J}_{\mathrm{CP}}=10.5 \mathrm{~Hz}, 4 \mathrm{C}, C \mathrm{H}_{2}\right) \mathrm{ppm}$. FT-IR (ATR) v / cm ${ }^{-1}: 2967$ (m), 2934 (m), 2902 (m), 2877 (m), 2655 (m, 2378 (B-H, w), 1455.8 (m), 1407 (m), 1378 (m), 1219 (s), 1149 (m), 1033 (m), 1003 (m), 937 (s), 889 (w), $796(\mathrm{~m}), 764$ (s), 730 (m), 695 (m), 599 (w). HRMS $\left(\mathrm{ESI}^{+}\right) \mathrm{m} / z=399.2542$ calcd. for [C $\left.\mathrm{C}_{16} \mathrm{H}_{44} \mathrm{BN}_{2} \mathrm{P}_{4}\right]^{+}$; found: $399.2544, \Delta=0.50 \mathrm{ppm}$.

Synthesis of $\left[\left(\mathbf{B H}_{2}(\text { depa })_{2}\right) \mathbf{F e}(\mathbf{C O})_{3}\right] \mathbf{B r}(\mathbf{2 c})\left[\mathrm{H}_{2} \mathrm{~B}(\text { depa })_{2}\right] \mathrm{Br}(\mathbf{1 c}, 479 \mathrm{mg}, 1.0 \mathrm{mmol})$ was dissolved in $\mathrm{CH}_{2} \mathrm{Cl}_{2}(8 \mathrm{~mL})$ and $\left[\mathrm{Fe}(\mathrm{CO})_{5}\right](135 \mu \mathrm{L}, 1.0 \mathrm{mmol})$ was added. The solution was exposed to UV-irradiation for $2 \mathrm{~h}$. The extent of conversion was checked via ${ }^{31} \mathrm{P}\left\{{ }^{1} \mathrm{H}\right\} \mathrm{NMR}$ spectroscopy, showing $76 \%$ conversion of 1c. The mixture was filtered through a syringe filter and layered with $n$-hexane, resulting in single crystals suitable for X-ray-diffraction. The solvent was decanted, the residue was washed with $\mathrm{Et}_{2} \mathrm{O}(2 \times 5 \mathrm{~mL})$ and the orange crystals of 2d were dissolved in $\mathrm{CH}_{2} \mathrm{Cl}_{2}$. Removal of the solvent under reduced pressure resulted in $\left[\left(\mathrm{BH}_{2}(\mathrm{depa})_{2}\right) \mathrm{Fe}(\mathrm{CO})_{3}\right] \mathrm{Br}(23 \mathrm{mg}, 37 \mu \mathrm{mol}, 4 \%)$ as orange crystalline solid.

${ }^{1} \mathrm{H}-\mathrm{NMR}\left(300 \mathrm{MHz}, \mathrm{CDCl}_{3}\right) \delta=1.30-1.18\left(\mathrm{~m}, 24 \mathrm{H}, \mathrm{CH}_{3}\right), 2.11-1.94\left(\mathrm{~m}, 8 \mathrm{H}, \mathrm{CH}_{2}\right.$ ), 2.25 (sept, $\left.J=7.3 \mathrm{~Hz}, 4 \mathrm{H}, \mathrm{CH}_{2}\right), 2.51$ (sept, $J=7.5 \mathrm{~Hz}, 4 \mathrm{H}, \mathrm{CH}_{2}$ ), 5.83 (s, 2H, $\mathrm{NH}$ ) ppm. Only resonances whose multiplicity is changing upon decoupling are reported in the ${ }^{1} \mathrm{H}\left\{{ }^{31} \mathrm{P}\right\}$ and ${ }^{1} \mathrm{H}\left\{{ }^{11} \mathrm{~B}\right\} \mathrm{NMR}$ spectra. ${ }^{1} \mathrm{H}\left\{{ }^{31} \mathrm{P}\right\}-\mathrm{NMR}\left(300 \mathrm{MHz}, \mathrm{CDCl}_{3}\right) \delta=1.21\left(\mathrm{t},{ }^{3} \mathrm{~J}_{\mathrm{HH}}=6.9 \mathrm{~Hz}, 12 \mathrm{H}, \mathrm{CH}\right), 1.25\left(\mathrm{t},{ }^{3} J_{\mathrm{HH}}=\right.$ $\left.7.5 \mathrm{~Hz}, 12 \mathrm{H}, \mathrm{CH}_{3}\right), 2.06$ (quin, $J=6.7 \mathrm{~Hz}, \mathrm{ppm} .{ }^{1} \mathrm{H}\left\{{ }^{11} \mathrm{~B}\right\}-\mathrm{NMR}\left(300 \mathrm{MHz}, \mathrm{CDCl}_{3}\right) \delta=1.70(\mathrm{t}$, $\left.{ }^{2} J_{\mathrm{HP}}=16.3 \mathrm{~Hz}, 2 \mathrm{H}, \mathrm{BH} H_{2}\right) \mathrm{ppm} .{ }^{11} \mathrm{~B}\left\{{ }^{1} \mathrm{H}\right\}-\mathrm{NMR}\left(96 \mathrm{MHz}, \mathrm{CDCl}_{3}\right) \delta=-12.1\left(\mathrm{~m}, \mathrm{br}, 1 \mathrm{~B}, B \mathrm{H}_{2}\right)$ ppm. ${ }^{31} \mathrm{P}\left\{{ }^{1} \mathrm{H}\right\}-\mathrm{NMR}\left(122 \mathrm{MHz}, \mathrm{CDCl}_{3}\right) \delta=50.2$ (s, br, 2P, $P$-B-P), 124.5 (s, 2P, $P$-Fe- $P$ ) ppm. ${ }^{13} \mathrm{C}\left\{{ }^{1} \mathrm{H}\right\}$-NMR $\left(126 \mathrm{MHz}, \mathrm{CDCl}_{3}\right) \delta=6.90\left(\mathrm{~s}, 4 \mathrm{C}, \mathrm{CH}_{3}\right) 8.9\left(\mathrm{~s}, 4 \mathrm{C}, \mathrm{CH}_{3}\right), 21.8\left(\mathrm{vt},{ }^{1} \mathrm{~J}_{\mathrm{CP}}=\right.$ $26.1 \mathrm{~Hz}, 4 \mathrm{C}, \mathrm{CH}_{2}$ ), 30.6 (vt, ${ }^{1} J_{\mathrm{CP}}=16.2 \mathrm{~Hz}, 4 \mathrm{C}, 216.0$ (m br, 3C, $\mathrm{CO}$ ), CH2) ppm. FT-IR (ATR) $\tilde{v} / \mathrm{cm}^{-1}: 2972$ (w), 2937 (w), 2705 (w), 1972 (s, CO), 1890 (s, CO), 1866 (s, CO), 1455 (m), 1407 (m), 1379 (m), 1243 (m), 1037 (m), 933 (s), 791 (s), 769 (s), 736 (s), 641 (s), 589 (s), 551 (w), $510(\mathrm{w}), 443(\mathrm{w})$. HRMS $\left(\mathrm{ESI}^{+}\right) \mathrm{m} / \mathrm{z}=539.1743$ calcd. for $\left[\mathrm{C}_{18} \mathrm{H}_{44} \mathrm{BFeN}_{2} \mathrm{O}_{2} \mathrm{P}_{4}\right]^{+}$, found: $539.1739, \Delta=-0.74 \mathrm{ppm}$. 
Continued irradiation of the combined $\mathrm{Et}_{2} \mathrm{O}$-layers resulted in the formation of $\left[\left(\mathrm{BH}(\mathrm{depa})_{2}\right) \mathrm{Fe}(\mathrm{H})(\mathrm{CO})_{2}\right] \mathrm{Br}(\mathbf{3 c})$. The solvent was removed under reduced pressure and the red residue was washed with toluene $(2 \times 5 \mathrm{~mL})$ and precipitated from THF $(1.5 \mathrm{~mL})$ with toluene $(10 \mathrm{~mL})$. The residue was separated and dried under high vacuum to obtain $\left[\left(\mathrm{BH}(\mathrm{depa})_{2}\right) \mathrm{Fe}(\mathrm{H})(\mathrm{CO})_{2}\right] \mathrm{Br}(59 \mathrm{mg}, 0.1 \mathrm{mmol}, 10 \%)$ as orange crystalline solid.

\section{Synthesis of $\left[\left(\mathrm{BH}(\mathrm{dppa})_{2}\right) \mathrm{Fe}(\mathrm{H})(\mathrm{CO})_{2}\right] \mathrm{Br}(3 a)$}

$48 \mathrm{mg}\left[\mathrm{H}_{2} \mathrm{~B}(\text { dppa })_{2}\right] \mathrm{Br}(55.6 \mu \mathrm{mol})$ was dissolved in THF $(10 \mathrm{~mL})$ and $\mathrm{Fe}(\mathrm{CO})_{5}(102 \mu \mathrm{mol}$, 1.8eq) was added. The reaction mixture was irradiated with UV- light for $40 \mathrm{~min}$, while stirring and cooling with a water bath. The resulting orange suspension was filtered and the filtrate was added dropwise to hexane $(35 \mathrm{~mL})$, to precipitate 3a. The supernatant was removed and the residue washed with $\mathrm{Et}_{2} \mathrm{O}(10 \mathrm{~mL})$. After drying in high vacuum $45 \mathrm{mg}$ of $\mathbf{3 a}$ were obtained as pale crystalline solid in $50 \%$ yield $(27.1 \mathrm{mg}, 27.8 \mu \mathrm{mol}, 50 \%)$. The analytical data is in agreement with previously published data. ${ }^{3}$

Synthesis of $\left[\left(\mathbf{B H}(\mathbf{d p p m})_{2}\right) \mathbf{F e}(\mathbf{H})(\mathbf{C O})_{2}\right] \mathbf{B r}(\mathbf{3 b})\left[\mathrm{H}_{2} \mathrm{~B}(\mathrm{dppm})_{2}\right] \mathrm{Br}(\mathbf{1 b}, 250 \mathrm{mg}, 290 \mu \mathrm{mol})$ was dissolved in $\mathrm{CH}_{2} \mathrm{Cl}_{2}(5 \mathrm{~mL})$. [ $\left.\mathrm{Fe}(\mathrm{CO})_{5}\right](0.1 \mathrm{~mL}, 740 \mu \mathrm{mol})$ was added and the reaction was irradiated with UV-light for $2 \mathrm{~h}$. The ${ }^{31} \mathrm{P}\left\{{ }^{1} \mathrm{H}\right\}$ NMR spectrum showed full conversion to $\mathbf{3 b}$. The solvent was evaporated under reduced pressure, the residue washed with toluene $(2 \times 7 \mathrm{~mL})$ and $\mathrm{Et}_{2} \mathrm{O}(2 \times 8 \mathrm{~mL})$ and precipitated from $\mathrm{CH}_{2} \mathrm{Cl}_{2}(1 \mathrm{~mL})$ with $\mathrm{Et}_{2} \mathrm{O}(30 \mathrm{~mL})$. The remaining solid was washed with $\mathrm{Et}_{2} \mathrm{O}(20 \mathrm{~mL}, 10 \mathrm{~mL})$ until the $\mathrm{Et}_{2} \mathrm{O}$ - layers remained colorless. Drying the residue under high vacuum resulted in $\left[\left(\mathrm{BH}(\mathrm{dppm})_{2}\right) \mathrm{Fe}(\mathrm{H})(\mathrm{CO})_{2}\right] \mathrm{Br} \mathbf{3 b}(146 \mathrm{mg}, 147 \mu \mathrm{mol}$, $51 \%)$ as a pale solid.

${ }^{1} \mathrm{H}-\mathrm{NMR}\left(500 \mathrm{MHz}, \mathrm{CD}_{3} \mathrm{CN}\right) \delta=3.84\left(\mathrm{br}, 4 \mathrm{H}, \mathrm{CH}_{2}\right), 7.18-7.26\left(\mathrm{~m}, 8 \mathrm{H}, H^{P h}\right), 7.26-7.42(\mathrm{~m}$, $\left.24 \mathrm{H}, H^{P h}\right), 7.42-7.49\left(\mathrm{~m}, 8 \mathrm{H}, H^{P h}\right) \mathrm{ppm}$. Only resonances whose multiplicity is changing upon decoupling or cooling are reported in the ${ }^{1} \mathrm{H}\left\{{ }^{31} \mathrm{P}\right\}$ and ${ }^{1} \mathrm{H}\left\{{ }^{11} \mathrm{~B}\right\}$ NMR spectra. ${ }^{1} \mathrm{H}-\mathrm{NMR}$ $\left(500 \mathrm{MHz}, \mathrm{CD}_{3} \mathrm{CN},-33^{\circ} \mathrm{C}\right) \delta=-9.50$ (br t, $1 \mathrm{H}, \mathrm{Fe} H$ ), 3.43 (br, $1 \mathrm{H}, \mathrm{BH}$ ), 3.84 (br d, $J=240 \mathrm{~Hz}$, $\left.4 \mathrm{H}, \mathrm{CH}{ }_{2}\right)$ ppm. ${ }^{1} \mathrm{H}\left\{{ }^{31} \mathrm{P}\right\}-\mathrm{NMR}\left(500 \mathrm{MHz}, \mathrm{CD}_{3} \mathrm{CN},-33^{\circ} \mathrm{C}\right) \delta=-9.50$ (br s, $1 \mathrm{H}, \mathrm{Fe} H$ ) ppm. ${ }^{1} \mathrm{H}\left\{{ }^{11} \mathrm{~B}\right\}-\mathrm{NMR}\left(500 \mathrm{MHz}, \mathrm{CD}_{3} \mathrm{CN},-33^{\circ} \mathrm{C}\right) \delta=-9.51$ (br t, $\left.1 \mathrm{H}, \mathrm{Fe} H\right), 3.23$ (br, $\left.1 \mathrm{H}, \mathrm{BH}\right), 3.84$ (br d, $\left.J=240 \mathrm{~Hz}, 4 \mathrm{H}, \mathrm{CH}_{2}\right) \mathrm{ppm} .{ }^{1} \mathrm{H}\left\{{ }^{31} \mathrm{P}\right\}-\mathrm{NMR}\left(300 \mathrm{MHz}, \mathrm{CD}_{3} \mathrm{CN}\right) \delta=3.89$ (br d, $J=8.8 \mathrm{~Hz}$, $\left.4 \mathrm{H}, \mathrm{CH}_{2}\right) \mathrm{ppm} .{ }^{11} \mathrm{~B}\left\{{ }^{1} \mathrm{H}\right\}-\mathrm{NMR}\left(96 \mathrm{MHz}, \mathrm{CD}_{3} \mathrm{CN}\right) \delta=-23.0 \mathrm{ppm} .{ }^{11} \mathrm{~B}\left\{{ }^{1} \mathrm{H}\right\}-\mathrm{NMR}(160 \mathrm{MHz}$, $\left.\mathrm{CD}_{2} \mathrm{Cl}_{2}\right) \delta=-23.2$ ppm. ${ }^{31} \mathrm{P}\left\{{ }^{1} \mathrm{H}\right\}-\mathrm{NMR}\left(202 \mathrm{MHz}, \mathrm{CD}_{3} \mathrm{CN}\right) \delta=21.9$ (br, 2P, $P$-B-P), $77.6(\mathrm{dd}$, $J=47.5 ; 66.6 \mathrm{~Hz}, 2 \mathrm{P}, P$-Fe- $P$ ) ppm. ${ }^{31} \mathrm{P}\left\{{ }^{1} \mathrm{H}\right\}-\mathrm{NMR}\left(202 \mathrm{MHz}, \mathrm{CD}_{3} \mathrm{CN},-33^{\circ} \mathrm{C}\right) \delta=22.2(\mathrm{br}$, 
2P, $P$-B-P), 78.2 (dd, $J=47.1 ; \quad 66.7 \mathrm{~Hz}, 2 \mathrm{P}, \quad P$-Fe- $P$ ) ppm. ${ }^{31} \mathrm{P}\left\{{ }^{1} \mathrm{H}\right\}-\mathrm{NMR} \quad(122 \mathrm{MHz}$, $\left.\mathrm{CD}_{2} \mathrm{Cl}_{2}\right) \delta=21.5$ (br, 2P, $P$-B- $P$ ), 77.9 (dd, $J=47.4 ; 65.6 \mathrm{~Hz}, 2 \mathrm{P}, P-\mathrm{Fe}-P$ ) ppm. ${ }^{13} \mathrm{C}-\mathrm{NMR}$ $\left(126 \mathrm{Mhz}, \mathrm{CD}_{3} \mathrm{CN}\right): \delta=38.9(\mathrm{~m}, 2 \mathrm{C}, \mathrm{PCP}), 125.3\left(\mathrm{~d},{ }^{1} J_{P C}=61.8 \mathrm{~Hz}, 4 \mathrm{C}^{\text {quart }}\right), 128.6\left(\mathrm{t},{ }^{2} J_{P C}=\right.$ $4.9 \mathrm{~Hz}, 8 \mathrm{C}), 129.4\left(\mathrm{t},{ }^{2} J_{P C}=5.0 \mathrm{~Hz}, 8 \mathrm{C}\right), 130.6(\mathrm{~s}, 2 \mathrm{C}), 132.2\left(\mathrm{t},{ }^{3} J_{P C}=5.7 \mathrm{~Hz}, 8 \mathrm{C}\right), 132.4(\mathrm{~s}$, 2C), $132.8\left(\mathrm{t},{ }^{3} J_{P C}=4.8 \mathrm{~Hz}, 8 \mathrm{C}\right), 136.5\left(\mathrm{~m}, 4 \mathrm{C}^{\text {quart }}\right), 215.1(\mathrm{br}, 2 \mathrm{C}, \mathrm{CO}) \mathrm{ppm}$. FT-IR (ATR) v / $\mathrm{cm}^{-1}$ : 3054 (vw), 3021 (vw), 2928 (vw), 2855 (vw), 2356 (vw), 1973 (s), 1927 (s), $1896(\mathrm{~m})$, 1587 (vw), 1573 (vw), 1484 (m), 1434 (s), 1373 (w), 1334 (vw), 1306 (w), $1276(\mathrm{w}), 1188(\mathrm{w})$, 1160 (w), 1136 (w), 1093 (m), 1026 (w), 998 (m), 823 (w), 792 (m), 760 (m), 723 (vs), 688 (vs), 608 (s), 587 (m), $566(\mathrm{~m}), 557$ (m), 525 (m), 501 (s), 483 (s), 470 (s), 459 (m), 429 (m), $407(\mathrm{w})$. HRMS $\left(\mathrm{ESI}^{+}\right) \mathrm{m} / \mathrm{z}=893.1895$ calculated for $\left[\mathrm{C}_{52} \mathrm{H}_{46} \mathrm{BFeO}_{2} \mathrm{P}_{4}\right]^{+}$, found: $893.1914, \Delta=$ $2.13 \mathrm{ppm}$. Elemental analysis (measured [calculated] for $\mathrm{C}_{53} \mathrm{H}_{48} \mathrm{BBrCl}_{2} \mathrm{FeO}_{2} \mathrm{P}_{4}$ ): 60.36 [60.15] C, $4.58[4.57] \mathrm{H}, 0.00[0.00] \mathrm{N}$.

Synthesis of $\left[\left(\mathbf{B H}(\mathbf{d e p a})_{2}\right) \mathbf{F e}(\mathbf{H})(\mathbf{C O})_{2}\right] \mathbf{B r}(\mathbf{3 c})$ depa $(572 \mathrm{mg}, 3.0 \mathrm{mmol})$ was dissolved in $\mathrm{CH}_{2} \mathrm{Cl}_{2}(20 \mathrm{~mL})$ and a solution of $\mathrm{BH}_{2} \mathrm{Br} \cdot \mathrm{SMe}_{2}$ in $\mathrm{CH}_{2} \mathrm{Cl}_{2}(1 \mathrm{M}, 1.5 \mathrm{~mL}, 1.5 \mathrm{mmol})$ was added. After stirring for $18 \mathrm{~h}$, reaction control via ${ }^{31} \mathrm{P}\left\{{ }^{1} \mathrm{H}\right\}$-NMR indicated full conversion of depa and formation of $\left[\mathrm{BH}_{2}(\text { depa })_{2}\right] \mathrm{Br}$. The solvent was removed under reduced pressure, the residue was dissolved in THF $(15 \mathrm{~mL})$ and $\left[\mathrm{Fe}(\mathrm{CO})_{5}\right](0.2 \mathrm{~mL}, 2.7 \mathrm{mmol})$ was added. After exposing the reaction to UV-irradiation for $1.5 \mathrm{~h}$, formation of orange and colorless crystals occurred. The supernatant was discarded and the residue was washed with toluene $(10 \mathrm{~mL})$ and suspended in $\mathrm{CH}_{2} \mathrm{Cl}_{2} / \mathrm{THF}$ ( $3 \mathrm{~mL}: 10 \mathrm{~mL}$ ) and exposed to UV-irradiation for $2.5 \mathrm{~h}$. The solvent was removed under reduced pressure and the residue was dissolved in $\mathrm{CHCl}_{3} .{ }^{31} \mathrm{P}\left\{{ }^{1} \mathrm{H}\right\} \mathrm{NMR}$ spectroscopy indicated full conversion to $\left[\left(\mathrm{BH}(\mathrm{depa})_{2}\right) \mathrm{Fe}(\mathrm{H})(\mathrm{CO})_{2}\right] \mathrm{Br}(\mathbf{3 c})$, which was precipitated with toluene $(20 \mathrm{~mL})$. The solvent was removed under high vacuum to obtain $\left[\left(\mathrm{BH}(\mathrm{depa})_{2}\right) \mathrm{Fe}(\mathrm{H})(\mathrm{CO})_{2}\right] \mathrm{Br}(124 \mathrm{mg}, 0.21 \mathrm{mmol}, 14 \%)$ as a pale yellow solid.

${ }^{1} \mathrm{H}-\mathrm{NMR}\left(500 \mathrm{MHz}, \mathrm{CD}_{2} \mathrm{Cl}_{2}\right.$ ) $\delta=-10.77$ (br, 1H, FeH) -9.90 (br, 1H, FeH), 1.15 (s, br, $24 \mathrm{H}$, $\mathrm{CH}_{3}$ ), 2.65-1.64 (m br, $18 \mathrm{H}, \mathrm{CH}_{2}$ ), 5.31 (br, 2H, NH) ppm. ${ }^{1} \mathrm{H}-\mathrm{NMR}\left(500 \mathrm{MHz}, \mathrm{CD}_{2} \mathrm{Cl}_{2},-60\right.$ $\left.{ }^{\circ} \mathrm{C}\right) \delta=-10.9\left(\mathrm{t},{ }^{2} J_{\mathrm{HP}}=53.5 \mathrm{~Hz}, 1 \mathrm{H}, \mathrm{Fe} H\right),-10.0\left(\mathrm{t},{ }^{2} J_{\mathrm{HP}}=60.0 \mathrm{~Hz}, 1 \mathrm{H}, \mathrm{Fe} H\right)^{c i s}, 0.83-1.24(\mathrm{~s}$, br, $24 \mathrm{H}, \mathrm{CH}_{3}$ ), 1.24-1.60 (m br, $4 \mathrm{H}, \mathrm{CH}_{2}$ ), 1.60-1.83(m br, $4 \mathrm{H}, \mathrm{CH}_{2}$ ), 1.83- 2.42 (m br, $8 \mathrm{H}$, $\mathrm{CH}_{2}$ ), 5.28 (br, 2H, NH) ppm. ${ }^{1} \mathrm{H}\left\{{ }^{31} \mathrm{P}\right\}-\mathrm{NMR}\left(500 \mathrm{MHz}, \mathrm{CD}_{2} \mathrm{Cl}_{2},-60{ }^{\circ} \mathrm{C}\right) \delta=-10.9(\mathrm{~s}, 1 \mathrm{H}$, $\mathrm{Fe} H),-10.0(\mathrm{~s}, 1 \mathrm{H}, \mathrm{Fe} H){ }^{c i s} \mathrm{ppm} .{ }^{11} \mathrm{~B}\left\{{ }^{1} \mathrm{H}\right\}-\mathrm{NMR}\left(161 \mathrm{MHz}, \mathrm{CD}_{2} \mathrm{Cl}_{2},-60{ }^{\circ} \mathrm{C}\right) \delta=-30.7\left(\mathrm{t},{ }^{1} J_{\mathrm{BP}}=\right.$ 115.4 Hz, 1B, P-B-P) ppm. ${ }^{31} \mathrm{P}\left\{{ }^{1} \mathrm{H}\right\}-\mathrm{NMR}\left(122 \mathrm{MHz}, \mathrm{CD}_{2} \mathrm{Cl}_{2}\right) \delta=65.9$ (s br, 1B, $P$-B-P), 138.4 
$\left(\mathrm{dd},{ }^{2} J_{\mathrm{PP}}=57.6 \mathrm{~Hz},{ }^{2} J_{\mathrm{PP}}=48.3 \mathrm{~Hz}, 2 \mathrm{P}, P-\mathrm{Fe}-P\right), 139.8\left(\mathrm{dd},{ }^{2} J_{\mathrm{PP}}=54.5 \mathrm{~Hz},{ }^{2} J_{\mathrm{PP}}=66.9 \mathrm{~Hz}, 2 \mathrm{P}\right.$, $P$-Fe- $P)^{c i s}$, ppm. ${ }^{13} \mathrm{C}\left\{{ }^{1} \mathrm{H}\right\}-\mathrm{NMR}\left(125 \mathrm{MHz}, \mathrm{CD}_{2} \mathrm{Cl}_{2},-60{ }^{\circ} \mathrm{C}\right) \delta=7.4\left(\mathrm{~m}, 4 \mathrm{C}, C \mathrm{H}_{3}\right), 8.5(\mathrm{~s}, 2 \mathrm{C}$, $\left.\mathrm{CH}_{3}\right), 8.6\left(\mathrm{~m}, 4 \mathrm{C}, \mathrm{CH}_{3}\right), 19.1$ (m, 4C, $\left.\mathrm{CH}_{2}\right), 27.3$ (m, 2C, $\left.\mathrm{CH}_{2}\right), 31.7$ (m, 2C, $\mathrm{CH}_{2}$ ), 213.6 (s br, 1C, CO), 218.2 (s br, 1C, CO) ppm. FT-IR (ATR) ṽ / cm-1: 2968 (m), 2595 (w), 2382 (w, BH), 1958 (s, CO), 1912 (s, CO), 1876 (m), 1254 (m), 1411 (w), 1280 (w), 1304 (m), 1259 (w), 1037 (m), 924 (s), 821 (s), 753 (s), 699 (m), 659 (s), 611 (s), 576 (w), 526 (m), 485 (m), 449 (m). HRMS $\left(\mathrm{ESI}^{+}\right) \mathrm{m} / \mathrm{z}=511.1794$ calculated. for $\left[\mathrm{C}_{18} \mathrm{H}_{44} \mathrm{BFeN}_{2} \mathrm{O}_{2} \mathrm{P}_{4}\right]^{+}$found: $511.1791, \Delta=$ -0.59 ppm. Elemental analysis (measured [calculated] for $\mathrm{C}_{18} \mathrm{H}_{44} \mathrm{BBrFeN}_{2} \mathrm{O}_{2} \mathrm{P}_{4} / \%$ ): 35.59 [36.58] C, $7.21[7.50] \mathrm{H}, 5.04[4.74] \mathrm{N}$.

Spectroscopic data for complex $\mathbf{5 b}$ Complex $\mathbf{5 b}$ is selectively formed by the reaction of $\mathbf{3 b}$ with one equivalent $\mathrm{KO}^{t} \mathrm{Bu}$ in $\mathrm{C}_{6} \mathrm{D}_{6}$ or toluene- $\mathrm{d}_{8}$, but is slowly converted to complex $\mathbf{6 b}$. All attempts to isolate $\mathbf{5 b}$ as pure solid or to obtain suitable single crystals for X-Ray diffraction experiments failed. ${ }^{31} \mathrm{P}\left\{{ }^{1} \mathrm{H}\right\}$ NMR (202 MHz, C6 6 6, 298 K) $\delta: 17.8$ (br, P-B-P), 19.9 (br, P-B$P), 72.6\left(\mathrm{dd}, J_{\mathrm{PP}}=175.7 \mathrm{~Hz}, J_{\mathrm{PP}}=96.7 \mathrm{~Hz}, P-\mathrm{Fe}-\mathrm{P}\right), 98.9\left(\mathrm{td}, J_{\mathrm{PP}}=89.9 \mathrm{~Hz}, J_{\mathrm{PP}}=35.4 \mathrm{~Hz}, \mathrm{P}-\right.$ $\mathrm{Fe}-P$ ) ppm. ${ }^{11} \mathrm{~B}\left\{{ }^{1} \mathrm{H}\right\}$ NMR (160 MHz, $\left.\mathrm{C}_{6} \mathrm{D}_{6}, 298 \mathrm{~K}\right) \delta:-19.4$ (br, P-B-P) ppm. ${ }^{1} \mathrm{H}$ NMR (500 $\mathrm{MHz}, \mathrm{C}_{6} \mathrm{D}_{6}, 298 \mathrm{~K}$ ) $\delta:-8.67$ (br t, 1H, JPH = 57.2 Hz, Fe-H), 2.53 (br, 1H, P-CH-P), 3.01 (br, 1H P-CHH-P), 3.32 (br, 1H, P-CHH-P), 6.37-7.53 (superimposed br, 30H, phenyl-H), 7.70 (br, $2 \mathrm{H}$, phenyl- $H$ ), 7.87 (br, 4H, phenyl- $H$ ), 8.05 (br, $2 \mathrm{H}$, phenyl- $H$ ), 8.26 (br, $2 \mathrm{H}$, phenyl- $H$ ) ppm. Only resonances which are changing upon ${ }^{11} \mathrm{~B}$-decoupling are reported. ${ }^{1} \mathrm{H}\left\{{ }^{11} \mathrm{~B}\right\}$ NMR (500 $\mathrm{MHz}, \mathrm{C}_{6} \mathrm{D}_{6}, 298 \mathrm{~K}$ ) $\delta: 3.20$ (superimposed br, $1 \mathrm{H}, \mathrm{BH}$ ) ppm. ${ }^{31} \mathrm{P}$-decoupling of the boron-bound phosphorus atoms results in a sharpening of the triplet resonance observed for the hydrido ligand. ${ }^{1} \mathrm{H}\left\{{ }^{31} \mathrm{P}\right\}$ NMR $\left(500 \mathrm{MHz}, \mathrm{C}_{6} \mathrm{D}_{6}, 298 \mathrm{~K}, \mathrm{o} 2 \mathrm{p}=20 \mathrm{ppm}\right) \delta:-8.69$ (br t, $1 \mathrm{H},{ }^{2} J_{\mathrm{PH}}=50.6$ $\mathrm{Hz}, \mathrm{Fe} H$ ) ppm. ${ }^{31} \mathrm{P}$-decoupling of the iron-bound phosphorus atoms results in a broad doublet resonance for the hydrido ligand. ${ }^{1} \mathrm{H}\left\{{ }^{31} \mathrm{P}\right\} \mathrm{NMR}\left(500 \mathrm{MHz}, \mathrm{C}_{6} \mathrm{D}_{6}, 298 \mathrm{~K}, \mathrm{o} 2 \mathrm{p}=73 \mathrm{ppm}\right) \delta$ : 8.68 (br t, $\left.1 \mathrm{H}, J_{\mathrm{PH}}=45.1 \mathrm{~Hz}, \mathrm{Fe} H\right) \mathrm{ppm} .{ }^{13} \mathrm{C}\left\{{ }^{1} \mathrm{H}\right\} \mathrm{NMR}\left(126 \mathrm{MHz}, \mathrm{C}_{6} \mathrm{D}_{6}, 298 \mathrm{~K}\right) \mathrm{d}: 29.1$ (dd, $\left.\mathrm{J}_{\mathrm{PC}}=104.0 \mathrm{~Hz}, \mathrm{JPC}=60.3 \mathrm{~Hz}, \mathrm{P}-\mathrm{CH}-\mathrm{P}\right), 41.2\left(\mathrm{ddd}, J_{\mathrm{PC}}=55.1 \mathrm{~Hz}, J_{\mathrm{PC}}=16.6 \mathrm{~Hz}, \mathrm{JPC}=13.5\right.$ Hz, P-CH2-P), 124.7 /br, phenyl-C), 125.1 (br, phenyl-C), 127.6 (d, JPC = 9.4 Hz, phenyl-C), 131.1 (br, phenyl-C), 131.4 (br, phenyl-C), 131.9 (br, phenyl-C), 132.4 (d, JPC = $11.4 \mathrm{~Hz}$, phenyl-C), 133.0 (br, phenyl-C), 136.6 (br, phenyl-C), 139.8 (br, phenyl-C), 140.0 (br, phenylC), 141.0 (br, phenyl-C), 141.3 (br, phenyl-C), 142.0 (br, phenyl-C), 147.3 (br, phenyl-C), 147.6 (br, phenyl-C), 149.8 (br, phenyl-C), 216.3 (br, Fe-CO), 218.8 (br, Fe-CO) ppm. FT-IR (ATR) $\tilde{v} / \mathrm{cm}^{-1}: 1910(\mathrm{~s}), 1953(\mathrm{~m}) \mathrm{cm}^{-1}$.

\section{Spectroscopic data for complex $6 \mathrm{~b}$}


${ }^{31} \mathrm{P}\left\{{ }^{1} \mathrm{H}\right\}$ NMR $\left(122 \mathrm{MHz}, \mathrm{C}_{6} \mathrm{D}_{6}, 298 \mathrm{~K}\right) \delta: 26.1(\mathrm{br}, P-\mathrm{B}-P), 75.8\left(\mathrm{dt}, J_{\mathrm{PP}}=29.9 \mathrm{~Hz}, J_{\mathrm{PP}}=7.5\right.$ $\mathrm{Hz}, P-\mathrm{Fe}-P$ ) ppm. ${ }^{11} \mathrm{~B}\left\{{ }^{1} \mathrm{H}\right\} \mathrm{NMR}\left(96 \mathrm{MHz}, \mathrm{C}_{6} \mathrm{D}_{6}, 298 \mathrm{~K}\right) \delta:-37.2$ (br, P-B-P) ppm. ${ }^{1} \mathrm{H}$ NMR $\left(500 \mathrm{MHz}, \mathrm{C}_{6} \mathrm{D}_{6}, 298 \mathrm{~K}\right) \delta: 2.82$ (br, 4H, P-CH2-P), 6.4-8.6 (superimposed, phenyl- $H$ ) ppm. It was not possible to unambiguously assign the resonances of the carbon atoms of the phenyl rings, as $\mathbf{6 b}$ is always observed in a mixture with $\mathbf{5 b}, \mathbf{6} \mathbf{b}^{\mathbf{\prime}}$ or $\mathbf{7 b}$. Furthermore, we didn't detect the resonances of the carbonyl ligands. ${ }^{13} \mathrm{C}$ APT NMR $\left(126 \mathrm{MHz}, \mathrm{C}_{6} \mathrm{D}_{6}, 298 \mathrm{~K}\right) \delta: 32.1$ (br m, P-CH $2-\mathrm{P}$ ), 120-160 (superimposed, phenyl-C), 221.1 (br m, $\left.\mathrm{Fe}(C O)_{2}\right)$ ppm. FT-IR (ATR) v / $\mathrm{cm}^{-1}: 1833(\mathrm{~s}), 1881(\mathrm{~m}) \mathrm{cm}^{-1}$.

\section{Spectroscopic data for complex $6 b^{\prime}$}

${ }^{31} \mathrm{P}\left\{{ }^{1} \mathrm{H}\right\}$ NMR $\left(122 \mathrm{MHz}, \mathrm{C}_{6} \mathrm{D}_{6}, 298 \mathrm{~K}\right) \delta: 25.8(\mathrm{br}, P-\mathrm{B}-P), 79.8\left(\mathrm{dt}, J_{\mathrm{PP}}=29.9 \mathrm{~Hz}, J_{\mathrm{PP}}=6.7\right.$ $\mathrm{Hz}, P$-Fe- $P$ ) ppm. ${ }^{11} \mathrm{~B}\left\{{ }^{1} \mathrm{H}\right\}$ NMR $\left(96 \mathrm{MHz}, \mathrm{C}_{6} \mathrm{D}_{6}, 298 \mathrm{~K}\right) \delta:-37.2$ (br, P-B-P) ppm. ${ }^{1} \mathrm{H}$ NMR $(500 \mathrm{MHz}$, toluene-d $8,298 \mathrm{~K}) \delta: 2.96\left(\mathrm{dd}, 4 \mathrm{H},{ }^{2} J_{\mathrm{PH}}=11.1 \mathrm{~Hz},{ }^{2} J_{\mathrm{PH}}=5.2 \mathrm{~Hz}, \mathrm{P}-\mathrm{CH}_{2}-\mathrm{P}\right), 6.75-$ $7.06+7.29-7.51$ (superimposed, 30H, phenyl- $H$ ), 7.83 (m, 4H, phenyl- $H$ ), 7.99 (m, 6H, phenyl$H)$ ppm. Only resonances which are changing upon ${ }^{11} \mathrm{~B}$-decoupling are reported. ${ }^{1} \mathrm{H}\left\{{ }^{11} \mathrm{~B}\right\} \mathrm{NMR}$ (500 MHz, toluene-d8, $298 \mathrm{~K}) \delta: 4.02$ (br, BH) ppm.

\section{Spectroscopic data for complex $7 \mathrm{~b}$}

${ }^{31} \mathrm{P}\left\{{ }^{1} \mathrm{H}\right\}$ NMR $(202 \mathrm{MHz}$, tolene-d8, $298 \mathrm{~K}) \delta: 18.2$ (br, $P$-B-P), 60.4 (m, $P$-Fe- $P$ ) ppm. ${ }^{1} \mathrm{H}$ NMR (500 MHz, tolene-d8, 298 K) $\delta:-1.10$ (br, 1H, BH), 2.24 (br, 2H, P-CH-P) ppm. The resonances of the corresponding to the phenyl rings in $\mathbf{7 b}$ are superimposed with those of $\mathbf{5 b}$. Only resonances which are changing upon ${ }^{11} \mathrm{~B}$-decoupling are reported. ${ }^{1} \mathrm{H}\left\{{ }^{11} \mathrm{~B}\right\}$ NMR (500 $\left.\mathrm{MHz}, \mathrm{C}_{6} \mathrm{D}_{6}, 298 \mathrm{~K}\right) \delta$ : -1.11 (br, 1H, BH), 0.34 (br, 1H, BH) ppm. ${ }^{11} \mathrm{~B}\left\{{ }^{1} \mathrm{H}\right\}$ NMR $(160 \mathrm{MHz}$, tolene-d $8,298 \mathrm{~K}) \delta$ : -50.7 (br, P-B-P) ppm. It was not possible to unambiguously assign the resonances of the carbon atoms of the phenyl rings, as $\mathbf{7 b}$ is observed in a mixture with $\mathbf{5 b}$. Furthermore, we didn't detect the resonances of the carbonyl ligands. ${ }^{13}$ C-APT NMR (126 MHz, tolene-d 8,298 K) $\delta: 25.7$ (t, $\left.J_{\mathrm{PC}}=50 \mathrm{~Hz}, \mathrm{P}-\mathrm{CH}-\mathrm{P}\right) \mathrm{ppm}$. FT-IR (ATR) $\tilde{v} / \mathrm{cm}^{-1}: 1932$ (m), $1976(\mathrm{~m}) \mathrm{cm}^{-1}$. 


\section{Typical procedure for the dehydrogenation of dimethylaminoborane}

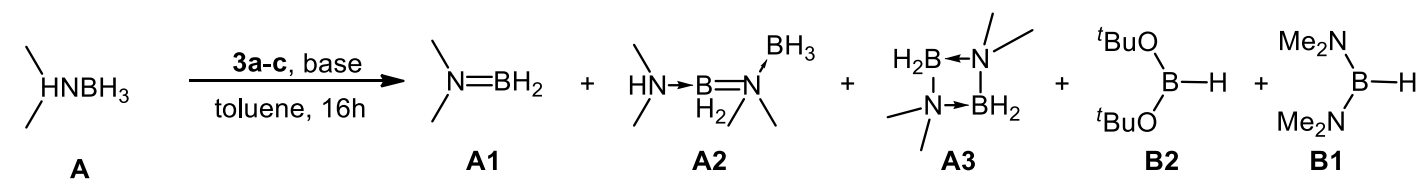

$\mathrm{c}\left(\mathrm{A}_{\text {ini }}\right)=0.27 \mathrm{~mol} / \mathrm{L}$

The complex 3a-c and the base were suspended in the corresponding solvent and stirred for 15 min, until a bright orange solution of the catalyst was formed. The solution was aliquoted $(1 \mathrm{~mL})$. One aliquot $(1 \mathrm{~mL})$ of a freshly prepared stock-solution of dimethylaminoborane $(0.54$ $\mathrm{mmoL}, 0.54 \mathrm{M}$ in the same corresponding solvent) was added. The mixture resulted in the stated catalyst loadings, base and initial aminoborane concentration of $\mathrm{c}_{\text {ini }}=0.27 \mathrm{~mol} / \mathrm{L}$. The reaction was stirred $(800-830 \mathrm{rpm})$ for $16 \mathrm{~h}$ at room temperature in a water bath $\left(23^{\circ} \mathrm{C}\right)$ and was subsequently monitored via ${ }^{11} \mathrm{~B}\left\{{ }^{1} \mathrm{H}\right\}$-NMR spectroscopy. Results of the catalysis were determined by integration of the characteristic ${ }^{11} \mathrm{~B}\left\{{ }^{1} \mathrm{H}\right\}$-NMR-signals. Poisoning experiments were conducted with a $0.54 \mathrm{M}$ stock solution of DBCOT in toluene, a $0.8 \mathrm{M}$ solution of $\mathrm{PMe}_{3}$ in THF or $\mathrm{Hg}$ ( 3 droplets from a syringe with $0.9 \mathrm{~mm}$ cannula) respectively.

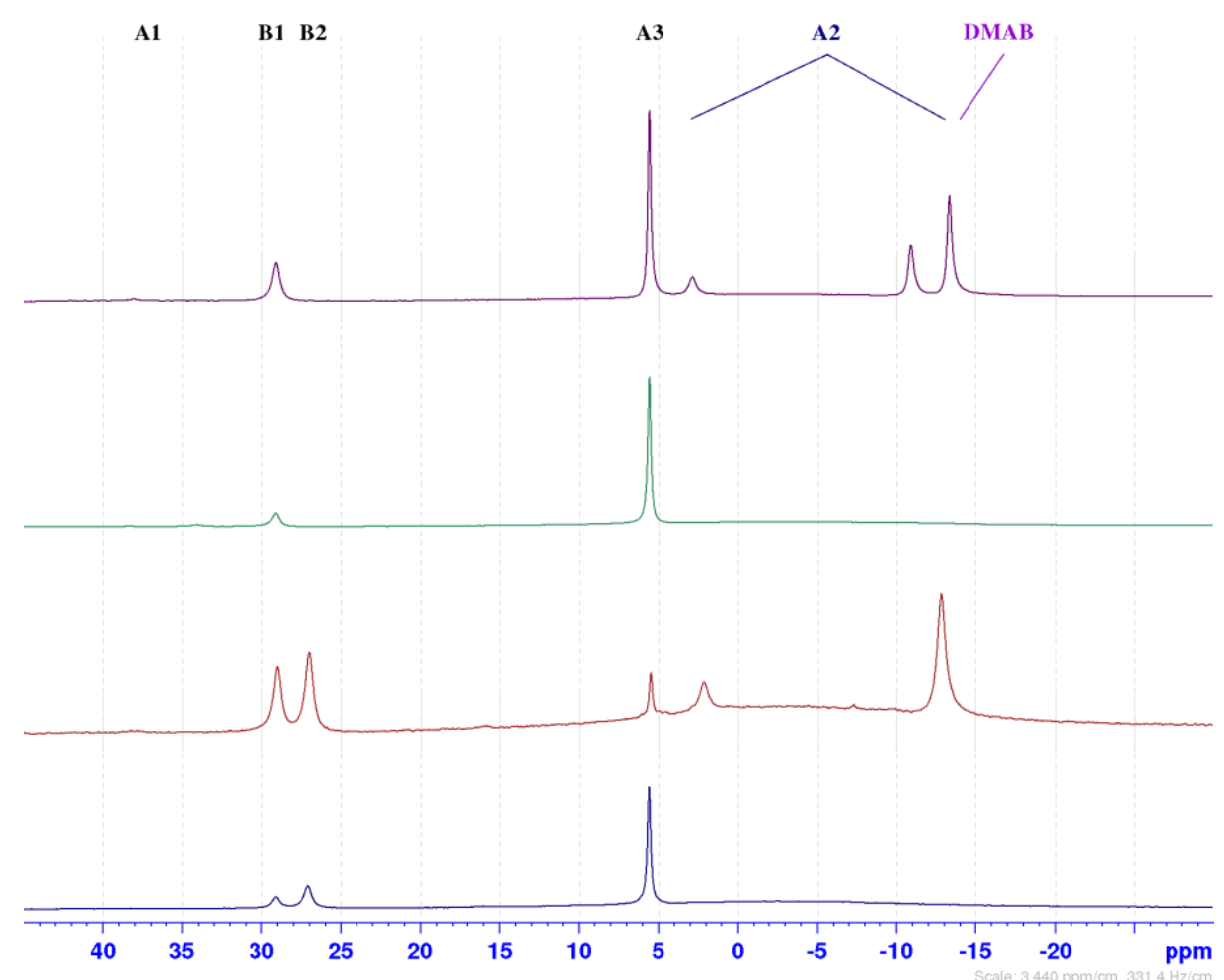

Figure S1 Stacked ${ }^{11} \mathrm{~B}\left\{{ }^{1} \mathrm{H}\right\}$ NMR- spectra from dehydrogenation experiments of DMAB $([\mathrm{Fe}] / \mathrm{B} / \mathrm{S}(/ \mathrm{I})-$ ratios of $0 / 1 / 5$ with LiHMDS at $33^{\circ} \mathrm{C}$ (lilac), $1 / 10 / 50$ with LiHMDS at $33^{\circ} \mathrm{C}$ (green), $1 / 10 / 50 /\left(1 \mathrm{PMe}_{3}\right)$ with $\mathrm{KO}^{t} \mathrm{Bu}$ at $23^{\circ} \mathrm{C}$ (red) and $1 / 10 / 50$ with $\mathrm{KO}^{t} \mathrm{Bu}$ at $23^{\circ} \mathrm{C}$ (blue). 


\section{NMR spectra}

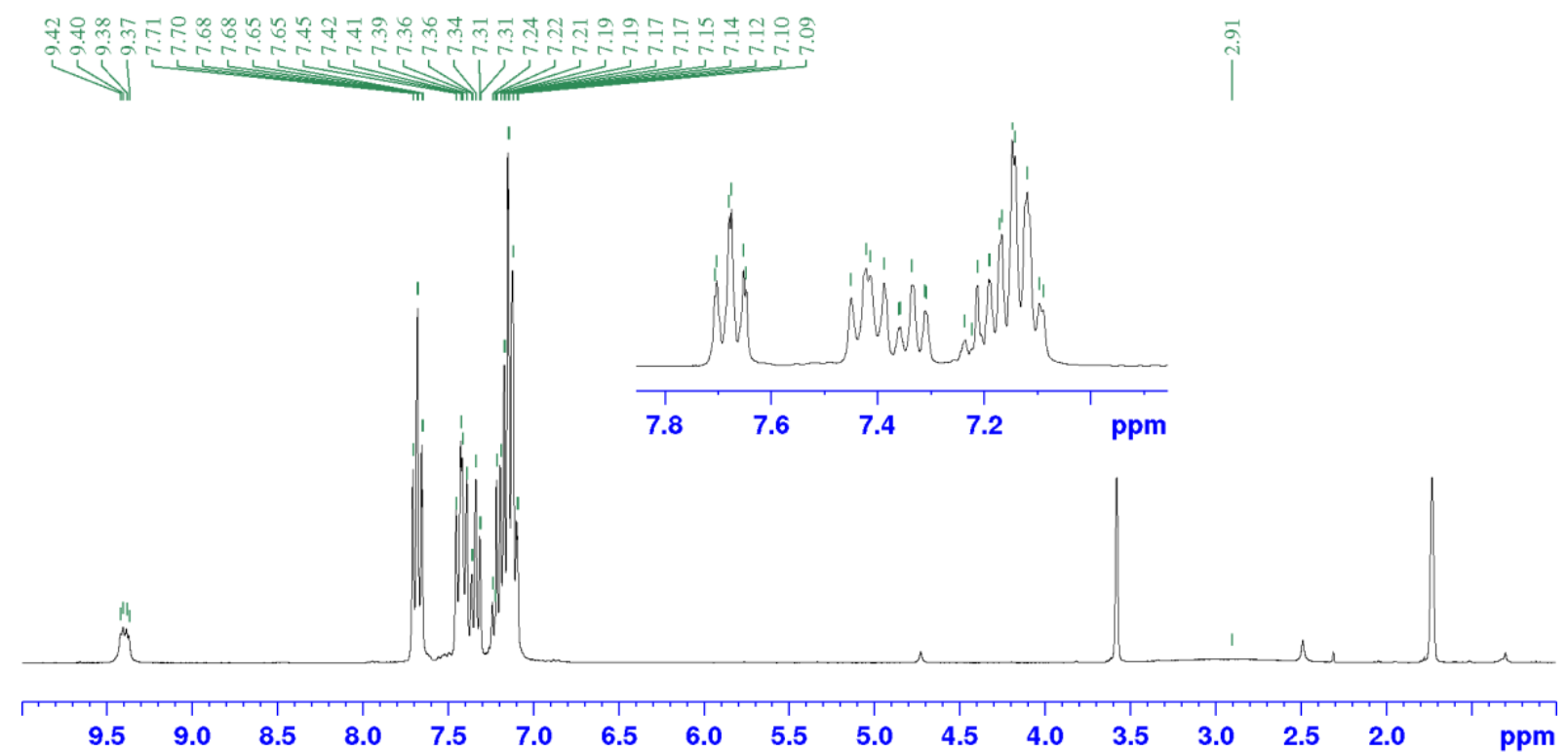

Figure S2 ${ }^{1} \mathrm{H}-\mathrm{NMR}$ spectrum (300 MHz, d8-THF) of $\mathbf{1 a}$.

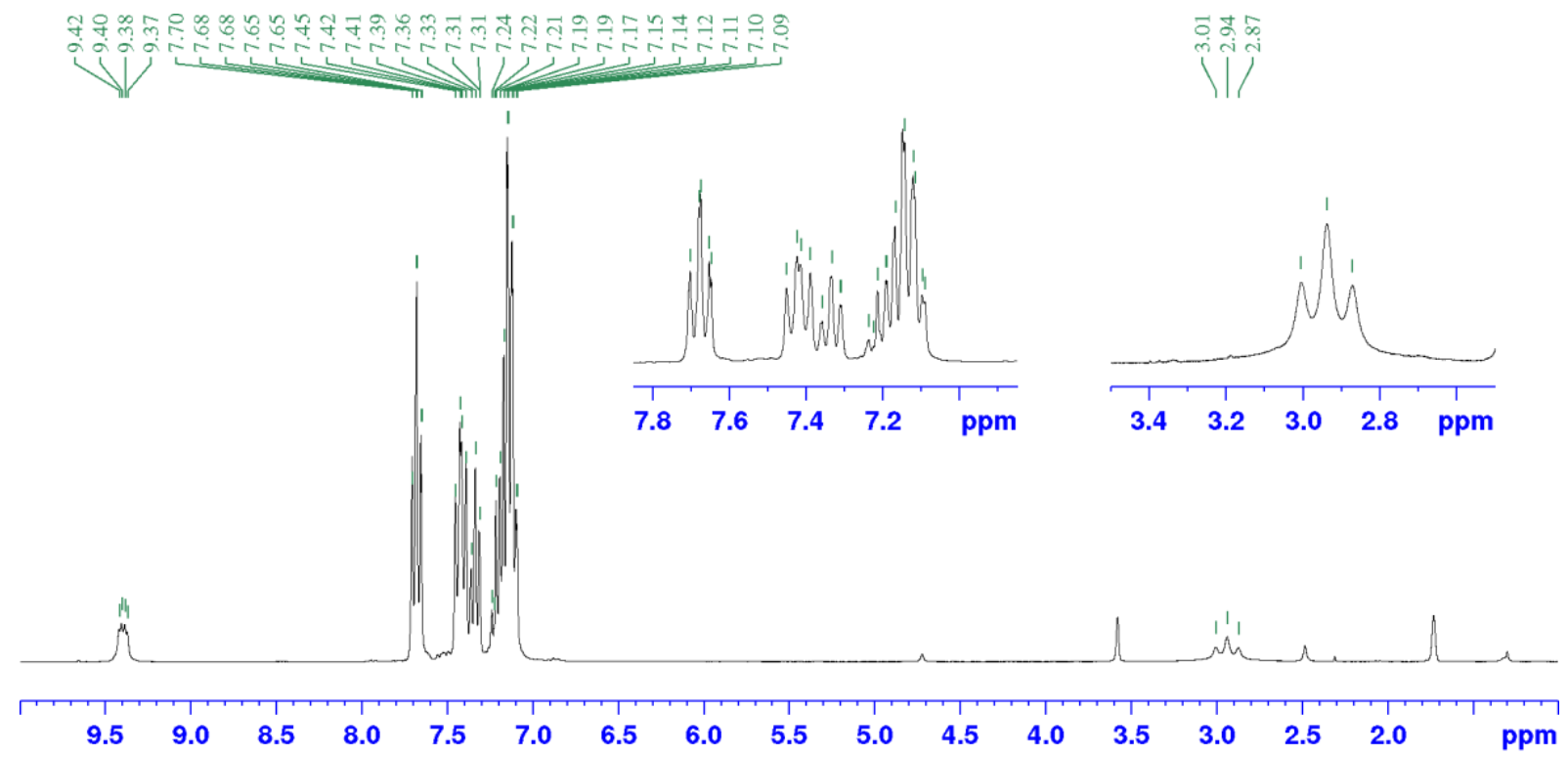

Figure $\mathbf{S 3}{ }^{1} \mathrm{H}\left\{{ }^{11} \mathrm{~B}\right\}-\mathrm{NMR}$ spectrum (300 MHz, d8-THF) of 1a. 


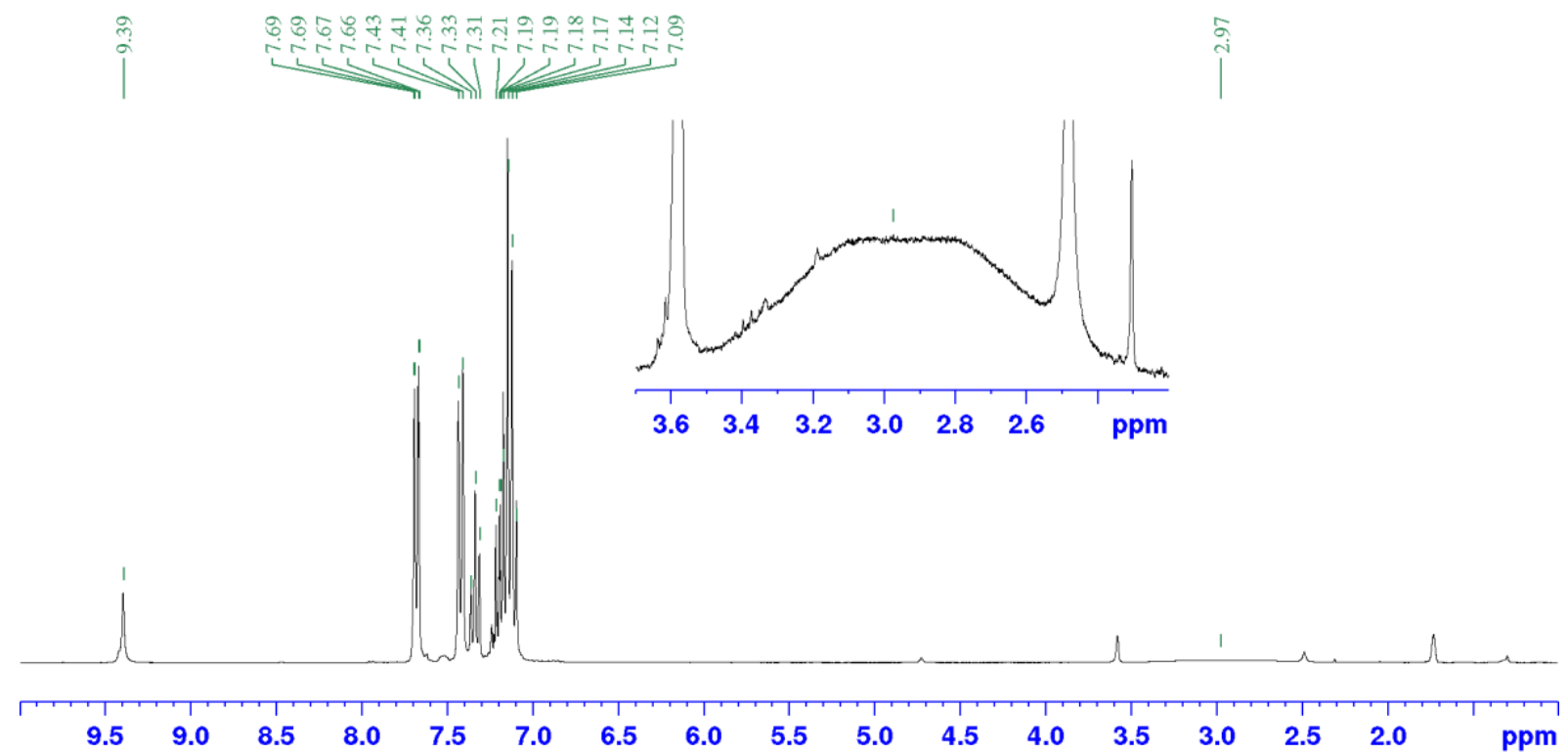

Figure $\mathbf{S 4}{ }^{1} \mathrm{H}\left\{{ }^{31} \mathrm{P}\right\}$-NMR spectrum (300 MHz, $\mathrm{d}_{8}$-THF) of $\mathbf{1 a}$.

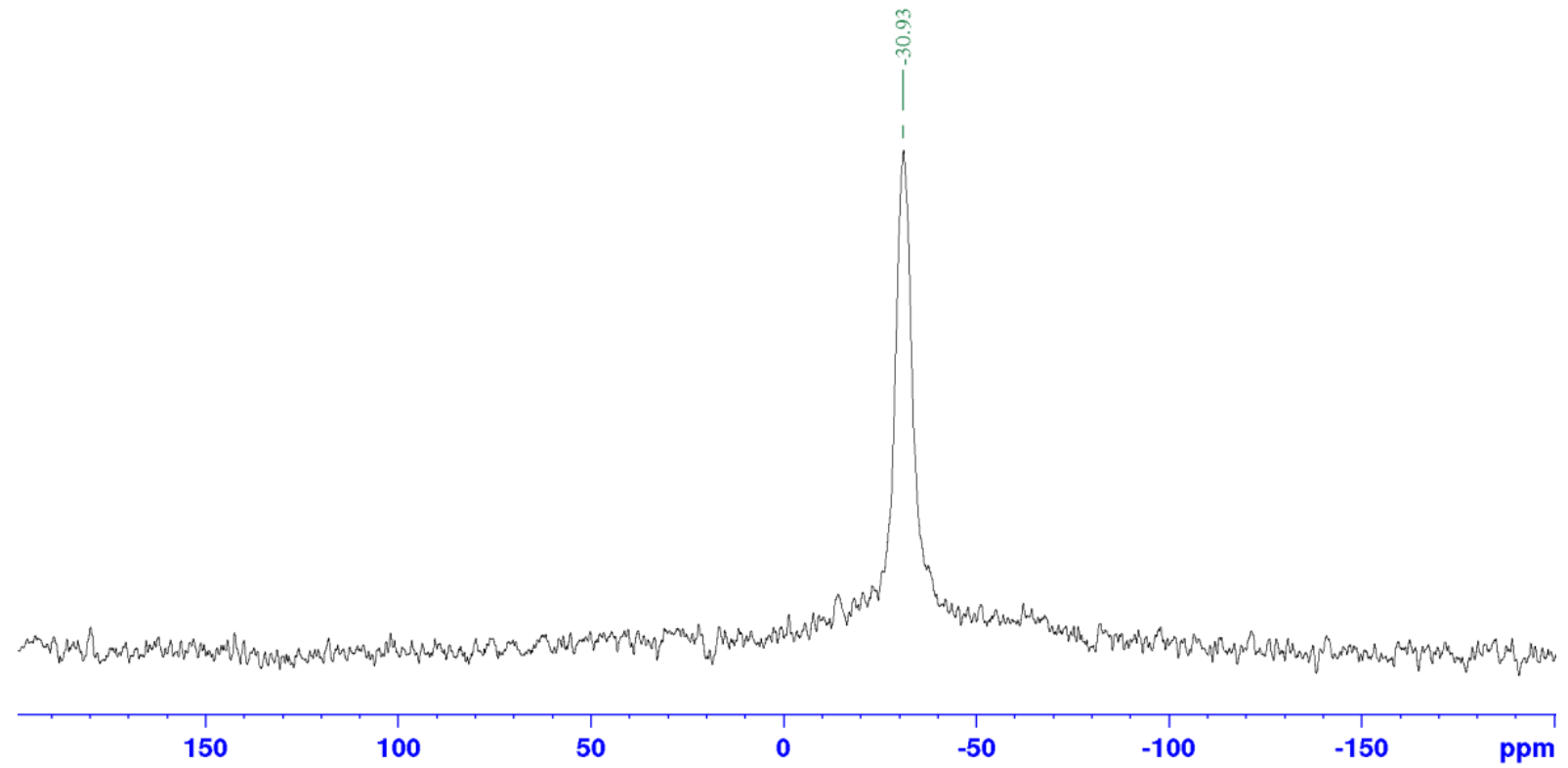

Figure $\mathbf{S 5}{ }^{11} \mathrm{~B}\left\{{ }^{1} \mathrm{H}\right\}$-NMR spectrum $\left(96 \mathrm{MHz}, \mathrm{d}_{8}\right.$-THF) of $\mathbf{1 a}$. 


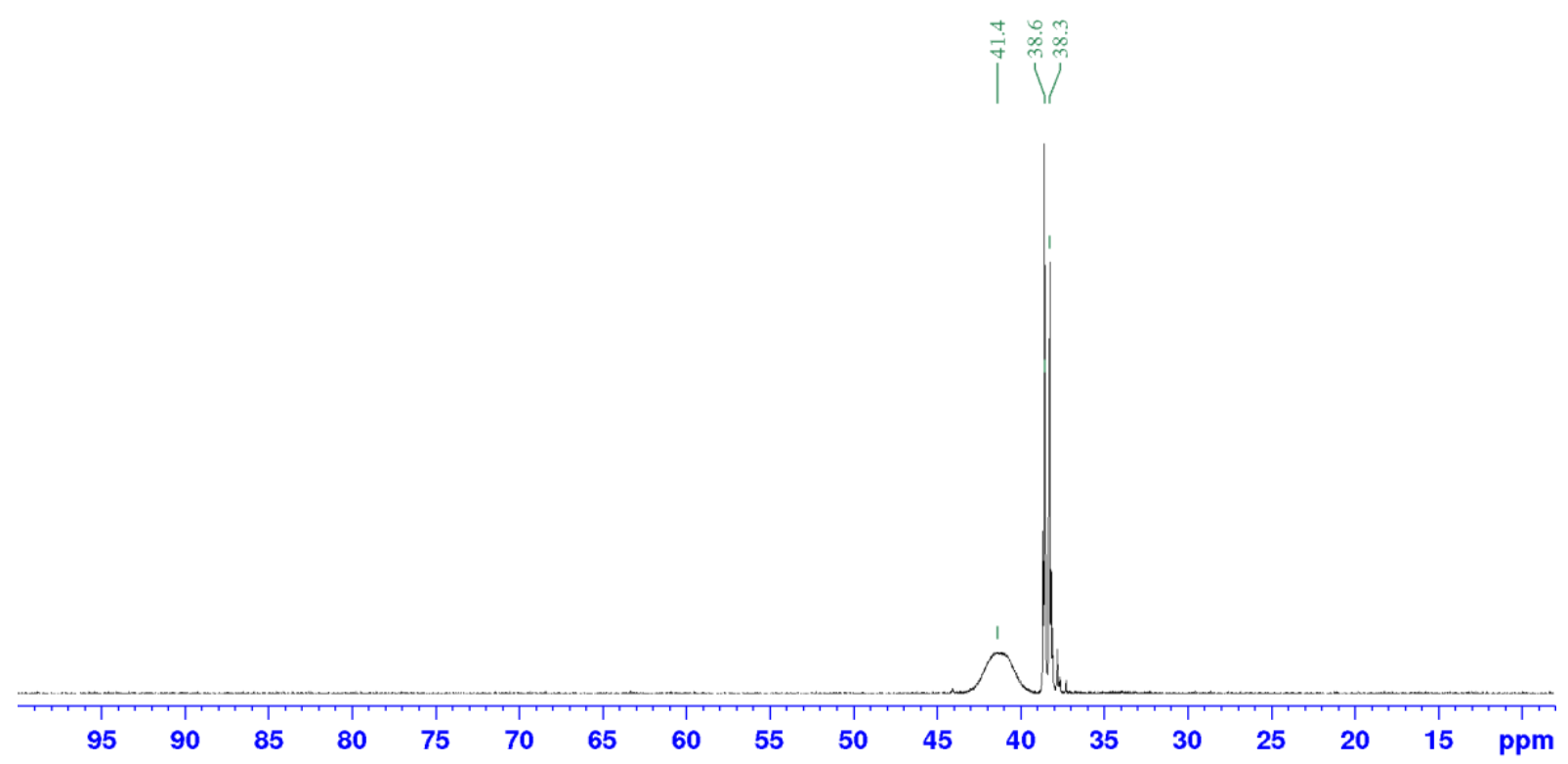

Figure S6 ${ }^{31} \mathrm{P}\left\{{ }^{1} \mathrm{H}\right\}$-NMR spectrum (122 MHz, $\mathrm{d}_{8}$-THF) of $\mathbf{1 a}$.

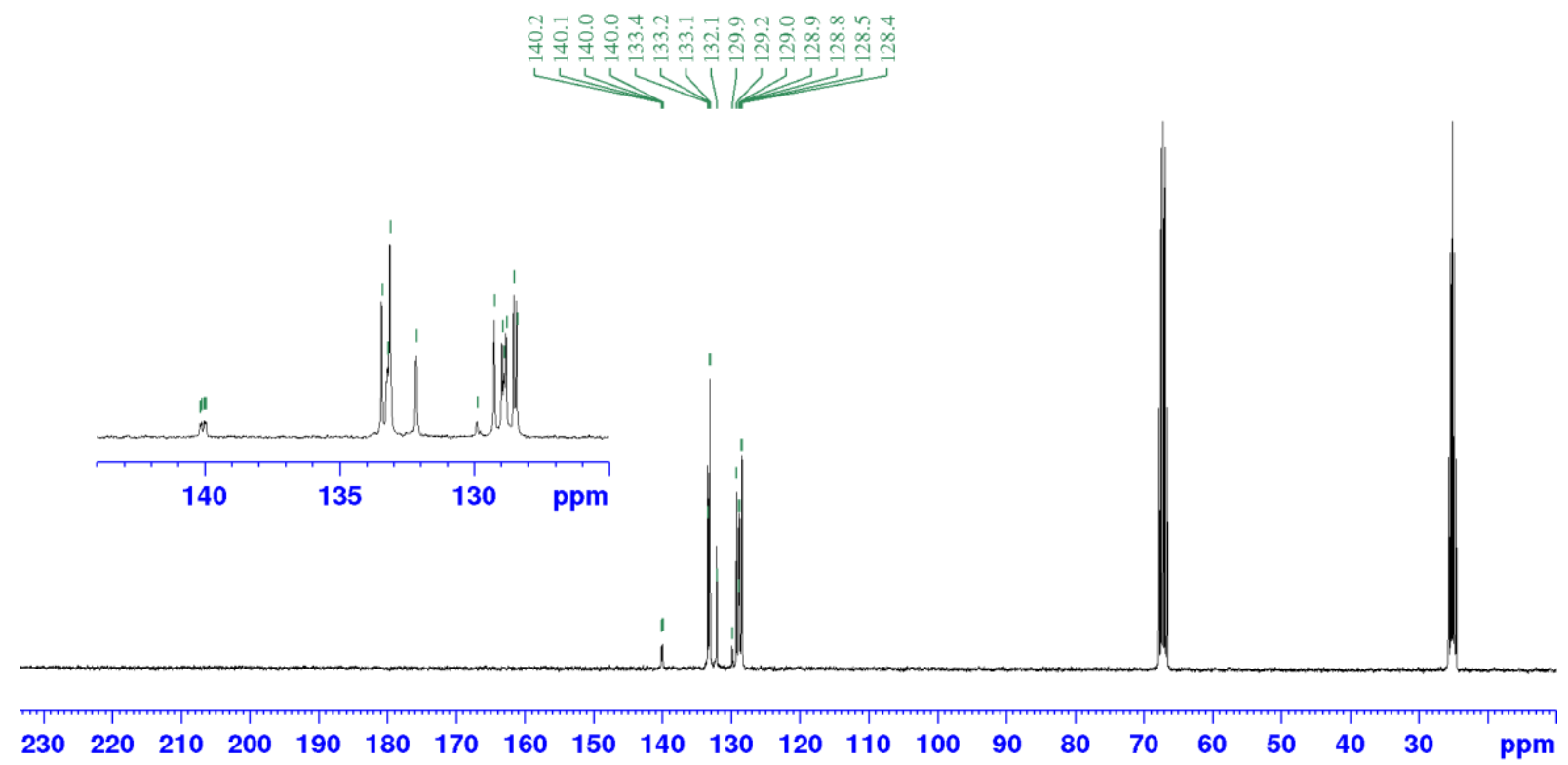

Figure $\mathbf{S 7}{ }^{13} \mathrm{C}-\mathrm{NMR}$ spectrum $\left(76 \mathrm{MHz}, \mathrm{d}_{8}-\mathrm{THF}\right)$ of $\mathbf{1 a}$. 


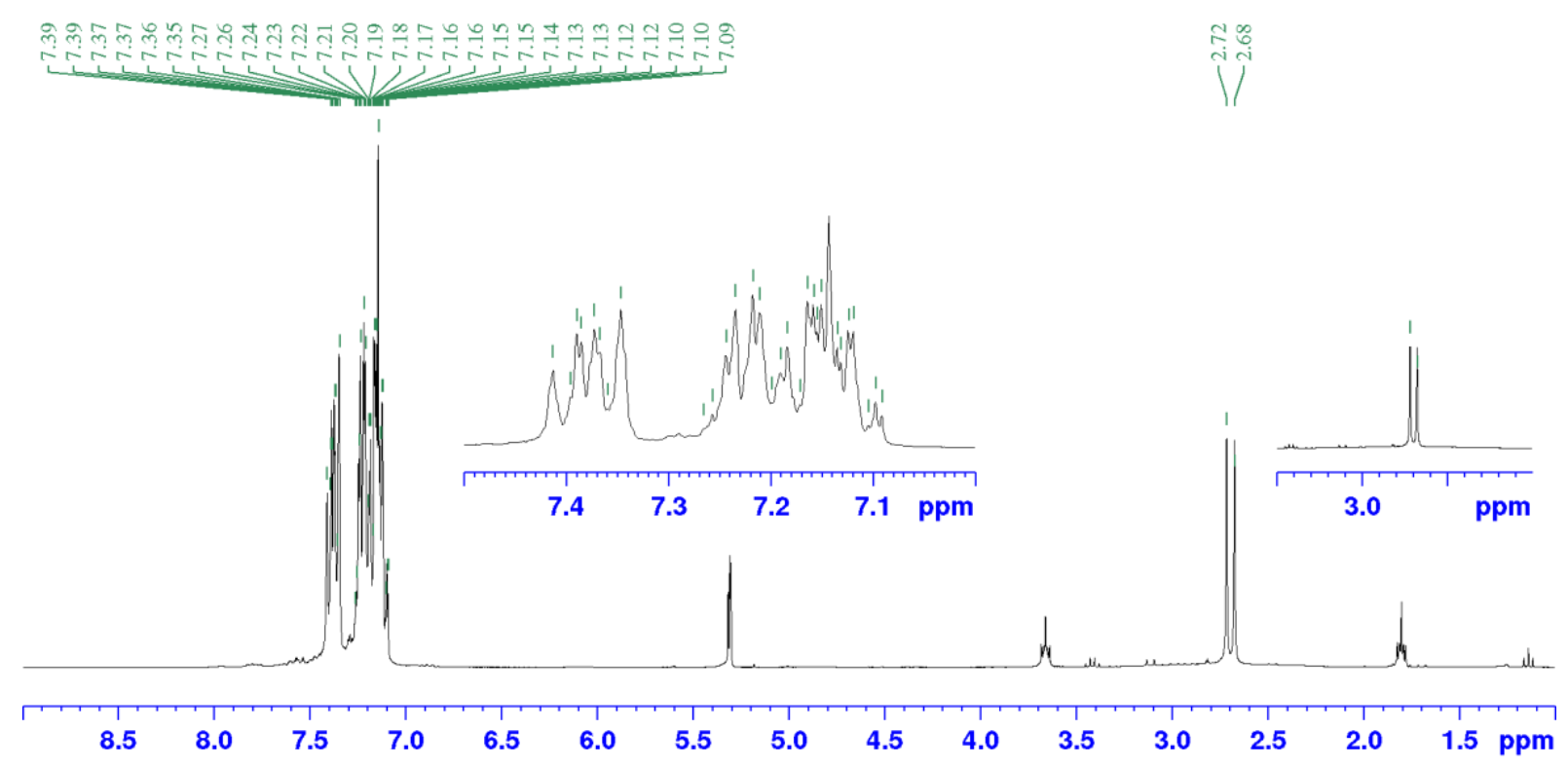

Figure S8 ${ }^{1} \mathrm{H}-\mathrm{NMR}$ spectrum $\left(300 \mathrm{MHz}, \mathrm{CD}_{2} \mathrm{Cl}_{2}\right)$ of $\mathbf{1 b}$.

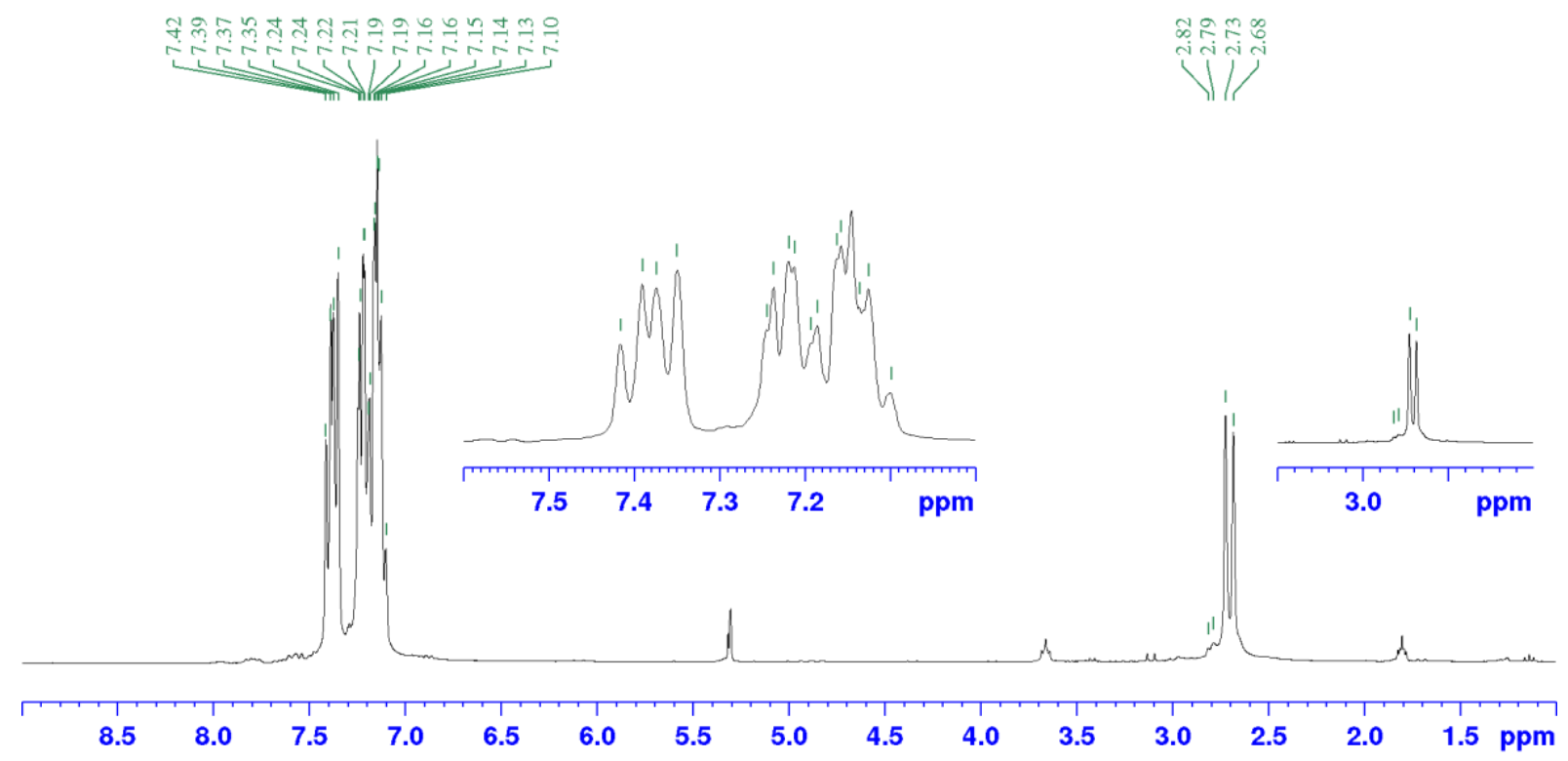

Figure $\mathbf{S 9}{ }^{1} \mathrm{H}\left\{{ }^{11} \mathrm{~B}\right\}$-NMR spectrum $\left(300 \mathrm{MHz}, \mathrm{CD}_{2} \mathrm{Cl}_{2}\right)$ of $\mathbf{1 b}$. 


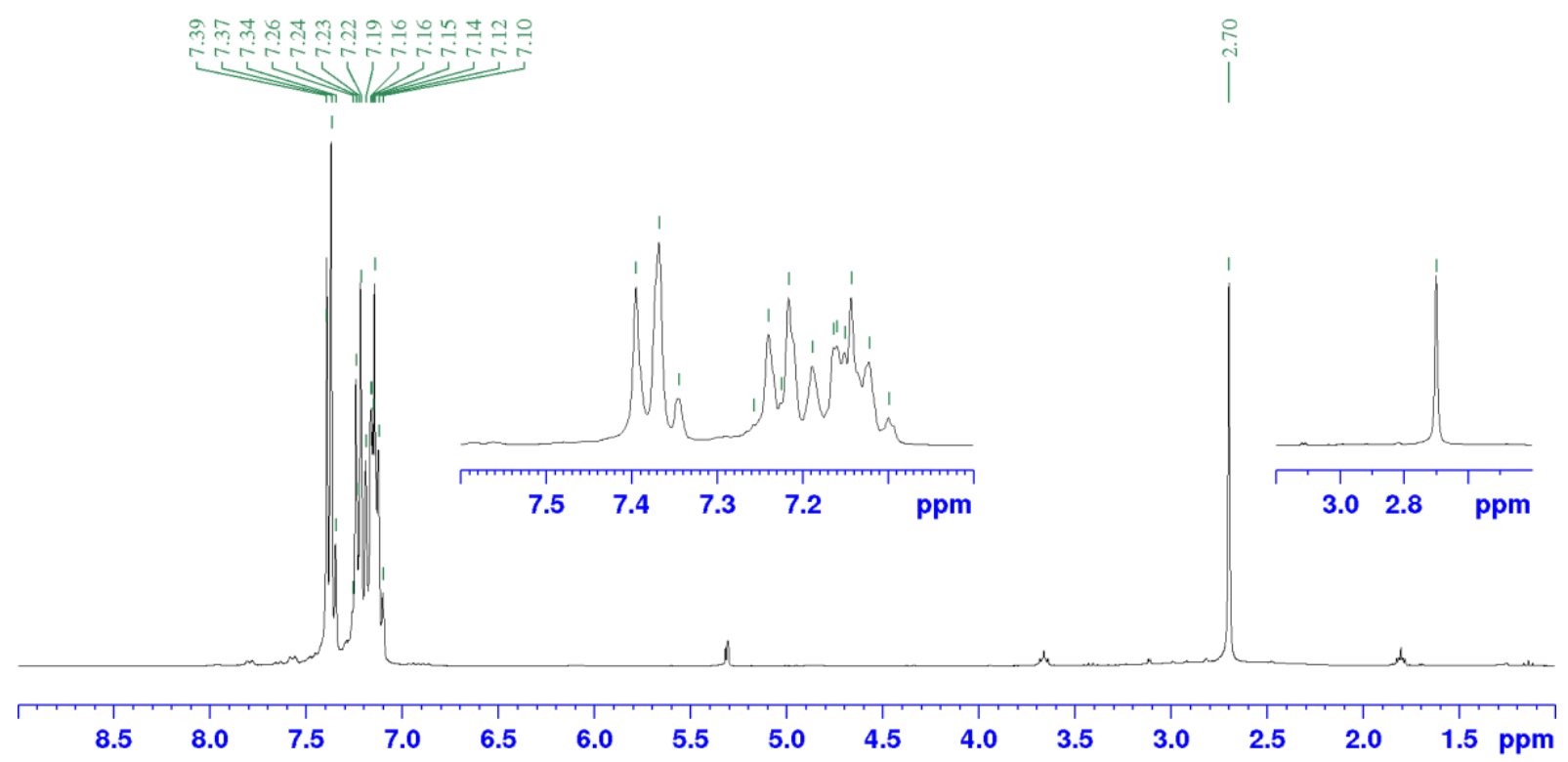

Figure $\mathbf{S 1 0}{ }^{1} \mathrm{H}\left\{{ }^{31} \mathrm{P}\right\}$-NMR spectrum $\left(300 \mathrm{MHz}, \mathrm{CD}_{2} \mathrm{Cl}_{2}\right)$ of $\mathbf{1 b}$.

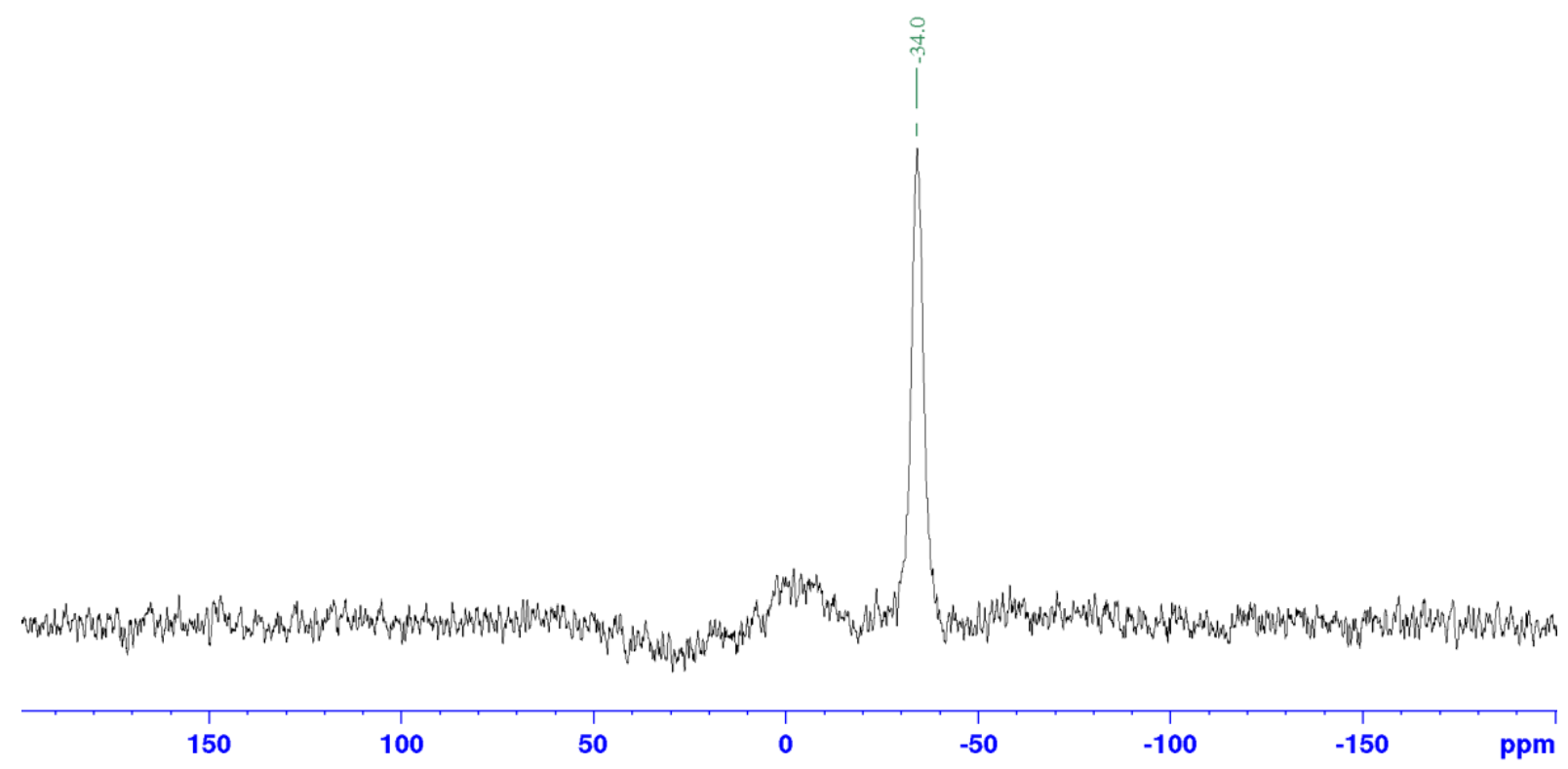

Figure $\mathbf{S 1 1}{ }^{11} \mathrm{~B}\left\{{ }^{1} \mathrm{H}\right\}$-NMR spectrum $\left(96 \mathrm{MHz}, \mathrm{CD}_{2} \mathrm{Cl}_{2}\right)$ of $\mathbf{1 b}$. 


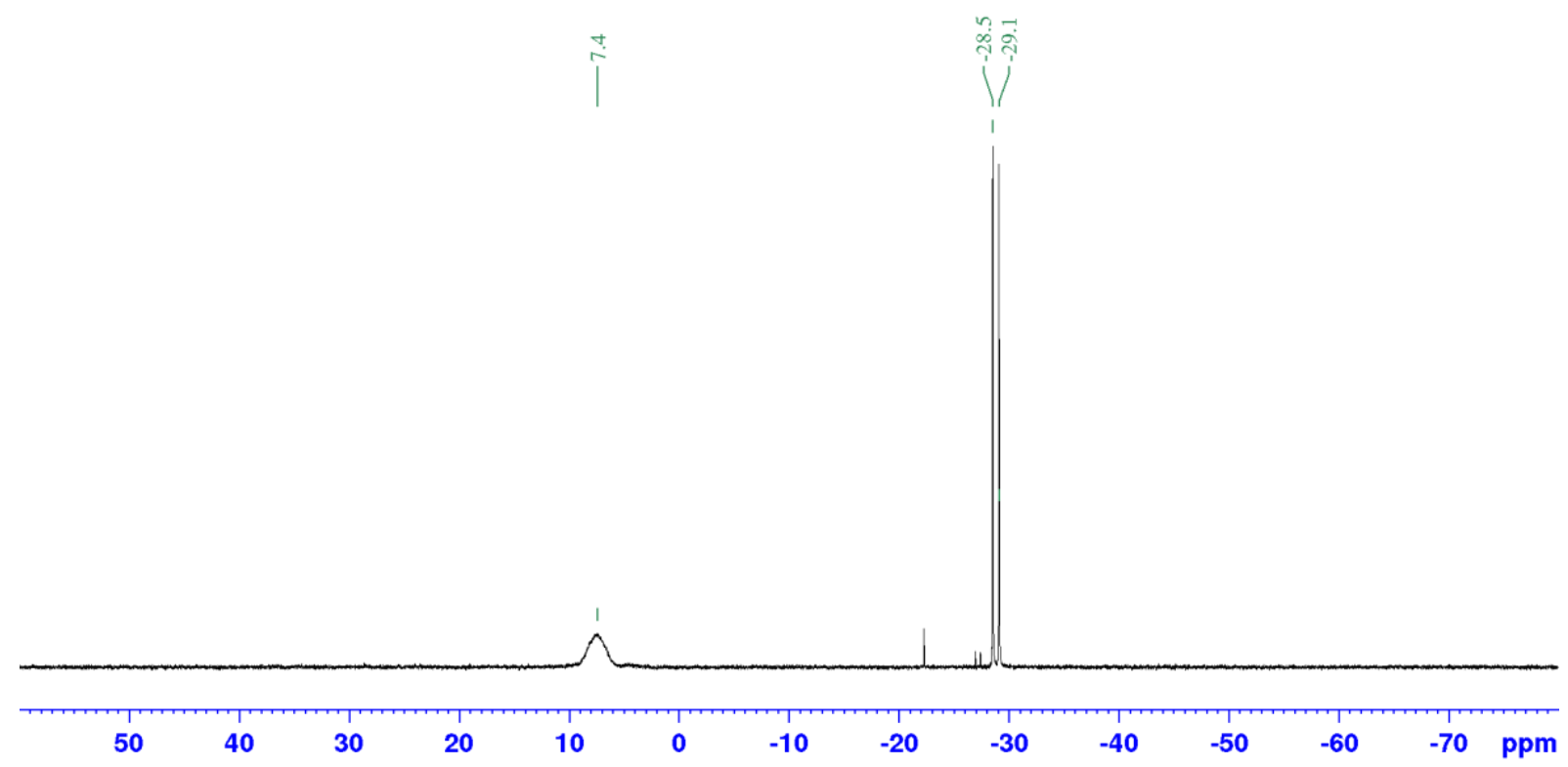

Figure $\mathbf{S 1 2}{ }^{31} \mathrm{P}\left\{{ }^{1} \mathrm{H}\right\}$-NMR spectrum (122 MHz, $\left.\mathrm{CD}_{2} \mathrm{Cl}_{2}\right)$ of $\mathbf{1 b}$.

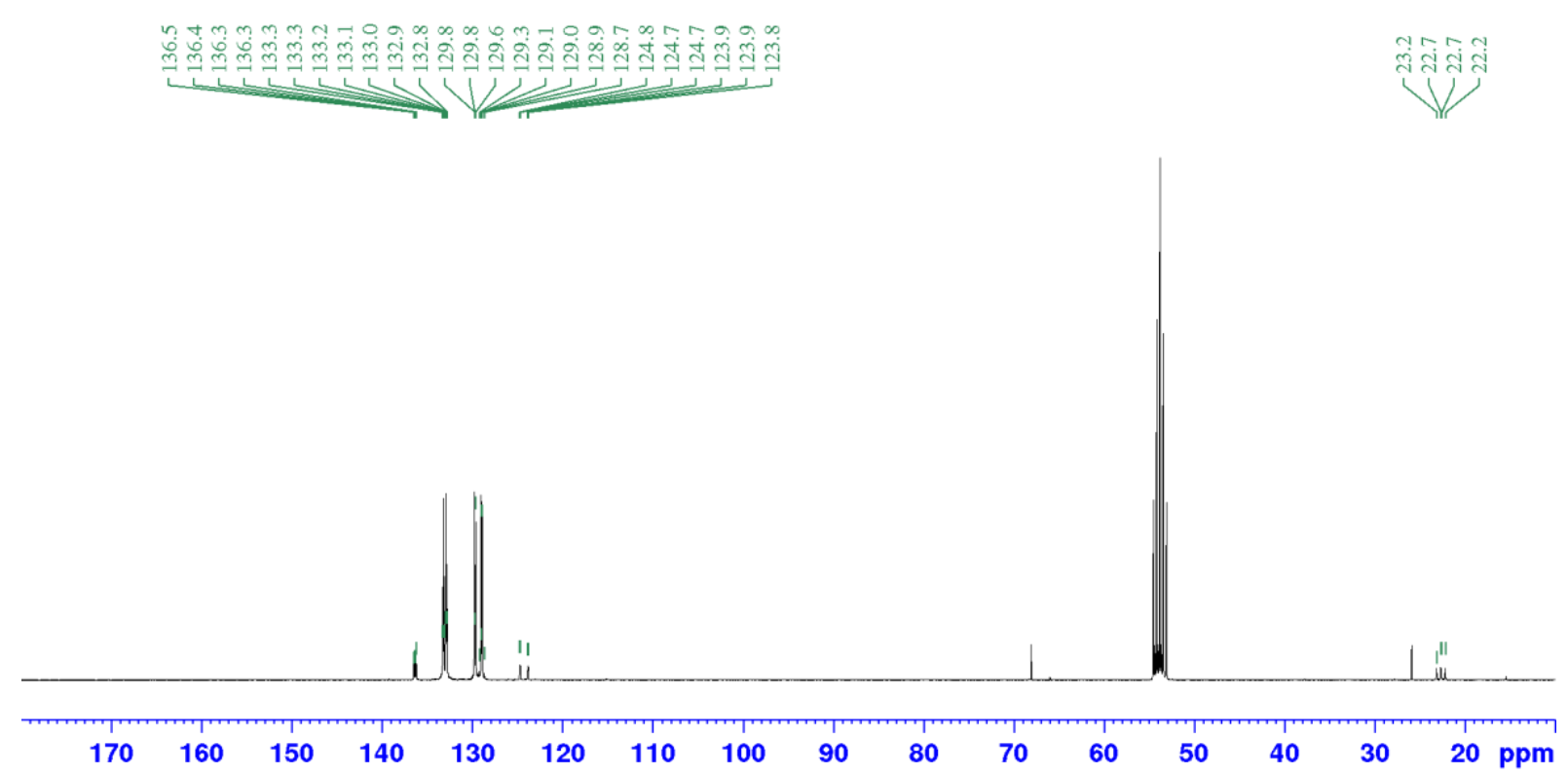

Figure $\mathbf{S 1 3}{ }^{13} \mathrm{C}-\mathrm{NMR}$ spectrum $\left(75 \mathrm{MHz}, \mathrm{CD}_{2} \mathrm{Cl}_{2}\right)$ of $\mathbf{1 b}$. 

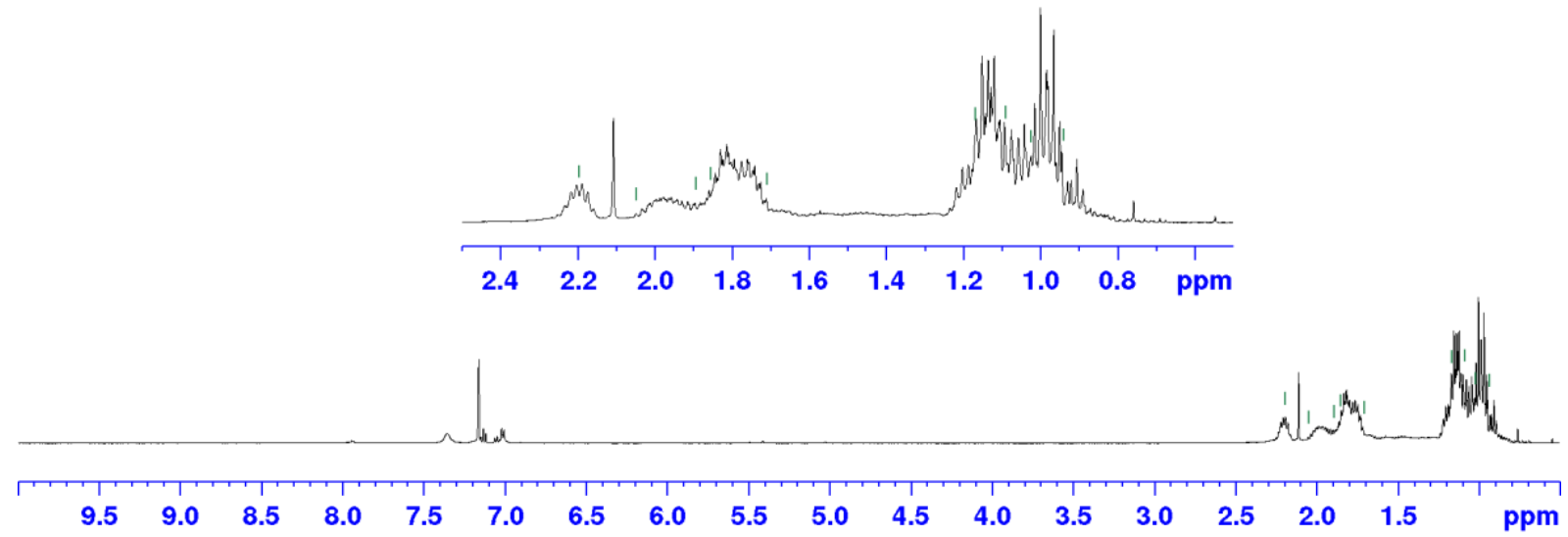

Figure S14 ${ }^{1} \mathrm{H}-\mathrm{NMR}$ spectrum $\left(500 \mathrm{MHz}, \mathrm{C}_{6} \mathrm{D}_{6}\right)$ of $\mathbf{1 c}$.

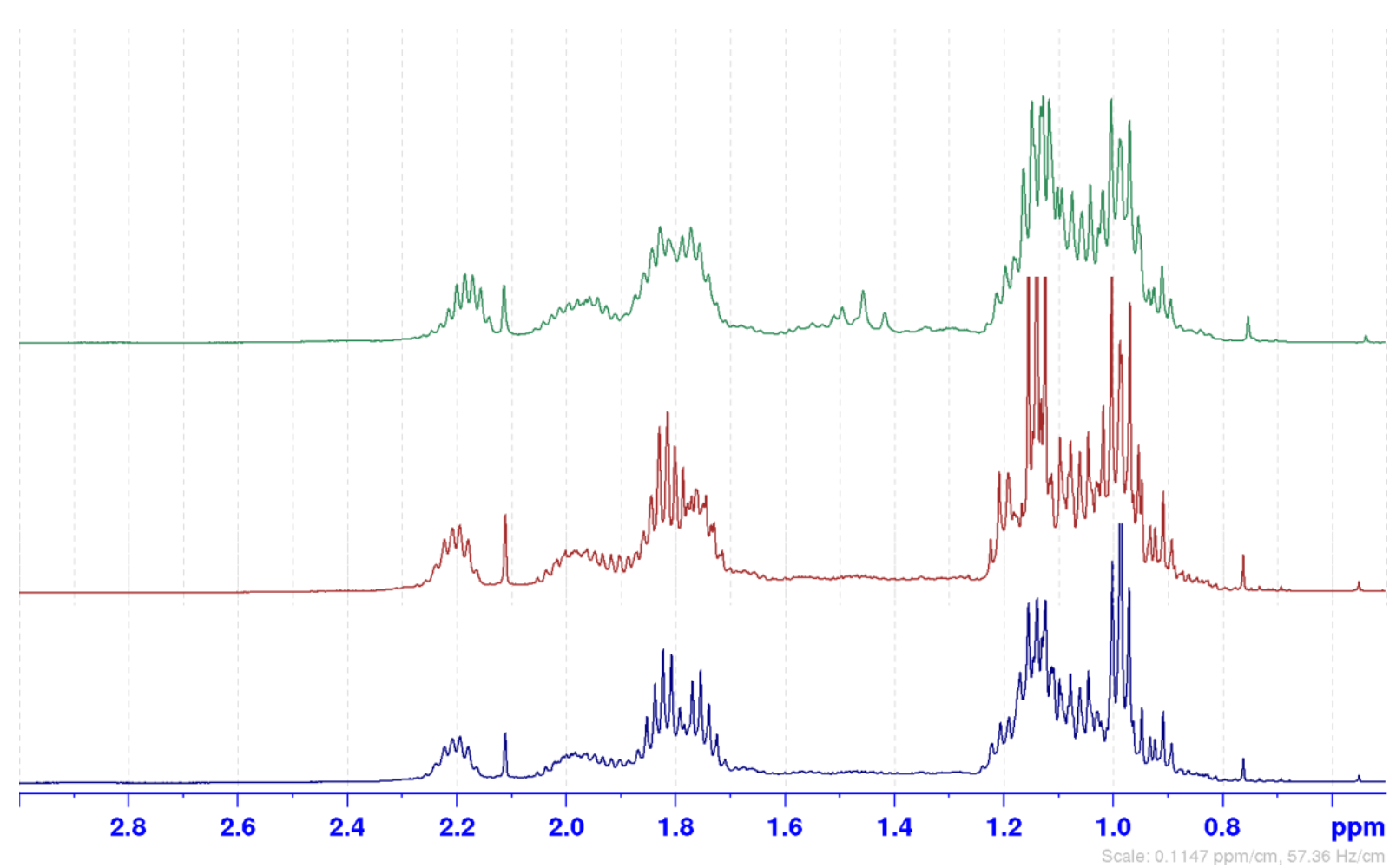

Figure $\mathbf{S 1 5}{ }^{1} \mathrm{H}\left\{{ }^{11} \mathrm{~B}\right\}$-NMR (green) and ${ }^{1} \mathrm{H}\left\{{ }^{31} \mathrm{P}\right\}$-NMR spectra (red and blue) (500 MHz, $\mathrm{C}_{6} \mathrm{D}_{6}$ ) of $\mathbf{1 c}$. 


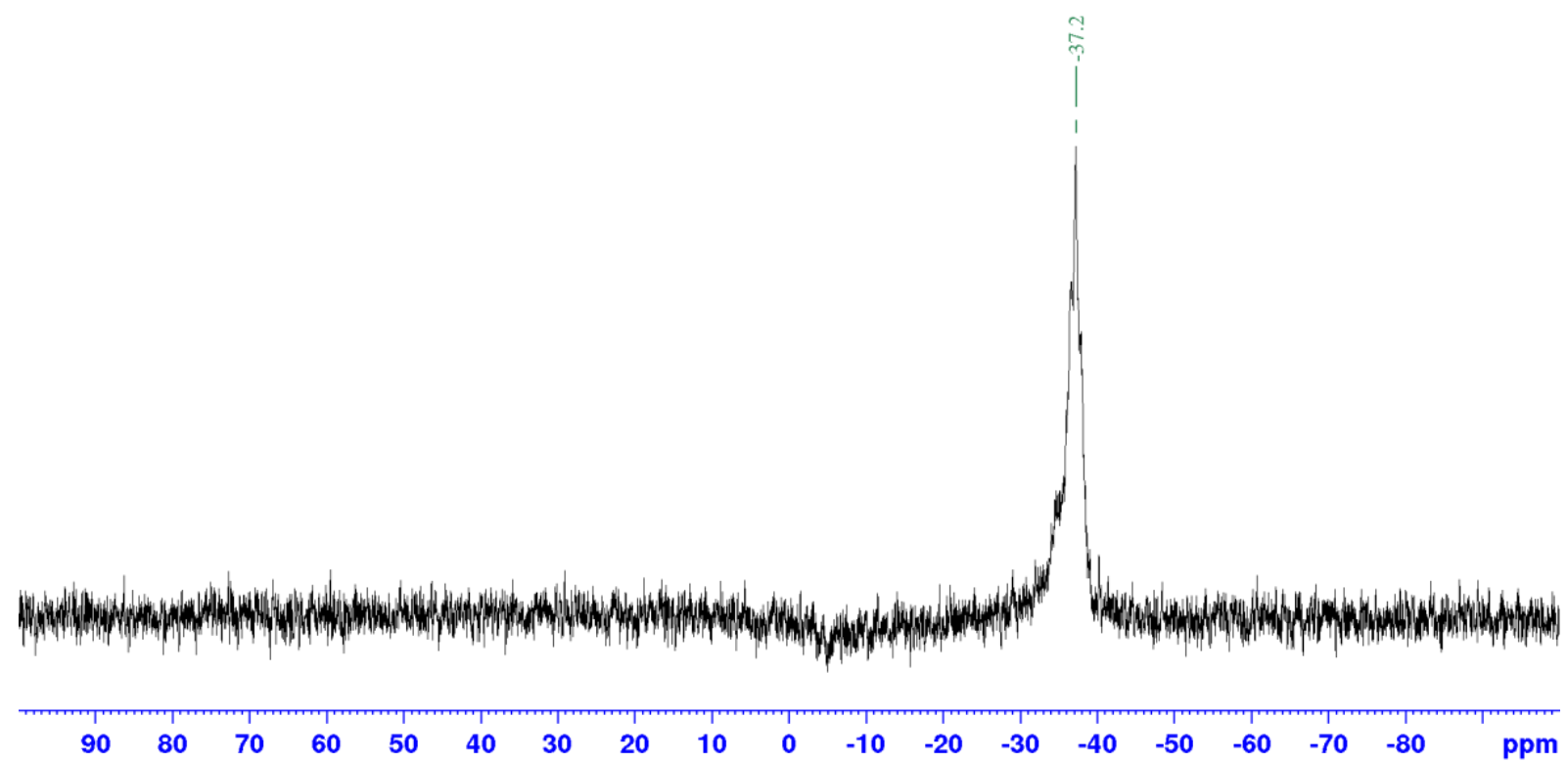

Figure $\mathbf{S 1 6}{ }^{11} \mathrm{~B}\left\{{ }^{1} \mathrm{H}\right\}$-NMR spectrum $\left(161 \mathrm{MHz}, \mathrm{C}_{6} \mathrm{D}_{6}\right)$ of $\mathbf{1 c}$.

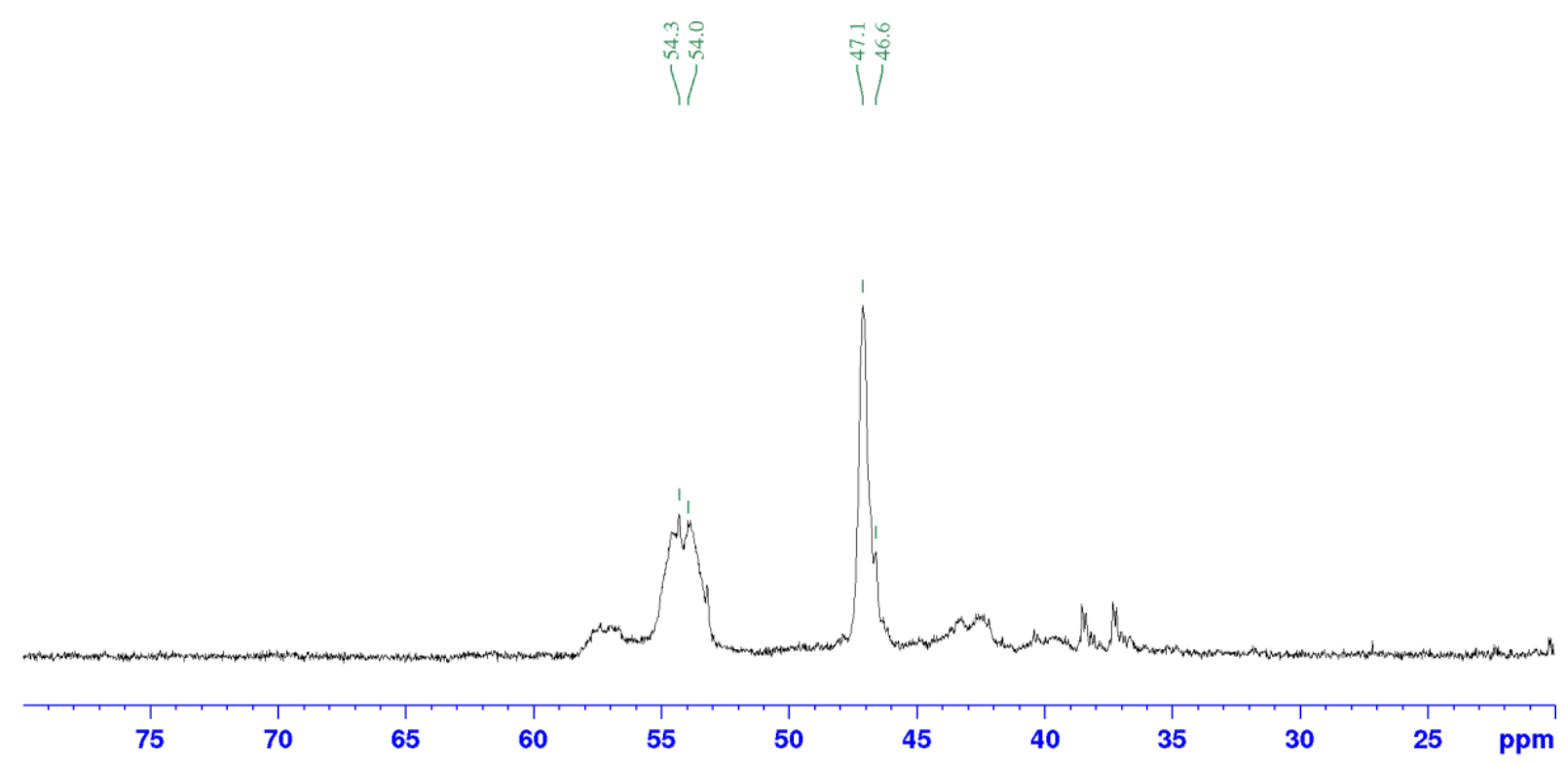

Figure $\mathbf{S 1 7}{ }^{31} \mathrm{P}\left\{{ }^{1} \mathrm{H}\right\}$-NMR spectrum $\left(500 \mathrm{MHz}, \mathrm{C}_{6} \mathrm{D}_{6}\right)$ of $\mathbf{1 c}$. 


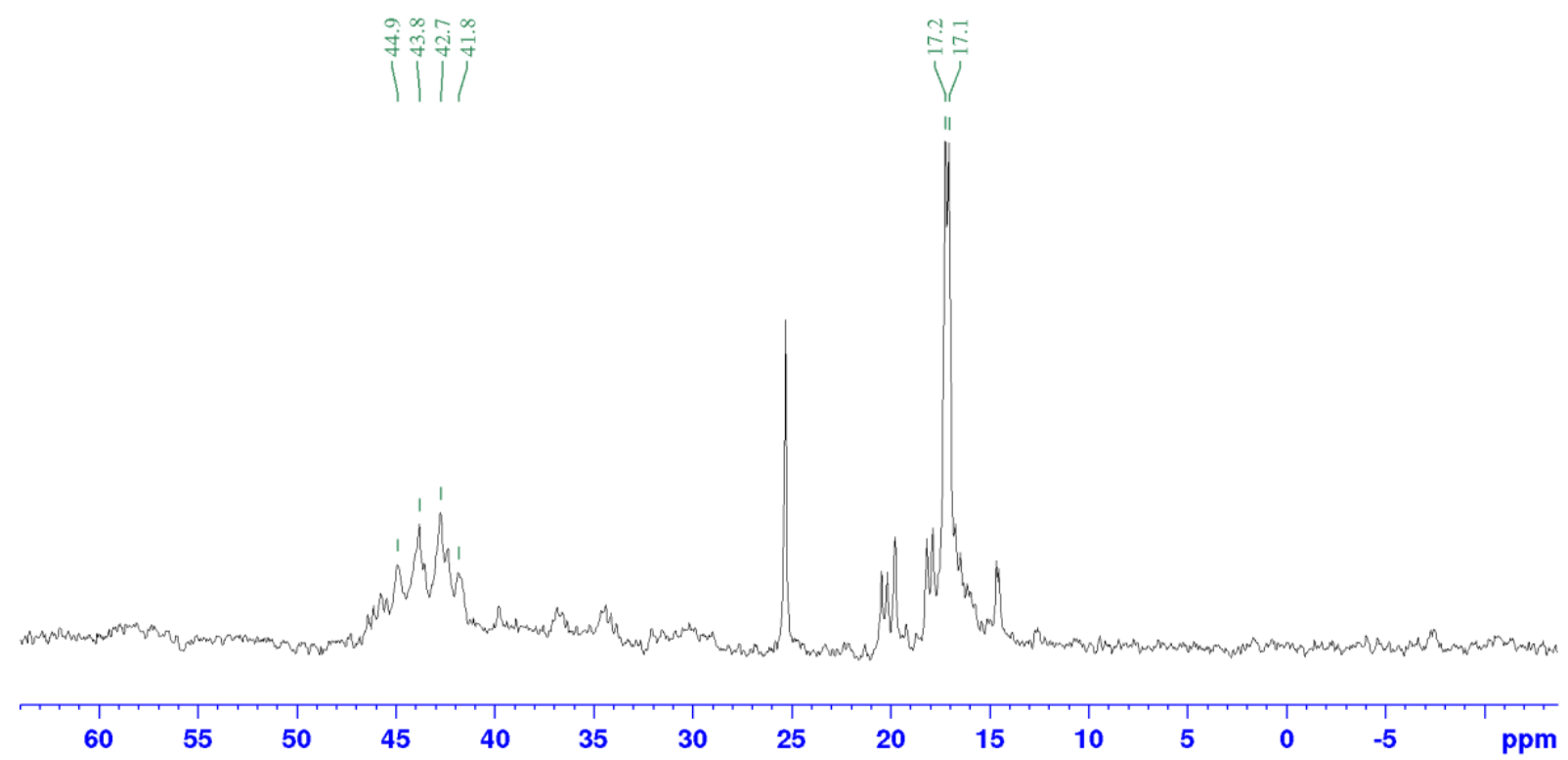

Figure $\mathbf{S 1 8}{ }^{31} \mathrm{P}\left\{{ }^{1} \mathrm{H}\right\}$-NMR spectrum $\left(300 \mathrm{MHz}, \mathrm{CH}_{2} \mathrm{Cl}_{2}\right)$ of $\mathbf{1 c}$.

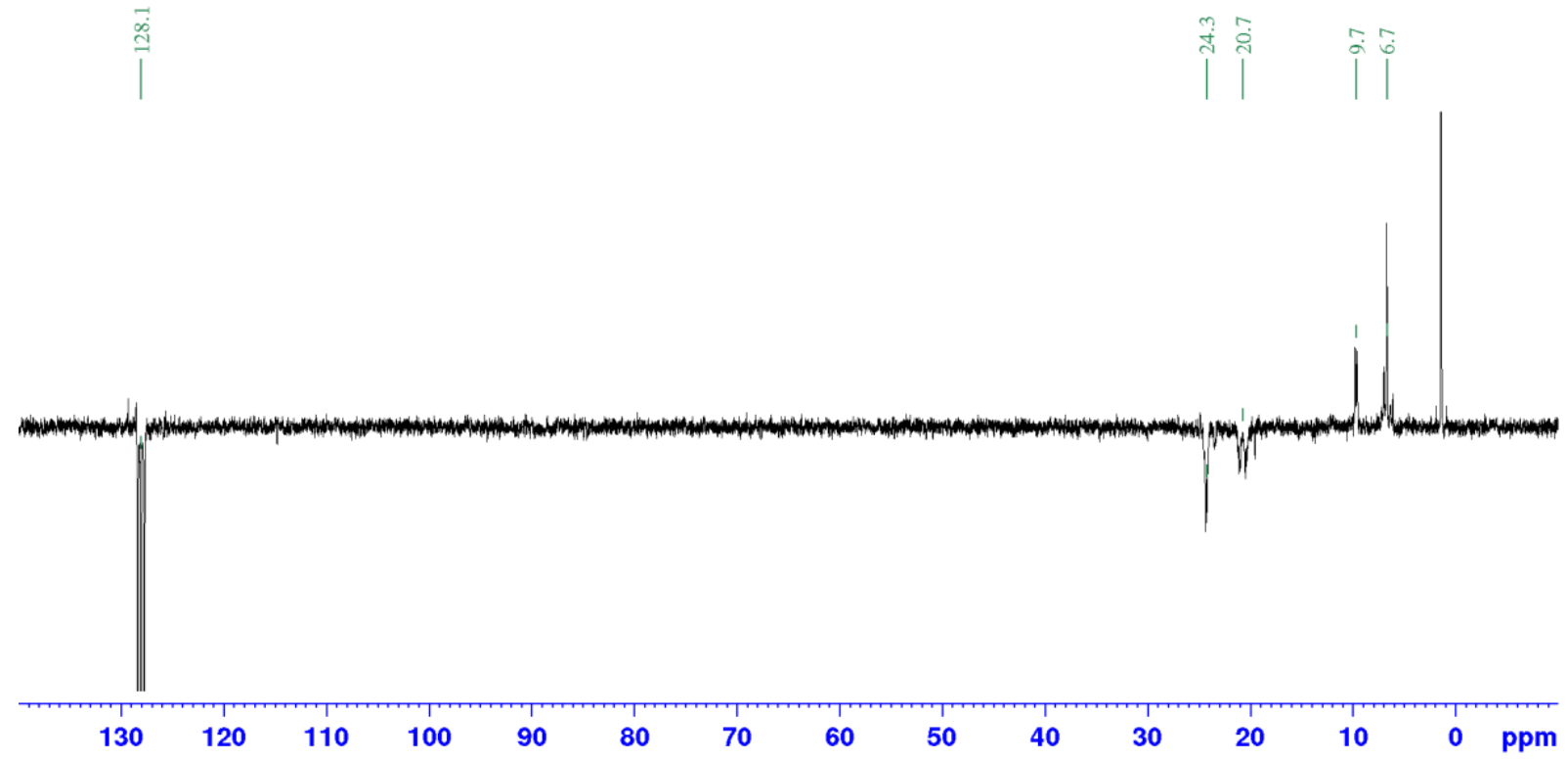

Figure S19 ${ }^{13} \mathrm{C}$-APT-NMR spectrum $\left(76 \mathrm{MHz}, \mathrm{C}_{6} \mathrm{D}_{6}\right)$ of $\mathbf{1 c}$. 


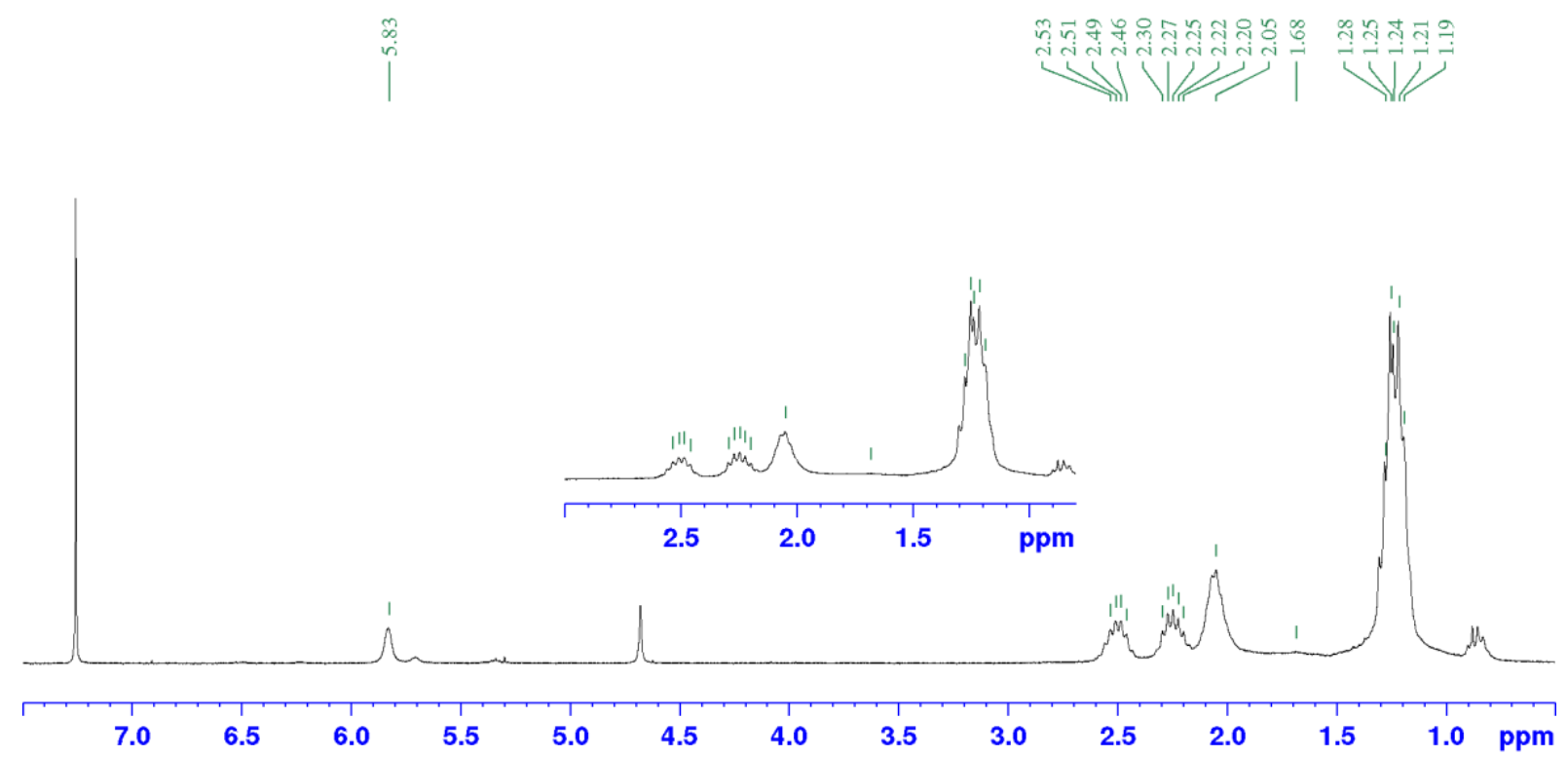

Figure $\mathbf{S 2 0}{ }^{1} \mathrm{H}-\mathrm{NMR}$ spectrum $\left(300 \mathrm{MHz}, \mathrm{CDCl}_{3}\right)$ of $\mathbf{2 c}$.

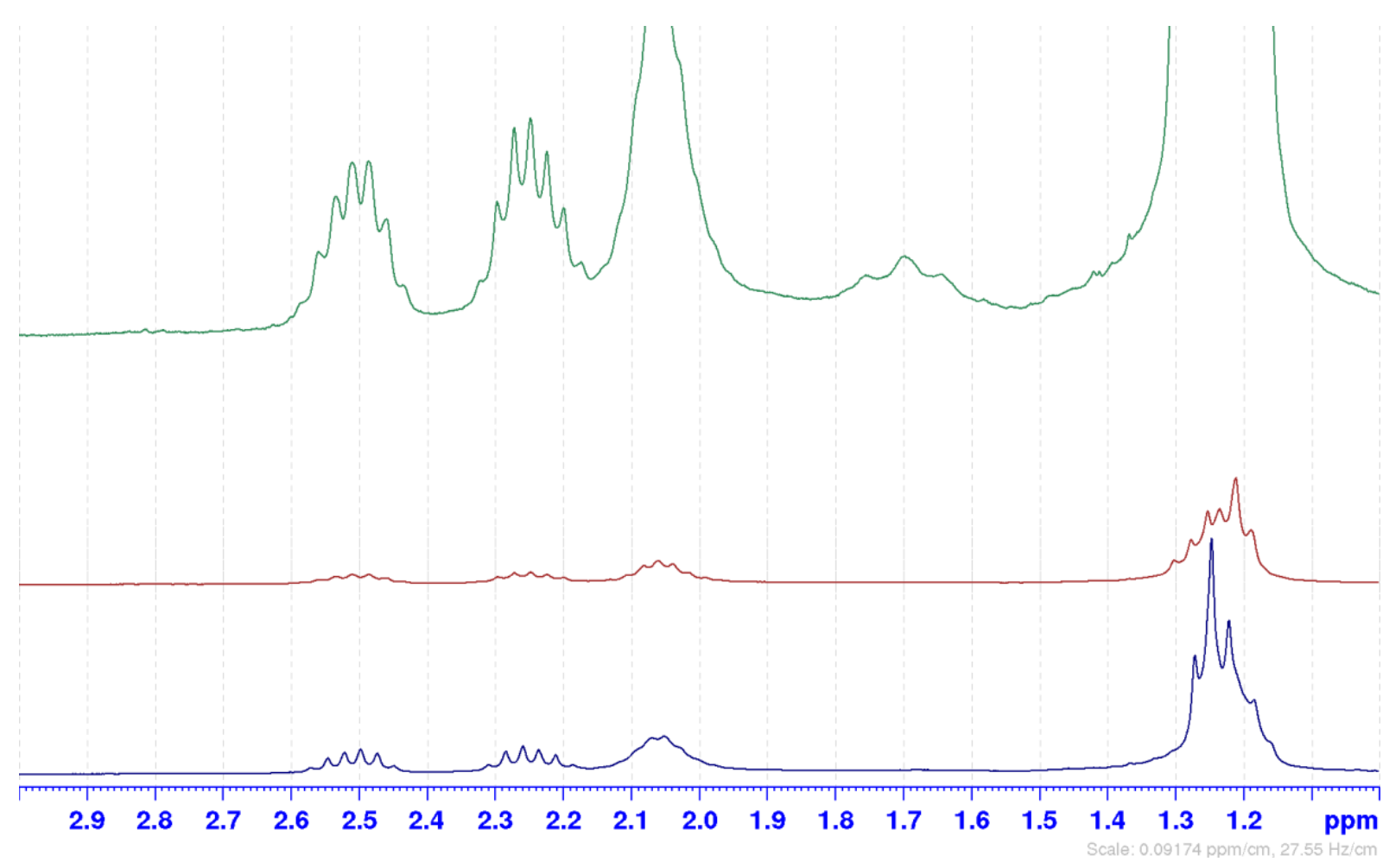

Figure S21 ${ }^{1} \mathrm{H}\left\{{ }^{11} \mathrm{~B}\right\}$-NMR spectrum (green) and ${ }^{1} \mathrm{H}\left\{{ }^{31} \mathrm{P}\right\}$-NMR spectra (blue and red) of $\mathbf{2 c}$. 


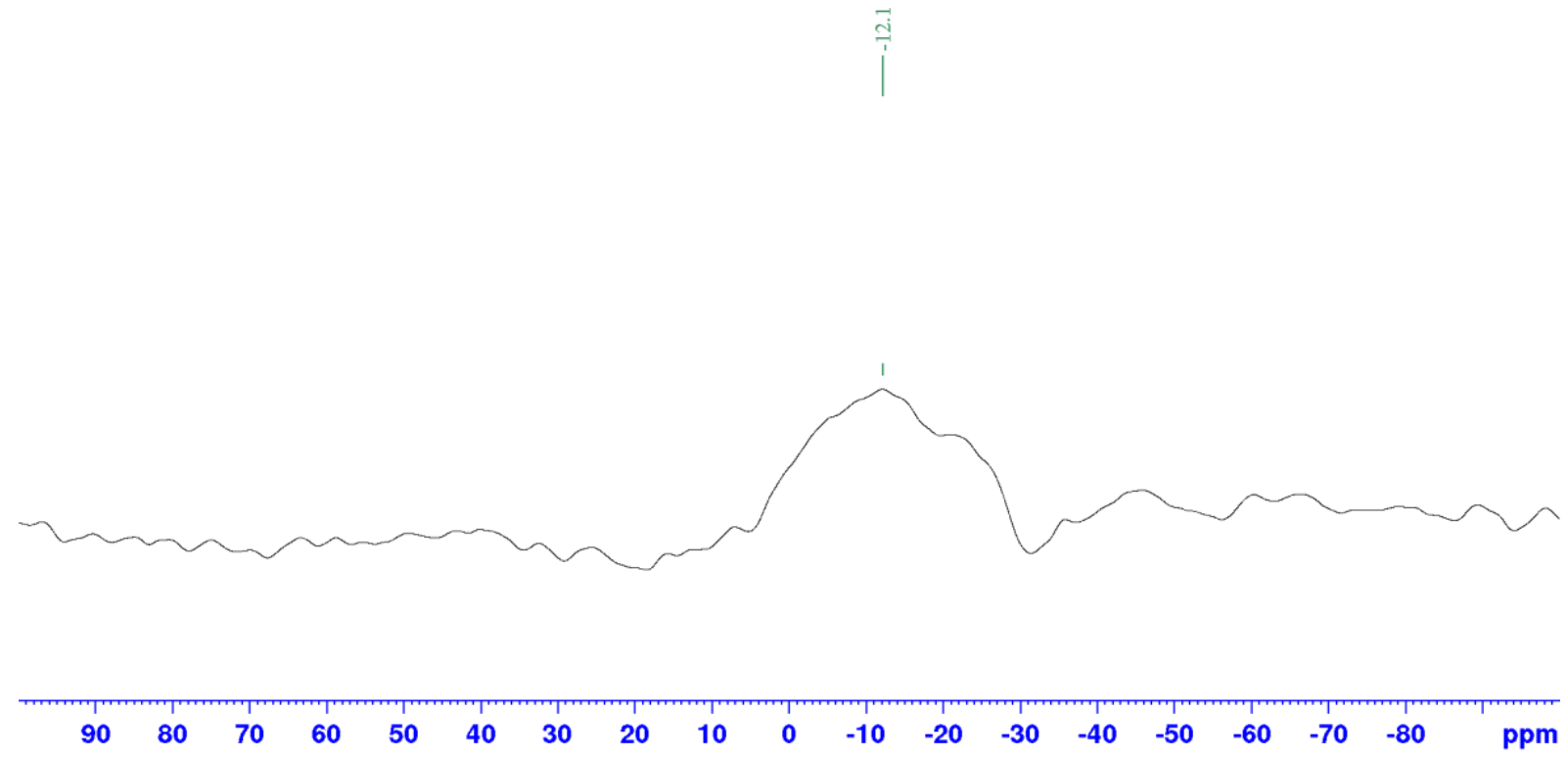

Figure S22 ${ }^{11} \mathrm{~B}\left\{{ }^{1} \mathrm{H}\right\}$-NMR spectrum $\left(96 \mathrm{MHz}, \mathrm{CDCl}_{3}\right)$ of $\mathbf{2 c}$.

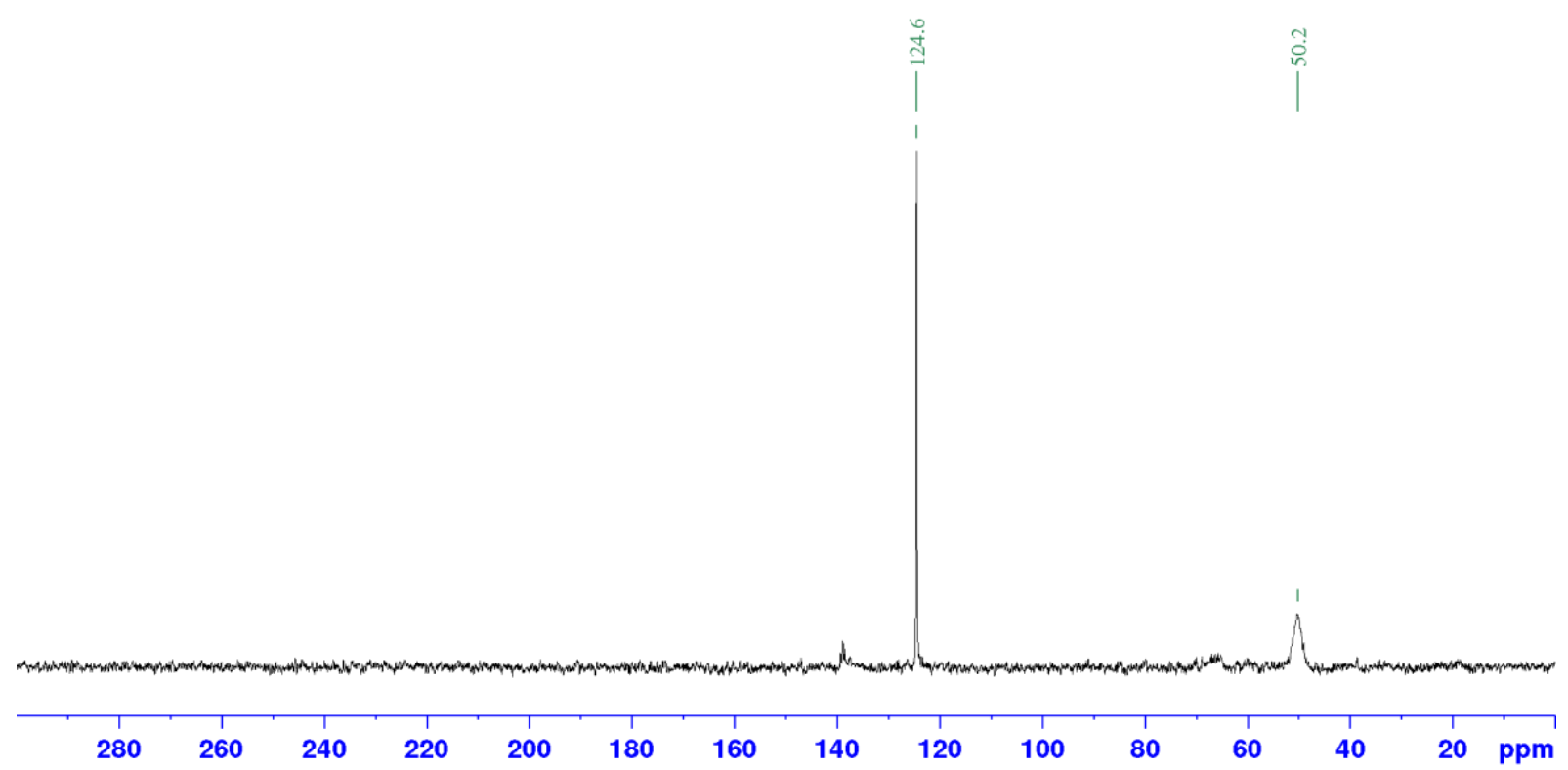

Figure $\mathbf{S 2 3}{ }^{31} \mathrm{P}\left\{{ }^{1} \mathrm{H}\right\}$-NMR spectrum $\left(122 \mathrm{MHz}, \mathrm{CDCl}_{3}\right)$ of $\mathbf{2 c}$. 


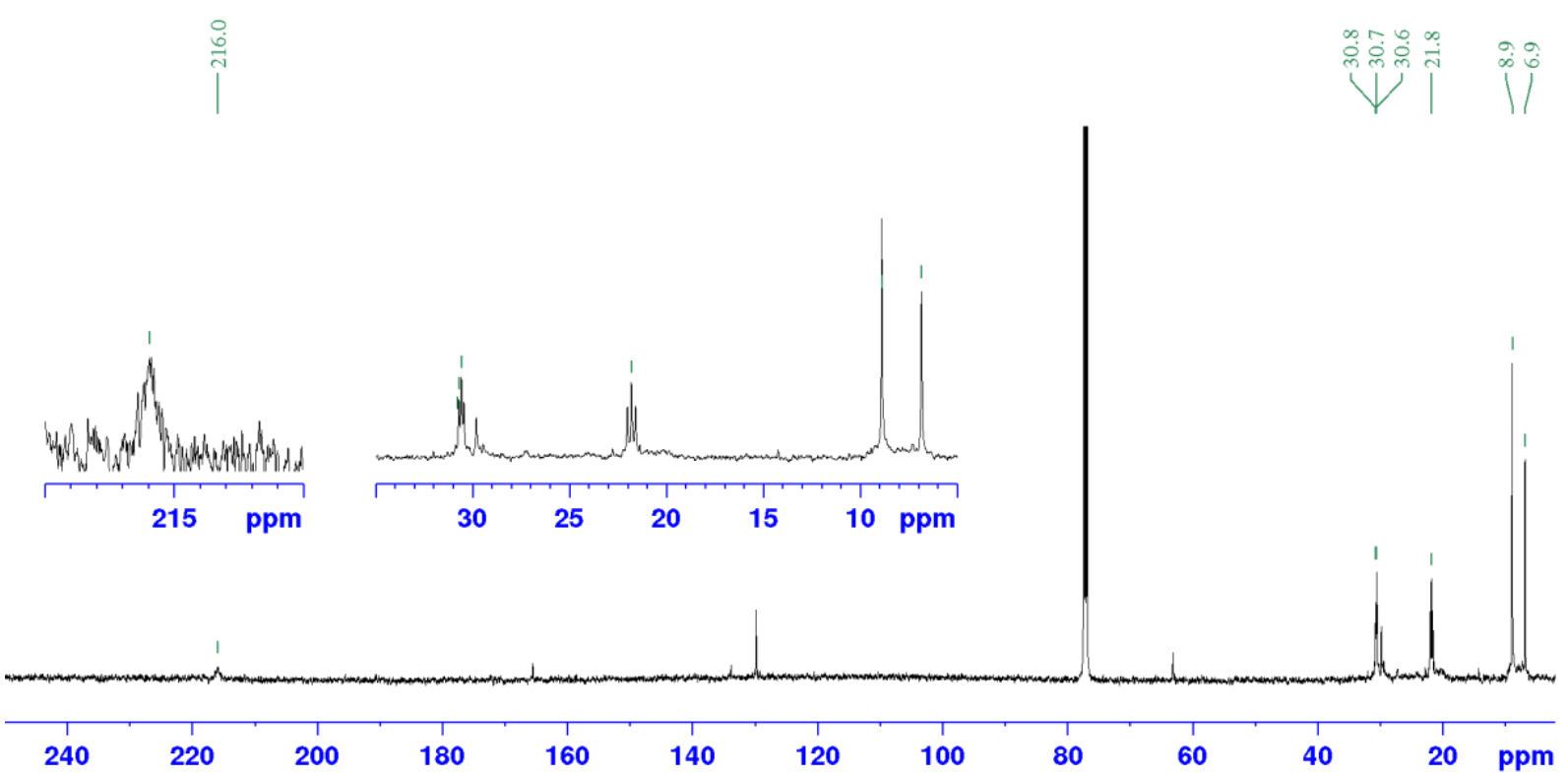

Figure $\mathbf{S 2 4}{ }^{13} \mathrm{C}\left\{{ }^{1} \mathrm{H}\right\}$-NMR spectrum $\left(126 \mathrm{MHz}, \mathrm{CDCl}_{3}\right)$ of $\mathbf{2 c}$.

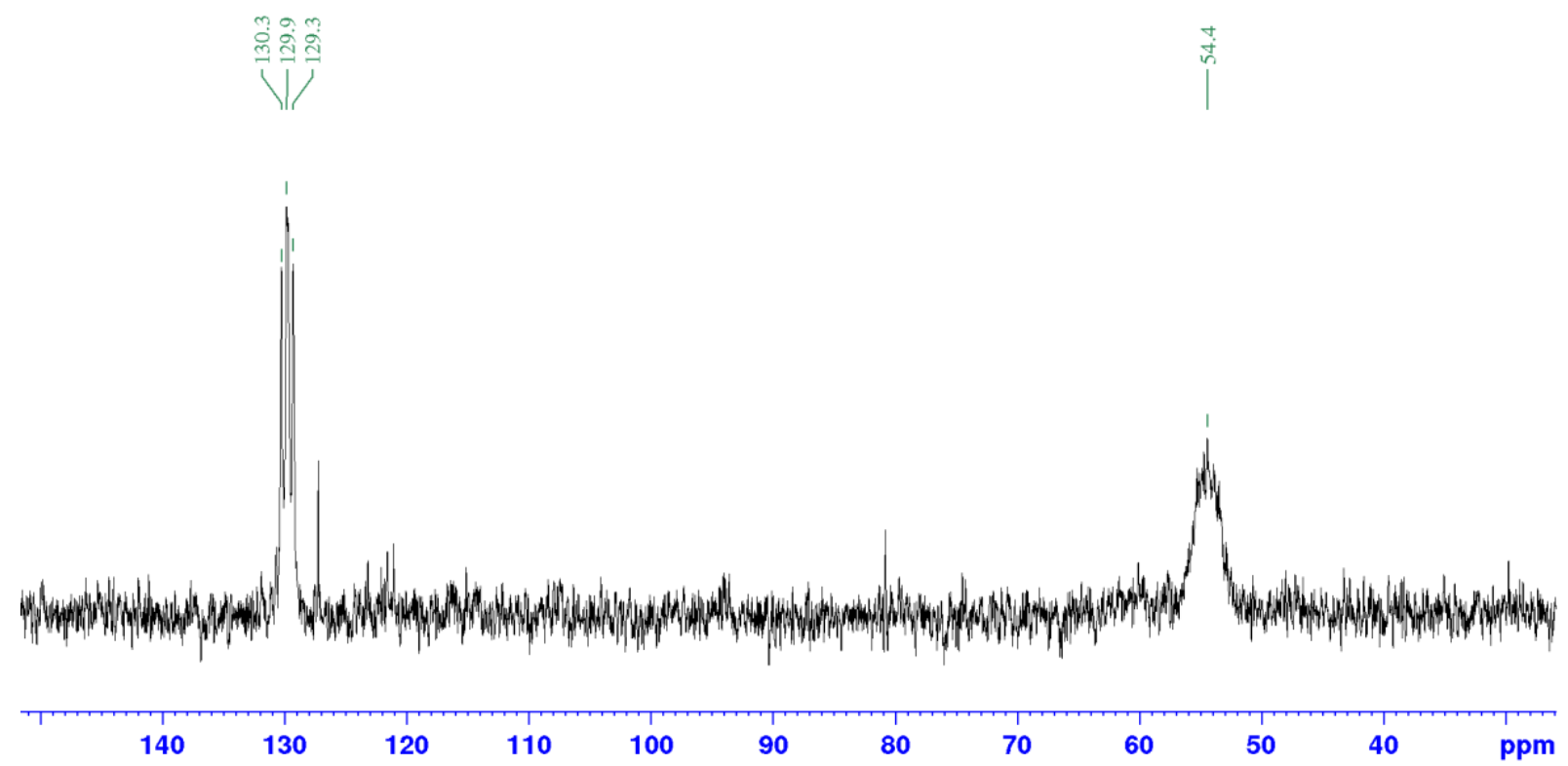

Figure $\mathbf{S 2 5}{ }^{31} \mathrm{P}\left\{{ }^{1} \mathrm{H}\right\}$ - NMR spectrum $\left(122 \mathrm{MHz}, \mathrm{CD}_{3} \mathrm{CN}\right)$ of $\mathbf{3 a}$. 


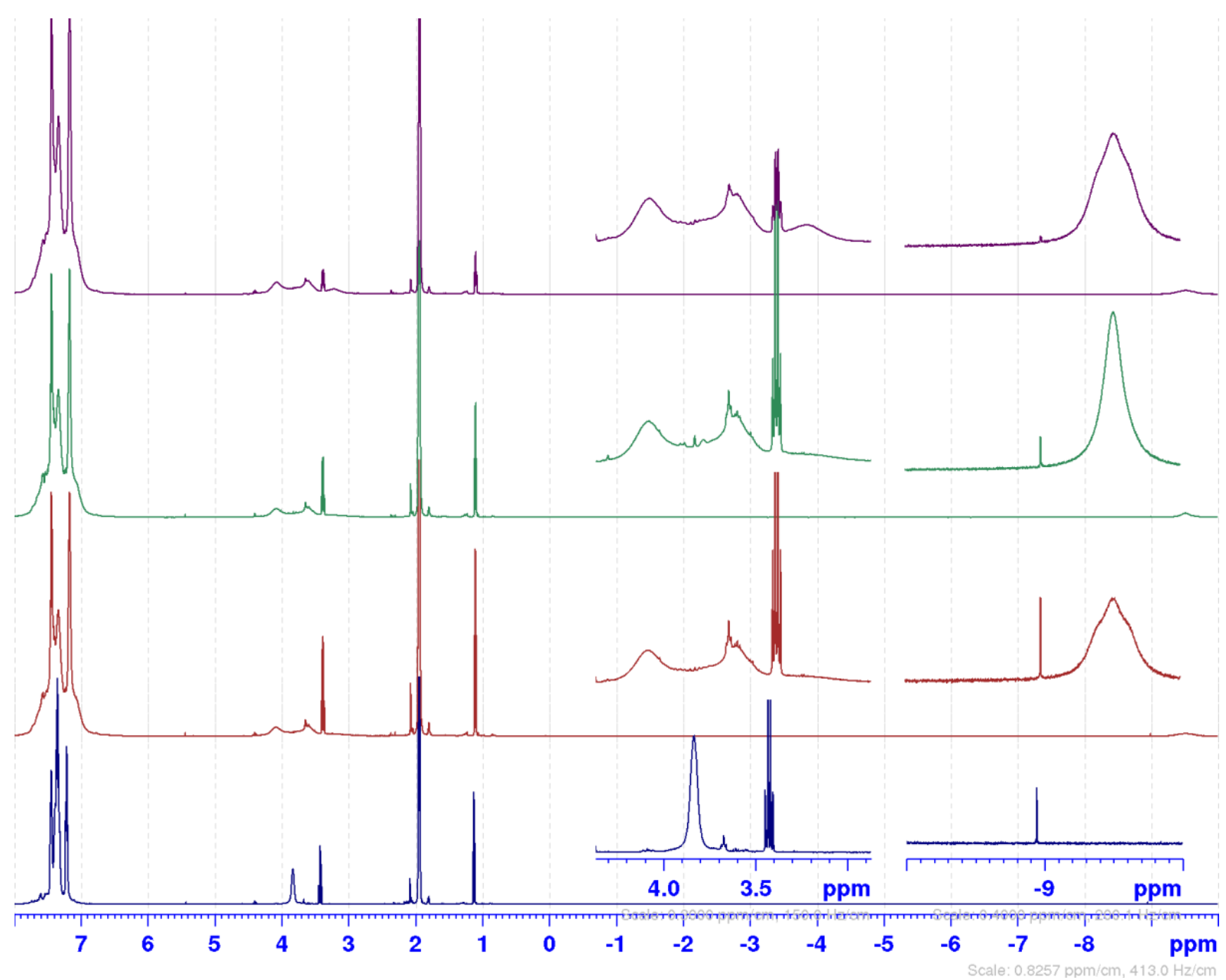

Figure S26 ${ }^{1} \mathrm{H}$ - NMR spectrum (blue) of $\mathbf{3 b}$ at $300 \mathrm{~K},{ }^{1} \mathrm{H}$ - NMR spectrum of $\mathbf{3 b}$ at $240 \mathrm{~K}$ (red), ${ }^{1} \mathrm{H}\left\{{ }^{11} \mathrm{~B}\right\}$ - NMR spectrum of $\mathbf{3 b}$ at $240 \mathrm{~K}$ (lilac) and ${ }^{1} \mathrm{H}\left\{{ }^{31} \mathrm{P}\right\}-\mathrm{NMR}$ spectrum of $\mathbf{3 b}$ at $240 \mathrm{~K}$ (green) (all: $500 \mathrm{MHz}, \mathrm{CD}_{3} \mathrm{CN}$ ).

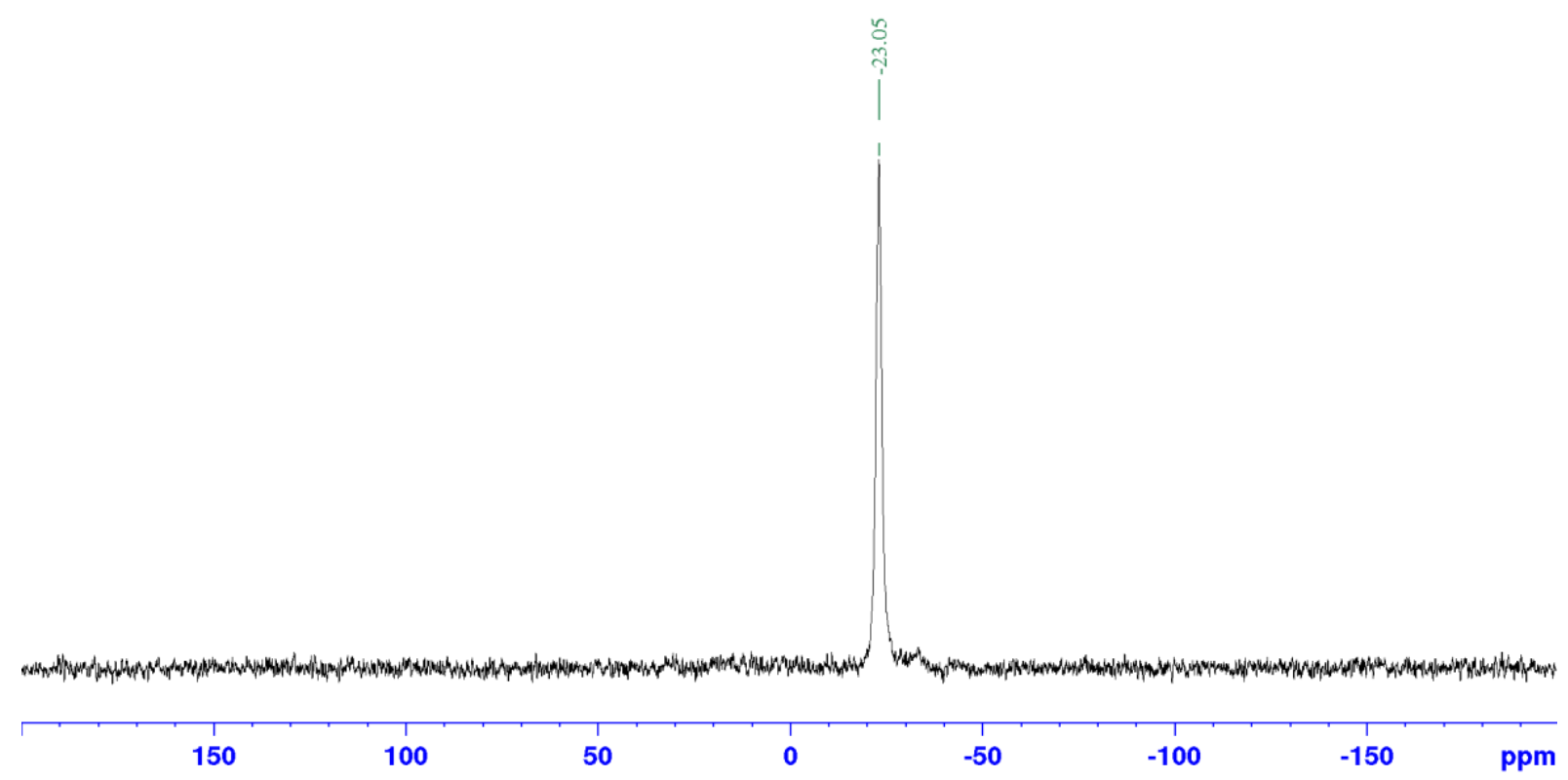

Figure $\mathbf{S 2 7}{ }^{11} \mathrm{~B}\left\{{ }^{1} \mathrm{H}\right\}-\mathrm{NMR}$ spectrum $\left(96 \mathrm{MHz}, \mathrm{CD}_{3} \mathrm{CN}\right)$ of $\mathbf{3 b}$. 


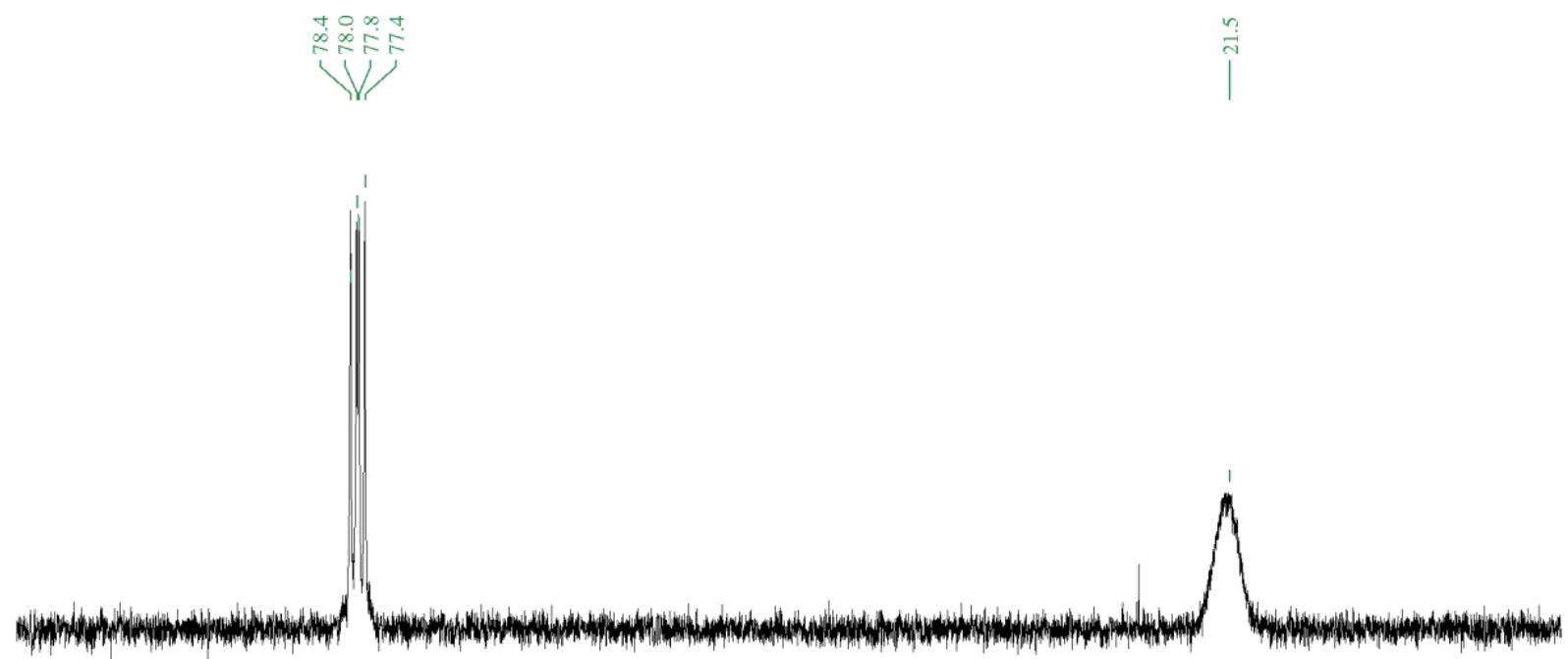

$\begin{array}{lllllllllllllllllll}95 & 90 & 85 & 80 & 75 & 70 & 65 & 60 & 55 & 50 & 45 & 40 & 35 & 30 & 25 & 20 & 15 & 10 & \mathrm{ppm}\end{array}$ Figure $\mathbf{S 2 8}{ }^{31} \mathrm{P}\left\{{ }^{1} \mathrm{H}\right\}$ - NMR spectrum $\left(122 \mathrm{MHz}, \mathrm{CD}_{2} \mathrm{Cl}_{2}\right)$ of $\mathbf{3 b}$.

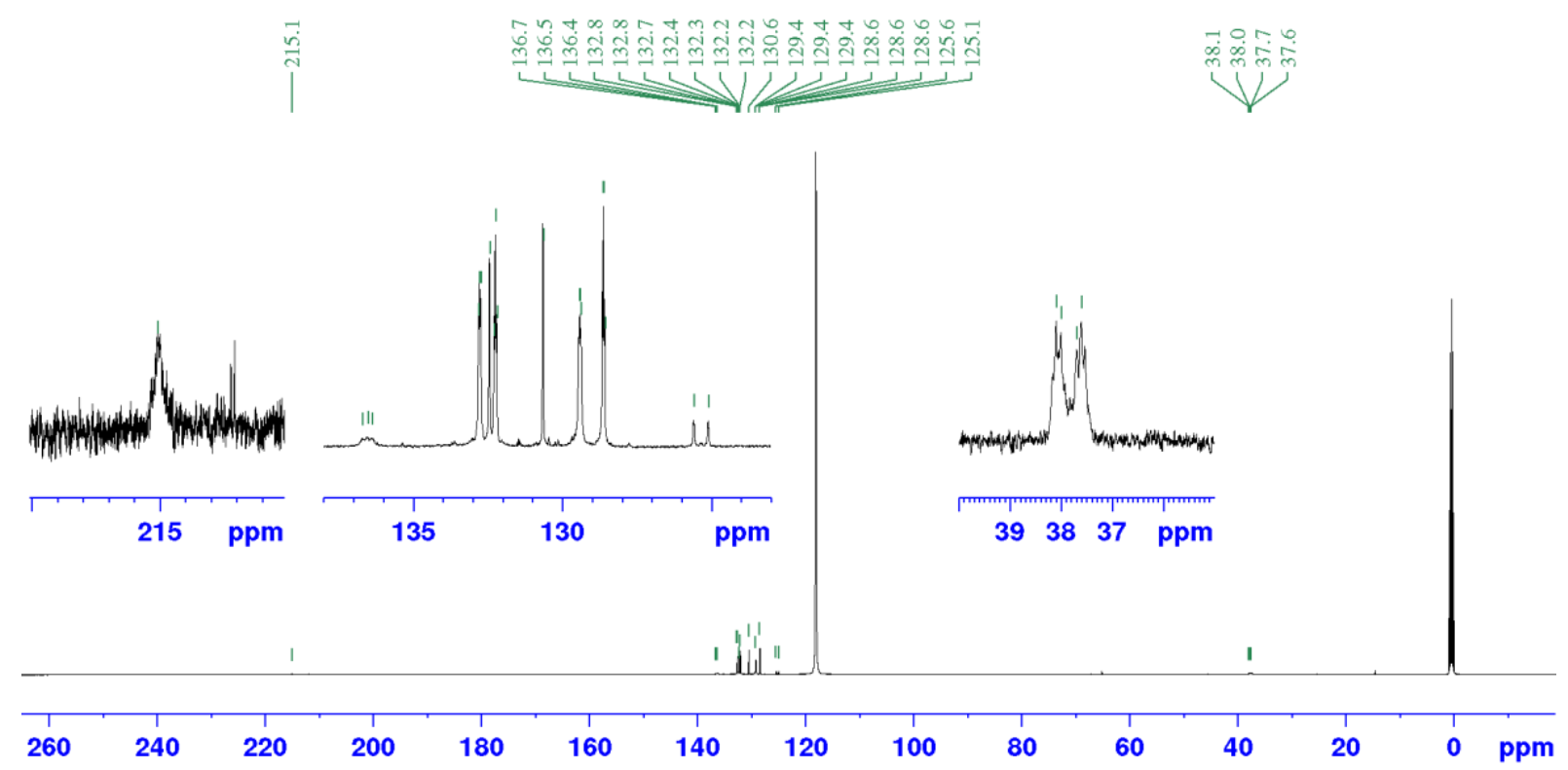

Figure $\mathbf{S 2 9}{ }^{13} \mathrm{C}$ - NMR spectrum (126 Mhz, $\left.\mathrm{CD}_{3} \mathrm{CN}\right)$ of $\mathbf{3 b}$. 


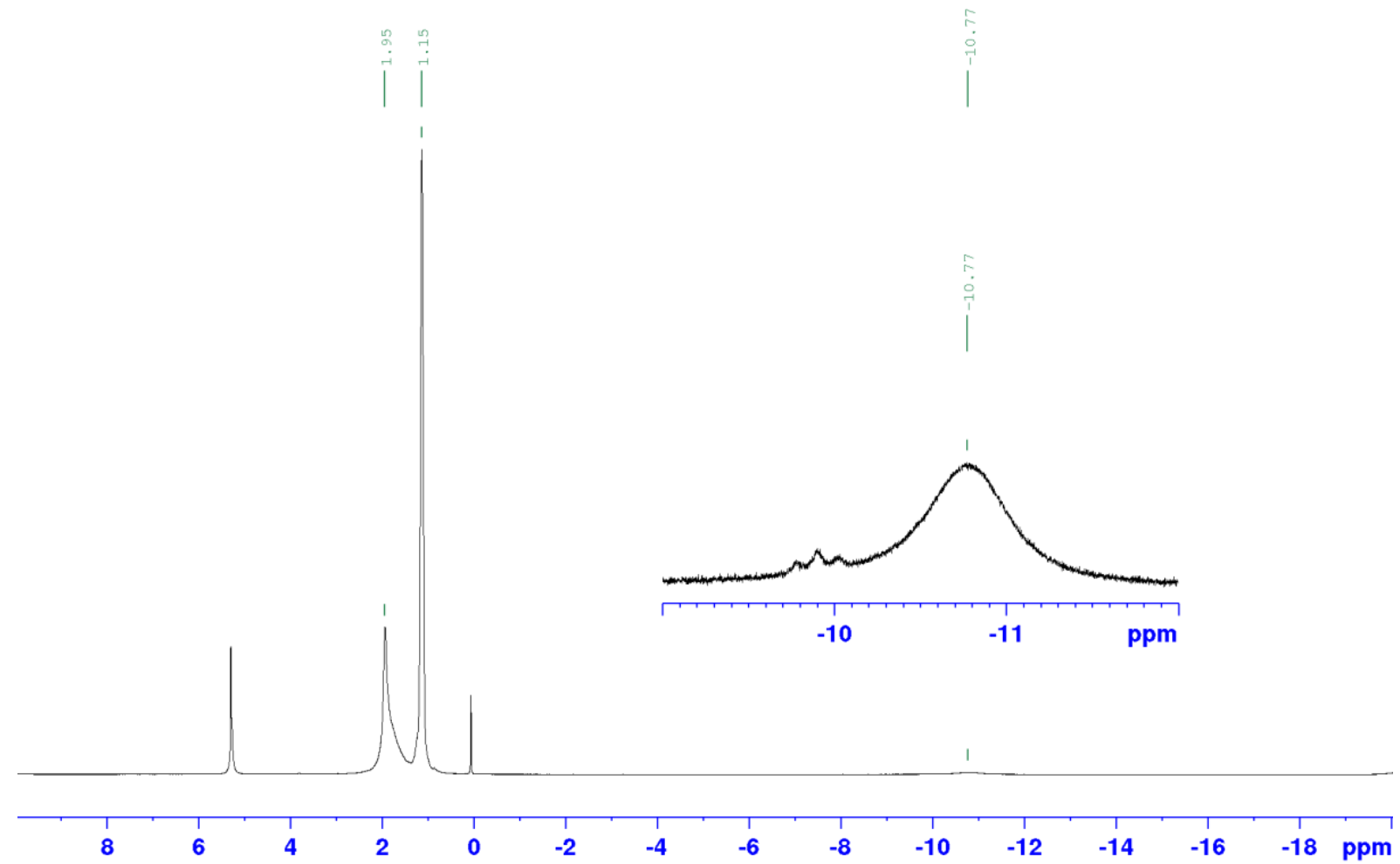

Figure $\mathbf{S 3 0}{ }^{1} \mathrm{H}-\mathrm{NMR}$ spectrum $\left(500 \mathrm{MHz}, \mathrm{CD}_{2} \mathrm{Cl}_{2}\right)$ of $\mathbf{3 c}$.

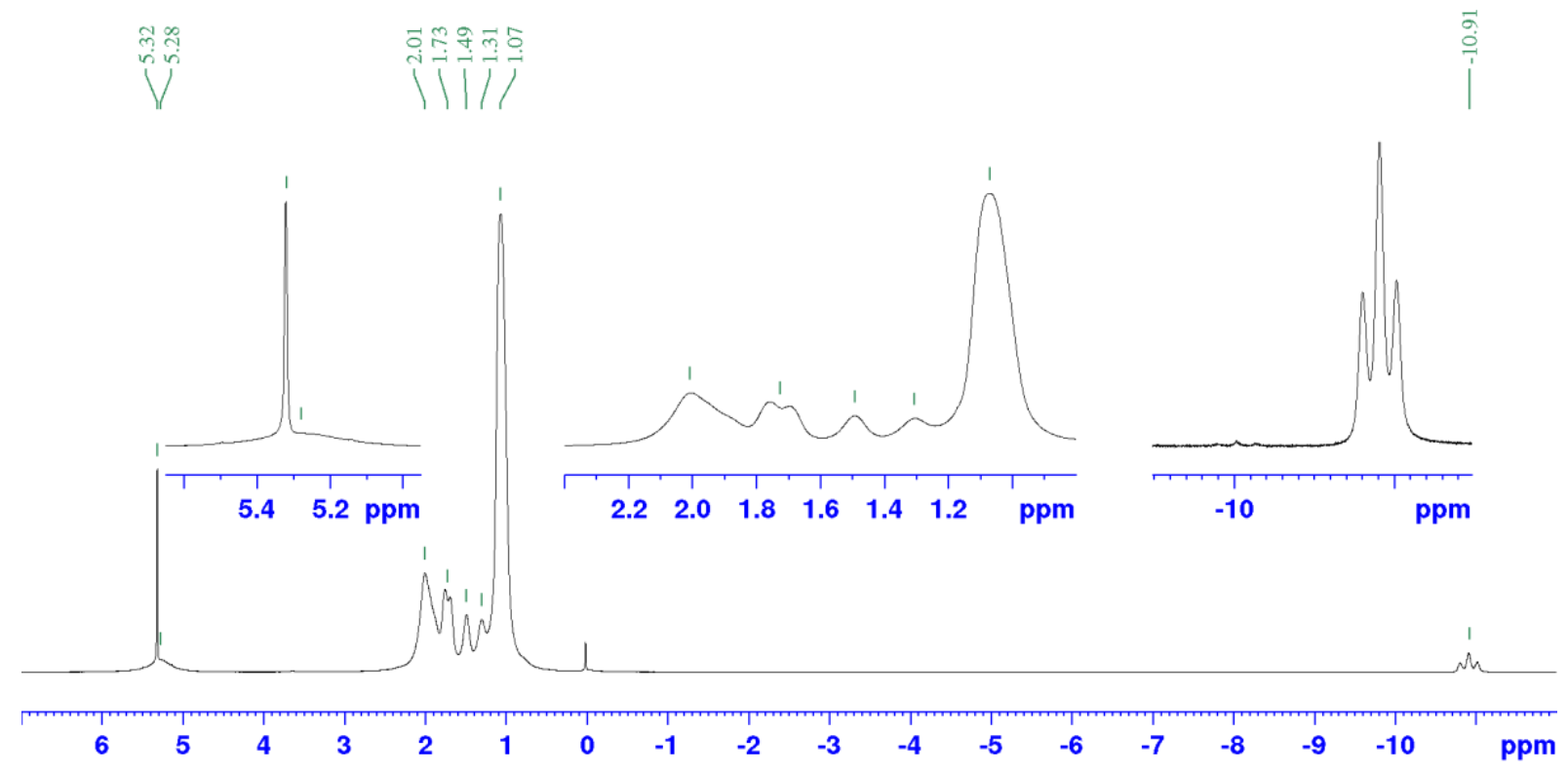

Figure S31 ${ }^{1} \mathrm{H}-\mathrm{NMR}$ spectrum $\left(500 \mathrm{MHz}, \mathrm{CD}_{2} \mathrm{Cl}_{2}\right)$ of $\mathbf{3 c} 213 \mathrm{~K}$. 


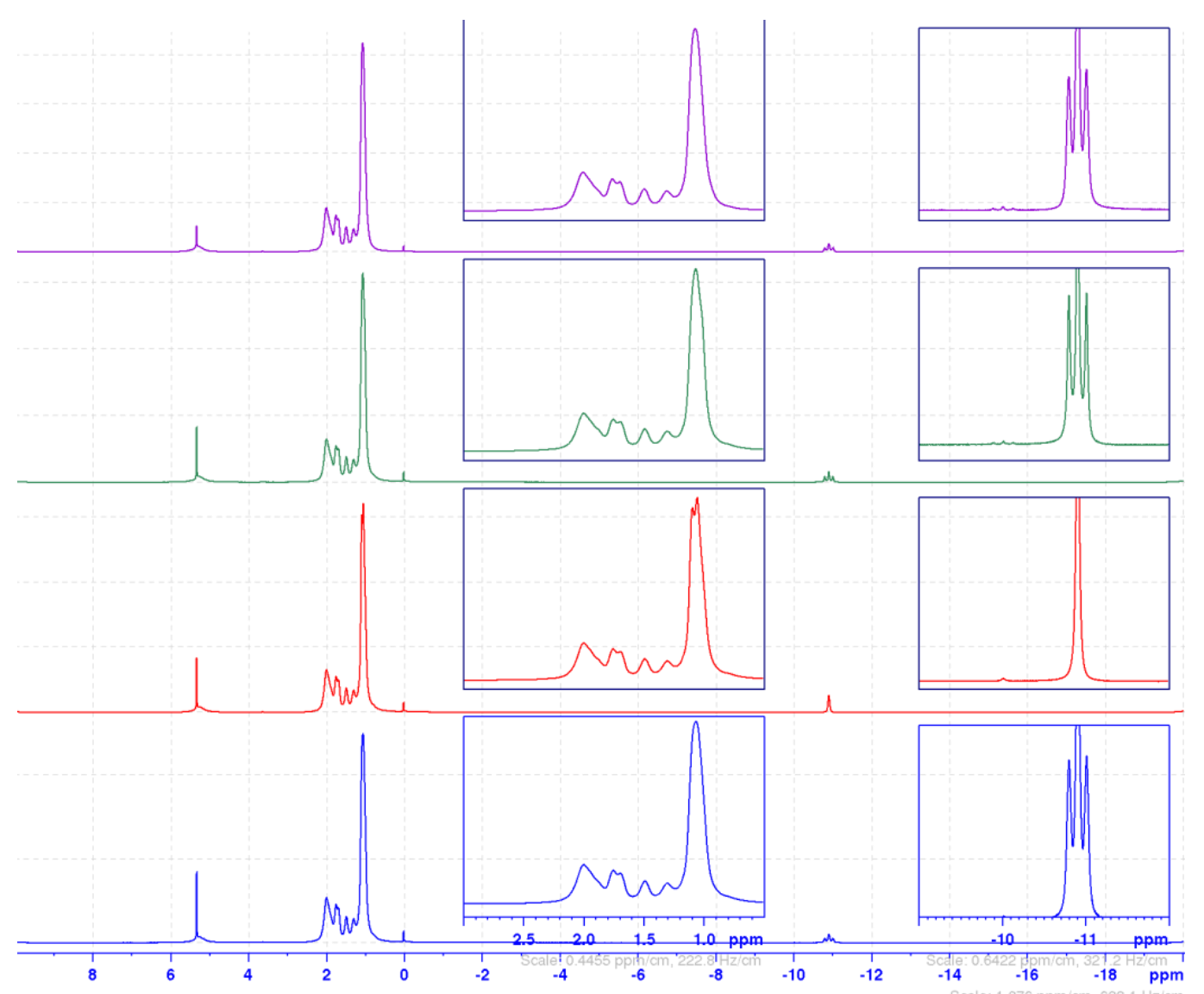

Figure S32 ${ }^{1} \mathrm{H}-\mathrm{NMR}$ spectrum (blue), ${ }^{1} \mathrm{H}\left\{{ }^{31} \mathrm{P}\right\}$-NMR spectra (green and red) and ${ }^{1} \mathrm{H}\left\{{ }^{11} \mathrm{~B}\right\}$-NMR (lilac) $\left(500 \mathrm{MHz}, \mathrm{CD}_{2} \mathrm{Cl}{ }_{2}\right.$, $\left.60^{\circ} \mathrm{C}\right)$ of $3 \mathbf{c}$.

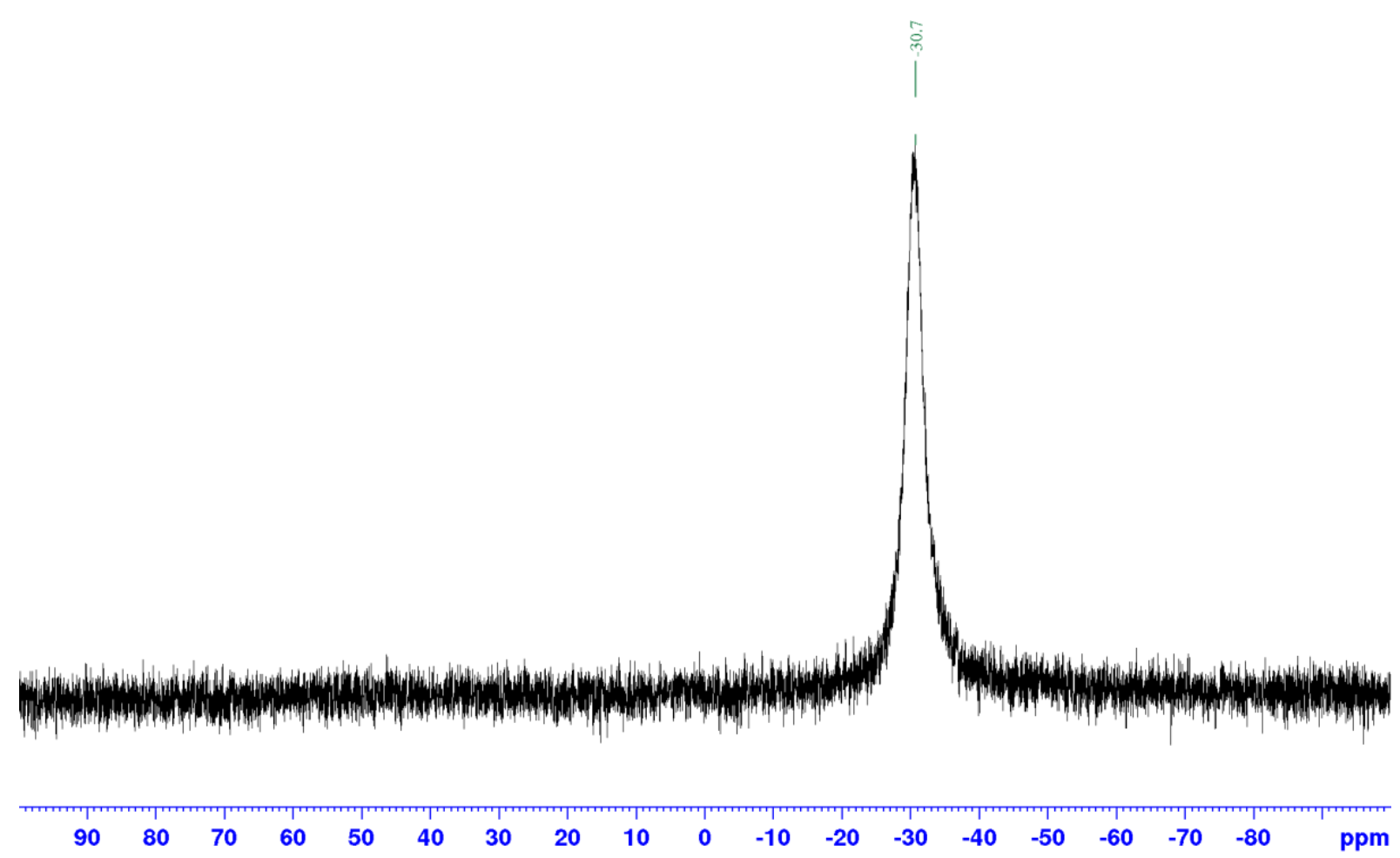

Figure $\mathbf{S 3 3}{ }^{11} \mathrm{~B}\left\{{ }^{1} \mathrm{H}\right\}$-NMR spectrum $\left(161 \mathrm{MHz}, \mathrm{CD}_{2} \mathrm{Cl}_{2},-60{ }^{\circ} \mathrm{C}\right)$ of $\mathbf{3 c}$. 


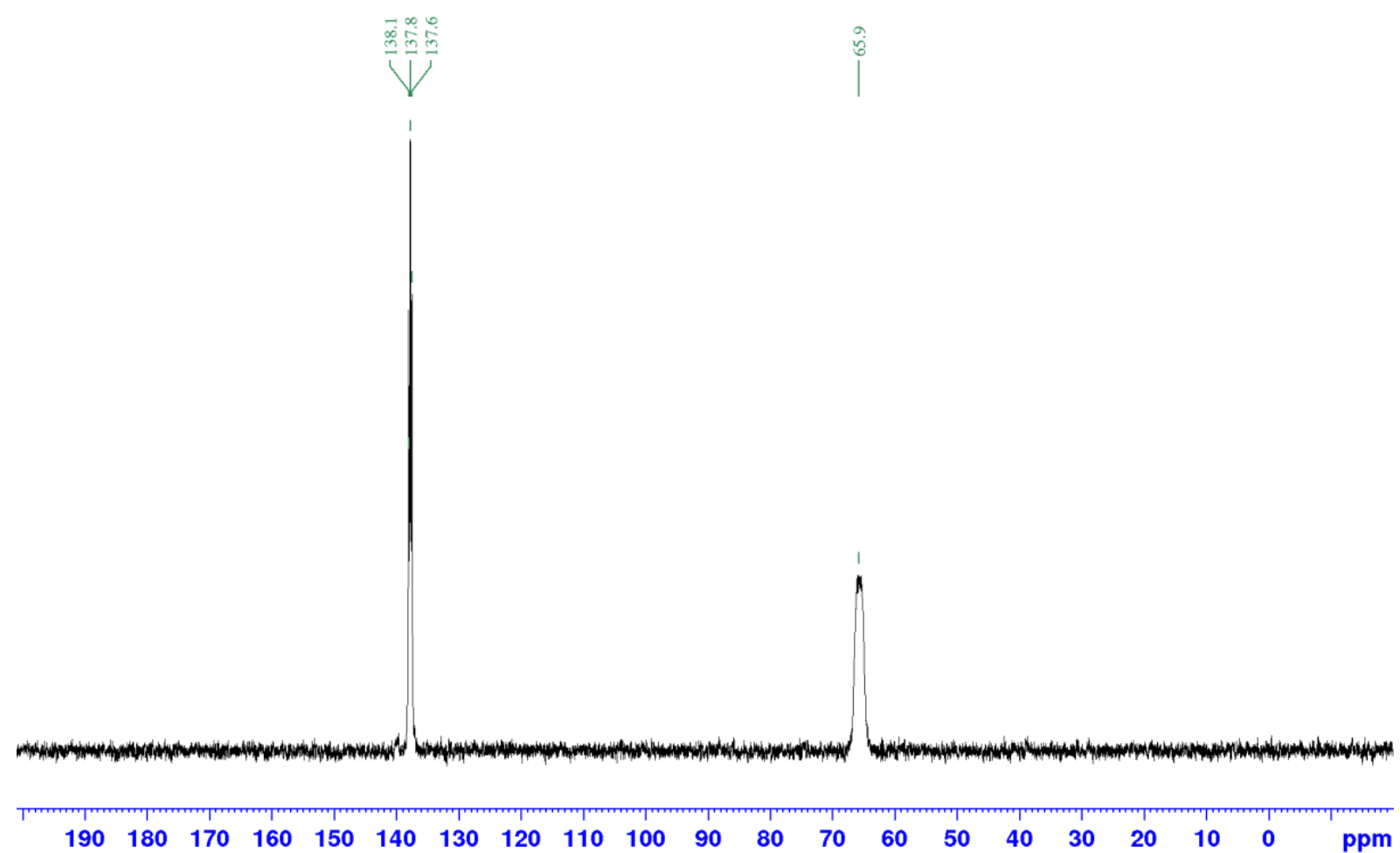

Figure $\mathbf{S 3 4}{ }^{31} \mathrm{P}\left\{{ }^{1} \mathrm{H}\right\}$-NMR spectrum (122 MHz, $\left.\mathrm{CD}_{2} \mathrm{Cl}_{2}\right)$ of $\mathbf{3 c}$.

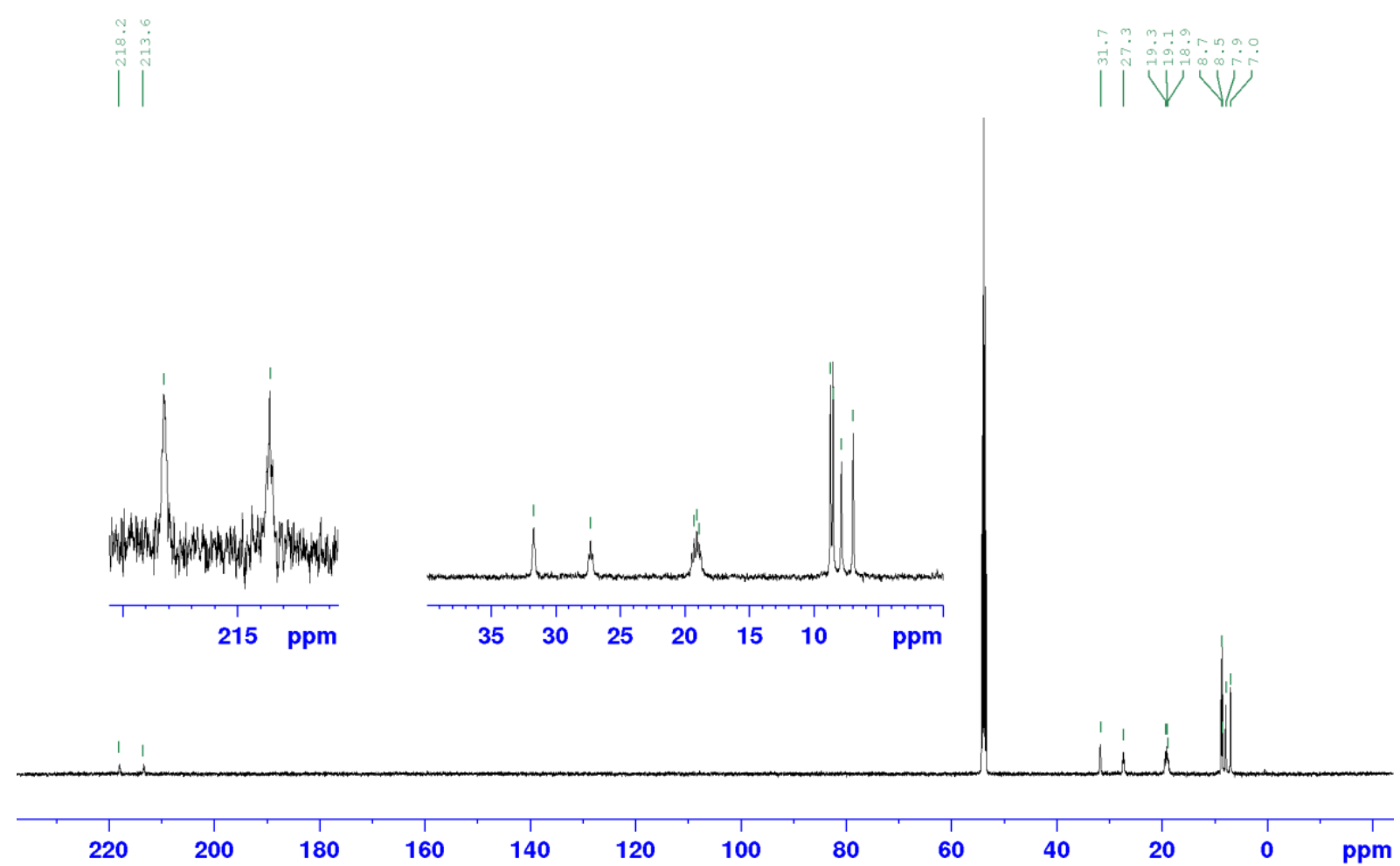

Figure $\mathbf{S 3 5}{ }^{13} \mathrm{C}\left\{{ }^{1} \mathrm{H}\right\}$-NMR spectrum $\left(125 \mathrm{MHz}, \mathrm{CD}_{2} \mathrm{Cl}_{2},-60{ }^{\circ} \mathrm{C}\right)$ of $\mathbf{3 c}$. 


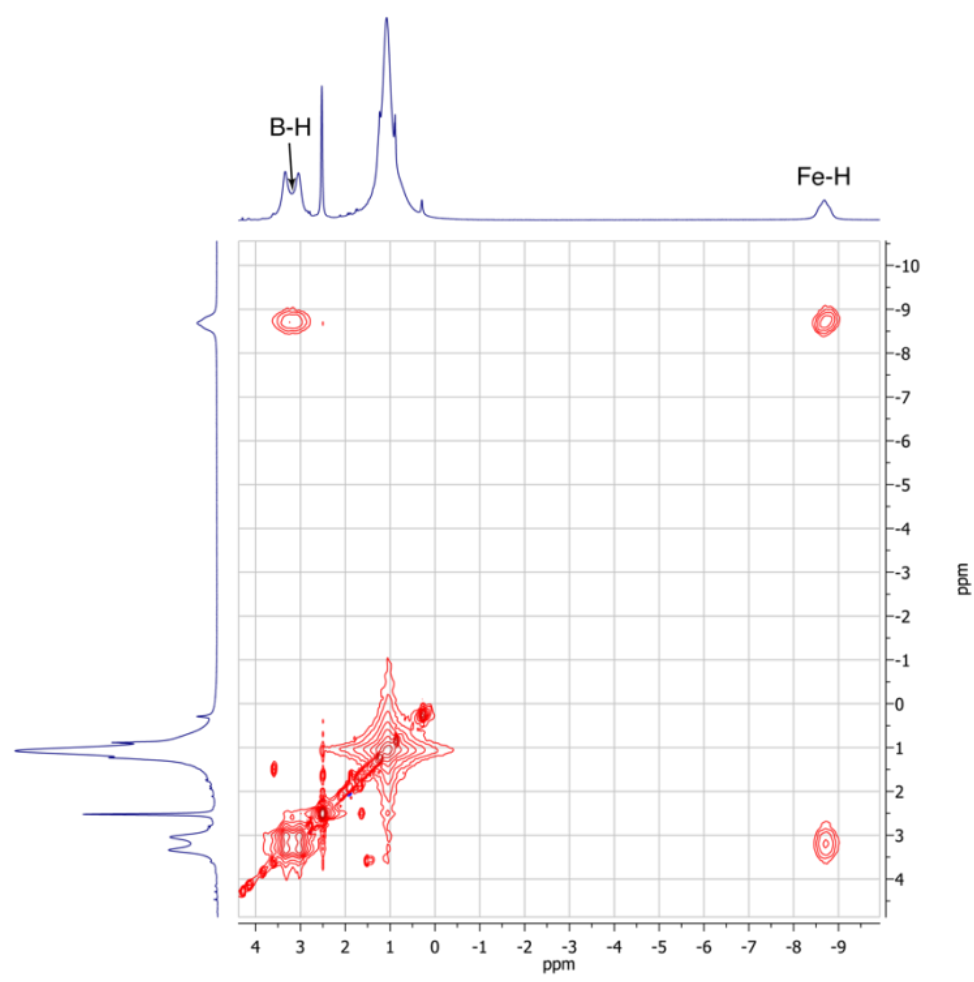

Figure S36 ${ }^{1} \mathrm{H}$ NOESY NMR spectrum of complex $\mathbf{5 b}$ right after the deprotonation in $\mathrm{C}_{6} \mathrm{D}_{6}\left(\mathbf{3 b}+\mathrm{KO}^{\mathrm{t}} \mathrm{Bu}\right)$, showing chemical exchange between the $\mathrm{Fe}-\mathrm{H}$ and the $\mathrm{B}-\mathrm{H}$ resonances.

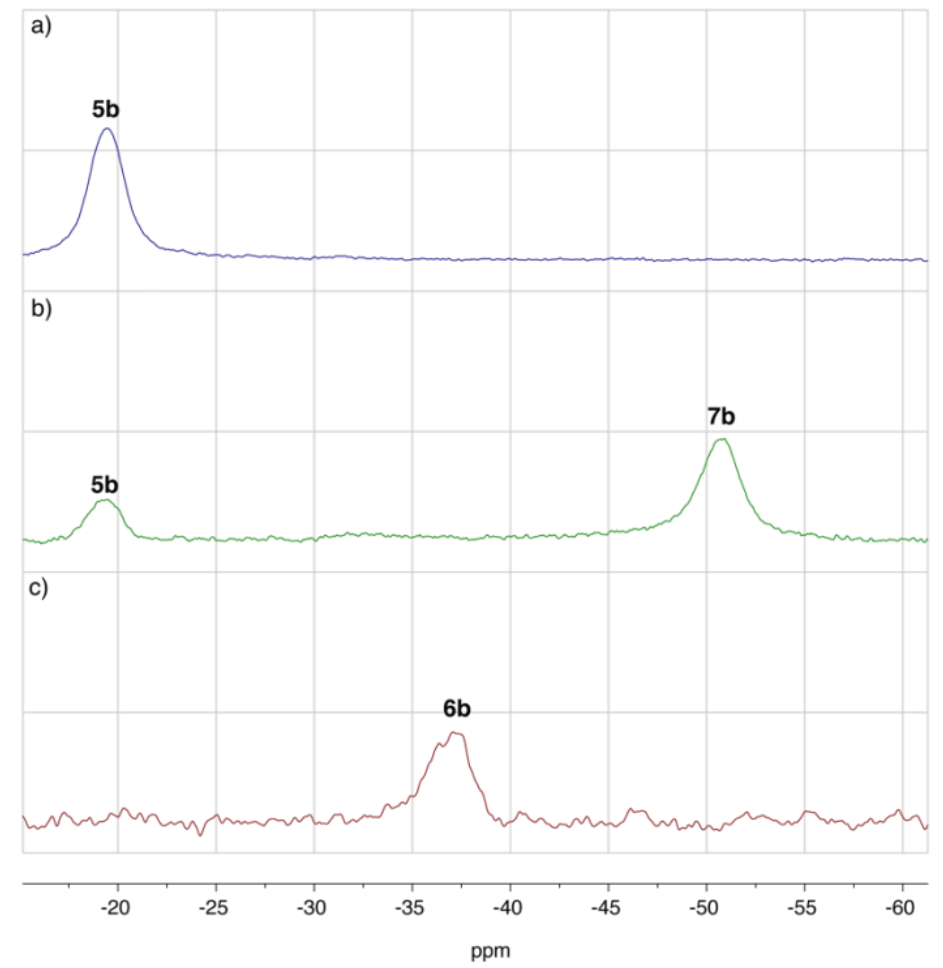

Figure S37 Examples of ${ }^{11} \mathrm{~B}\left\{{ }^{1} \mathrm{H}\right\}$ NMR spectra of $\mathbf{3 b}$ in the presence of base and $\left.\mathrm{Me}_{2} \mathrm{NH}_{-} \mathrm{BH}_{3}: \mathbf{a}\right) \mathbf{3 b}+1$ eq. $\mathrm{KO} \mathrm{Bu}^{t} \mathrm{Bu}$;) $\mathbf{3 b}+$ 10 eq. $\mathrm{KO}{ }^{t} \mathrm{Bu}$; c) $3 \mathbf{b}+1.4$ eq. $\mathrm{LiN}\left(\mathrm{SiMe}_{3}\right)_{2}+4$ eq. $\mathrm{Me}_{2} \mathrm{NH}-\mathrm{BH}_{3}$. Please note that almost identical results were obtained with different base $\left(\mathrm{KO}^{t} \mathrm{Bu}, \mathrm{LiN}\left(\mathrm{SiMe}_{3}\right)_{2}\right.$ and $\left.\mathrm{KH}\right)$ and with different amounts of $\mathrm{Me}_{2} \mathrm{NH}-\mathrm{BH}_{3}$. 


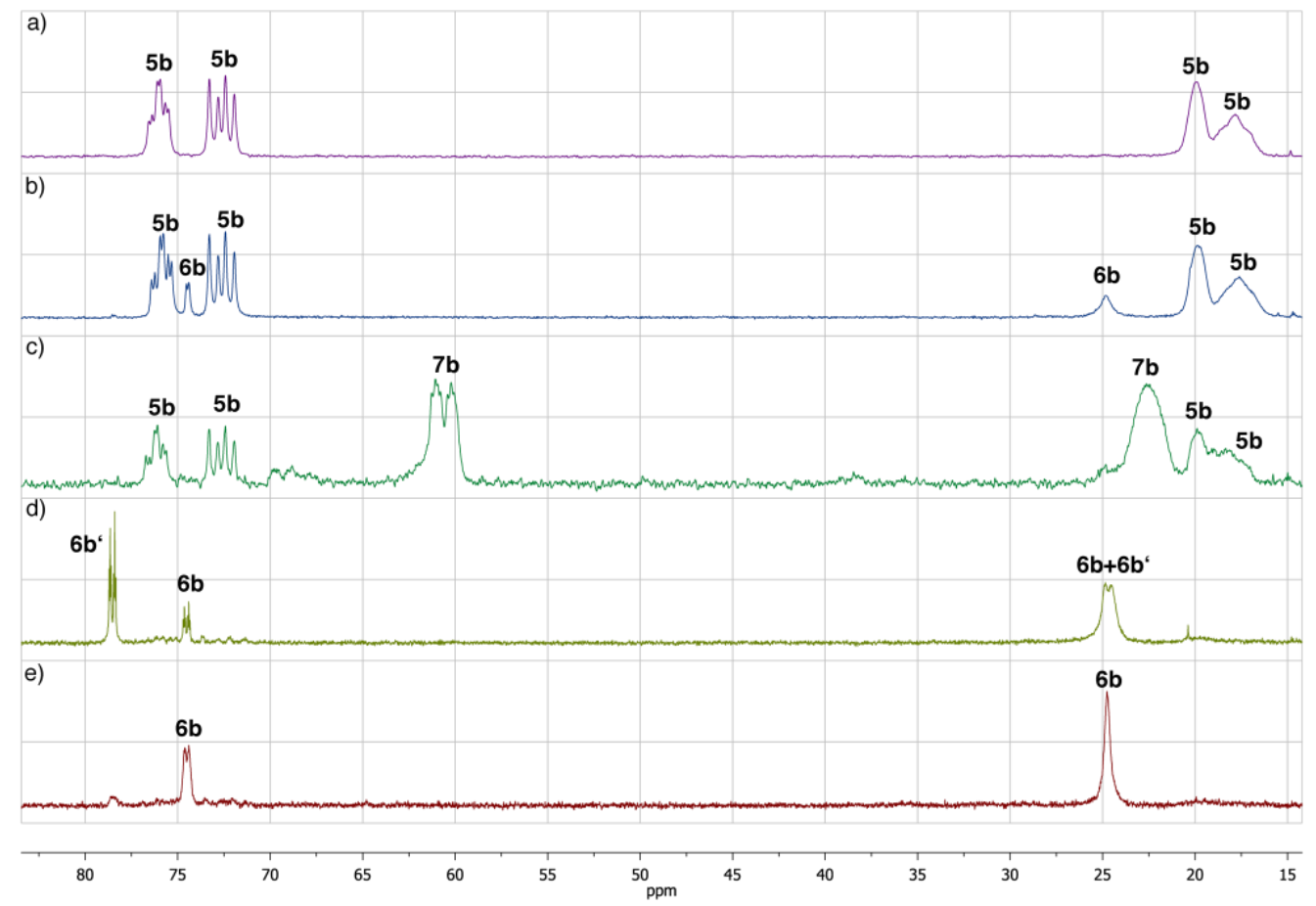

Figure S38 Examples of ${ }^{31} \mathrm{P}\left\{{ }^{1} \mathrm{H}\right\}$ NMR spectra of the reaction of $\mathbf{3 b}$ with base and $\mathrm{Me}_{2} \mathrm{NH}-\mathrm{BH}$ : a) $\mathbf{3 b}+1$ eq. $\mathrm{KO} \mathrm{B}^{\mathrm{B}} \mathrm{B}$; $\left.\mathbf{b}\right)$ reaction mixture from spectrum a after 2 days; c) $3 \mathbf{b}+10$ eq. $\mathrm{KO}{ }^{t} \mathrm{Bu}$; d) reaction mixture from spectrum a and b+2 eq. $\mathrm{Me}_{2} \mathrm{NH}-$ $\mathrm{BH}_{3}$ e) reaction mixture from spectrum $\mathrm{c}+8$ eq. $\mathrm{Me}_{2} \mathrm{NH}-\mathrm{BH}_{3}$.

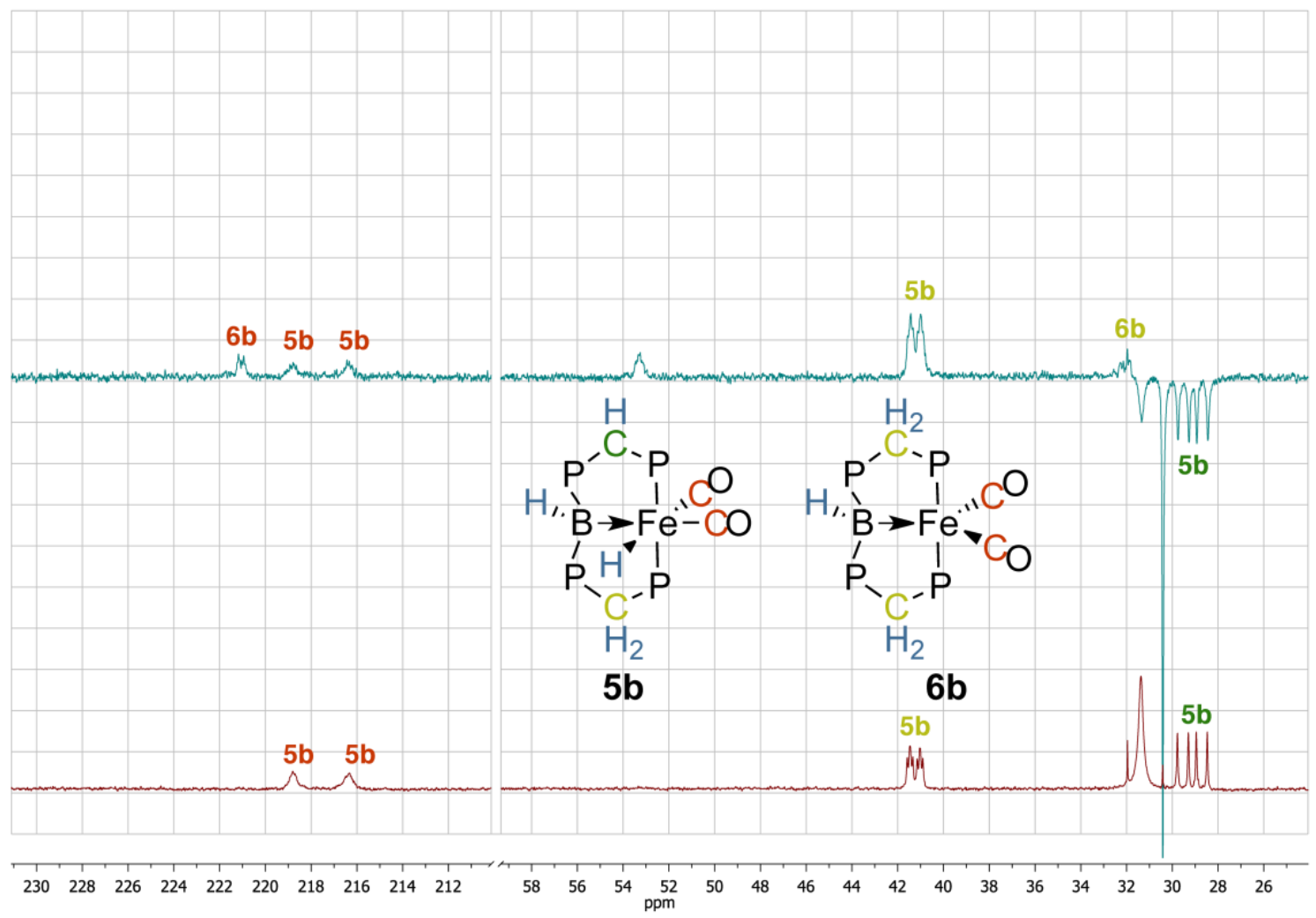

Figure S39 Indicative regions of the ${ }^{13} \mathrm{C}\left\{{ }^{1} \mathrm{H}\right\}$ NMR spectrum of $\mathbf{5 b}$ (obtained by the reaction of $\mathbf{3 b}+1$ eq. $\mathrm{KO}^{t} \mathrm{Bu}$ ) after addition of base (bottom) and of the ${ }^{13} \mathrm{C}$ APT NMR spectrum after two days (top). 


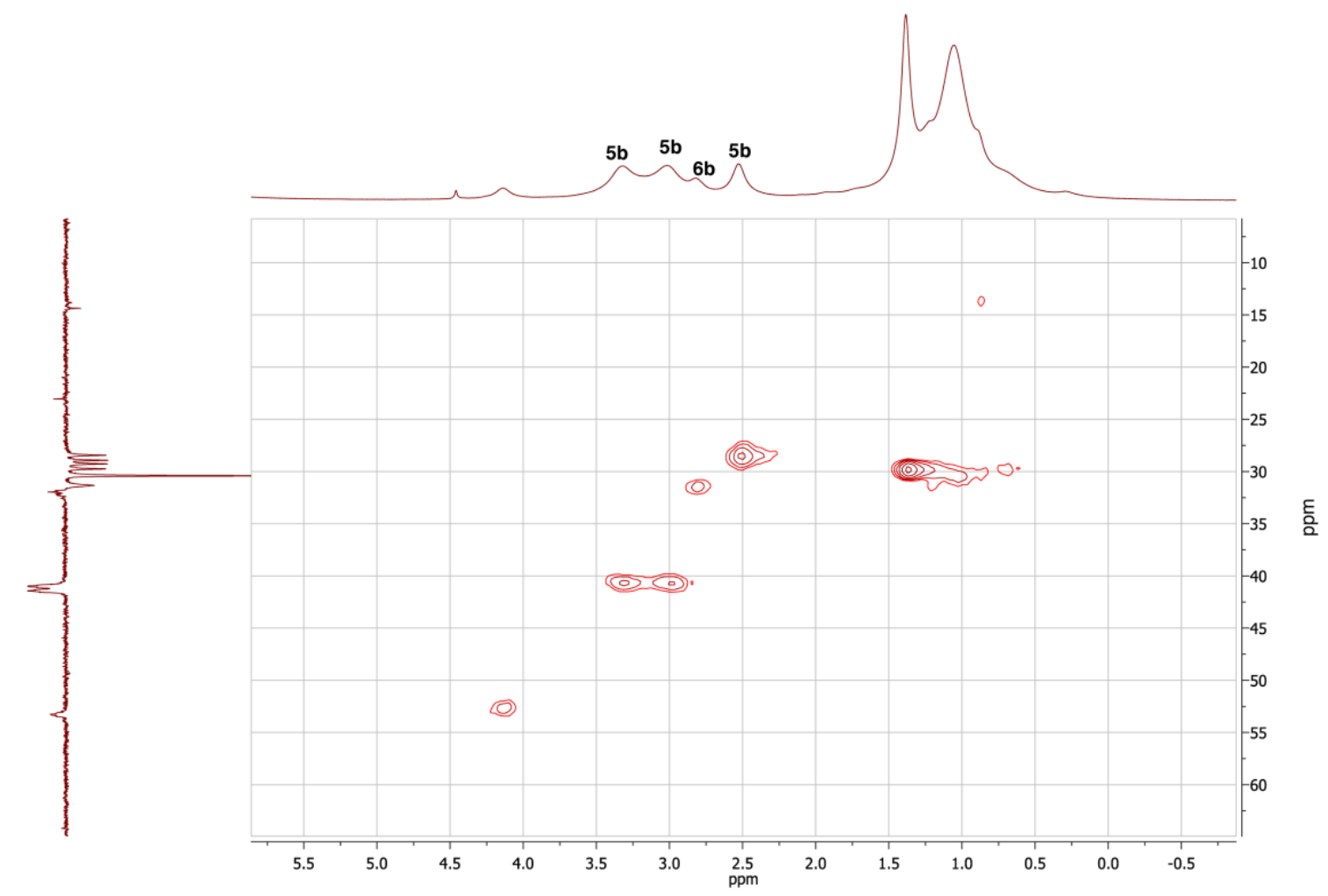

Figure $\mathbf{S 4 0}{ }^{1} \mathrm{H},{ }^{13} \mathrm{C}$ HMQC NMR spectrum of $\mathbf{5 b}$ and $\mathbf{6 b}$ showing the $\mathrm{P}_{-} \mathrm{CH}_{\mathrm{n}}-\mathrm{P}$ region.

\section{Variable Temperature NMR Measurements}

The rate constants of the intramolecular exchange reactions in complex $\mathbf{3 a - 3} \mathbf{b}$ were determined by line shape analysis of the resonance corresponding the hydrido ligand in the ${ }^{1} \mathrm{H}$ and ${ }^{1} \mathrm{H}\left\{{ }^{31} \mathrm{P}\right\}$ NMR spectra. The line broadening due to chemical exchange $\Delta v_{1 / 2}$ of these resonances was obtained as the difference of the half height width $\left(v_{1 / 2}\right)$ of the hydrido resonance and a reference peak at each temperature. As reference the $\mathrm{CH}_{3}$-resonance of silicon grease was used. The approximate rate constants can be calculated using equation 1.

$$
k_{e x}=1 / 2 \pi\left(\vartheta_{1 / 2}-\vartheta_{R e f}\right)
$$

The presence of an intramolecular exchange was further confirmed by ${ }^{1} \mathrm{H}$ NOESY NMR spectra at different temperatures as well as by the concentration independence of the rate constant. Table 1 summarizes the kinetic data. 
Table S1 Kinetic data for the intramolecular exchange in $\mathbf{3 a - 3 b}$, obtained by line shape analysis.

\begin{tabular}{crrr}
\hline $\mathrm{T} / \mathrm{K}$ & $k_{e x} / \mathrm{s}^{-1}(\mathbf{3 a})$ & $k_{e x} / \mathrm{s}^{-1}(\mathbf{3 b})$ & $k_{e x} / \mathrm{s}^{-1}(\mathbf{3 c})$ \\
\hline 330 & 32.26 & - & - \\
320 & 17.83 & - & - \\
310 & 12.00 & - & $*$ \\
300 & 8.77 & $*$ & 434.90 \\
290 & 6.74 & - & 261.32 \\
285 & - & 2687.67 & - \\
280 & - & - & 157.53 \\
270 & - & 1192.40 & - \\
260 & - & - & 28.36 \\
255 & - & 423.83 & - \\
240 & - & 124.10 & 11.84 \\
\hline
\end{tabular}

* denotes coalescence 


\section{X-Ray Crystallography}

The single crystal X-ray diffraction data for the structural analysis were collected using graphite-monochromated Mo-K $\alpha$-radiation $\left(\lambda \mathrm{MoK}_{\alpha}=0.71073\right)$ on the imaging plate detector system STOE IPDS2T or on the pixel detector system Bruker Quest D8. The structures were solved with the Olex2 or APEX (v2016.1) software by direct methods with SHELXT and refined against $\mathrm{F}^{2}$ by full-matrix-least-square techniques using SHELXL. ${ }^{4-8}$ Based on the crystal descriptions, numerical absorption corrections were applied ${ }^{8}$ Crystallographic data for 1b, 2c, 3b and 3c was deposited at Cambridge Crystallographic Data Centre (CCDC 1884368 -1884371) and can be obtained free of charge via www.ccdc.cam.ac.uk/. 
Table S2 X-Ray crystallographic data for $\mathbf{1 b}, \mathbf{2 c}, \mathbf{3 b}$ and $\mathbf{3 c}$.

\begin{tabular}{|c|c|c|c|c|}
\hline Compound & $\mathbf{1 b}$ & $2 \mathrm{c}$ & $3 \mathrm{~b} \cdot 2^{1} /{ }_{2} \mathrm{CH}_{2} \mathrm{Cl}_{2}$ & $3 c$ \\
\hline Empirical formula & $\mathrm{C}_{48} \mathrm{H}_{44} \mathrm{BBrN}_{2} \mathrm{P}_{4}$ & $\mathrm{C}_{19} \mathrm{H}_{43} \mathrm{BBrFeN}_{2} \mathrm{O}_{3} \mathrm{P}_{4}$ & $\mathrm{C}_{52} \mathrm{H}_{46} \mathrm{BBrFeO}_{2} \mathrm{P}_{4} \cdot 2^{1} /{ }_{2} \mathrm{CH}_{2} \mathrm{Cl}_{2}$ & $\mathrm{C}_{18} \mathrm{H}_{44} \mathrm{BBrFeN}_{2} \mathrm{O}_{2} \mathrm{P}_{4}$ \\
\hline$F w / g \cdot \mathrm{mol}^{-1}$ & 863.45 & 618.00 & 1185.65 & 591.00 \\
\hline$T / \mathrm{K}$ & $100(2)$ & $100(2)$ & $100(2)$ & $100(2)$ \\
\hline Crystal system & Triclinic & Orthorhombic & Triclinic & Monoclinic \\
\hline Space group & $P \overline{1}$ & $P_{n a 2}$ & $P \overline{1}$ & $P 2_{1} / c$ \\
\hline$a / \AA$ & $10.551(2)$ & $14.623(3)$ & $11.496(2)$ & $12.7253(9)$ \\
\hline$b / \AA$ & $12.062(2)$ & $15.131(3)$ & $12.374(3)$ & $10.5434(7)$ \\
\hline$c / \AA$ & $18.905(4)$ & $12.874(3)$ & $20.858(4)$ & $21.3482(14)$ \\
\hline$\alpha /^{\circ}$ & $82.63(3)$ & 90.00 & $97.02(3)$ & 90 \\
\hline$\beta /{ }^{\circ}$ & $81.72(3)$ & 90.00 & $94.81(3)$ & $102.389(2)$ \\
\hline$\gamma /{ }^{\circ}$ & $66.18(3)$ & 90.00 & $111.71(3)$ & 90 \\
\hline$V / \AA^{3}$ & $2171.5(9)$ & $2848.7(10)$ & $2708.9(11)$ & $2797.5(3)$ \\
\hline$Z$ & 2 & 4 & 2 & 4 \\
\hline$\rho_{\text {calc }} / \mathrm{g} \cdot \mathrm{cm}^{-3}$ & 1.321 & 1.441 & 1.454 & 1.403 \\
\hline$\mu\left(\mathrm{MoK}_{\alpha}\right) / \mathrm{mm}^{-1}$ & 1.130 & 2.179 & 1.421 & 2.213 \\
\hline $\mathrm{F}(000)$ & 892 & 1284 & 1210 & 1232 \\
\hline $2 \theta$ range $^{\circ}$ & $3.70-53.43$ & $5.00-52.74$ & $3.59-51.70$ & $4.53-48.45$ \\
\hline Reflections measured & 19598 & 16613 & 20677 & 32306 \\
\hline Independent reflections & $9145\left(R_{\mathrm{Int}}=0.0560\right)$ & $5811\left(R_{\mathrm{Int}}=0.1411\right)$ & $10351\left(R_{\text {Int }}=0.1114\right)$ & $4483\left(R_{\mathrm{Int}}=0.0727\right)$ \\
\hline Ind. reflections $(I>2 \sigma(I))$ & 8107 & 5249 & 6931 & 3529 \\
\hline Parameters/Restraints & $513 / 0$ & $293 / 1$ & 711 & $317 / 12$ \\
\hline Flack parameter & - & $0.031(15)$ & - & - \\
\hline$R_{1}(I>2 \sigma(I))$ & 0.0391 & 0.0649 & 0.0864 & 0.0433 \\
\hline$w R_{2}($ all data $)$ & 0.1242 & 0.1786 & 0.2538 & 0.1147 \\
\hline GooF (all data) & 1.109 & 1.078 & 1.011 & 1.031 \\
\hline Max. peak/hole/e $\cdot \AA^{-3}$ & $0.543 /-1.154$ & $0.606 /-1.061$ & $1.035 /-0.891$ & $0.688 /-0.584$ \\
\hline $\mathrm{CCDC}$ & 1884369 & 1884368 & 1884371 & 1884370 \\
\hline
\end{tabular}




\section{DFT Calculations}

\section{Computational Details}

All reported structures were optimized by the density functional theory (DFT) ${ }^{9}$ with the M06L functiona ${ }^{10}$ with basis sets I (BSI, lanl2 $\mathrm{dz}^{11}$ for metal atom and 6-31G $(\mathrm{d}, \mathrm{p})$ for nonmetal atoms) in the gas phase. Frequency analysis calculations of optimized structures were performed at the same level of theory (M06-L/BSI) to characterize the structures to be minima (no imaginary frequency) or transition states (one imaginary frequency). Intrinsic reaction coordinate (IRC) calculations were performed to confirm the connection between two correct minima for a transition state. Based on the M06-L/BSI optimized geometries, the energy results were further refined by calculating the single point energy at the M06-L/BSII level of theory (BSII designates $\mathrm{SDD}^{12}$ for metal atom and $6-311++\mathrm{G}^{* * 13}$ for nonmetal atoms). The bulky solvation effect of benzene $(\varepsilon=7.4)$ was simulated by SMD $^{12}$ continuum solvent mode at the M06-L/BSII level of theory. The standard Gibbs free energy in solution is calculated by equation (1).

$$
G_{(\mathrm{sol})}^{\circ}=G_{\mathrm{gas}}+\Delta G_{\mathrm{solv}}+R T \ln (24.5)
$$

Where the $G_{\text {gas }}$ is the gas-phase free energy, $G_{\text {solv }}$ is the solvation free energy. The final term accounts for the free energy change from an ideal gas of $1 \mathrm{~atm}(24.5 \mathrm{~L}, 298.15 \mathrm{~K})$ to $1 \mathrm{M}$ solution. All natural bond orbital (NBO) analysis were performed using the NBO 3.0 package. ${ }^{14}$ All the calculations were performed with the Gaussian 09 program. ${ }^{15}$ The $3 \mathrm{D}$ optimized structures were displayed by the CYLview visualization program. ${ }^{16}$

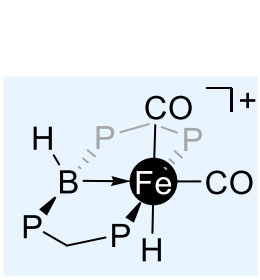

$3 \mathbf{b}$

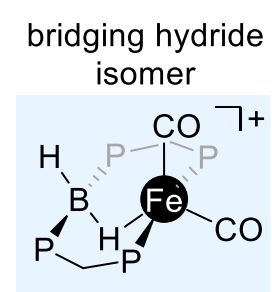

$3 b_{\text {iso1 }}$
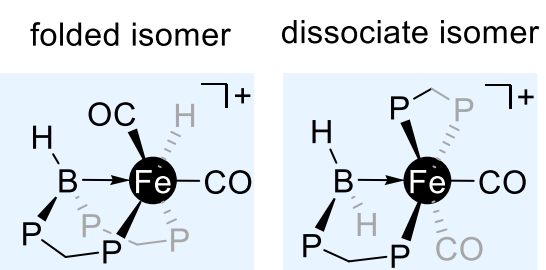

$3 b_{\text {iso2 }}$

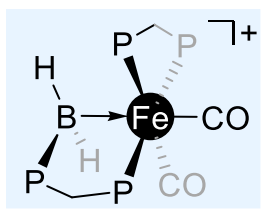

$3 b_{\text {iso3 }}$

Figure S41 Possible isomers for complex $\mathbf{3 b}$. 
$\Delta G / \Delta H \mathrm{kcal} / \mathrm{mol}$

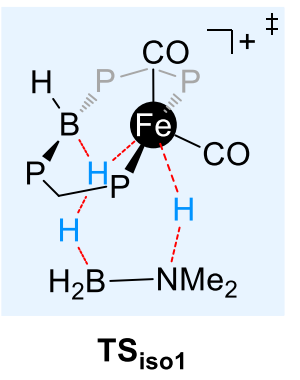

49.8/35.6

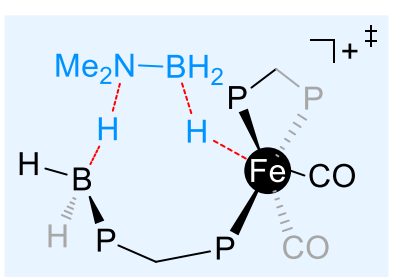

$\mathrm{TS}_{\text {iso4 }}$

$57.2 / 43.1$

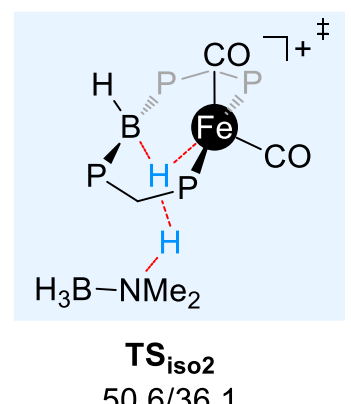

$50.6 / 36.1$

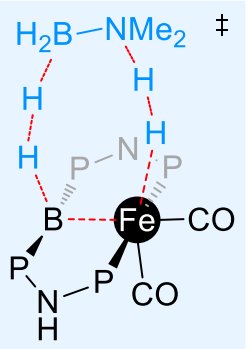

$\mathrm{TS}_{\text {iso5 }}$

$60.3 / 47.2$

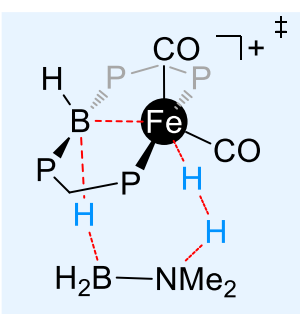

$\mathrm{TS}_{\text {iso3 }}$

$57.2 / 42.7$

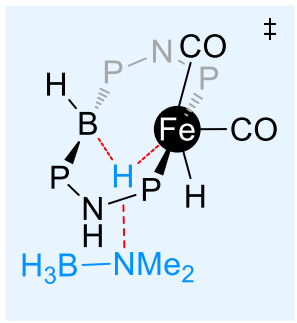

TS $_{\text {iso6 }}$

$51.8 / 37.5$

Figure $\mathbf{S 4 2}$ Possible transition states for the dehydrogenation of $\mathrm{NHMe}_{2} \mathrm{BH}_{3}$ by complex $\mathbf{3 b}\left(\mathbf{T S}_{\text {iso1-4) }}\right.$ ) and other similar catalytic species (TS iso5-6). $_{\text {- }}$ ).

\section{$\Delta G / \Delta H \mathrm{kcal} / \mathrm{mol}$}

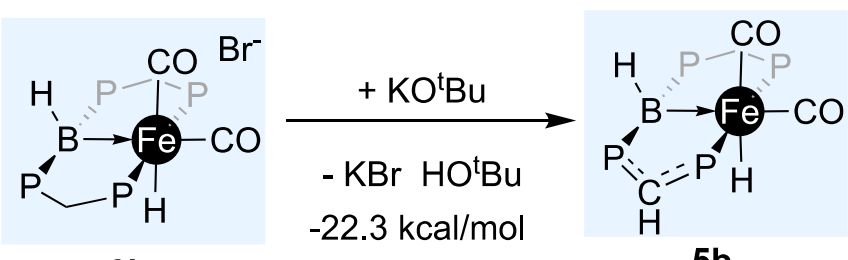

3b

$0.0 / 0.0$
$-18.8 /-8.9$

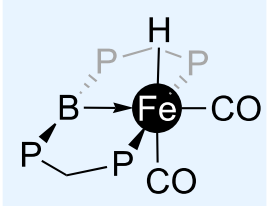

5 b'

7.1/18.1

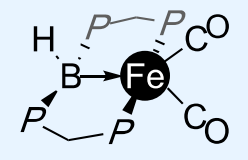

6b

$-22.2 /-8.5$

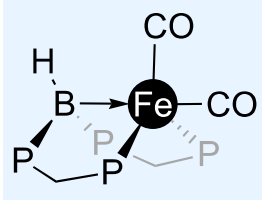

6b'

$-22.3 /-12.8$

Figure $\mathbf{S 4 3}$ Deprotonation of complex $3 \mathbf{b}$ to complex $\mathbf{5 b}, \mathbf{6 b}$ and possible isomers.

The Cartesian coordinates (xyz) for all optimized structures.

3b'

Fe $\quad-1.05994200 \quad 0.79338900 \quad-0.51558000$

$\begin{array}{llll}\mathrm{P} & 0.05253400 & 2.16534200 & 0.79070300\end{array}$ 


\begin{tabular}{|c|c|c|c|}
\hline$P$ & -2.19331200 & -1.02255000 & -1.04602000 \\
\hline $\mathrm{P}$ & 0.34384800 & -2.14153000 & 0.05100900 \\
\hline $\mathrm{P}$ & 2.30224700 & 0.42711800 & -0.18109000 \\
\hline $\mathrm{O}$ & -0.30978100 & 1.85515600 & -3.14047100 \\
\hline $\mathrm{O}$ & -3.54485500 & 2.29023200 & -0.09513600 \\
\hline $\mathrm{C}$ & -0.60808900 & 1.44686300 & -2.09126100 \\
\hline $\mathrm{C}$ & -0.72317000 & 4.86522100 & 0.72045900 \\
\hline $\mathrm{H}$ & -1.41814000 & 4.61236200 & 1.51783000 \\
\hline $\mathrm{C}$ & -0.00893000 & -2.27826000 & 1.82154800 \\
\hline $\mathrm{C}$ & 0.17449100 & 6.49234400 & -0.82092500 \\
\hline $\mathrm{H}$ & 0.18247500 & 7.50065000 & -1.22412800 \\
\hline $\mathrm{C}$ & -2.86559400 & -1.21297400 & -2.73127600 \\
\hline $\mathrm{C}$ & 3.60053300 & -0.61479900 & 0.55515700 \\
\hline $\mathrm{C}$ & -3.65989300 & -1.30222800 & 0.01697000 \\
\hline $\mathrm{C}$ & -2.55598200 & 1.70187400 & -0.25984900 \\
\hline $\mathrm{C}$ & 1.03431000 & 4.23947300 & -0.81301400 \\
\hline $\mathrm{H}$ & 1.70622300 & 3.49355000 & -1.23370300 \\
\hline $\mathrm{C}$ & 2.71603500 & 1.22287100 & -2.84666000 \\
\hline $\mathrm{H}$ & 1.89429400 & 0.56591000 & -3.11032800 \\
\hline $\mathrm{C}$ & 1.54337500 & -3.45464100 & -0.33074900 \\
\hline $\mathrm{C}$ & 3.60242700 & -0.94841200 & 1.91523500 \\
\hline $\mathrm{H}$ & 2.86526400 & -0.52402800 & 2.59277700 \\
\hline $\mathrm{B}$ & 0.70265100 & -0.45056700 & -0.76480800 \\
\hline $\mathrm{C}$ & 0.15820600 & 3.89796300 & 0.22483900 \\
\hline $\mathrm{C}$ & 0.22126000 & 3.09890900 & 3.42920900 \\
\hline $\mathrm{H}$ & 1.12586300 & 3.60624500 & 3.09723500 \\
\hline $\mathrm{C}$ & -0.86490000 & -3.55829500 & 3.68811600 \\
\hline $\mathrm{H}$ & -1.27221200 & -4.48394200 & 4.08279100 \\
\hline $\mathrm{C}$ & 4.55399700 & -1.19410900 & -0.29338200 \\
\hline $\mathrm{H}$ & 4.55639700 & -0.94700000 & -1.35272400 \\
\hline $\mathrm{C}$ & -1.70015400 & 1.71512400 & 2.95701600 \\
\hline $\mathrm{H}$ & -2.27958100 & 1.11252400 & 2.26203800 \\
\hline $\mathrm{C}$ & -0.52330000 & 2.33067800 & 2.52132400 \\
\hline $\mathrm{C}$ & -4.03052200 & -1.41789500 & -5.26530800 \\
\hline $\mathrm{H}$ & -4.47826800 & -1.49868700 & -6.25131300 \\
\hline $\mathrm{C}$ & -3.17707600 & -0.06252200 & -3.46254200 \\
\hline
\end{tabular}




\begin{tabular}{|c|c|c|c|}
\hline $\mathrm{H}$ & -2.96970300 & 0.91599600 & -3.03669700 \\
\hline $\mathrm{C}$ & -0.71112400 & 6.15563600 & 0.19855200 \\
\hline $\mathrm{H}$ & -1.39698900 & 6.89943400 & 0.59281900 \\
\hline $\mathrm{C}$ & 3.12927200 & 1.32898800 & -1.51706200 \\
\hline $\mathrm{C}$ & -3.73323400 & -2.57007800 & -4.54033300 \\
\hline $\mathrm{H}$ & -3.95424800 & -3.54869700 & -4.95639100 \\
\hline $\mathrm{C}$ & 4.82099900 & 2.92972900 & -2.17457300 \\
\hline $\mathrm{H}$ & 5.64601800 & 3.58561300 & -1.91399400 \\
\hline $\mathrm{C}$ & 4.38576900 & 2.83918900 & -3.49737100 \\
\hline $\mathrm{H}$ & 4.86858500 & 3.43359900 & -4.26736700 \\
\hline $\mathrm{C}$ & -0.19885900 & 3.23631700 & 4.74705800 \\
\hline $\mathrm{H}$ & 0.38501400 & 3.83461900 & 5.44032200 \\
\hline $\mathrm{C}$ & -0.53043000 & -3.47097500 & 2.34099400 \\
\hline $\mathrm{H}$ & -0.67645600 & -4.33314300 & 1.69259900 \\
\hline $\mathrm{C}$ & 4.55542700 & -1.82824400 & 2.42008100 \\
\hline $\mathrm{H}$ & 4.55636100 & -2.06863100 & 3.47905000 \\
\hline $\mathrm{C}$ & 5.50136300 & -2.07487700 & 0.21587600 \\
\hline $\mathrm{H}$ & 6.23946100 & -2.51263900 & -0.44950600 \\
\hline $\mathrm{C}$ & -0.68291900 & -2.45941700 & 4.52752000 \\
\hline $\mathrm{H}$ & -0.95372700 & -2.52917900 & 5.57683600 \\
\hline $\mathrm{C}$ & -5.87501000 & -1.62735800 & 1.70187600 \\
\hline $\mathrm{H}$ & -6.73461800 & -1.75165700 & 2.35387200 \\
\hline $\mathrm{C}$ & 3.34167700 & 1.98394100 & -3.83196300 \\
\hline $\mathrm{H}$ & 3.00300100 & 1.90969700 & -4.86066100 \\
\hline $\mathrm{C}$ & 2.24985400 & -4.13524600 & 0.66708900 \\
\hline $\mathrm{H}$ & 2.05301900 & -3.92556000 & 1.71510400 \\
\hline $\mathrm{C}$ & -4.95603600 & -1.29793400 & -0.50773000 \\
\hline $\mathrm{H}$ & -5.11391000 & -1.16890400 & -1.57502700 \\
\hline $\mathrm{C}$ & -3.75841200 & -0.16507100 & -4.72326000 \\
\hline $\mathrm{H}$ & -3.99517800 & 0.73508300 & -5.28216700 \\
\hline $\mathrm{C}$ & -1.37426000 & 2.61758600 & 5.17237100 \\
\hline $\mathrm{H}$ & -1.70583600 & 2.73201000 & 6.20040000 \\
\hline $\mathrm{C}$ & 5.50698500 & -2.38955100 & 1.57293400 \\
\hline $\mathrm{H}$ & 6.25261200 & -3.07198300 & 1.96988200 \\
\hline $\mathrm{C}$ & 1.04760900 & 5.53121900 & -1.32627100 \\
\hline $\mathrm{H}$ & 1.73831100 & 5.78210300 & -2.12671600 \\
\hline
\end{tabular}




\begin{tabular}{|c|c|c|c|}
\hline $\mathrm{C}$ & 0.17561100 & -1.18749900 & 2.67346900 \\
\hline $\mathrm{H}$ & 0.53433200 & -0.24885600 & 2.26374700 \\
\hline $\mathrm{C}$ & 2.79309000 & -4.66405900 & -2.01676300 \\
\hline $\mathrm{H}$ & 3.00249000 & -4.86864600 & -3.06236200 \\
\hline C & 3.48413800 & -5.34690700 & -1.01676800 \\
\hline 11 & 4.23335700 & -6.08667100 & -1.28283500 \\
\hline $\mathrm{C}$ & 3.21469700 & -5.07752100 & 0.32116200 \\
\hline $\mathrm{H}$ & 3.75677500 & -5.60046200 & 1.10373400 \\
\hline $\mathrm{C}$ & -3.15935600 & -2.46964800 & -3.27729000 \\
\hline 11 & -2.95797900 & -3.37529600 & -2.70854600 \\
\hline$C$ & -3.48801900 & -1.46021600 & 1.39926600 \\
\hline $\mathrm{H}$ & -2.48906200 & -1.43208900 & 1.82887600 \\
\hline $\mathrm{C}$ & -4.58584200 & -1.63450400 & 2.23280300 \\
\hline $\mathrm{H}$ & -4.43067400 & -1.76882200 & 3.30065800 \\
\hline $\mathrm{C}$ & 4.19628900 & 2.17982500 & -1.18769500 \\
\hline $\mathrm{H}$ & 4.54037500 & 2.25160400 & -0.15719000 \\
\hline $\mathrm{C}$ & 1.83304700 & -3.71917100 & -1.67764800 \\
\hline $\mathrm{H}$ & 1.31143700 & -3.17788000 & -2.46512100 \\
\hline $\mathrm{C}$ & -6.05546600 & -1.45321000 & 0.33410300 \\
\hline $\mathrm{H}$ & -7.05683200 & -1.43946600 & -0.08618400 \\
\hline $\mathrm{C}$ & -2.12390400 & 1.86171800 & 4.27624400 \\
\hline $\mathrm{H}$ & -3.04315800 & 1.38104900 & 4.59983900 \\
\hline $\mathrm{C}$ & -0.15855900 & -1.27275800 & 4.02107300 \\
\hline $\mathrm{H}$ & -0.02701900 & -0.40529200 & 4.66263100 \\
\hline $\mathrm{H}$ & 0.90786200 & -0.83043400 & -1.89763000 \\
\hline $\mathrm{H}$ & -1.20375200 & 0.02542200 & 0.80214900 \\
\hline $\mathrm{C}$ & -1.20100200 & -2.58448900 & -0.81487400 \\
\hline $\mathrm{H}$ & -0.91740900 & -2.96439500 & -1.80212400 \\
\hline $\mathrm{H}$ & -1.76210300 & -3.36790500 & -0.29725600 \\
\hline$C$ & 1.85284600 & 1.70540200 & 1.04385600 \\
\hline $\mathrm{H}$ & 2.50313500 & 2.58081800 & 0.94959200 \\
\hline $\mathrm{H}$ & 1.97716400 & 1.31988800 & 2.06031500 \\
\hline
\end{tabular}

\section{3b}

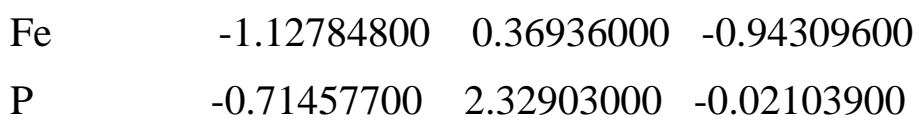




\begin{tabular}{|c|c|c|c|}
\hline $\mathrm{P}$ & -1.44668300 & -1.81569700 & -0.99315400 \\
\hline $\mathrm{P}$ & 0.82774100 & -1.70444500 & 0.86234200 \\
\hline $\mathrm{P}$ & 2.03383900 & 1.15419300 & -0.11534100 \\
\hline $\mathrm{O}$ & -0.48919600 & 1.09757700 & -3.71147100 \\
\hline $\mathrm{O}$ & -3.99194600 & 0.95604200 & -1.12160000 \\
\hline $\mathrm{C}$ & -0.71402900 & 0.79047900 & -2.60700500 \\
\hline $\mathrm{C}$ & -2.18291700 & 4.35369800 & -1.26126900 \\
\hline $\mathrm{H}$ & -2.99387900 & 4.03435500 & -0.61005500 \\
\hline $\mathrm{C}$ & 0.93258700 & -1.13593300 & 2.57965400 \\
\hline $\mathrm{C}$ & -1.37249700 & 5.77017300 & -3.04202000 \\
\hline $\mathrm{H}$ & -1.54925500 & 6.54934000 & -3.77799500 \\
\hline $\mathrm{C}$ & -0.69126800 & -2.90622400 & -2.26700700 \\
\hline $\mathrm{C}$ & 3.51988200 & 0.92373400 & 0.92136000 \\
\hline $\mathrm{C}$ & -3.19039000 & -2.34762100 & -1.06635300 \\
\hline $\mathrm{C}$ & -2.85500400 & 0.72842800 & -1.06269100 \\
\hline $\mathrm{C}$ & 0.10627200 & 4.17995500 & -1.99380300 \\
\hline $\mathrm{H}$ & 1.08692800 & 3.71872200 & -1.93621100 \\
\hline $\mathrm{C}$ & 2.48908700 & 1.29992300 & -2.89150200 \\
\hline $\mathrm{H}$ & 1.90558400 & 0.38900000 & -2.96769900 \\
\hline $\mathrm{C}$ & 2.17254000 & -2.90426200 & 0.60389200 \\
\hline $\mathrm{C}$ & 3.46782500 & 0.99204900 & 2.31918800 \\
\hline $\mathrm{H}$ & 2.55348600 & 1.28644900 & 2.82716900 \\
\hline B & 0.90338000 & -0.33828800 & -0.50402100 \\
\hline $\mathrm{C}$ & -0.91905400 & 3.76016200 & -1.14189100 \\
\hline $\mathrm{C}$ & -1.48020300 & 4.12758900 & 1.98648300 \\
\hline $\mathrm{H}$ & -0.80780300 & 4.82920500 & 1.49421000 \\
\hline $\mathrm{C}$ & 1.78488300 & -1.25157400 & 4.83905400 \\
\hline $\mathrm{H}$ & 2.38447300 & -1.75894700 & 5.58973600 \\
\hline $\mathrm{C}$ & 4.71151000 & 0.51309200 & 0.30448700 \\
\hline $\mathrm{H}$ & 4.76366400 & 0.44726400 & -0.77972700 \\
\hline $\mathrm{C}$ & -2.55445100 & 1.96252500 & 2.07717700 \\
\hline $\mathrm{H}$ & -2.70269200 & 0.94806000 & 1.71342600 \\
\hline $\mathrm{C}$ & -1.67650800 & 2.84501800 & 1.44513700 \\
\hline $\mathrm{C}$ & 0.51913400 & -4.65796000 & -4.08935900 \\
\hline $\mathrm{H}$ & 0.99198400 & -5.33691500 & -4.79374300 \\
\hline $\mathrm{C}$ & 0.18153700 & -2.42342700 & $\begin{array}{c}-3.24245400 \\
\text { S39 }\end{array}$ \\
\hline
\end{tabular}




\begin{tabular}{|c|c|c|c|}
\hline $\mathrm{H}$ & 0.38902800 & -1.35875500 & -3.28844400 \\
\hline $\mathrm{C}$ & -2.40410000 & 5.35225600 & -2.20429800 \\
\hline $\mathrm{H}$ & -3.38851600 & 5.80482100 & -2.28407700 \\
\hline $\mathrm{C}$ & 2.72867000 & 1.86283100 & -1.63809100 \\
\hline $\mathrm{C}$ & -0.36888700 & -5.15090100 & -3.13339100 \\
\hline $\mathrm{H}$ & -0.59530800 & -6.21300800 & -3.09388900 \\
\hline $\mathrm{C}$ & 4.00227600 & 3.63393100 & -2.69067100 \\
\hline $\mathrm{H}$ & 4.59852700 & 4.53820600 & -2.60990500 \\
\hline $\mathrm{C}$ & 3.73035100 & 3.08166100 & -3.94321100 \\
\hline $\mathrm{H}$ & 4.10865300 & 3.56186900 & -4.84139300 \\
\hline $\mathrm{C}$ & -2.15370800 & 4.50911000 & 3.13939200 \\
\hline $\mathrm{H}$ & -2.00064700 & 5.50407000 & 3.54924200 \\
\hline $\mathrm{C}$ & 1.69493200 & -1.78537200 & 3.55753900 \\
\hline $\mathrm{H}$ & 2.22680600 & -2.70215100 & 3.32005300 \\
\hline $\mathrm{C}$ & 4.58163800 & 0.65134600 & 3.08161100 \\
\hline $\mathrm{H}$ & 4.51816400 & 0.70067200 & 4.16495200 \\
\hline $\mathrm{C}$ & 5.82371400 & 0.18225100 & 1.07024400 \\
\hline $\mathrm{H}$ & 6.73992900 & -0.13242800 & 0.57798400 \\
\hline $\mathrm{C}$ & 1.10455800 & -0.07737500 & 5.16170700 \\
\hline $\mathrm{H}$ & 1.17732000 & 0.33294900 & 6.16531300 \\
\hline $\mathrm{C}$ & -5.93205100 & -2.83532500 & -1.28445800 \\
\hline $\mathrm{H}$ & -6.99931900 & -3.02355100 & -1.36837200 \\
\hline $\mathrm{C}$ & 2.98101500 & 1.91459300 & -4.04081100 \\
\hline $\mathrm{H}$ & 2.76646600 & 1.47999100 & -5.01258100 \\
\hline $\mathrm{C}$ & 3.46952100 & -2.60866800 & 1.04912900 \\
\hline $\mathrm{H}$ & 3.64654600 & -1.73162700 & 1.66761100 \\
\hline $\mathrm{C}$ & -3.77086000 & -2.57863700 & -2.32166900 \\
\hline $\mathrm{H}$ & -3.15489000 & -2.56539600 & -3.21866800 \\
\hline $\mathrm{C}$ & 0.78509000 & -3.29406600 & -4.14810600 \\
\hline $\mathrm{H}$ & 1.46439400 & -2.90287400 & -4.90047900 \\
\hline $\mathrm{C}$ & -3.02113300 & 3.61463400 & 3.77175700 \\
\hline $\mathrm{H}$ & -3.53911100 & 3.91438700 & 4.67918900 \\
\hline $\mathrm{C}$ & 5.76014200 & 0.24495500 & 2.46081300 \\
\hline $\mathrm{H}$ & 6.62743000 & -0.02043400 & 3.05848300 \\
\hline $\mathrm{C}$ & -0.11628200 & 5.18099300 & -2.93385800 \\
\hline $\mathrm{H}$ & 0.69701400 & 5.49340500 & $\begin{array}{c}-3.58421400 \\
\text { S40 }\end{array}$ \\
\hline
\end{tabular}




\begin{tabular}{|c|c|c|c|}
\hline $\mathrm{C}$ & 0.20809700 & 0.01087300 & 2.92914300 \\
\hline $\mathrm{H}$ & -0.44558600 & 0.47534800 & 2.19922600 \\
\hline $\mathrm{C}$ & 3.04799400 & -4.85133800 & -0.54327200 \\
\hline $\mathrm{H}$ & 2.87332300 & -5.71930600 & -1.17370200 \\
\hline $\mathrm{C}$ & 4.32905100 & -4.55763800 & -0.08678500 \\
\hline $\mathrm{H}$ & 5.16485400 & -5.19821400 & -0.35397100 \\
\hline $\mathrm{C}$ & 4.53599300 & -3.43427900 & 0.71045500 \\
\hline $\mathrm{H}$ & 5.53272000 & -3.19196300 & 1.07055800 \\
\hline $\mathrm{C}$ & -0.97264500 & -4.28108100 & -2.23287900 \\
\hline $\mathrm{H}$ & -1.67653100 & -4.66793600 & -1.49750000 \\
\hline $\mathrm{C}$ & -3.99386100 & -2.34687100 & 0.07818300 \\
\hline $\mathrm{H}$ & -3.57868900 & -2.12078300 & 1.06385000 \\
\hline $\mathrm{C}$ & -5.35847600 & -2.59912700 & -0.03891300 \\
\hline $\mathrm{H}$ & -5.97040100 & -2.59796600 & 0.85887300 \\
\hline $\mathrm{C}$ & 3.50422400 & 3.02898200 & -1.54438500 \\
\hline $\mathrm{H}$ & 3.71511100 & 3.46292700 & -0.56812100 \\
\hline $\mathrm{C}$ & 1.97674300 & -4.03200900 & -0.20022800 \\
\hline $\mathrm{H}$ & 0.98918200 & -4.26672900 & -0.58696100 \\
\hline $\mathrm{C}$ & -5.13509700 & -2.82266800 & -2.42768900 \\
\hline $\mathrm{H}$ & -5.57619500 & -2.99956900 & -3.40497400 \\
\hline $\mathrm{C}$ & -3.21396400 & 2.34302200 & 3.24532300 \\
\hline $\mathrm{H}$ & -3.84945600 & 1.60948800 & 3.73442200 \\
\hline $\mathrm{C}$ & 0.30496100 & 0.54586500 & 4.20976600 \\
\hline $\mathrm{H}$ & -0.28066900 & 1.42669700 & 4.46174300 \\
\hline $\mathrm{H}$ & 1.46422100 & -0.91570400 & -1.40301100 \\
\hline $\mathrm{H}$ & -1.38508100 & -0.02573200 & 0.51403400 \\
\hline $\mathrm{C}$ & -0.77084600 & -2.53299900 & 0.57116900 \\
\hline $\mathrm{H}$ & -0.70359900 & -3.62511200 & 0.58255600 \\
\hline $\mathrm{H}$ & -1.43359100 & -2.21474800 & 1.40857600 \\
\hline $\mathrm{C}$ & 1.03761900 & 2.48968400 & 0.62869200 \\
\hline $\mathrm{H}$ & 1.46871400 & 3.48194200 & 0.45748600 \\
\hline $\mathrm{H}$ & 0.98525300 & 2.32668400 & 1.71078000 \\
\hline $\mathrm{Br}$ & -2.74053600 & -1.30525300 & 3.28341500 \\
\hline
\end{tabular}

3biso1

Fe $\quad \begin{array}{llll}0.67962200 & -1.26114000 & -0.95669000\end{array}$ 


\begin{tabular}{|c|c|c|c|}
\hline $\mathrm{P}$ & -0.85325500 & -2.07333500 & 0.43061400 \\
\hline $\mathrm{P}$ & 2.56778400 & -0.14180000 & -0.64450300 \\
\hline $\mathrm{P}$ & 0.60313800 & 2.31756200 & -0.33250800 \\
\hline $\mathrm{P}$ & -2.22317500 & 0.60882100 & -0.02851800 \\
\hline $\mathrm{O}$ & -0.04282300 & -1.54203000 & -3.78207600 \\
\hline $\mathrm{O}$ & 2.08343100 & -3.78590600 & -0.74680900 \\
\hline $\mathrm{C}$ & 0.24992400 & -1.39287500 & -2.65864000 \\
\hline $\mathrm{C}$ & -1.77406100 & -3.91153400 & -1.43378500 \\
\hline $\mathrm{H}$ & -0.77127800 & -3.81538700 & -1.84291500 \\
\hline $\mathrm{C}$ & 0.44220500 & 2.51203500 & 1.46032700 \\
\hline $\mathrm{C}$ & -3.99720900 & -4.83793700 & -1.56029100 \\
\hline $\mathrm{H}$ & -4.72476700 & -5.47569300 & -2.05404800 \\
\hline $\mathrm{C}$ & 3.90421000 & -0.32531500 & -1.88885800 \\
\hline $\mathrm{C}$ & -2.85921000 & 2.04688600 & 0.86853700 \\
\hline $\mathrm{C}$ & 3.54681500 & -0.35240700 & 0.89557300 \\
\hline $\mathrm{C}$ & 1.52371900 & -2.76210000 & -0.83480500 \\
\hline $\mathrm{C}$ & -3.42975200 & -3.29446000 & 0.20448600 \\
\hline $\mathrm{H}$ & -3.73633700 & -2.74433600 & 1.09171900 \\
\hline $\mathrm{C}$ & -3.21624900 & -0.71875200 & -2.25822800 \\
\hline $\mathrm{H}$ & -2.17735400 & -0.85462000 & -2.54632100 \\
\hline $\mathrm{C}$ & 0.41383700 & 3.92740400 & -1.11975300 \\
\hline $\mathrm{C}$ & -3.07758600 & 2.02876900 & 2.24889800 \\
\hline $\mathrm{H}$ & -2.88352500 & 1.12770700 & 2.82543000 \\
\hline B & -0.54834400 & 0.89236800 & -0.95928500 \\
\hline $\mathrm{C}$ & -2.12503100 & -3.17780700 & -0.29361900 \\
\hline $\mathrm{C}$ & -1.06404500 & -3.87403300 & 2.65303300 \\
\hline $\mathrm{H}$ & -1.99347200 & -4.24263400 & 2.22490700 \\
\hline $\mathrm{C}$ & -0.21439900 & 3.75908200 & 3.42252700 \\
\hline $\mathrm{H}$ & -0.57286800 & 4.68206800 & 3.86886700 \\
\hline $\mathrm{C}$ & -3.10175900 & 3.22204300 & 0.14341800 \\
\hline $\mathrm{H}$ & -2.94914600 & 3.23827900 & -0.93404700 \\
\hline $\mathrm{C}$ & 0.88875900 & -2.45863100 & 2.53420300 \\
\hline $\mathrm{H}$ & 1.48832100 & -1.73402200 & 1.98230200 \\
\hline $\mathrm{C}$ & -0.32293900 & -2.89442000 & 1.98431200 \\
\hline$C$ & 5.91034900 & -0.57851500 & -3.81674900 \\
\hline $\mathrm{H}$ & 6.69171500 & -0.68016000 & $\begin{array}{c}-4.56415600 \\
\text { S42 }\end{array}$ \\
\hline
\end{tabular}




\begin{tabular}{|c|c|c|c|}
\hline $\mathrm{C}$ & 5.16552400 & 0.24950300 & -1.67763500 \\
\hline $\mathrm{H}$ & 5.37190900 & 0.77864600 & -0.74848300 \\
\hline $\mathrm{C}$ & -2.69997200 & -4.74178800 & -2.05683600 \\
\hline $\mathrm{H}$ & -2.40866500 & -5.30611300 & -2.93766800 \\
\hline $\mathrm{C}$ & -3.53855000 & 0.00729200 & -1.10589400 \\
\hline $\mathrm{C}$ & 4.66464500 & -1.16088800 & -4.02782500 \\
\hline $\mathrm{H}$ & 4.46975100 & -1.72020700 & -4.93801600 \\
\hline $\mathrm{C}$ & -5.88469700 & -0.40347200 & -1.49368700 \\
\hline $\mathrm{H}$ & -6.92304900 & -0.27505400 & -1.20312700 \\
\hline $\mathrm{C}$ & -5.56154100 & -1.13537700 & -2.63516800 \\
\hline $\mathrm{H}$ & -6.35176700 & -1.58278900 & -3.23108000 \\
\hline $\mathrm{C}$ & -0.60245300 & -4.39299800 & 3.86090600 \\
\hline $\mathrm{H}$ & -1.17690900 & -5.16160000 & 4.36996700 \\
\hline $\mathrm{C}$ & -0.01936100 & 3.69620800 & 2.04606700 \\
\hline $\mathrm{H}$ & -0.24420800 & 4.56205700 & 1.42897000 \\
\hline $\mathrm{C}$ & -3.53695700 & 3.17389600 & 2.89445900 \\
\hline $\mathrm{H}$ & -3.70601200 & 3.15339200 & 3.96714400 \\
\hline $\mathrm{C}$ & -3.55247700 & 4.36413400 & 0.79433300 \\
\hline $\mathrm{H}$ & -3.73937100 & 5.27051000 & 0.22596400 \\
\hline $\mathrm{C}$ & 0.03860000 & 2.64685700 & 4.22217800 \\
\hline $\mathrm{H}$ & -0.11133300 & 2.70498700 & 5.29627500 \\
\hline $\mathrm{C}$ & 4.94333000 & -0.94631800 & 3.25989800 \\
\hline $\mathrm{H}$ & 5.48396100 & -1.17532200 & 4.17359300 \\
\hline $\mathrm{C}$ & -4.23127200 & -1.29207500 & -3.01621400 \\
\hline $\mathrm{H}$ & -3.97564000 & -1.86724000 & -3.90095800 \\
\hline $\mathrm{C}$ & -0.52016400 & 4.08641000 & -2.15009300 \\
\hline $\mathrm{H}$ & -1.13099400 & 3.24160000 & -2.46076400 \\
\hline $\mathrm{C}$ & 4.10452300 & -1.62554600 & 1.10142000 \\
\hline $\mathrm{H}$ & 3.99012600 & -2.39516300 & 0.34148600 \\
\hline $\mathrm{C}$ & 6.16029600 & 0.12762400 & -2.64070600 \\
\hline $\mathrm{H}$ & 7.13504600 & 0.57559400 & -2.47079400 \\
\hline $\mathrm{C}$ & 0.59216900 & -3.93596300 & 4.41272600 \\
\hline $\mathrm{H}$ & 0.94627600 & -4.34691300 & 5.35370300 \\
\hline $\mathrm{C}$ & -3.77159600 & 4.33960800 & 2.17116100 \\
\hline $\mathrm{H}$ & -4.12916600 & 5.23055100 & 2.67898500 \\
\hline $\mathrm{C}$ & -4.36033500 & -4.11290100 & $\begin{array}{c}-0.42816800 \\
\text { S43 }\end{array}$ \\
\hline
\end{tabular}




$\begin{array}{lrrr}\mathrm{H} & -5.37063400 & -4.18403600 & -0.03480100 \\ \mathrm{C} & 0.67866900 & 1.38886800 & 2.26820700 \\ \mathrm{H} & 1.01729300 & 0.45380700 & 1.82077400 \\ \mathrm{C} & 1.09240900 & 6.23218500 & -1.38823600 \\ \mathrm{H} & 1.72322800 & 7.06627500 & -1.09728800 \\ \mathrm{C} & 0.15112000 & 6.38751300 & -2.40526400 \\ \mathrm{H} & 0.04915000 & 7.34626000 & -2.90451900 \\ \mathrm{C} & -0.65099100 & 5.31628500 & -2.78813000 \\ \mathrm{H} & -1.37478000 & 5.43455000 & -3.58842100 \\ \mathrm{C} & 3.66286400 & -1.03264100 & -3.06854100 \\ \mathrm{H} & 2.68755400 & -1.48353300 & -3.22865200 \\ \mathrm{C} & 3.71132000 & 0.61582400 & 1.89155200 \\ \mathrm{H} & 3.29673400 & 1.61321300 & 1.76838800 \\ \mathrm{C} & 4.39680700 & 0.31802200 & 3.06893600 \\ \mathrm{H} & 4.51077000 & 1.08407200 & 3.83102500 \\ \mathrm{C} & -4.87703700 & 0.16603300 & -0.72400500 \\ \mathrm{H} & -5.12761200 & 0.73941300 & 0.16633700 \\ \mathrm{C} & 1.22812600 & 5.00702900 & -0.74667300 \\ \mathrm{H} & 1.96274700 & 4.88927700 & 0.04759800 \\ \mathrm{C} & 4.79962100 & -1.91654900 & 2.26742600 \\ \mathrm{H} & 5.22779500 & -2.90530000 & 2.40364400 \\ \mathrm{H} & 1.33982100 & -2.96685700 & 3.74764100 \\ \mathrm{H} & 2.28426300 & -2.61870200 & 4.15862600 \\ \mathrm{H} & 0.48065200 & 1.45885200 & 3.64278400 \\ \mathrm{H} & 0.68363000 & 0.58238900 & 4.25338400 \\ \mathrm{H} & -0.66639400 & 0.94387800 & -2.14392900 \\ \mathrm{H} & 0.13972100 & 0.10061400 & -0.24935900 \\ \mathrm{H} & 2.28380300 & 1.71667400 & -0.74732400 \\ \mathrm{H} & 2.40451200 & 1.95794100 & -1.80973200 \\ \mathrm{H} & -1.879291000 & -0.69993800 & 1.18708100 \\ \mathrm{H} & -2.8069900 & -1.06328800 & 1.63933400\end{array}$

$3 b_{\text {iso2 }}$

Fe $\quad \begin{array}{llll}0.16296000 & -0.92691100 & -1.42267500\end{array}$ 


\begin{tabular}{|c|c|c|c|}
\hline $\mathrm{P}$ & -1.18035200 & -1.60305800 & 0.25493100 \\
\hline$P$ & -2.09059900 & 1.11874100 & -0.14914100 \\
\hline $\mathrm{P}$ & 0.98259100 & 1.79723600 & 0.32733100 \\
\hline$P$ & 2.07857300 & -0.86521000 & -0.23520600 \\
\hline . & -3.55828600 & -2.97885800 & 0.75546400 \\
\hline $\mathrm{H}$ & -3.31613700 & -2.80861600 & 1.80402200 \\
\hline $\mathrm{C}$ & -3.37022500 & 0.63803500 & -1.33526400 \\
\hline $\mathrm{C}$ & 3.68207200 & -0.29479000 & -0.92632900 \\
\hline 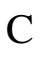 & -2.76143500 & 2.61204700 & 0.63814800 \\
\hline$C$ & -3.02309600 & 2.69527000 & 2.00830400 \\
\hline $\mathrm{H}$ & -2.82488500 & 1.84794200 & 2.66014600 \\
\hline $\mathrm{C}$ & -0.57573700 & -2.21612500 & 2.9866500 \\
\hline $\mathrm{H}$ & -0.91578100 & -1.22039200 & 3.2647850 \\
\hline $\mathrm{C}$ & -2.98173800 & -2.80693100 & -1.5813370 \\
\hline $\mathrm{H}$ & -2.30883400 & -2.46943100 & -2.3639350 \\
\hline 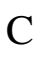 & 0.11382400 & 4.19723300 & 1.40104900 \\
\hline $\mathrm{H}$ & 0.19948500 & 4.56069600 & 0.37814300 \\
\hline $\mathrm{C}$ & 0.45800200 & 2.87119100 & 1.70016600 \\
\hline$C$ & 2.84807500 & -2.38976100 & 2.05098800 \\
\hline $\mathrm{H}$ & 2.59446800 & -1.53468600 & 2.67350600 \\
\hline $\mathrm{C}$ & 2.98466500 & -3.46981000 & -0.10227000 \\
\hline $\mathrm{H}$ & 2.84580000 & -3.44799700 & -1.18100600 \\
\hline $\mathrm{C}$ & 4.82294100 & -0.31215000 & -0.11047700 \\
\hline $\mathrm{H}$ & 4.75831300 & -0.70112600 & 0.90456600 \\
\hline$C$ & -0.59347500 & -2.63194900 & 1.65152800 \\
\hline $\mathrm{C}$ & -2.67596600 & -2.54439500 & -0.24458100 \\
\hline $\mathrm{C}$ & -3.53362400 & 4.88870800 & 0.36245200 \\
\hline $\mathrm{H}$ & -3.73789300 & 5.73970200 & -0.28028700 \\
\hline $\mathrm{C}$ & 3.37222900 & -3.53189800 & 2.65154000 \\
\hline $\mathrm{H}$ & 3.51674500 & -3.55329500 & 3.72832700 \\
\hline $\mathrm{C}$ & -3.14585700 & 0.74718500 & -2.71135300 \\
\hline 111 & -2.17679000 & 1.07716600 & -3.07855000 \\
\hline $\mathrm{C}$ & -4.61382800 & 0.20368300 & -0.86176700 \\
\hline $\mathrm{H}$ & -4.79829000 & 0.12505600 & 0.20843600 \\
\hline 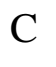 & -0.42800700 & 4.58981700 & 3.71713800 \\
\hline $\mathrm{H}$ & -0.76746500 & 5.25988900 & 4.50165500 \\
\hline
\end{tabular}




\begin{tabular}{|c|c|c|c|}
\hline $\mathrm{C}$ & -3.01727700 & 3.71997600 & -0.18253300 \\
\hline $\mathrm{H}$ & -2.82242500 & 3.66004800 & -1.25214800 \\
\hline $\mathrm{C}$ & -3.79935000 & 4.96325100 & 1.72891100 \\
\hline $\mathrm{H}$ & -4.20975200 & 5.87495800 & 2.15292200 \\
\hline $\mathrm{C}$ & -0.09372200 & 3.27467100 & 4.02271800 \\
\hline $\mathrm{H}$ & -0.16263400 & 2.91712100 & 5.04596300 \\
\hline $\mathrm{C}$ & 3.49116400 & 2.98012200 & 0.16839600 \\
\hline $\mathrm{H}$ & 3.62827500 & 2.62677300 & 1.18852700 \\
\hline $\mathrm{C}$ & 5.01532000 & 0.63392700 & -2.71863200 \\
\hline $\mathrm{H}$ & 5.08487900 & 1.00095900 & -3.73866900 \\
\hline $\mathrm{C}$ & 3.50629900 & -4.60852000 & 0.50016200 \\
\hline $\mathrm{H}$ & 3.76391400 & -5.46949900 & -0.10928300 \\
\hline $\mathrm{C}$ & 2.29691900 & 2.71798800 & -0.51731200 \\
\hline $\mathrm{C}$ & 2.65495900 & -2.34599200 & 0.66761300 \\
\hline $\mathrm{C}$ & -4.73324500 & -3.64002600 & 0.42160600 \\
\hline $\mathrm{H}$ & -5.40914400 & -3.97025200 & 1.20513200 \\
\hline $\mathrm{C}$ & 6.04221600 & 0.14623700 & -0.59349600 \\
\hline $\mathrm{H}$ & 6.91940200 & 0.12444500 & 0.04665700 \\
\hline $\mathrm{C}$ & -4.15791000 & 0.40997600 & -3.60480500 \\
\hline $\mathrm{H}$ & -3.98160500 & 0.49252700 & -4.67291400 \\
\hline $\mathrm{C}$ & 0.24688300 & -4.79656500 & 2.34273100 \\
\hline $\mathrm{H}$ & 0.57245100 & -5.80027400 & 2.08568700 \\
\hline $\mathrm{C}$ & 3.70418800 & -4.64053100 & 1.87898900 \\
\hline $\mathrm{H}$ & 4.11693800 & -5.52831100 & 2.34956600 \\
\hline $\mathrm{C}$ & -0.16643500 & -3.93131600 & 1.33974400 \\
\hline $\mathrm{H}$ & -0.16302300 & -4.26685800 & 0.30391900 \\
\hline $\mathrm{C}$ & -0.32777800 & 5.04858700 & 2.40416600 \\
\hline $\mathrm{H}$ & -0.59459900 & 6.07296900 & 2.16201300 \\
\hline $\mathrm{C}$ & -4.15543300 & -3.48034800 & -1.91310100 \\
\hline $\mathrm{H}$ & -4.38226400 & -3.67725300 & -2.95689800 \\
\hline $\mathrm{C}$ & 0.34667600 & 2.41424100 & 3.01845200 \\
\hline $\mathrm{H}$ & 0.62550800 & 1.39436100 & 3.27619300 \\
\hline $\mathrm{C}$ & 0.25055100 & -4.37680100 & 3.67274900 \\
\hline $\mathrm{H}$ & 0.57258600 & -5.05624000 & 4.45667100 \\
\hline B & -0.34814100 & 1.18764000 & -0.92508700 \\
\hline $\mathrm{C}$ & 2.13529700 & 3.18820600 & -1.82397000 \\
\hline
\end{tabular}




\begin{tabular}{|c|c|c|c|}
\hline $\mathrm{H}$ & 1.22371500 & 2.97442400 & -2.37279300 \\
\hline $\mathrm{C}$ & -3.54166100 & 3.86882600 & 2.54886700 \\
\hline $\mathrm{H}$ & -3.74481100 & 3.92454700 & 3.61460000 \\
\hline $\mathrm{C}$ & -5.03406000 & -3.89112700 & -0.91657800 \\
\hline $\mathrm{H}$ & -5.94905300 & -4.41482800 & -1.17826900 \\
\hline $\mathrm{C}$ & 3.79153300 & 0.17529300 & -2.23566400 \\
\hline $\mathrm{H}$ & 2.92045600 & 0.19403900 & -2.88112900 \\
\hline $\mathrm{C}$ & 4.51000100 & 3.69184700 & -0.45017700 \\
\hline $\mathrm{H}$ & 5.43750100 & 3.87788700 & 0.08315800 \\
\hline $\mathrm{C}$ & -5.38818800 & -0.03799500 & -3.13027700 \\
\hline $\mathrm{H}$ & -6.17325500 & -0.30707600 & -3.83099500 \\
\hline $\mathrm{C}$ & 6.13975200 & 0.62177900 & -1.90020200 \\
\hline $\mathrm{H}$ & 7.09369600 & 0.97574600 & -2.28026500 \\
\hline $\mathrm{C}$ & -5.61712000 & -0.13987200 & -1.75913300 \\
\hline $\mathrm{H}$ & -6.57465300 & -0.49257400 & -1.38816200 \\
\hline $\mathrm{C}$ & 4.34159300 & 4.16254700 & -1.75147600 \\
\hline $\mathrm{H}$ & 5.13916400 & 4.72046000 & -2.23303200 \\
\hline $\mathrm{C}$ & 3.15594600 & 3.91300900 & -2.43413300 \\
\hline $\mathrm{H}$ & 3.02292900 & 4.27388600 & -3.44955200 \\
\hline $\mathrm{C}$ & -0.15405800 & -3.08624700 & 3.99228000 \\
\hline $\mathrm{H}$ & -0.15671800 & -2.75491000 & 5.02677600 \\
\hline $\mathrm{H}$ & -0.40859900 & 1.99984200 & -1.80965700 \\
\hline $\mathrm{C}$ & 0.53753800 & -0.06349000 & -2.88813300 \\
\hline $\mathrm{C}$ & 0.35767500 & -2.56239300 & -2.04462500 \\
\hline $\mathrm{O}$ & 0.66997900 & 0.50357400 & -3.89814300 \\
\hline $\mathrm{O}$ & 0.44250600 & -3.63282100 & -2.49364000 \\
\hline $\mathrm{H}$ & -1.19828100 & -0.84501500 & -2.04528100 \\
\hline $\mathrm{C}$ & -1.97334400 & -0.16603700 & 1.12192200 \\
\hline $\mathrm{H}$ & -1.29421000 & 0.23896100 & 1.88492200 \\
\hline $\mathrm{H}$ & -2.91852900 & -0.43317000 & 1.60517700 \\
\hline $\mathrm{C}$ & 1.77938900 & 0.36132400 & 1.11525200 \\
\hline $\mathrm{H}$ & 2.66481500 & 0.63832200 & 1.69957400 \\
\hline $\mathrm{H}$ & 1.05014700 & -0.10740600 & 1.79011900 \\
\hline
\end{tabular}

$3 b_{\text {iso3 }}$

Fe $\quad \begin{array}{llll}0.52207200 & 0.54499500 & -0.57291400\end{array}$ 


\begin{tabular}{|c|c|c|c|}
\hline $\mathrm{P}$ & -0.87082200 & -0.96656300 & -1.50103900 \\
\hline $\mathrm{P}$ & 1.86714200 & 1.80078500 & 0.74429800 \\
\hline $\mathrm{P}$ & 1.53614800 & -0.87942800 & 0.96628000 \\
\hline $\mathrm{P}$ & -2.66117400 & 0.15805500 & 0.65616400 \\
\hline $\mathrm{O}$ & -0.89357400 & 2.93348900 & -1.43591100 \\
\hline $\mathrm{O}$ & 2.14827300 & 0.38266800 & -3.00525300 \\
\hline $\mathrm{C}$ & -0.37676800 & 1.94814000 & -1.09000400 \\
\hline C & -1.14347600 & -1.24115000 & -4.28754200 \\
\hline $\mathrm{H}$ & -0.48934200 & -2.10403200 & -4.19828300 \\
\hline $\mathrm{C}$ & 3.12042500 & -1.72479400 & 0.57691500 \\
\hline $\mathrm{C}$ & -2.44505100 & 0.24090300 & -5.67911500 \\
\hline $\mathrm{H}$ & -2.80335100 & 0.53582900 & -6.66093000 \\
\hline $\mathrm{C}$ & 1.32786500 & 3.34817900 & 1.53126200 \\
\hline $\mathrm{C}$ & -3.58489400 & -0.54308800 & 2.05810500 \\
\hline $\mathrm{C}$ & 3.52014100 & 2.21553300 & 0.09708300 \\
\hline $\mathrm{C}$ & 1.56181400 & 0.41667100 & -1.99990900 \\
\hline $\mathrm{C}$ & -2.35746500 & 0.59512700 & -3.29422700 \\
\hline $\mathrm{H}$ & -2.64666500 & 1.18598500 & -2.43058500 \\
\hline $\mathrm{C}$ & -3.64825500 & 2.62497400 & -0.18151400 \\
\hline $\mathrm{H}$ & -2.80549400 & 3.05147900 & 0.35465600 \\
\hline $\mathrm{C}$ & 0.71035200 & -2.08150000 & 2.07092100 \\
\hline $\mathrm{C}$ & -2.99761400 & -1.59669900 & 2.77020800 \\
\hline $\mathrm{H}$ & -2.02686300 & -1.98853100 & 2.47185100 \\
\hline B & -0.96288300 & 0.92572900 & 1.11944200 \\
\hline $\mathrm{C}$ & -1.52284600 & -0.52079600 & -3.14782900 \\
\hline $\mathrm{C}$ & -1.06600500 & -3.76716500 & -1.77502100 \\
\hline $\mathrm{H}$ & -2.14122800 & -3.63776100 & -1.67675700 \\
\hline $\mathrm{C}$ & 4.77206300 & -3.44970100 & 0.99167700 \\
\hline $\mathrm{H}$ & 5.07560800 & -4.34235100 & 1.53081100 \\
\hline $\mathrm{C}$ & -4.82068100 & -0.03615300 & 2.47395200 \\
\hline $\mathrm{H}$ & -5.28412100 & 0.78619300 & 1.93534900 \\
\hline $\mathrm{C}$ & 1.16062300 & -2.84959600 & -1.91740700 \\
\hline $\mathrm{H}$ & 1.83418800 & -1.99964300 & -1.90847500 \\
\hline $\mathrm{C}$ & -0.21506400 & -2.65286600 & -1.75684400 \\
\hline$C$ & 0.46833700 & 5.76730000 & 2.63082200 \\
\hline $\mathrm{H}$ & 0.13202600 & 6.70699000 & $\begin{array}{c}3.05847400 \\
\text { S48 }\end{array}$ \\
\hline
\end{tabular}




\begin{tabular}{|c|c|c|c|}
\hline $\mathrm{C}$ & 1.07764100 & 4.45110100 & 0.70488800 \\
\hline $\mathrm{H}$ & 1.21734200 & 4.37262600 & -0.37090200 \\
\hline $\mathrm{C}$ & -1.60176200 & -0.85723200 & -5.54467500 \\
\hline $\mathrm{H}$ & -1.29922800 & -1.42392700 & -6.42006400 \\
\hline $\mathrm{C}$ & -3.82770300 & 1.23845000 & -0.20823800 \\
\hline $\mathrm{C}$ & 0.72560900 & 4.67806200 & 3.45762900 \\
\hline $\mathrm{H}$ & 0.59500400 & 4.76516100 & 4.53206200 \\
\hline $\mathrm{C}$ & -5.80748000 & 1.52920400 & -1.57017900 \\
\hline $\mathrm{H}$ & -6.64992100 & 1.10232000 & -2.10592200 \\
\hline $\mathrm{C}$ & -5.60667000 & 2.90953500 & -1.56199600 \\
\hline $\mathrm{H}$ & -6.29261400 & 3.55900900 & -2.09798100 \\
\hline $\mathrm{C}$ & -0.54339900 & -5.04914600 & -1.91347400 \\
\hline $\mathrm{H}$ & -1.21173700 & -5.90515900 & -1.91835000 \\
\hline $\mathrm{C}$ & 3.53307700 & -2.87510800 & 1.26197300 \\
\hline $\mathrm{H}$ & 2.89216500 & -3.32749500 & 2.01279600 \\
\hline $\mathrm{C}$ & -3.64594000 & -2.14758900 & 3.86749100 \\
\hline $\mathrm{H}$ & -3.18211900 & -2.96948000 & 4.40603900 \\
\hline $\mathrm{C}$ & -5.46239000 & -0.58905800 & 3.57996700 \\
\hline $\mathrm{H}$ & -6.42216400 & -0.19192300 & 3.89641200 \\
\hline $\mathrm{C}$ & 5.61927900 & -2.88219200 & 0.04474400 \\
\hline $\mathrm{H}$ & 6.58676500 & -3.33096800 & -0.16023000 \\
\hline $\mathrm{C}$ & 6.02413000 & 2.85080100 & -0.96810500 \\
\hline $\mathrm{H}$ & 6.99912900 & 3.08598300 & -1.38448300 \\
\hline $\mathrm{C}$ & -4.53461600 & 3.45536400 & -0.86290900 \\
\hline $\mathrm{H}$ & -4.38185400 & 4.53007500 & -0.84897200 \\
\hline $\mathrm{C}$ & 0.27404200 & -3.30556800 & 1.54085700 \\
\hline $\mathrm{H}$ & 0.44594300 & -3.53891200 & 0.49452900 \\
\hline $\mathrm{C}$ & 4.66924100 & 2.12070000 & 0.89003100 \\
\hline $\mathrm{H}$ & 4.60018600 & 1.80305500 & 1.92772000 \\
\hline $\mathrm{C}$ & 0.64753000 & 5.65277000 & 1.25374000 \\
\hline $\mathrm{H}$ & 0.45406900 & 6.50174500 & 0.60501700 \\
\hline $\mathrm{C}$ & 0.83233600 & -5.23494000 & -2.04621900 \\
\hline $\mathrm{H}$ & 1.23786000 & -6.23723400 & -2.14643400 \\
\hline $\mathrm{C}$ & -4.88051600 & -1.64511600 & 4.27424100 \\
\hline $\mathrm{H}$ & -5.38722000 & -2.07569300 & 5.13286500 \\
\hline $\mathrm{C}$ & -2.82221300 & 0.96569600 & $\begin{array}{c}-4.55071100 \\
\text { S49 }\end{array}$ \\
\hline
\end{tabular}




$\begin{array}{lrrr}\mathrm{H} & -3.47561000 & 1.82862500 & -4.64432500 \\ \mathrm{C} & 3.97838300 & -1.16505000 & -0.37939300 \\ \mathrm{H} & 3.68484900 & -0.27021900 & -0.92144500 \\ \mathrm{C} & -0.07142600 & -2.79655800 & 4.25313200 \\ \mathrm{H} & -0.19764500 & -2.59257900 & 5.31255400 \\ \mathrm{C} & -0.51076300 & -4.00277500 & 3.71490700 \\ \mathrm{H} & -0.97755300 & -4.74749700 & 4.35342100 \\ \mathrm{C} & -0.33838200 & -4.25365100 & 2.35517600 \\ \mathrm{H} & -0.66570300 & -5.19600700 & 1.92411100 \\ \mathrm{C} & 1.15060300 & 3.47017000 & 2.91172400 \\ \mathrm{H} & 1.34522800 & 2.62902500 & 3.57185400 \\ \mathrm{C} & 3.63516800 & 2.65851200 & -1.22536000 \\ \mathrm{H} & 2.74760000 & 2.74007900 & -1.84858800 \\ \mathrm{C} & 4.88180900 & 2.97678800 & -1.75436800 \\ \mathrm{H} & 4.95956900 & 3.31422200 & -2.78343800 \\ \mathrm{C} & -4.92441700 & 0.69673000 & -0.89537000 \\ \mathrm{H} & -5.08965200 & -0.37885500 & -0.90453300 \\ \mathrm{C} & 0.52981300 & -1.84079500 & 3.43728400 \\ \mathrm{H} & 0.86457400 & -0.90883000 & 3.88257700 \\ \mathrm{C} & 5.91544100 & 2.42925400 & 0.35509500 \\ \mathrm{H} & 6.80314900 & 2.34177200 & 0.97440800 \\ \mathrm{C} & 1.68216200 & -4.13291300 & -2.05787800 \\ \mathrm{H} & 2.75493000 & -4.26666000 & -2.16666800 \\ \mathrm{H} & -2.35324900 & -1.27988500 & -0.43320100 \\ \mathrm{H} & -3.23636300 & -1.58723200 & -1.00217600 \\ \mathrm{H} & -1.12268300 & 2.08587300 & 1.39044200 \\ \mathrm{H} & 2.08817700 & 0.52412400 & 2.03919200 \\ \mathrm{H} & -32674000 & 0.70285600 & 2.80040600 \\ \mathrm{H} & -2.071900000 & -2.10882400 & 0.23212900 \\ \mathrm{H} & 0.28964800 & 2.08128700\end{array}$

\section{$5 \mathbf{b}$}

$\mathrm{Fe}$

$-0.18726800 \quad-1.29914000 \quad 0.59098900$ 


\begin{tabular}{|c|c|c|c|}
\hline $\mathrm{P}$ & 1.47405300 & -1.56914500 & -0.89113100 \\
\hline $\mathrm{P}$ & -2.18206900 & -0.65027900 & 1.19151900 \\
\hline 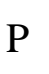 & -1.06004500 & 1.83326900 & 0.19348600 \\
\hline$P$ & 2.10137200 & 1.08873500 & -0.09933000 \\
\hline 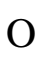 & 1.18474700 & -1.35872400 & 3.18071200 \\
\hline O & -1.05226600 & -4.07930700 & 0.34237300 \\
\hline $\mathrm{C}$ & 0.64838200 & -1.36017900 & 2.14441000 \\
\hline$C$ & 2.74908400 & -3.52316800 & 0.75979100 \\
\hline $\mathrm{H}$ & 1.85680900 & -3.48902800 & 1.37952100 \\
\hline$C$ & -1.78863200 & 1.66426100 & -1.45534300 \\
\hline 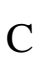 & 4.97163700 & -4.37582000 & 0.34947600 \\
\hline $\mathrm{H}$ & 5.81232100 & -4.99699500 & 0.64704200 \\
\hline 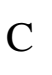 & -2.87088800 & -1.19526200 & 2.80369300 \\
\hline $\mathrm{C}$ & 2.28978400 & 2.76694100 & -0.81674200 \\
\hline$c$ & -3.54221500 & -1.00487700 & -0.00556200 \\
\hline 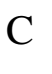 & -0.70007100 & -2.96514000 & 0.41179100 \\
\hline $\mathrm{C}$ & 3.96222300 & -2.82740200 & -1.19667500 \\
\hline $\mathrm{H}$ & 4.01955700 & -2.24038300 & -2.11276000 \\
\hline$C$ & 3.27898400 & 1.60930800 & 2.44040200 \\
\hline $\mathrm{H}$ & 2.41711400 & 2.24326900 & 2.64358000 \\
\hline$C$ & -0.93925700 & 3.62422000 & 0.51468600 \\
\hline $\mathrm{C}$ & 1.95343300 & 2.95372500 & -2.16352100 \\
\hline $\mathrm{H}$ & 1.67935500 & 2.08496300 & -2.75869700 \\
\hline B & 0.42730500 & 0.79687400 & 0.78423500 \\
\hline$C$ & 2.80886500 & -2.75282300 & -0.40239700 \\
\hline $\mathrm{C}$ & 0.84172300 & -1.61939400 & -3.64292900 \\
\hline $\mathrm{H}$ & 1.14790600 & -0.57435700 & -3.62905800 \\
\hline 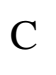 & -3.65701400 & 1.98666300 & -2.95711800 \\
\hline $\mathrm{H}$ & -4.66667500 & 2.34485100 & -3.13742100 \\
\hline $\mathrm{C}$ & 2.65952800 & 3.88101700 & -0.05377900 \\
\hline$\Pi$ & 2.93859800 & 3.75523100 & 0.98981100 \\
\hline 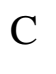 & 0.58274500 & -3.71704800 & -2.49294400 \\
\hline $\mathrm{H}$ & 0.67612700 & -4.32004500 & -1.59307000 \\
\hline $\mathrm{C}$ & 0.94026500 & -2.36297800 & -2.46396700 \\
\hline$C$ & -3.84118400 & -2.12362000 & 5.26505400 \\
\hline $\mathrm{H}$ & -4.21493600 & -2.48198400 & 6.22011600 \\
\hline
\end{tabular}




\begin{tabular}{|c|c|c|c|}
\hline $\mathrm{C}$ & -2.45656500 & -2.43071600 & 3.31340300 \\
\hline $\mathrm{H}$ & -1.73500600 & -3.02242000 & 2.75650900 \\
\hline $\mathrm{C}$ & 3.82481300 & -4.32830900 & 1.13485500 \\
\hline $\mathrm{H}$ & 3.76512600 & -4.91521800 & 2.04770500 \\
\hline $\mathrm{C}$ & 3.39403100 & 0.96853400 & 1.19992800 \\
\hline $\mathrm{C}$ & -4.24926200 & -0.88525600 & 4.77729000 \\
\hline $\mathrm{H}$ & -4.94049200 & -0.27290800 & 5.35021200 \\
\hline $\mathrm{C}$ & 5.42821200 & -0.09834400 & 1.97396200 \\
\hline $\mathrm{H}$ & 6.25332700 & -0.78241000 & 1.79247500 \\
\hline $\mathrm{C}$ & 5.31193800 & 0.55387300 & 3.19818800 \\
\hline $\mathrm{H}$ & 6.04947500 & 0.38589900 & 3.97837800 \\
\hline $\mathrm{C}$ & 0.37478900 & -2.20614600 & -4.81773100 \\
\hline $\mathrm{H}$ & 0.31460900 & -1.61567400 & -5.72969600 \\
\hline $\mathrm{C}$ & -3.08448000 & 2.13554000 & -1.69879500 \\
\hline $\mathrm{H}$ & -3.65104100 & 2.61901900 & -0.90437700 \\
\hline $\mathrm{C}$ & 1.98417600 & 4.22366300 & -2.73250600 \\
\hline $\mathrm{H}$ & 1.72959300 & 4.35044700 & -3.78184800 \\
\hline $\mathrm{C}$ & 2.68608800 & 5.14995500 & -0.62323000 \\
\hline $\mathrm{H}$ & 2.97089600 & 6.00568300 & -0.01657100 \\
\hline $\mathrm{C}$ & -2.93931700 & 1.36559100 & -3.97781800 \\
\hline $\mathrm{H}$ & -3.39232700 & 1.23828800 & -4.95735500 \\
\hline $\mathrm{C}$ & -5.59488600 & -1.40626300 & -1.87471400 \\
\hline $\mathrm{H}$ & -6.38957700 & -1.55835200 & -2.60029800 \\
\hline $\mathrm{C}$ & 4.23694900 & 1.40965200 & 3.42932900 \\
\hline $\mathrm{H}$ & 4.13368800 & 1.90664200 & 4.39037900 \\
\hline $\mathrm{C}$ & -1.08953400 & 4.58336000 & -0.49165900 \\
\hline $\mathrm{H}$ & -1.35365000 & 4.27531500 & -1.50031400 \\
\hline $\mathrm{C}$ & -3.26246000 & -1.54335500 & -1.26539300 \\
\hline $\mathrm{H}$ & -2.23633900 & -1.79050200 & -1.52554400 \\
\hline $\mathrm{C}$ & -2.94408000 & -2.89447600 & 4.53112100 \\
\hline $\mathrm{H}$ & -2.61076100 & -3.85540000 & 4.91241700 \\
\hline $\mathrm{C}$ & -0.00233200 & -3.54570300 & -4.82867700 \\
\hline $\mathrm{H}$ & -0.36956200 & -4.00332400 & -5.74364300 \\
\hline $\mathrm{C}$ & 2.34766200 & 5.32533200 & -1.96324100 \\
\hline $\mathrm{H}$ & 2.37117700 & 6.31788100 & -2.40606900 \\
\hline $\mathrm{C}$ & 5.03583400 & -3.62668000 & $\begin{array}{c}-0.82531600 \\
\text { S52 }\end{array}$ \\
\hline
\end{tabular}




\begin{tabular}{|c|c|c|c|}
\hline $\mathrm{H}$ & 5.92448600 & -3.66676900 & -1.45022900 \\
\hline $\mathrm{C}$ & -1.06958500 & 1.05016500 & -2.48414200 \\
\hline H & -0.07002500 & 0.66587700 & -2.27601400 \\
\hline $\mathrm{C}$ & -0.37772800 & 5.38537400 & 2.08151200 \\
\hline $\mathrm{H}$ & -0.09781300 & 5.69408600 & 3.08494300 \\
\hline $\mathrm{C}$ & -0.53645400 & 6.33601300 & 1.07429300 \\
\hline $\mathrm{H}$ & -0.37871100 & 7.38911900 & 1.29038300 \\
\hline $\mathrm{C}$ & -0.88783900 & 5.93220600 & -0.20955400 \\
\hline $\mathrm{H}$ & -0.99821700 & 6.66741200 & -1.00205200 \\
\hline $\mathrm{C}$ & -3.76707400 & -0.42382600 & 3.55560500 \\
\hline $\mathrm{H}$ & -4.08976400 & 0.54992700 & 3.19176100 \\
\hline $\mathrm{C}$ & -4.87015900 & -0.68681900 & 0.30998200 \\
\hline $\mathrm{H}$ & -5.11479600 & -0.28936800 & 1.29360500 \\
\hline $\mathrm{C}$ & -5.88833800 & -0.88319500 & -0.61677000 \\
\hline $\mathrm{H}$ & -6.91258000 & -0.63138400 & -0.35490700 \\
\hline $\mathrm{C}$ & 4.47450900 & 0.10964500 & 0.98207100 \\
\hline $\mathrm{H}$ & 4.54789800 & -0.41880900 & 0.03382200 \\
\hline $\mathrm{C}$ & -0.57200200 & 4.03843400 & 1.80361700 \\
\hline $\mathrm{H}$ & -0.42153400 & 3.29629000 & 2.58619700 \\
\hline $\mathrm{C}$ & -4.28240400 & -1.73822000 & -2.19329900 \\
\hline $\mathrm{H}$ & -4.04022800 & -2.14813400 & -3.17063900 \\
\hline $\mathrm{C}$ & 0.10888900 & -4.30118200 & -3.66255700 \\
\hline $\mathrm{H}$ & -0.17039700 & -5.35138400 & -3.66474500 \\
\hline $\mathrm{C}$ & -1.64881600 & 0.90055000 & -3.74131000 \\
\hline $\mathrm{H}$ & -1.09240000 & 0.40003300 & -4.52934000 \\
\hline $\mathrm{H}$ & 0.48678300 & 1.21540200 & 1.92010200 \\
\hline $\mathrm{H}$ & -0.74513400 & -0.79556500 & -0.73524900 \\
\hline $\mathrm{C}$ & -2.33360300 & 1.19731100 & 1.33330000 \\
\hline $\mathrm{H}$ & -2.03240600 & 1.49066000 & 2.34466300 \\
\hline $\mathrm{H}$ & -3.33959700 & 1.57862400 & 1.13263100 \\
\hline $\mathrm{C}$ & 2.22558300 & -0.06292500 & -1.36038500 \\
\hline $\mathrm{H}$ & 3.09836800 & -0.06893500 & -2.01417000 \\
\hline
\end{tabular}

$\begin{array}{crrc}\text { 6b' } & & & \\ \mathrm{Fe} & -0.61731300 & 0.99519000 & -1.06510400 \\ \mathrm{P} & 0.42691800 & 1.85806800 & 0.64533000 \\ & & & \mathrm{~S} 53\end{array}$




\begin{tabular}{|c|c|c|c|}
\hline $\mathrm{P}$ & -2.36739900 & -0.26857600 & -0.88399100 \\
\hline $\mathrm{P}$ & -0.22993900 & -2.21028900 & -0.20254700 \\
\hline 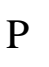 & 2.28454500 & -0.19310000 & -0.12999200 \\
\hline 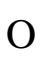 & 0.34196700 & 1.04108100 & -3.81250400 \\
\hline 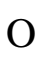 & -2.24036000 & 3.39175500 & -1.4357560 \\
\hline$C$ & -0.01509600 & 1.01550500 & -2.68819900 \\
\hline $\mathrm{C}$ & 2.12353600 & 4.07385600 & 1.30218700 \\
\hline $\mathrm{H}$ & 2.05509700 & 3.74894700 & 2.33912400 \\
\hline 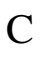 & -0.29330500 & -2.23480800 & 1.61940800 \\
\hline$C$ & 3.00484300 & 5.60941500 & -0.33651700 \\
\hline $\mathrm{H}$ & 3.62655800 & 6.46556900 & -0.58597000 \\
\hline $\mathrm{C}$ & -3.56215400 & -0.29140100 & -2.30054600 \\
\hline $\mathrm{C}$ & 3.46694200 & -1.45612900 & 0.46591900 \\
\hline $\mathrm{C}$ & -3.61626500 & -0.09494100 & 0.46580400 \\
\hline $\mathrm{C}$ & -1.58181900 & 2.43113300 & -1.27010000 \\
\hline 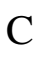 & 1.49544300 & 3.85148300 & -1.01057900 \\
\hline $\mathrm{H}$ & 0.95254700 & 3.30969200 & -1.78299300 \\
\hline $\mathrm{C}$ & 3.19339200 & 0.89916500 & -2.54234100 \\
\hline $\mathrm{H}$ & 2.46250200 & 0.25975800 & -3.02521400 \\
\hline$C$ & 0.29707100 & -3.90345700 & -0.64076300 \\
\hline $\mathrm{C}$ & 3.27396200 & -2.09625500 & 1.69656700 \\
\hline $\mathrm{H}$ & 2.50832200 & -1.74193700 & 2.38286300 \\
\hline $\mathrm{B}$ & 0.71037100 & -0.76756700 & -1.05159100 \\
\hline $\mathrm{C}$ & 1.40546000 & 3.39824500 & 0.30780300 \\
\hline$C$ & 0.19498900 & 2.26862200 & 3.48710400 \\
\hline $\mathrm{H}$ & 1.25112900 & 2.01844600 & 3.57139100 \\
\hline $\mathrm{C}$ & 0.19491900 & -3.28807900 & 3.74681000 \\
\hline $\mathrm{H}$ & 0.58408600 & -4.13597200 & 4.30449500 \\
\hline $\mathrm{C}$ & 4.47494300 & -1.92246100 & -0.38997600 \\
\hline $\mathrm{H}$ & 4.63925900 & -1.42522500 & -1.34441200 \\
\hline$C$ & -1.79293100 & 2.57875700 & 2.16933000 \\
\hline $\mathrm{H}$ & -2.29456600 & 2.56490500 & 1.20419700 \\
\hline $\mathrm{C}$ & -0.42423400 & 2.29139000 & 2.22959000 \\
\hline $\mathrm{C}$ & -5.33128400 & -0.34241100 & -4.47157300 \\
\hline $\mathrm{H}$ & -6.01890900 & -0.36021100 & -5.3132250 \\
\hline $\mathrm{C}$ & -3.28167200 & 0.43250700 & -3.46028100 \\
\hline
\end{tabular}




\begin{tabular}{|c|c|c|c|}
\hline $\mathrm{H}$ & -2.36603900 & 1.01490900 & -3.50836900 \\
\hline $\mathrm{C}$ & 2.92128500 & 5.16831900 & 0.98309400 \\
\hline $\mathrm{H}$ & 3.47289600 & 5.68266700 & 1.76623400 \\
\hline $\mathrm{C}$ & 3.30562900 & 0.90382500 & -1.14967000 \\
\hline $\mathrm{C}$ & -5.62166600 & -1.07022100 & -3.31801100 \\
\hline $\mathrm{H}$ & -6.53565700 & -1.65590200 & -3.25838000 \\
\hline $\mathrm{C}$ & 5.04097300 & 2.58282200 & -1.30523700 \\
\hline $\mathrm{H}$ & 5.74917900 & 3.24713300 & -0.81796100 \\
\hline $\mathrm{C}$ & 4.91223500 & 2.58712800 & -2.69255500 \\
\hline $\mathrm{H}$ & 5.52731400 & 3.25242300 & -3.29249800 \\
\hline $\mathrm{C}$ & -0.52876600 & 2.54384500 & 4.64556000 \\
\hline $\mathrm{H}$ & -0.03027800 & 2.51970900 & 5.61174800 \\
\hline $\mathrm{C}$ & 0.20066500 & -3.32211800 & 2.35566200 \\
\hline $\mathrm{H}$ & 0.60786700 & -4.18955500 & 1.84270000 \\
\hline $\mathrm{C}$ & 4.05365900 & -3.19223700 & 2.05243500 \\
\hline $\mathrm{H}$ & 3.88771100 & -3.67996500 & 3.00990300 \\
\hline $\mathrm{C}$ & 5.25615500 & -3.01561700 & -0.02899600 \\
\hline $\mathrm{H}$ & 6.03568500 & -3.36577700 & -0.70048900 \\
\hline $\mathrm{C}$ & -0.30607300 & -2.17634800 & 4.42191100 \\
\hline $\mathrm{H}$ & -0.31166100 & -2.15672800 & 5.50844500 \\
\hline $\mathrm{C}$ & -5.45556400 & 0.42692200 & 2.53532800 \\
\hline $\mathrm{H}$ & -6.16436200 & 0.62852200 & 3.33397800 \\
\hline $\mathrm{C}$ & 3.99311600 & 1.74282200 & -3.30856800 \\
\hline $\mathrm{H}$ & 3.88330000 & 1.74417200 & -4.38916500 \\
\hline $\mathrm{C}$ & 1.61334600 & -4.12413000 & -1.05669800 \\
\hline $\mathrm{H}$ & 2.29519900 & -3.28235100 & -1.14811100 \\
\hline $\mathrm{C}$ & -4.39642200 & 1.07420800 & 0.46350000 \\
\hline $\mathrm{H}$ & -4.28207300 & 1.79106100 & -0.34902100 \\
\hline $\mathrm{C}$ & -4.16304000 & 0.40978100 & -4.53914400 \\
\hline $\mathrm{H}$ & -3.93424900 & 0.98433400 & -5.43261800 \\
\hline $\mathrm{C}$ & -1.88842100 & 2.83566000 & 4.56677800 \\
\hline $\mathrm{H}$ & -2.45496700 & 3.04312600 & 5.47066900 \\
\hline $\mathrm{C}$ & 5.04245100 & -3.65828600 & 1.18913500 \\
\hline $\mathrm{H}$ & 5.64968500 & -4.51491300 & 1.46727500 \\
\hline $\mathrm{C}$ & 2.28738800 & 4.95121300 & -1.33067400 \\
\hline $\mathrm{H}$ & 2.35066400 & 5.28513500 & $\begin{array}{c}-2.36327900 \\
\text { S55 }\end{array}$ \\
\hline
\end{tabular}




\begin{tabular}{|c|c|c|c|}
\hline $\mathrm{C}$ & -0.79880000 & -1.12293800 & 2.30899400 \\
\hline $\mathrm{H}$ & -1.18139700 & -0.27269300 & 1.74678000 \\
\hline $\mathrm{C}$ & -0.14077400 & -6.27717000 & -0.85372900 \\
\hline $\mathrm{H}$ & -0.82840400 & -7.11499700 & -0.77892800 \\
\hline $\mathrm{C}$ & 1.17687400 & -6.48725500 & -1.25743400 \\
\hline $\mathrm{H}$ & 1.51705700 & -7.49096600 & -1.49713500 \\
\hline $\mathrm{C}$ & 2.05257500 & -5.41043700 & -1.35692100 \\
\hline $\mathrm{H}$ & 3.08101700 & -5.56630300 & -1.67210900 \\
\hline $\mathrm{C}$ & -4.74600700 & -1.03976100 & -2.23887100 \\
\hline $\mathrm{H}$ & -4.98751200 & -1.58826700 & -1.32895800 \\
\hline $\mathrm{C}$ & -3.78626200 & -0.99414600 & 1.52374100 \\
\hline $\mathrm{H}$ & -3.20591400 & -1.91231000 & 1.56330600 \\
\hline $\mathrm{C}$ & -4.68858400 & -0.73205400 & 2.55379500 \\
\hline $\mathrm{H}$ & -4.79524400 & -1.44607300 & 3.36704600 \\
\hline $\mathrm{C}$ & 4.24498300 & 1.74244400 & -0.53652600 \\
\hline $\mathrm{H}$ & 4.34689300 & 1.75602200 & 0.54651900 \\
\hline $\mathrm{C}$ & -0.57766600 & -4.99418700 & -0.54312000 \\
\hline $\mathrm{H}$ & -1.60346200 & -4.84058200 & -0.21413700 \\
\hline $\mathrm{C}$ & -5.30916400 & 1.32773700 & 1.47928500 \\
\hline $\mathrm{H}$ & -5.90847800 & 2.23435900 & 1.44868200 \\
\hline $\mathrm{C}$ & -2.51886700 & 2.85027300 & 3.32464400 \\
\hline $\mathrm{H}$ & -3.58342400 & 3.05822600 & 3.25183800 \\
\hline $\mathrm{C}$ & -0.80579300 & -1.09512000 & 3.70068200 \\
\hline $\mathrm{H}$ & -1.20924600 & -0.22248300 & 4.20936900 \\
\hline $\mathrm{H}$ & 1.06990400 & -1.24642000 & -2.10515300 \\
\hline $\mathrm{C}$ & -1.93969600 & -2.08858100 & -0.82246500 \\
\hline $\mathrm{H}$ & -1.90722300 & -2.43050200 & -1.86337900 \\
\hline $\mathrm{H}$ & -2.65131700 & -2.72378800 & -0.28441700 \\
\hline $\mathrm{C}$ & 1.79117200 & 0.77544700 & 1.31720200 \\
\hline $\mathrm{H}$ & 2.62717700 & 1.31375300 & 1.77568700 \\
\hline $\mathrm{H}$ & 1.36077500 & 0.09785500 & 2.06174100 \\
\hline
\end{tabular}

\section{$6 b$}

$\begin{array}{crrc}\mathrm{Fe} & -0.58733900 & 1.39708700 & -0.13425700 \\ \mathrm{P} & -2.31586600 & 0.13499800 & -0.46746100 \\ \mathrm{P} & 1.32387200 & 2.15558800 & 0.48021300 \\ & & & \mathrm{~S} 56\end{array}$




\begin{tabular}{|c|c|c|c|}
\hline $\mathrm{P}$ & 2.01834500 & -0.32439600 & -0.89418500 \\
\hline D & -0.50013400 & -1.95371000 & 0.30926000 \\
\hline $\mathrm{O}$ & -1.99520800 & 3.36693200 & 1.50341700 \\
\hline $\mathrm{C}$ & -4.84071900 & -0.70335700 & 0.54635400 \\
\hline $\mathrm{H}$ & -5.07741300 & -0.98079400 & -0.4799940 \\
\hline $\mathrm{C}$ & 1.78152200 & -0.45507000 & -2.68745900 \\
\hline$C$ & -5.47857300 & -0.57505500 & 2.86915900 \\
\hline $\mathrm{H}$ & -6.20338300 & -0.75970700 & 3.65790000 \\
\hline $\mathrm{C}$ & 1.92084300 & 1.92903200 & 2.21004200 \\
\hline $\mathrm{C}$ & 0.28282900 & -3.52433800 & -0.19471300 \\
\hline $\mathrm{C}$ & 1.68547600 & 3.93969800 & 0.21979900 \\
\hline $\mathrm{C}$ & -1.44162900 & 2.53723800 & 0.87573400 \\
\hline $\mathrm{C}$ & -3.33497000 & 0.27087000 & 2.15403600 \\
\hline $\mathrm{H}$ & -2.37165100 & 0.72824900 & 2.37308400 \\
\hline $\mathrm{C}$ & -0.65359900 & -1.65948100 & 3.09156700 \\
\hline $\mathrm{H}$ & 0.18502100 & -0.97672300 & 2.98542400 \\
\hline$C$ & 3.37445800 & -1.48349900 & -0.49800600 \\
\hline $\mathrm{C}$ & 0.32913300 & -3.92194000 & -1.53769700 \\
\hline $\mathrm{H}$ & -0.19567200 & -3.34894900 & -2.29944900 \\
\hline B & 0.55125200 & -0.38285300 & 0.32242900 \\
\hline $\mathrm{C}$ & -3.61243600 & -0.09613700 & 0.83579300 \\
\hline$C$ & -3.83915700 & -0.43108500 & -2.84139100 \\
\hline $\mathrm{H}$ & -3.64427800 & -1.48970700 & -2.68119100 \\
\hline $\mathrm{C}$ & 2.70333300 & -0.56223600 & -4.92500500 \\
\hline $\mathrm{H}$ & 3.56605700 & -0.55623600 & -5.58516500 \\
\hline $\mathrm{C}$ & 0.99596300 & -4.26675900 & 0.75705000 \\
\hline $\mathrm{H}$ & 0.98267100 & -3.95676600 & 1.80036600 \\
\hline $\mathrm{C}$ & -3.64022800 & 1.87413900 & -2.17139800 \\
\hline $\mathrm{H}$ & -3.23743300 & 2.62211300 & -1.49041900 \\
\hline $\mathrm{C}$ & -3.35549300 & 0.52219500 & -1.93751400 \\
\hline C & 2.71854700 & 1.62160000 & 4.87683000 \\
\hline $\mathrm{H}$ & 3.02900400 & 1.50161900 & 5.91130600 \\
\hline $\mathrm{C}$ & 0.99591800 & 1.55772100 & 3.18847600 \\
\hline $\mathrm{H}$ & -0.03346600 & 1.37755000 & 2.87268000 \\
\hline$C$ & -5.76720500 & -0.94340300 & 1.55621600 \\
\hline $\mathrm{H}$ & -6.71896500 & -1.41134400 & $\begin{array}{c}1.31758000 \\
\text { S57 }\end{array}$ \\
\hline
\end{tabular}




\begin{tabular}{|c|c|c|c|}
\hline $\mathrm{C}$ & -1.19149900 & -2.27599600 & 1.95748200 \\
\hline $\mathrm{C}$ & 3.64850000 & 1.99667800 & 3.90946900 \\
\hline $\mathrm{H}$ & 4.68404300 & 2.17307300 & 4.18835200 \\
\hline $\mathrm{C}$ & -2.82904300 & -3.38373400 & 3.35508700 \\
\hline $\mathrm{H}$ & -3.68348300 & -4.04731900 & 3.45245300 \\
\hline $\mathrm{C}$ & -2.29149500 & -2.76277400 & 4.48013400 \\
\hline $\mathrm{H}$ & -2.72507200 & -2.94484000 & 5.45963400 \\
\hline $\mathrm{C}$ & -4.58564200 & -0.04300800 & -3.95317400 \\
\hline $\mathrm{H}$ & -4.94940900 & -0.79562000 & -4.64818700 \\
\hline $\mathrm{C}$ & 2.88665300 & -0.45192800 & -3.54980500 \\
\hline $\mathrm{H}$ & 3.89265300 & -0.37522200 & -3.14130500 \\
\hline $\mathrm{C}$ & 1.05048400 & -5.05154700 & -1.91364800 \\
\hline $\mathrm{H}$ & 1.06919500 & -5.35357300 & -2.95726600 \\
\hline $\mathrm{C}$ & 1.71725000 & -5.39239700 & 0.37704100 \\
\hline $\mathrm{H}$ & 2.26528000 & -5.95790500 & 1.12561500 \\
\hline $\mathrm{C}$ & 1.41892600 & -0.67449300 & -5.45337100 \\
\hline $\mathrm{H}$ & 1.27929000 & -0.75099200 & -6.52805900 \\
\hline $\mathrm{C}$ & 2.03586500 & 6.68488900 & -0.24205900 \\
\hline $\mathrm{H}$ & 2.17434900 & 7.74766000 & -0.42094700 \\
\hline $\mathrm{C}$ & -1.20171200 & -1.90597100 & 4.34676800 \\
\hline $\mathrm{H}$ & -0.77822500 & -1.42033000 & 5.22181300 \\
\hline $\mathrm{C}$ & 3.66340500 & -2.60833200 & -1.28073500 \\
\hline $\mathrm{H}$ & 3.16948800 & -2.74273000 & -2.23975900 \\
\hline $\mathrm{C}$ & 1.71436300 & 4.84482400 & 1.28706200 \\
\hline $\mathrm{H}$ & 1.59700100 & 4.48493600 & 2.30617200 \\
\hline $\mathrm{C}$ & 1.39013200 & 1.40692500 & 4.51490700 \\
\hline $\mathrm{H}$ & 0.65868100 & 1.12341500 & 5.26795300 \\
\hline $\mathrm{C}$ & -4.86749300 & 1.30217600 & -4.16883700 \\
\hline $\mathrm{H}$ & -5.44834400 & 1.60507000 & -5.03574800 \\
\hline $\mathrm{C}$ & 1.74169400 & -5.79161200 & -0.95781900 \\
\hline $\mathrm{H}$ & 2.30340100 & -6.67357500 & -1.25287800 \\
\hline $\mathrm{C}$ & -4.26201300 & 0.03304300 & 3.16530000 \\
\hline $\mathrm{H}$ & -4.02901700 & 0.31968500 & 4.18773000 \\
\hline $\mathrm{C}$ & 0.49775000 & -0.55317700 & -3.23051100 \\
\hline $\mathrm{H}$ & -0.35522900 & -0.46218900 & -2.56488400 \\
\hline $\mathrm{C}$ & 4.90018000 & -2.31460900 & $\begin{array}{c}1.19703100 \\
\text { S58 }\end{array}$ \\
\hline
\end{tabular}




$\begin{array}{llll}\mathrm{H} & 5.37672900 & -2.19709200 & 2.16646500 \\ \mathrm{C} & 5.19539500 & -3.42026900 & 0.40049600 \\ \mathrm{H} & 5.90422300 & -4.16765000 & 0.74593400 \\ \mathrm{C} & 4.57074300 & -3.56494400 & -0.83493700 \\ \mathrm{H} & 4.78179600 & -4.43175800 & -1.45602600 \\ \mathrm{C} & 3.24955900 & 2.15586300 & 2.58426200 \\ \mathrm{H} & 3.97670300 & 2.47812100 & 1.83984500 \\ \mathrm{C} & 1.81004400 & 4.43511800 & -1.08511300 \\ \mathrm{H} & 1.73382000 & 3.75950700 & -1.93404700 \\ \mathrm{C} & 1.99329600 & 5.79451600 & -1.31283300 \\ \mathrm{H} & 2.09254300 & 6.15978200 & -2.33138600 \\ \mathrm{C} & -2.27930900 & -3.14646200 & 2.10042000 \\ \mathrm{H} & -2.70598900 & -3.63640300 & 1.22755300 \\ \mathrm{C} & 3.99321600 & -1.35820700 & 0.75665600 \\ \mathrm{H} & 3.75186900 & -0.50991400 & 1.39864500 \\ \mathrm{C} & 1.88779900 & 6.20705100 & 1.05635100 \\ \mathrm{H} & 1.90586400 & 6.89573800 & 1.89678100 \\ \mathrm{C} & -4.39649900 & 2.25944600 & -3.27172000 \\ \mathrm{H} & -4.60639500 & 3.31230200 & -3.43796100 \\ \mathrm{H} & 0.31489600 & -0.66866200 & -4.60357200 \\ \mathrm{H} & -0.69324200 & -0.72575200 & -5.00547200 \\ \mathrm{H} & 1.21466200 & -0.47325200 & 1.33780900 \\ \mathrm{H} & 2.67934800 & 1.34457600 & -0.52023300 \\ \mathrm{H} & 3.66362100 & 1.30442300 & -0.04138600 \\ \mathrm{H} & 2.78456400 & 1.90177300 & -1.45574200 \\ \mathrm{H} & -1.92627300 & -1.67720000 & -0.77351400 \\ \mathrm{H} & -0.43960600 & 2.01254600 & -1.76087600 \\ \mathrm{H} & -1.61802300 & -1.81550100 & -1.81354600\end{array}$

\section{$5 b^{\prime}$}

$\begin{array}{llll}\text { Fe } & -1.10814100 & 0.78416500 & -0.54369900 \\ \text { P } & -0.05756800 & 2.17623500 & 0.78768400 \\ \text { P } & -2.06753100 & -1.10135200 & -1.10971100 \\ \text { P } & 0.46591400 & -2.03228000 & 0.06562700 \\ & & & \text { S59 }\end{array}$




\begin{tabular}{|c|c|c|c|}
\hline $\mathrm{P}$ & 2.22509600 & 0.49972400 & -0.09182700 \\
\hline $\mathrm{O}$ & -0.07925000 & 1.68890500 & -3.14308600 \\
\hline $\mathrm{O}$ & -3.64020000 & 2.24022000 & -0.37572800 \\
\hline $\mathrm{C}$ & -0.48437000 & 1.34279600 & -2.1039430 \\
\hline 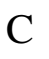 & -0.93820500 & 4.86543000 & 0.72289500 \\
\hline $\mathrm{H}$ & -1.61826000 & 4.59386600 & 1.52737100 \\
\hline $\mathrm{C}$ & -0.03142900 & -2.32278900 & 1.8327740 \\
\hline 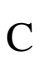 & -0.10410600 & 6.51275800 & -0.83267700 \\
\hline $\mathrm{H}$ & -0.12936400 & 7.51942400 & -1.2411030 \\
\hline$C$ & -2.65543500 & -1.32307400 & -2.8313830 \\
\hline $\mathrm{C}$ & 3.54532400 & -0.48191000 & 0.69507200 \\
\hline$c$ & -3.59202900 & -1.45227400 & -0.1406540 \\
\hline $\mathrm{C}$ & -2.62787800 & 1.66037200 & -0.4408770 \\
\hline$C$ & 0.81932900 & 4.28406300 & -0.82340000 \\
\hline $\mathrm{H}$ & 1.50661400 & 3.55209900 & -1.24643000 \\
\hline 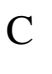 & 2.90051800 & 1.13366600 & -2.72651800 \\
\hline $\mathrm{H}$ & 2.15495000 & 0.37298700 & -2.95437500 \\
\hline$C$ & 1.76108100 & -3.28790900 & -0.23276800 \\
\hline$C$ & 3.51595000 & -0.79456400 & 2.05910600 \\
\hline $\mathrm{H}$ & 2.73366000 & -0.38665600 & 2.69613800 \\
\hline B & 0.72727200 & -0.37926600 & -0.61932100 \\
\hline $\mathrm{C}$ & -0.03565300 & 3.91999800 & 0.22530300 \\
\hline C & 0.00638500 & 3.06212800 & 3.46228500 \\
\hline $\mathrm{H}$ & 0.93533500 & 3.55781100 & 3.18352600 \\
\hline$C$ & -1.02771700 & -3.64935000 & 3.60127500 \\
\hline $\mathrm{H}$ & -1.43391800 & -4.59496700 & 3.95119400 \\
\hline $\mathrm{C}$ & 4.54375200 & -1.04588200 & -0.10857900 \\
\hline $\mathrm{H}$ & 4.57002900 & -0.81396300 & -1.17110000 \\
\hline $\mathrm{C}$ & -1.90758300 & 1.72088800 & 2.87012100 \\
\hline $\mathrm{H}$ & -2.45756400 & 1.14204900 & 2.13241800 \\
\hline$C$ & -0.70303400 & 2.32655600 & 2.50210700 \\
\hline $\mathrm{C}$ & -3.66375100 & -1.58336900 & -5.43150200 \\
\hline $\mathrm{H}$ & -4.04934800 & -1.68447300 & -6.44240900 \\
\hline $\mathrm{C}$ & -2.97375800 & -0.19026700 & -3.58653700 \\
\hline$\Pi$ & -2.82560100 & 0.79598400 & -3.15321200 \\
\hline $\mathrm{C}$ & -0.96944500 & 6.15417400 & 0.19635900 \\
\hline
\end{tabular}




\begin{tabular}{|c|c|c|c|}
\hline $\mathrm{H}$ & -1.67377500 & 6.87964500 & 0.59421900 \\
\hline $\mathrm{C}$ & 3.15376300 & 1.42491700 & -1.38619100 \\
\hline $\mathrm{C}$ & -3.35849200 & -2.71921600 & -4.68487500 \\
\hline $\mathrm{H}$ & -3.51000800 & -3.70808800 & -5.10917400 \\
\hline $\mathrm{C}$ & 4.79371400 & 3.07027000 & -2.07046300 \\
\hline $\mathrm{H}$ & 5.53815300 & 3.81970900 & -1.81416800 \\
\hline $\mathrm{C}$ & 4.51694400 & 2.78842000 & -3.40881600 \\
\hline $\mathrm{H}$ & 5.04045400 & 3.32598300 & -4.19497700 \\
\hline $\mathrm{C}$ & -0.47115500 & 3.17487300 & 4.76360900 \\
\hline $\mathrm{H}$ & 0.09052200 & 3.74540000 & 5.49848900 \\
\hline $\mathrm{C}$ & -0.56107500 & -3.53397000 & 2.29475900 \\
\hline $\mathrm{H}$ & -0.60707400 & -4.39549100 & 1.62932100 \\
\hline $\mathrm{C}$ & 4.47611100 & -1.63653300 & 2.61194100 \\
\hline $\mathrm{H}$ & 4.44692900 & -1.86234800 & 3.67447500 \\
\hline $\mathrm{C}$ & 5.49201700 & -1.90313100 & 0.44251400 \\
\hline $\mathrm{H}$ & 6.25099800 & -2.34775100 & -0.19580300 \\
\hline $\mathrm{C}$ & -0.98066700 & -2.55051800 & 4.45953100 \\
\hline $\mathrm{H}$ & -1.35941500 & -2.63896200 & 5.47455100 \\
\hline $\mathrm{C}$ & -5.87643200 & -1.87918000 & 1.43460300 \\
\hline $\mathrm{H}$ & -6.76193900 & -2.04300200 & 2.04291900 \\
\hline $\mathrm{C}$ & 3.57550000 & 1.81754100 & -3.73565300 \\
\hline $\mathrm{H}$ & 3.35829000 & 1.59611700 & -4.77704700 \\
\hline $\mathrm{C}$ & 2.36582300 & -4.03943700 & 0.77869400 \\
\hline $\mathrm{H}$ & 2.05318700 & -3.90878900 & 1.81183600 \\
\hline $\mathrm{C}$ & -4.85854300 & -1.52646600 & -0.72866400 \\
\hline $\mathrm{H}$ & -4.96819100 & -1.41757800 & -1.80455600 \\
\hline $\mathrm{C}$ & -3.47600000 & -0.31982300 & -4.87860400 \\
\hline $\mathrm{H}$ & -3.71541700 & 0.56936500 & -5.45475800 \\
\hline $\mathrm{C}$ & -1.67086800 & 2.56080000 & 5.12141500 \\
\hline $\mathrm{H}$ & -2.04427700 & 2.64935900 & 6.13824600 \\
\hline $\mathrm{C}$ & 5.46527300 & -2.19330400 & 1.80343900 \\
\hline $\mathrm{H}$ & 6.20895600 & -2.85900700 & 2.23347100 \\
\hline $\mathrm{C}$ & 0.79046800 & 5.57316600 & -1.34237500 \\
\hline $\mathrm{H}$ & 1.46494400 & 5.83969600 & -2.15198100 \\
\hline $\mathrm{C}$ & 0.01901200 & -1.23702100 & 2.70734100 \\
\hline $\mathrm{H}$ & 0.39972800 & -0.29079600 & $\begin{array}{c}2.32047100 \\
\text { S61 }\end{array}$ \\
\hline
\end{tabular}




\begin{tabular}{|c|c|c|c|}
\hline $\mathrm{C}$ & 3.21677800 & -4.34705300 & -1.85694100 \\
\hline $\mathrm{H}$ & 3.54834700 & -4.46250000 & -2.88563200 \\
\hline $\mathrm{C}$ & 3.81090800 & -5.09592500 & -0.84191300 \\
\hline $\mathrm{H}$ & 4.60749700 & -5.79729400 & -1.07665500 \\
\hline $\mathrm{C}$ & 3.38843200 & -4.93555500 & 0.47374400 \\
\hline $\mathrm{H}$ & 3.86327400 & -5.50026400 & 1.27212300 \\
\hline $\mathrm{C}$ & -2.86208900 & -2.58996300 & -3.39166100 \\
\hline $\mathrm{H}$ & -2.64649700 & -3.48370200 & -2.80954400 \\
\hline $\mathrm{C}$ & -3.48893000 & -1.58525900 & 1.25129200 \\
\hline $\mathrm{H}$ & -2.51652900 & -1.50195400 & 1.73077600 \\
\hline $\mathrm{C}$ & -4.61803600 & -1.80625200 & 2.03021500 \\
\hline $\mathrm{H}$ & -4.50990000 & -1.91497000 & 3.10712600 \\
\hline $\mathrm{C}$ & 4.11537100 & 2.39159300 & -1.06475700 \\
\hline $\mathrm{H}$ & 4.33955800 & 2.61093800 & -0.02158400 \\
\hline $\mathrm{C}$ & 2.20462300 & -3.44456900 & -1.55384900 \\
\hline $\mathrm{H}$ & 1.76319900 & -2.82298500 & -2.33229600 \\
\hline $\mathrm{C}$ & -5.99163800 & -1.73420900 & 0.05638200 \\
\hline $\mathrm{H}$ & -6.96883900 & -1.78288800 & -0.41685800 \\
\hline $\mathrm{C}$ & -2.38841100 & 1.83907700 & 4.17255700 \\
\hline $\mathrm{H}$ & -3.32448200 & 1.35650700 & 4.44259800 \\
\hline $\mathrm{C}$ & -0.45290000 & -1.34125400 & 4.01311900 \\
\hline $\mathrm{H}$ & -0.42557900 & -0.47334500 & 4.66837500 \\
\hline $\mathrm{H}$ & -1.31393100 & 0.03046000 & 0.77339900 \\
\hline $\mathrm{C}$ & -1.04514500 & -2.62320900 & -0.80736700 \\
\hline $\mathrm{H}$ & -0.71576700 & -3.02404900 & -1.77085600 \\
\hline $\mathrm{H}$ & -1.61167900 & -3.39716700 & -0.27836500 \\
\hline $\mathrm{C}$ & 1.75999500 & 1.85558700 & 1.08047600 \\
\hline $\mathrm{H}$ & 2.34213000 & 2.78102700 & 0.99288300 \\
\hline $\mathrm{H}$ & 1.87907800 & 1.47967300 & 2.10190200 \\
\hline
\end{tabular}

\section{8b}

$\begin{array}{lrrr}\mathrm{Fe} & 0.04531900 & -1.08918200 & -1.26529400 \\ \mathrm{P} & 2.22689600 & -0.76281600 & -1.35281200 \\ \mathrm{P} & -1.98459300 & -1.37390900 & -0.41754800 \\ \mathrm{P} & -1.56230800 & 1.28999800 & 0.66487600 \\ \mathrm{P} & 1.42149800 & 1.92684100 & -0.45449400\end{array}$




\begin{tabular}{|c|c|c|c|}
\hline $\mathrm{O}$ & 0.33159100 & -3.97106500 & -1.68810700 \\
\hline $\mathrm{O}$ & -0.27170500 & 0.06462800 & -3.93846700 \\
\hline $\mathrm{C}$ & 0.21480300 & -2.82446200 & -1.53195100 \\
\hline $\mathrm{C}$ & 2.85929200 & -2.24855500 & 0.92276300 \\
\hline $\mathrm{H}$ & 1.91581200 & -2.75723500 & 0.73399100 \\
\hline $\mathrm{C}$ & -3.05632300 & 2.17788200 & 0.10467300 \\
\hline $\mathrm{C}$ & 4.79315500 & -1.90579500 & 2.32625800 \\
\hline $\mathrm{H}$ & 5.37388300 & -2.16840100 & 3.20609400 \\
\hline $\mathrm{C}$ & -2.40898600 & -3.05641900 & 0.14013200 \\
\hline $\mathrm{C}$ & 1.48920800 & 3.65775900 & -1.01696300 \\
\hline $\mathrm{C}$ & -3.49196000 & -0.88777000 & -1.35012900 \\
\hline $\mathrm{C}$ & -0.18821300 & -0.43682900 & -2.88456600 \\
\hline $\mathrm{C}$ & 4.44882700 & -0.51367900 & 0.39185800 \\
\hline $\mathrm{H}$ & 4.77164600 & 0.32832700 & -0.21605200 \\
\hline $\mathrm{C}$ & 1.83358700 & 0.73074800 & 1.99344100 \\
\hline $\mathrm{H}$ & 1.09930800 & -0.00200400 & 1.65990200 \\
\hline $\mathrm{C}$ & -1.10138900 & 2.17729600 & 2.18048700 \\
\hline $\mathrm{C}$ & 2.70487100 & 4.27383800 & -1.34551600 \\
\hline $\mathrm{H}$ & 3.63880900 & 3.72436800 & -1.24635400 \\
\hline B & -0.26221000 & 1.06505000 & -0.74723500 \\
\hline $\mathrm{C}$ & 3.26736000 & -1.20379500 & 0.08581500 \\
\hline $\mathrm{C}$ & 4.54224200 & -1.34297300 & -2.86994300 \\
\hline $\mathrm{H}$ & 5.09652700 & -0.86251100 & -2.06768400 \\
\hline $\mathrm{C}$ & -5.19506300 & 3.15692100 & 0.68526000 \\
\hline $\mathrm{H}$ & -5.92762100 & 3.43039800 & 1.43945600 \\
\hline $\mathrm{C}$ & 0.29940900 & 4.37532800 & -1.18532900 \\
\hline $\mathrm{H}$ & -0.65420300 & 3.89019400 & -0.98087900 \\
\hline $\mathrm{C}$ & 2.46654500 & -2.12243700 & -3.81119800 \\
\hline $\mathrm{H}$ & 1.38934500 & -2.24074400 & -3.75436600 \\
\hline $\mathrm{C}$ & 3.14940500 & -1.47025800 & -2.77970700 \\
\hline $\mathrm{C}$ & -2.91673100 & -5.68183100 & 0.93831000 \\
\hline $\mathrm{H}$ & -3.12084100 & -6.70297100 & 1.25031400 \\
\hline $\mathrm{C}$ & -1.82819800 & -3.55975300 & 1.30834100 \\
\hline $\mathrm{H}$ & -1.17507500 & -2.95656500 & 1.93670000 \\
\hline $\mathrm{C}$ & 3.62507400 & -2.60108600 & 2.03104300 \\
\hline $\mathrm{H}$ & 3.28368100 & -3.39715700 & $\begin{array}{c}2.68526600 \\
\text { S63 }\end{array}$ \\
\hline
\end{tabular}




\begin{tabular}{|c|c|c|c|}
\hline $\mathrm{C}$ & 2.16485600 & 1.83060900 & 1.19389000 \\
\hline $\mathrm{C}$ & -3.48083700 & -5.18933800 & -0.23710200 \\
\hline $\mathrm{H}$ & -4.12166500 & -5.82351200 & -0.84398100 \\
\hline $\mathrm{C}$ & 3.71191000 & 2.57372800 & 2.89326600 \\
\hline $\mathrm{H}$ & 4.43347600 & 3.30320200 & 3.25133200 \\
\hline $\mathrm{C}$ & 3.39637400 & 1.45889600 & 3.66907200 \\
\hline $\mathrm{H}$ & 3.88153000 & 1.31473900 & 4.63109700 \\
\hline $\mathrm{C}$ & 5.23076900 & -1.84581800 & -3.96741200 \\
\hline $\mathrm{H}$ & 6.31091100 & -1.74107500 & -4.02013800 \\
\hline $\mathrm{C}$ & -4.01138100 & 2.53754300 & 1.06661400 \\
\hline $\mathrm{H}$ & -3.82066000 & 2.33586300 & 2.11893500 \\
\hline $\mathrm{C}$ & 2.72926400 & 5.58443400 & -1.80916900 \\
\hline $\mathrm{H}$ & 3.67762700 & 6.04941900 & -2.06297900 \\
\hline $\mathrm{C}$ & 0.32579200 & 5.68747100 & -1.64721400 \\
\hline $\mathrm{H}$ & -0.60578000 & 6.23106100 & -1.77642800 \\
\hline $\mathrm{C}$ & -5.43891000 & 3.42603500 & -0.66038000 \\
\hline $\mathrm{H}$ & -6.36588300 & 3.90817400 & -0.95885300 \\
\hline $\mathrm{C}$ & -5.80188200 & -0.16780900 & -2.76676100 \\
\hline $\mathrm{H}$ & -6.69604700 & 0.11867100 & -3.31390500 \\
\hline $\mathrm{C}$ & 2.45186200 & 0.53886900 & 3.22392100 \\
\hline $\mathrm{H}$ & 2.14857400 & -0.31830700 & 3.82392200 \\
\hline $\mathrm{C}$ & -0.96261000 & 3.57179300 & 2.11362400 \\
\hline $\mathrm{H}$ & -1.20104900 & 4.10059500 & 1.19250300 \\
\hline $\mathrm{C}$ & -3.44783200 & -0.69247700 & -2.73190700 \\
\hline $\mathrm{H}$ & -2.51154100 & -0.82445200 & -3.26349600 \\
\hline $\mathrm{C}$ & -2.09582800 & -4.86408000 & 1.71049200 \\
\hline $\mathrm{H}$ & -1.65280400 & -5.20427900 & 2.64334100 \\
\hline $\mathrm{C}$ & 4.53897000 & -2.48998500 & -4.99098200 \\
\hline $\mathrm{H}$ & 5.07805900 & -2.88805600 & -5.84626900 \\
\hline $\mathrm{C}$ & 1.53968400 & 6.29420700 & -1.95663200 \\
\hline $\mathrm{H}$ & 1.55926500 & 7.31645500 & -2.32335800 \\
\hline $\mathrm{C}$ & 5.20324600 & -0.85771600 & 1.50664800 \\
\hline $\mathrm{H}$ & 6.10031700 & -0.29238000 & 1.74457200 \\
\hline $\mathrm{C}$ & -3.30646600 & 2.45537900 & -1.24238700 \\
\hline $\mathrm{H}$ & -2.58375800 & 2.16247200 & -1.99959500 \\
\hline $\mathrm{C}$ & -0.41040400 & 2.23402100 & $\begin{array}{c}4.49020200 \\
\text { S64 }\end{array}$ \\
\hline
\end{tabular}




\begin{tabular}{|c|c|c|c|}
\hline $\mathrm{H}$ & -0.18898000 & 1.69569300 & 5.40762000 \\
\hline $\mathrm{C}$ & -0.25773800 & 3.61437200 & 4.41712000 \\
\hline $\mathrm{H}$ & 0.07738700 & 4.17281400 & 5.28695300 \\
\hline $\mathrm{C}$ & -0.53933800 & 4.28549200 & 3.22703800 \\
\hline $\mathrm{H}$ & -0.43216200 & 5.36486900 & 3.16690200 \\
\hline $\mathrm{C}$ & -3.23388000 & -3.87963200 & -0.63626900 \\
\hline $\mathrm{H}$ & -3.68698600 & -3.49814400 & -1.54900400 \\
\hline $\mathrm{C}$ & -4.72027300 & -0.74238200 & -0.68888200 \\
\hline $\mathrm{H}$ & -4.78807100 & -0.92390600 & 0.38171700 \\
\hline $\mathrm{C}$ & -5.86367900 & -0.37970900 & -1.39009300 \\
\hline $\mathrm{H}$ & -6.80587800 & -0.26209000 & -0.86131100 \\
\hline $\mathrm{C}$ & 3.10113100 & 2.76400000 & 1.65753900 \\
\hline $\mathrm{H}$ & 3.34026200 & 3.64505800 & 1.06758800 \\
\hline $\mathrm{C}$ & -0.82720200 & 1.50822200 & 3.37590000 \\
\hline $\mathrm{H}$ & -0.91575400 & 0.42541500 & 3.46001100 \\
\hline $\mathrm{C}$ & -4.59491100 & -0.33327300 & -3.43701400 \\
\hline $\mathrm{H}$ & -4.54028000 & -0.18405400 & -4.51190800 \\
\hline $\mathrm{C}$ & 3.15778200 & -2.62870400 & -4.90900100 \\
\hline $\mathrm{H}$ & 2.61156700 & -3.13674800 & -5.69837000 \\
\hline $\mathrm{C}$ & -4.49445200 & 3.07667000 & -1.62016200 \\
\hline $\mathrm{H}$ & -4.68363500 & 3.27435400 & -2.67168200 \\
\hline $\mathrm{H}$ & -0.68136700 & 1.68352300 & -1.69265700 \\
\hline $\mathrm{H}$ & 0.50868600 & -1.29189700 & 4.70985200 \\
\hline $\mathrm{H}$ & -1.33822900 & -1.39795600 & 3.91809200 \\
\hline $\mathrm{H}$ & 0.26077900 & -1.29875900 & 0.24219200 \\
\hline $\mathrm{H}$ & 0.28954900 & -1.67086900 & 2.74418400 \\
\hline $\mathrm{N}$ & -0.29689500 & -3.39220500 & 4.11348200 \\
\hline B & -0.19974600 & -1.87668000 & 3.87122900 \\
\hline $\mathrm{C}$ & 2.54360500 & 1.04535200 & -1.59812700 \\
\hline $\mathrm{H}$ & 3.59064900 & 1.35335400 & -1.51303200 \\
\hline $\mathrm{H}$ & 2.19962200 & 1.27599700 & -2.61378400 \\
\hline $\mathrm{C}$ & -2.11601000 & -0.37424200 & 1.12611500 \\
\hline $\mathrm{H}$ & -3.10036200 & -0.37922000 & 1.60450600 \\
\hline $\mathrm{H}$ & -1.39164200 & -0.80475400 & 1.83713200 \\
\hline $\mathrm{C}$ & -0.80333700 & -3.71402700 & 5.42092800 \\
\hline $\mathrm{H}$ & -0.97228000 & -4.79857900 & $\begin{array}{c}5.53378500 \\
\text { S65 }\end{array}$ \\
\hline
\end{tabular}




$\begin{array}{lrrr}\mathrm{H} & -0.12960200 & -3.40602400 & 6.25108200 \\ \mathrm{H} & -1.75981700 & -3.20357500 & 5.58330500 \\ \mathrm{C} & 0.91341400 & -4.11115300 & 3.84606300 \\ \mathrm{H} & 0.79268000 & -5.19111100 & 4.03618600 \\ \mathrm{H} & 1.18851800 & -3.99210200 & 2.78813800 \\ \mathrm{H} & 1.79016300 & -3.77875500 & 4.45290000\end{array}$

\section{$6 \mathrm{~b}+\mathrm{Me}_{2} \mathrm{NH}-\mathrm{BH}_{3}$}

\begin{tabular}{|c|c|c|c|}
\hline 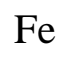 & 0.73293900 & -1.17461100 & -0.76773500 \\
\hline $\mathrm{P}$ & 2.50157700 & 0.09749800 & -0.96623000 \\
\hline D & -1.16552900 & -2.04976800 & -0.19254500 \\
\hline $\mathrm{P}$ & -2.10044600 & 0.60518500 & 0.46148500 \\
\hline $\mathrm{P}$ & 0.47129300 & 2.19651100 & -0.67126000 \\
\hline $\mathrm{O}$ & 2.31991200 & -3.22260100 & 0.56776000 \\
\hline $\mathrm{O}$ & 0.81702100 & -2.01529100 & -3.55957800 \\
\hline $\mathrm{C}$ & 1.65568400 & -2.36002000 & 0.08917700 \\
\hline$C$ & 4.51232100 & -0.73977000 & 0.76290400 \\
\hline $\mathrm{H}$ & 4.36065500 & -1.69874600 & 0.27237800 \\
\hline 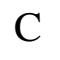 & -3.76531600 & 0.78707400 & -0.28539800 \\
\hline$C$ & 5.65458400 & 0.58217000 & 2.42962100 \\
\hline $\mathbf{H}$ & 6.38576900 & 0.66269800 & 3.22931200 \\
\hline $\mathrm{C}$ & -1.17592400 & -3.74242400 & 0.52433300 \\
\hline $\mathrm{C}$ & -0.10706300 & 3.70357000 & -1.53900300 \\
\hline$C$ & -2.55210600 & -2.19522400 & -1.41628500 \\
\hline 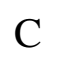 & 0.72695500 & -1.60661400 & -2.45831800 \\
\hline $\mathrm{C}$ & 3.98152600 & 1.59149000 & 1.01084400 \\
\hline $\mathrm{H}$ & 3.42658400 & 2.48006500 & 0.72741100 \\
\hline $\mathrm{C}$ & 0.89829600 & 1.75112400 & 2.03871600 \\
\hline 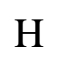 & 0.64841000 & 0.71271100 & 1.80455600 \\
\hline$C$ & -2.23018500 & 1.68388300 & 1.92931900 \\
\hline 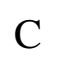 & 0.76740800 & 4.69422400 & -2.00720900 \\
\hline 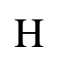 & 1.83805600 & 4.60037900 & -1.83644400 \\
\hline D & -0.62675700 & 0.66191200 & -0.79922600 \\
\hline $\mathrm{C}$ & 3.74931100 & 0.37087900 & 0.36935600 \\
\hline & 4.52979100 & 0.54707800 & -2.90820500 \\
\hline \multirow[t]{2}{*}{$\mathrm{H}$} & 4.46180500 & 1.60016200 & -2.64073500 \\
\hline & & & S66 \\
\hline
\end{tabular}




\begin{tabular}{|c|c|c|c|}
\hline $\mathrm{C}$ & -6.17004800 & 0.83231400 & 0.01247500 \\
\hline $\mathrm{H}$ & -7.03544800 & 0.76221100 & 0.66552600 \\
\hline $\mathrm{C}$ & -1.47526600 & 3.83862200 & -1.79893000 \\
\hline $\mathrm{H}$ & -2.15866300 & 3.05416600 & -1.47545000 \\
\hline $\mathrm{C}$ & 3.83855200 & -1.74144700 & -2.61338100 \\
\hline $\mathrm{H}$ & 3.19503000 & -2.47397600 & -2.13147200 \\
\hline $\mathrm{C}$ & 3.70224200 & -0.39073900 & -2.27986200 \\
\hline $\mathrm{C}$ & -1.10050400 & -6.37364900 & 1.49482800 \\
\hline $\mathrm{H}$ & -1.06890700 & -7.39241700 & 1.87186600 \\
\hline $\mathrm{C}$ & -1.88270700 & -4.09702100 & 1.67994000 \\
\hline $\mathrm{H}$ & -2.45005100 & -3.35111600 & 2.23057500 \\
\hline $\mathrm{C}$ & 5.45636400 & -0.63392900 & 1.77682300 \\
\hline $\mathrm{H}$ & 6.03740100 & -1.50754700 & 2.06098600 \\
\hline $\mathrm{C}$ & 0.89028500 & 2.71204800 & 1.01897000 \\
\hline $\mathrm{C}$ & -0.39049800 & -6.03042700 & 0.34512600 \\
\hline $\mathrm{H}$ & 0.19801200 & -6.78028100 & -0.17646300 \\
\hline $\mathrm{C}$ & 1.57151100 & 4.39051200 & 2.62399400 \\
\hline $\mathrm{H}$ & 1.82826500 & 5.42188000 & 2.85019100 \\
\hline $\mathrm{C}$ & 1.57785700 & 3.42914600 & 3.63167600 \\
\hline $\mathrm{H}$ & 1.84572300 & 3.70815700 & 4.64723100 \\
\hline $\mathrm{C}$ & 5.45388300 & 0.14697200 & -3.86822000 \\
\hline $\mathrm{H}$ & 6.08200400 & 0.88785100 & -4.35637100 \\
\hline $\mathrm{C}$ & -4.89303000 & 0.69992100 & 0.54340000 \\
\hline $\mathrm{H}$ & -4.76727000 & 0.53805600 & 1.61283400 \\
\hline $\mathrm{C}$ & 0.27995300 & 5.79911100 & -2.69843700 \\
\hline $\mathrm{H}$ & 0.96962300 & 6.55749100 & -3.05829500 \\
\hline $\mathrm{C}$ & -1.96431800 & 4.94551000 & -2.48554400 \\
\hline $\mathrm{H}$ & -3.03005900 & 5.03140300 & -2.67873900 \\
\hline $\mathrm{C}$ & -6.33840400 & 1.06126600 & -1.35242200 \\
\hline $\mathrm{H}$ & -7.33703000 & 1.16561900 & -1.76752400 \\
\hline $\mathrm{C}$ & -4.69265800 & -2.38387000 & -3.21837600 \\
\hline $\mathrm{H}$ & -5.52270000 & -2.45564600 & -3.91669900 \\
\hline $\mathrm{C}$ & 1.23170700 & 2.11174900 & 3.33941300 \\
\hline $\mathrm{H}$ & 1.20500600 & 1.35318500 & 4.11839000 \\
\hline $\mathrm{C}$ & -2.18154500 & 3.07451900 & 1.76872900 \\
\hline $\mathrm{H}$ & -2.04671600 & 3.50219600 & 0.77613800 \\
\hline
\end{tabular}




\begin{tabular}{|c|c|c|c|}
\hline $\mathrm{C}$ & -2.43018900 & -1.70567200 & -2.71733700 \\
\hline $\mathrm{H}$ & -1.50277800 & -1.23172200 & -3.02059400 \\
\hline $\mathrm{C}$ & -1.84501000 & -5.40526300 & 2.16074200 \\
\hline $\mathrm{H}$ & -2.39430200 & -5.66092200 & 3.06328800 \\
\hline $\mathrm{C}$ & 5.57846100 & -1.20105100 & -4.19579700 \\
\hline $\mathrm{H}$ & 6.30148900 & -1.51510600 & -4.94393100 \\
\hline $\mathrm{C}$ & -1.08688000 & 5.92786200 & -2.93582700 \\
\hline $\mathrm{H}$ & -1.46474700 & 6.78861000 & -3.48048200 \\
\hline $\mathrm{C}$ & 4.91623000 & 1.69375200 & 2.03927000 \\
\hline $\mathrm{H}$ & 5.06184600 & 2.65236500 & 2.53165600 \\
\hline $\mathrm{C}$ & -3.94428800 & 1.01589600 & -1.65083200 \\
\hline $\mathrm{H}$ & -3.07753400 & 1.06613100 & -2.30375500 \\
\hline $\mathrm{C}$ & -2.48118300 & 2.00070700 & 4.31586400 \\
\hline $\mathrm{H}$ & -2.58897700 & 1.57139700 & 5.30764100 \\
\hline $\mathrm{C}$ & -2.40948100 & 3.37902000 & 4.14881700 \\
\hline $\mathrm{H}$ & -2.46203200 & 4.03678800 & 5.01197800 \\
\hline $\mathrm{C}$ & -2.26361500 & 3.91574300 & 2.87036300 \\
\hline $\mathrm{H}$ & -2.19923800 & 4.99143100 & 2.73124600 \\
\hline $\mathrm{C}$ & -0.42731700 & -4.72712700 & -0.13555900 \\
\hline $\mathrm{H}$ & 0.14145600 & -4.45391700 & -1.02292900 \\
\hline $\mathrm{C}$ & -3.76375700 & -2.78552900 & -1.03090500 \\
\hline $\mathrm{H}$ & -3.87137200 & -3.19117600 & -0.02538700 \\
\hline $\mathrm{C}$ & -4.82744400 & -2.87513000 & -1.92074200 \\
\hline $\mathrm{H}$ & -5.76019800 & -3.33523700 & -1.60450100 \\
\hline $\mathrm{C}$ & 1.21989800 & 4.03785900 & 1.32398700 \\
\hline $\mathrm{H}$ & 1.19012200 & 4.80152000 & 0.55047700 \\
\hline $\mathrm{C}$ & -2.39408100 & 1.15396600 & 3.21346300 \\
\hline $\mathrm{H}$ & -2.42147400 & 0.08064900 & 3.37405800 \\
\hline $\mathrm{C}$ & -3.49255500 & -1.80241100 & -3.61374800 \\
\hline $\mathrm{H}$ & -3.37958600 & -1.41527900 & -4.62346500 \\
\hline $\mathrm{C}$ & 4.77557600 & -2.14386500 & -3.56078800 \\
\hline $\mathrm{H}$ & 4.86859100 & -3.19724700 & -3.81070100 \\
\hline $\mathrm{C}$ & -5.22535000 & 1.15255000 & -2.18045000 \\
\hline $\mathrm{H}$ & -5.34867700 & 1.31798100 & -3.24748700 \\
\hline $\mathrm{H}$ & -1.14256700 & 0.74707800 & -1.88736200 \\
\hline $\mathrm{H}$ & 0.33356400 & -1.63006600 & $\begin{array}{c}5.28444800 \\
\text { S68 }\end{array}$ \\
\hline
\end{tabular}




$\begin{array}{lrrr}\mathrm{H} & -1.11879600 & -2.14245600 & 3.96906100 \\ \mathrm{H} & 0.73721500 & -2.23153400 & 2.24090400 \\ \mathrm{H} & -0.11366400 & -0.40577900 & 3.74174800 \\ \mathrm{~N} & 1.03973500 & -2.29233800 & 3.22086300 \\ \mathrm{~B} & -0.06910600 & -1.55086400 & 4.14360200 \\ \mathrm{C} & 2.04824400 & 1.81103000 & -1.50796900 \\ \mathrm{H} & 2.81551200 & 2.57654000 & -1.34658200 \\ \mathrm{H} & 1.83878700 & 1.75189000 & -2.58240900 \\ \mathrm{C} & -2.06655400 & -1.08972200 & 1.11895900 \\ \mathrm{H} & -3.06608500 & -1.45676500 & 1.38404500 \\ \mathrm{H} & -1.43167600 & -1.09595100 & 2.01442300 \\ \mathrm{C} & 1.18349400 & -3.72225700 & 3.55366200 \\ \mathrm{H} & 1.87005700 & -4.20715300 & 2.85492900 \\ \mathrm{H} & 1.55820800 & -3.79863600 & 4.57703800 \\ \mathrm{H} & 0.20325200 & -4.19659600 & 3.49780600 \\ \mathrm{C} & 2.34678900 & -1.60667200 & 3.27957400 \\ \mathrm{H} & 3.10608900 & -2.19004500 & 2.75485800 \\ \mathrm{H} & 2.26086800 & -0.62866700 & 2.79831800 \\ \mathrm{H} & 2.61914200 & -1.47429700 & 4.32962900\end{array}$

\section{TS1}

$\begin{array}{lrrr}\mathrm{Fe} & 0.43233000 & -1.45120500 & -0.49378400 \\ \mathrm{P} & 2.52412300 & -0.62095500 & -0.67643000 \\ \mathrm{P} & -1.65808400 & -1.83559500 & 0.07797300 \\ \mathrm{P} & -1.90758800 & 1.00176900 & 0.53114500 \\ \mathrm{P} & 0.92757100 & 1.89486800 & -0.79477100 \\ \mathrm{O} & 1.48945800 & -4.12633400 & 0.00330700 \\ \mathrm{O} & 0.42573500 & -1.36933200 & -3.42691500 \\ \mathrm{C} & 1.04363900 & -3.05632600 & -0.16629100 \\ \mathrm{C} & 4.53914300 & -1.54314900 & 0.99166700 \\ \mathrm{H} & 4.23967100 & -2.51350100 & 0.59754200 \\ \mathrm{C} & -3.48081000 & 1.51264600 & -0.26152400 \\ \mathrm{C} & 5.95461700 & -0.23365200 & 2.43885300 \\ \mathrm{H} & 6.74903200 & -0.17545900 & 3.17757500 \\ \mathrm{C} & -2.13836500 & -3.44028000 & 0.82071900 \\ \mathrm{C} & 0.64381300 & 3.30506900 & -1.91715100\end{array}$




\begin{tabular}{|c|c|c|c|}
\hline $\mathrm{C}$ & -3.04388800 & -1.71634000 & -1.15453000 \\
\hline $\mathrm{C}$ & 0.39125400 & -1.42472000 & -2.25497900 \\
\hline $\mathrm{C}$ & 4.30646100 & 0.85070300 & 1.04597700 \\
\hline $\mathrm{H}$ & 3.84251200 & 1.77306900 & 0.71111000 \\
\hline $\mathrm{C}$ & 1.33480200 & 1.81039800 & 1.93886400 \\
\hline $\mathrm{H}$ & 0.87262200 & 0.82191000 & 1.89047700 \\
\hline $\mathrm{C}$ & -1.75897100 & 2.23538200 & 1.85831200 \\
\hline $\mathrm{C}$ & 1.69609800 & 3.98387200 & -2.54607700 \\
\hline $\mathrm{H}$ & 2.72534300 & 3.68038200 & -2.36704900 \\
\hline B & -0.47358400 & 0.61172400 & -0.71241800 \\
\hline $\mathrm{C}$ & 3.88261200 & -0.38432900 & 0.54985100 \\
\hline $\mathrm{C}$ & 4.78363100 & -0.83187400 & -2.32293400 \\
\hline $\mathrm{H}$ & 5.08661100 & 0.09067600 & -1.82974600 \\
\hline $\mathrm{C}$ & -5.79091400 & 2.18950700 & 0.02142100 \\
\hline $\mathrm{H}$ & -6.62529500 & 2.42572000 & 0.67581200 \\
\hline $\mathrm{C}$ & -0.67106500 & 3.70539400 & -2.17940200 \\
\hline $\mathrm{H}$ & -1.49755700 & 3.16247600 & -1.72349600 \\
\hline $\mathrm{C}$ & 3.23423900 & -2.65632300 & -2.56872400 \\
\hline $\mathrm{H}$ & 2.30854800 & -3.15467000 & -2.30236600 \\
\hline $\mathrm{C}$ & 3.57864600 & -1.45026600 & -1.95611200 \\
\hline $\mathrm{C}$ & -2.87968500 & -5.95928500 & 1.78955200 \\
\hline $\mathrm{H}$ & -3.16160300 & -6.93707200 & 2.17058600 \\
\hline $\mathrm{C}$ & -2.88484400 & -3.55439200 & 1.99789100 \\
\hline $\mathrm{H}$ & -3.14507600 & -2.66970800 & 2.57435900 \\
\hline $\mathrm{C}$ & 5.56056500 & -1.47016000 & 1.92789000 \\
\hline $\mathrm{H}$ & 6.05022800 & -2.38095600 & 2.26121600 \\
\hline $\mathrm{C}$ & 1.46516000 & 2.59325900 & 0.78613800 \\
\hline $\mathrm{C}$ & -2.14029300 & -5.85392900 & 0.61353800 \\
\hline $\mathrm{H}$ & -1.84329500 & -6.74774700 & 0.07215500 \\
\hline $\mathrm{C}$ & 2.51032000 & 4.33508000 & 2.09440200 \\
\hline $\mathrm{H}$ & 2.95952600 & 5.32276800 & 2.15561500 \\
\hline $\mathrm{C}$ & 2.38768900 & 3.54758200 & 3.23760100 \\
\hline $\mathrm{H}$ & 2.74874300 & 3.91978800 & 4.19259700 \\
\hline $\mathrm{C}$ & 5.61094700 & -1.39294000 & -3.28682600 \\
\hline $\mathrm{H}$ & 6.53774400 & -0.89531100 & -3.56006900 \\
\hline $\mathrm{C}$ & -4.56724300 & 1.82452800 & $\begin{array}{c}0.56856600 \\
\text { S70 }\end{array}$ \\
\hline
\end{tabular}




\begin{tabular}{|c|c|c|c|}
\hline $\mathrm{H}$ & -4.44649500 & 1.78906300 & 1.65005300 \\
\hline $\mathrm{C}$ & 1.43583500 & 5.04744400 & -3.40462400 \\
\hline $\mathrm{H}$ & 2.25977700 & 5.56393700 & -3.88840400 \\
\hline $\mathrm{C}$ & -0.93072700 & 4.77158700 & -3.03461700 \\
\hline $\mathrm{H}$ & -1.95793200 & 5.06705000 & -3.22932700 \\
\hline $\mathrm{C}$ & -5.94341200 & 2.25989500 & -1.36241600 \\
\hline $\mathrm{H}$ & -6.89982500 & 2.54815900 & -1.79012700 \\
\hline $\mathrm{C}$ & -5.20652400 & -1.55592300 & -2.93918200 \\
\hline $\mathrm{H}$ & -6.04339500 & -1.49096900 & -3.62978200 \\
\hline $\mathrm{C}$ & 1.79514800 & 2.28977200 & 3.16070300 \\
\hline $\mathrm{H}$ & 1.66837400 & 1.66416200 & 4.04081000 \\
\hline $\mathrm{C}$ & -1.50253100 & 3.56927400 & 1.51086600 \\
\hline $\mathrm{H}$ & -1.38158300 & 3.84669900 & 0.46376300 \\
\hline $\mathrm{C}$ & -2.82770100 & -1.54141400 & -2.52132700 \\
\hline $\mathrm{H}$ & -1.81777100 & -1.45381300 & -2.90085400 \\
\hline $\mathrm{C}$ & -3.25176000 & -4.80895300 & 2.47829700 \\
\hline $\mathrm{H}$ & -3.81768000 & -4.88434000 & 3.40280200 \\
\hline $\mathrm{C}$ & 5.25575600 & -2.59746000 & -3.89100300 \\
\hline $\mathrm{H}$ & 5.90422900 & -3.04404000 & -4.63986200 \\
\hline $\mathrm{C}$ & 0.12277700 & 5.44376000 & -3.64822500 \\
\hline $\mathrm{H}$ & -0.07833400 & 6.27133100 & -4.32252900 \\
\hline $\mathrm{C}$ & 5.33122800 & 0.92364300 & 1.98895900 \\
\hline $\mathrm{H}$ & 5.63570300 & 1.89553800 & 2.36928100 \\
\hline $\mathrm{C}$ & -3.64355000 & 1.58442500 & -1.64694900 \\
\hline $\mathrm{H}$ & -2.81806000 & 1.32181100 & -2.30261000 \\
\hline $\mathrm{C}$ & -1.78721900 & 2.87027000 & 4.18757300 \\
\hline $\mathrm{H}$ & -1.88707900 & 2.58650900 & 5.23116200 \\
\hline $\mathrm{C}$ & -1.50733900 & 4.18608600 & 3.83882400 \\
\hline $\mathrm{H}$ & -1.39355500 & 4.94205100 & 4.61096200 \\
\hline $\mathrm{C}$ & -1.36945000 & 4.53760600 & 2.49569300 \\
\hline $\mathrm{H}$ & -1.14633100 & 5.56363400 & 2.21682400 \\
\hline $\mathrm{C}$ & -1.77430600 & -4.60279300 & 0.13173800 \\
\hline $\mathrm{H}$ & -1.19328000 & -4.52298800 & -0.78495800 \\
\hline $\mathrm{C}$ & -4.36585400 & -1.82116200 & -0.69626900 \\
\hline $\mathrm{H}$ & -4.56101000 & -1.98820100 & 0.36211200 \\
\hline $\mathrm{C}$ & -5.43704100 & -1.73507200 & $\begin{array}{c}-1.57586700 \\
\text { S71 }\end{array}$ \\
\hline
\end{tabular}




\begin{tabular}{|c|c|c|c|}
\hline $\mathrm{H}$ & -6.45322500 & -1.81026500 & -1.19764600 \\
\hline $\mathrm{C}$ & 2.04764700 & 3.86370000 & 0.86982900 \\
\hline $\mathrm{H}$ & 2.12919000 & 4.49000700 & -0.01538800 \\
\hline $\mathrm{C}$ & -1.91524500 & 1.89126700 & 3.20337700 \\
\hline $\mathrm{H}$ & -2.09004100 & 0.86153400 & 3.49981200 \\
\hline $\mathrm{C}$ & -3.90116600 & -1.46456700 & -3.40828700 \\
\hline $\mathrm{H}$ & -3.70952000 & -1.32891000 & -4.46970700 \\
\hline $\mathrm{C}$ & 4.07181800 & -3.22780500 & -3.52583300 \\
\hline $\mathrm{H}$ & 3.79068600 & -4.17070200 & -3.98668900 \\
\hline $\mathrm{C}$ & -4.86911100 & 1.95922400 & -2.19256700 \\
\hline $\mathrm{H}$ & -4.98307900 & 1.99973900 & -3.27254000 \\
\hline $\mathrm{H}$ & -0.99431100 & 0.69085700 & -1.79958200 \\
\hline $\mathrm{H}$ & 0.33374200 & -1.26574400 & 5.17070300 \\
\hline $\mathrm{H}$ & -1.33043700 & -1.15521400 & 4.05701900 \\
\hline $\mathrm{H}$ & 0.37203600 & -1.49155600 & 1.30904200 \\
\hline $\mathrm{H}$ & 0.12066400 & 0.22789800 & 3.84353100 \\
\hline $\mathrm{N}$ & 0.56614200 & -1.82946500 & 2.96518100 \\
\hline B & -0.11664100 & -0.94537000 & 4.07651200 \\
\hline $\mathrm{C}$ & 2.38308000 & 1.04607500 & -1.48939800 \\
\hline $\mathrm{H}$ & 3.29728900 & 1.64679800 & -1.42697600 \\
\hline $\mathrm{H}$ & 2.17249600 & 0.84938400 & -2.54695800 \\
\hline $\mathrm{C}$ & -2.24931800 & -0.59704400 & 1.31509000 \\
\hline $\mathrm{H}$ & -3.29740100 & -0.69222000 & 1.61878300 \\
\hline $\mathrm{H}$ & -1.60204300 & -0.70648100 & 2.19838500 \\
\hline $\mathrm{C}$ & 0.26131500 & -3.23788300 & 3.17418300 \\
\hline $\mathrm{H}$ & 0.56234800 & -3.85640400 & 2.31858700 \\
\hline $\mathrm{H}$ & 0.78334400 & -3.61838700 & 4.06850300 \\
\hline $\mathrm{H}$ & -0.80761500 & -3.36524400 & 3.34437400 \\
\hline $\mathrm{C}$ & 2.00663800 & -1.68061000 & 2.97731100 \\
\hline $\mathrm{H}$ & 2.46470400 & -2.32893800 & 2.21891200 \\
\hline $\mathrm{H}$ & 2.29363700 & -0.64645600 & 2.75908600 \\
\hline $\mathrm{H}$ & 2.43042400 & -1.95059400 & 3.95944200 \\
\hline
\end{tabular}

$\mathbf{T S}_{\mathbf{2}}$

$\begin{array}{lccc}\text { Fe } & -0.36301800 & 1.33302900 & -0.95260400 \\ \mathrm{P} & -2.43998900 & 0.61814700 & -0.98831600 \\ & & & \mathrm{~S} 72\end{array}$




\begin{tabular}{|c|c|c|c|}
\hline $\mathrm{P}$ & 1.73268200 & 1.69374600 & -0.41966600 \\
\hline $\mathrm{P}$ & 1.75443900 & -1.01319100 & 0.60136700 \\
\hline 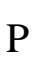 & -1.10712000 & -1.97064000 & -0.68081700 \\
\hline 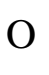 & -1.16230800 & 4.08333800 & -0.38221800 \\
\hline 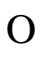 & -0.35641800 & 1.09424800 & -3.87400200 \\
\hline$C$ & -0.83907000 & 2.98468100 & -0.62848300 \\
\hline $\mathrm{C}$ & -3.50670500 & 1.67267600 & 1.33590000 \\
\hline $\mathrm{H}$ & -2.74729600 & 2.43875400 & 1.19556600 \\
\hline $\mathrm{C}$ & 3.32613300 & -1.74853300 & 0.00329500 \\
\hline C & -5.33390100 & 0.76628200 & 2.62608900 \\
\hline $\mathrm{H}$ & -6.01187600 & 0.83167700 & 3.47281400 \\
\hline $\mathrm{C}$ & 2.23765900 & 3.38008200 & 0.08616800 \\
\hline C & -0.89428300 & -3.58130800 & -1.51441500 \\
\hline $\mathrm{C}$ & 3.13148900 & 1.21558100 & -1.52586600 \\
\hline$C$ & -0.30482000 & 1.18481800 & -2.70 \\
\hline 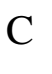 & -4.52720000 & -0.41061000 & 0.68366500 \\
\hline $\mathrm{H}$ & -4.57875700 & -1.28367500 & 0.03657200 \\
\hline $\mathrm{C}$ & -1.61630700 & -1.38495300 & 1.97604300 \\
\hline $\mathrm{H}$ & -1.08828200 & -0.45520900 & 1.77406300 \\
\hline $\mathrm{C}$ & 1.48121400 & -1.96944100 & 2.12492200 \\
\hline $\mathrm{C}$ & -1.96411700 & -4.27896800 & -2.09090200 \\
\hline $\mathrm{H}$ & -2.97049500 & -3.86807200 & -2.04590200 \\
\hline $\mathrm{B}$ & 0.38988400 & -0.79549800 & -0.75887000 \\
\hline $\mathrm{C}$ & -3.57192800 & 0.58920400 & 0.45503200 \\
\hline$C$ & -4.83135200 & 0.82168700 & -2.46579900 \\
\hline $\mathrm{H}$ & -5.15089200 & -0.04625800 & -1.89209800 \\
\hline $\mathrm{C}$ & 5.59585900 & -2.43752800 & 0.49882600 \\
\hline $\mathrm{H}$ & 6.40481900 & -2.55500200 & 1.21450300 \\
\hline $\mathrm{C}$ & 0.39170000 & -4.12535300 & -1.60338400 \\
\hline $\mathrm{H}$ & 1.23500700 & -3.57238500 & -1.19159700 \\
\hline$C$ & -3.19759000 & 2.52100500 & -2.95526700 \\
\hline $\mathrm{H}$ & -2.22364800 & 2.97017400 & -2.78773800 \\
\hline $\mathrm{C}$ & -3.56861900 & 1.38346900 & -2.23413800 \\
\hline $\mathrm{C}$ & 2.83379100 & 5.99445900 & 0.88779100 \\
\hline$\Pi$ & 3.06974400 & 7.00745200 & 1.20248400 \\
\hline $\mathrm{C}$ & 1.65398600 & 3.91850700 & 1.23841800 \\
\hline
\end{tabular}




\begin{tabular}{|c|c|c|c|}
\hline $\mathrm{H}$ & 0.94583800 & 3.32210600 & 1.81381700 \\
\hline $\mathrm{C}$ & -4.38748700 & 1.76176300 & 2.41023200 \\
\hline $\mathrm{H}$ & -4.31048600 & 2.60194900 & 3.09447200 \\
\hline $\mathrm{C}$ & -1.79026500 & -2.33168500 & 0.95989800 \\
\hline $\mathrm{C}$ & 3.39088300 & 5.47760800 & -0.27795200 \\
\hline $\mathrm{H}$ & 4.05927300 & 6.08689400 & -0.88049400 \\
\hline $\mathrm{C}$ & -3.00435800 & -3.74305200 & 2.49882400 \\
\hline $\mathrm{H}$ & -3.54186200 & -4.66525400 & 2.70360800 \\
\hline $\mathrm{C}$ & -2.82912900 & -2.79478200 & 3.50512900 \\
\hline $\mathrm{H}$ & -3.23628000 & -2.97704400 & 4.49634900 \\
\hline $\mathrm{C}$ & -5.69534200 & 1.37156000 & -3.40443100 \\
\hline $\mathrm{H}$ & -6.66997500 & 0.92097500 & -3.57212500 \\
\hline $\mathrm{C}$ & 4.37983600 & -1.90900500 & 0.91407000 \\
\hline $\mathrm{H}$ & 4.24298600 & -1.62486900 & 1.95640000 \\
\hline $\mathrm{C}$ & -1.75060900 & -5.49919200 & -2.72478200 \\
\hline $\mathrm{H}$ & -2.58763400 & -6.02867800 & -3.17105400 \\
\hline $\mathrm{C}$ & 0.60438400 & -5.34850000 & -2.23128900 \\
\hline $\mathrm{H}$ & 1.60992700 & -5.75545500 & -2.29170500 \\
\hline $\mathrm{C}$ & 5.77269900 & -2.82242400 & -0.82931800 \\
\hline $\mathrm{H}$ & 6.72349400 & -3.23622800 & -1.15401100 \\
\hline $\mathrm{C}$ & 5.29407600 & 0.45248500 & -3.14314000 \\
\hline $\mathrm{H}$ & 6.13111700 & 0.15086200 & -3.76766000 \\
\hline $\mathrm{C}$ & -2.13384800 & -1.61693000 & 3.24523500 \\
\hline $\mathrm{H}$ & -1.98320000 & -0.86237600 & 4.01219400 \\
\hline $\mathrm{C}$ & 1.05792100 & -3.30170100 & 2.02562000 \\
\hline $\mathrm{H}$ & 0.87273500 & -3.74376000 & 1.04749600 \\
\hline $\mathrm{C}$ & 2.92280200 & 0.79201600 & -2.83859400 \\
\hline $\mathrm{H}$ & 1.91435800 & 0.74249100 & -3.23218100 \\
\hline $\mathrm{C}$ & 1.96432900 & 5.21193500 & 1.64448900 \\
\hline $\mathrm{H}$ & 1.51923600 & 5.60731700 & 2.55445100 \\
\hline $\mathrm{C}$ & -5.31363000 & 2.50459200 & -4.12019500 \\
\hline $\mathrm{H}$ & -5.98915700 & 2.94034800 & -4.85130500 \\
\hline $\mathrm{C}$ & -0.46743200 & -6.03695000 & -2.79284600 \\
\hline $\mathrm{H}$ & -0.30342000 & -6.98842500 & -3.29070500 \\
\hline $\mathrm{C}$ & -5.39946300 & -0.32501000 & 1.76166000 \\
\hline $\mathrm{H}$ & -6.12528600 & -1.11620400 & $\begin{array}{l}1.93185200 \\
\text { S74 }\end{array}$ \\
\hline
\end{tabular}




\begin{tabular}{|c|c|c|c|}
\hline $\mathrm{C}$ & 3.51212500 & -2.13696700 & -1.32508300 \\
\hline $\mathrm{H}$ & 2.71036000 & -1.99644500 & -2.04500300 \\
\hline C & 1.50555600 & -2.18386200 & 4.53373000 \\
\hline $\mathrm{H}$ & 1.67371200 & -1.73802700 & 5.50972900 \\
\hline $\mathrm{C}$ & 1.05966200 & -3.49608800 & 4.42682600 \\
\hline $\mathrm{H}$ & 0.87801200 & -4.08403700 & 5.32224700 \\
\hline $\mathrm{C}$ & 0.84458300 & -4.05884600 & 3.16871900 \\
\hline $\mathrm{H}$ & 0.49333000 & -5.08295600 & 3.07846500 \\
\hline $\mathrm{C}$ & 3.09915900 & 4.17569800 & -0.67694500 \\
\hline $\mathrm{H}$ & 3.54653500 & 3.77999900 & -1.58499200 \\
\hline $\mathrm{C}$ & 4.44635000 & 1.25858200 & -1.03687200 \\
\hline $\mathrm{H}$ & 4.63447600 & 1.60237800 & -0.02102800 \\
\hline $\mathrm{C}$ & 5.51756700 & 0.87595500 & -1.83376400 \\
\hline $\mathrm{H}$ & 6.52717700 & 0.90413900 & -1.43207200 \\
\hline $\mathrm{C}$ & -2.48348400 & -3.51769400 & 1.22805900 \\
\hline $\mathrm{H}$ & -2.60471500 & -4.27063700 & 0.45257600 \\
\hline $\mathrm{C}$ & 1.72215200 & -1.42251500 & 3.38747700 \\
\hline $\mathrm{H}$ & 2.05466400 & -0.39360200 & 3.48280200 \\
\hline $\mathrm{C}$ & 3.99765700 & 0.41725100 & -3.64408900 \\
\hline $\mathrm{H}$ & 3.81396100 & 0.09072300 & -4.66441200 \\
\hline $\mathrm{C}$ & -4.06783000 & 3.07850500 & -3.89006100 \\
\hline $\mathrm{H}$ & -3.76574800 & 3.96514800 & -4.44036800 \\
\hline $\mathrm{C}$ & 4.73049600 & -2.67186200 & -1.73734300 \\
\hline $\mathrm{H}$ & 4.86410600 & -2.95792400 & -2.77739000 \\
\hline $\mathrm{H}$ & 0.93560100 & -1.08790800 & -1.79617400 \\
\hline $\mathrm{H}$ & -1.81835800 & 1.46577900 & 3.51001200 \\
\hline $\mathrm{H}$ & -0.00348000 & 0.51495900 & 3.67586700 \\
\hline $\mathrm{H}$ & -0.49721200 & 1.43070200 & 0.91626400 \\
\hline $\mathrm{H}$ & -0.52644200 & 1.51899100 & 1.97035100 \\
\hline $\mathrm{N}$ & -0.02774500 & 2.73542900 & 4.01095800 \\
\hline B & -0.61454500 & 1.52959700 & 3.41376000 \\
\hline $\mathrm{C}$ & -2.42922600 & -1.13090600 & -1.61064900 \\
\hline $\mathrm{H}$ & -3.38885800 & -1.65728100 & -1.57075700 \\
\hline $\mathrm{H}$ & -2.11106200 & -1.07296300 & -2.65821000 \\
\hline $\mathrm{C}$ & 2.17014300 & 0.68998700 & 1.08921200 \\
\hline $\mathrm{H}$ & 3.19367600 & 0.80765200 & $\begin{array}{c}1.46200000 \\
\text { S75 }\end{array}$ \\
\hline
\end{tabular}




$\begin{array}{lrrr}\mathrm{H} & 1.45775400 & 0.98809400 & 1.87208800 \\ \mathrm{C} & 1.33064500 & 2.80781700 & 4.46753000 \\ \mathrm{H} & 2.03993000 & 3.27793100 & 3.75970900 \\ \mathrm{H} & 1.40391800 & 3.38527900 & 5.40428200 \\ \mathrm{H} & 1.69893900 & 1.79551600 & 4.67107400 \\ \mathrm{C} & -0.71164700 & 3.99514800 & 3.92256700 \\ \mathrm{H} & -0.47756200 & 4.57772900 & 3.00966300 \\ \mathrm{H} & -1.79386700 & 3.82292200 & 3.91244700 \\ \mathrm{H} & -0.47452400 & 4.64074300 & 4.78294400\end{array}$

$\mathbf{T S}_{3}$

$\begin{array}{lrrr}\text { Fe } & -0.04512100 & -1.37006000 & -0.88034900 \\ \text { P } & -2.01096800 & -1.43724600 & 0.13653900 \\ \text { P } & 2.00924600 & -1.30159000 & -0.15499400 \\ \text { P } & 1.43989300 & 1.52122200 & -0.43136700 \\ \text { P } & -1.83954600 & 1.39032200 & 0.01551100 \\ \text { O } & -0.35391500 & -0.85556800 & -3.73706500 \\ \text { O } & 0.04661300 & -4.28006400 & -1.10619800 \\ \text { C } & -0.22626800 & -1.06490000 & -2.58954900 \\ \mathrm{C} & -3.36434600 & -2.32465700 & -2.16874300 \\ \mathrm{H} & -2.38243700 & -2.66045200 & -2.49728200 \\ \mathrm{C} & 1.61995300 & 3.15469200 & 0.35485800 \\ \mathrm{C} & -5.72421100 & -2.03995000 & -2.57988800 \\ \mathrm{H} & -6.58912400 & -2.16028000 & -3.22701600 \\ \mathrm{C} & 3.49915900 & -1.43429500 & -1.21977800 \\ \mathrm{C} & -1.80815000 & 2.91025700 & 1.02039800 \\ \mathrm{C} & 2.36960500 & -2.53774200 & 1.15117500 \\ \mathrm{C} & 0.01133300 & -3.12150700 & -0.97813400 \\ \mathrm{C} & -4.75424800 & -1.25800900 & -0.51507400 \\ \mathrm{H} & -4.86559400 & -0.76151000 & 0.44731400 \\ \mathrm{C} & -3.15242400 & 1.14022300 & -2.45593600 \\ \mathrm{H} & -2.30801000 & 0.52553800 & -2.75348600 \\ \mathrm{C} & 2.53278100 & 1.63410400 & -1.88512300 \\ \mathrm{C} & -1.95569900 & 2.86327300 & 2.40977900 \\ \mathrm{H} & -2.06464800 & 1.90995000 & 2.91892600 \\ \mathrm{~B} & -0.26933200 & 0.86811700 & -0.97876600 \\ & & & \mathrm{~S} 76 \\ & & & \\ & & & \end{array}$




\begin{tabular}{|c|c|c|c|}
\hline $\mathrm{C}$ & -3.49168500 & -1.70208800 & -0.92340000 \\
\hline $\mathrm{C}$ & -2.57141600 & -4.06998700 & 0.93854300 \\
\hline $\mathrm{H}$ & -2.79060900 & -4.25314700 & -0.11152700 \\
\hline $\mathrm{C}$ & 1.93095600 & 5.54768400 & 0.14730300 \\
\hline $\mathrm{H}$ & 2.12957600 & 6.41737500 & -0.47308700 \\
\hline $\mathrm{C}$ & -1.64618000 & 4.15229000 & 0.39009000 \\
\hline $\mathrm{H}$ & -1.52853200 & 4.19815200 & -0.69171400 \\
\hline $\mathrm{C}$ & -1.93117100 & -2.56844900 & 2.71370600 \\
\hline $\mathrm{H}$ & -1.63781000 & -1.58386300 & 3.06528900 \\
\hline $\mathrm{C}$ & -2.20161200 & -2.78720000 & 1.35940100 \\
\hline $\mathrm{C}$ & 5.74872700 & -1.65098100 & -2.86623800 \\
\hline $\mathrm{H}$ & 6.62286700 & -1.73473100 & -3.50650700 \\
\hline $\mathrm{C}$ & 3.35252900 & -1.68359900 & -2.58417900 \\
\hline $\mathrm{H}$ & 2.35018000 & -1.77885500 & -2.99843700 \\
\hline $\mathrm{C}$ & -4.47548900 & -2.49828500 & -2.99033100 \\
\hline $\mathrm{H}$ & -4.36144900 & -2.98145700 & -3.95715100 \\
\hline $\mathrm{C}$ & -3.17263700 & 1.73396500 & -1.18821900 \\
\hline $\mathrm{C}$ & 5.90538300 & -1.40006900 & -1.50332100 \\
\hline $\mathrm{H}$ & 6.89987800 & -1.29199700 & -1.07860000 \\
\hline $\mathrm{C}$ & -5.35639500 & 2.65931100 & -1.68762500 \\
\hline $\mathrm{H}$ & -6.21392100 & 3.25422600 & -1.38501000 \\
\hline $\mathrm{C}$ & -5.32230100 & 2.07268900 & -2.95062900 \\
\hline $\mathrm{H}$ & -6.15518500 & 2.20608400 & -3.63600800 \\
\hline $\mathrm{C}$ & -2.66400100 & -5.11266900 & 1.85376300 \\
\hline $\mathrm{H}$ & -2.95178600 & -6.10351600 & 1.51262300 \\
\hline $\mathrm{C}$ & 1.86073200 & 4.28641700 & -0.43535200 \\
\hline $\mathrm{H}$ & 2.00539500 & 4.17714200 & -1.50774400 \\
\hline $\mathrm{C}$ & -1.95866200 & 4.04134700 & 3.15415500 \\
\hline $\mathrm{H}$ & -2.08264600 & 3.98896100 & 4.23303000 \\
\hline $\mathrm{C}$ & -1.64759900 & 5.32333200 & 1.13668800 \\
\hline $\mathrm{H}$ & -1.51607000 & 6.28044400 & 0.63887800 \\
\hline $\mathrm{C}$ & 1.75120400 & 5.69019400 & 1.51972900 \\
\hline $\mathrm{H}$ & 1.80338700 & 6.67483000 & 1.97702700 \\
\hline $\mathrm{C}$ & 2.73747100 & -4.48987800 & 3.12932900 \\
\hline $\mathrm{H}$ & 2.88076800 & -5.24699200 & 3.89528200 \\
\hline $\mathrm{C}$ & -4.22060800 & 1.31075800 & $\begin{array}{c}-3.33006900 \\
\text { S77 }\end{array}$ \\
\hline
\end{tabular}




\begin{tabular}{|c|c|c|c|}
\hline $\mathrm{H}$ & -4.19210600 & 0.83651000 & -4.30749900 \\
\hline $\mathrm{C}$ & 2.05659200 & 1.46980700 & -3.18808100 \\
\hline $\mathrm{H}$ & 1.00910900 & 1.25270000 & -3.36288000 \\
\hline $\mathrm{C}$ & 3.27480400 & -3.58644000 & 0.95438900 \\
\hline $\mathrm{H}$ & 3.83922000 & -3.65737400 & 0.02900700 \\
\hline $\mathrm{C}$ & 4.47375700 & -1.79198700 & -3.40502700 \\
\hline $\mathrm{H}$ & 4.34747000 & -1.97901800 & -4.46804700 \\
\hline $\mathrm{C}$ & -2.39395200 & -4.88701500 & 3.20201400 \\
\hline $\mathrm{H}$ & -2.47113400 & -5.70190000 & 3.91698000 \\
\hline $\mathrm{C}$ & -1.80965600 & 5.27043900 & 2.52122000 \\
\hline $\mathrm{H}$ & -1.81309300 & 6.18847000 & 3.10316700 \\
\hline $\mathrm{C}$ & -5.86189200 & -1.42037200 & -1.33871800 \\
\hline $\mathrm{H}$ & -6.83331300 & -1.05475700 & -1.01577500 \\
\hline $\mathrm{C}$ & 1.43108100 & 3.30301700 & 1.73548700 \\
\hline $\mathrm{H}$ & 1.22061500 & 2.43636400 & 2.36505300 \\
\hline $\mathrm{C}$ & 4.76006900 & 1.99752300 & -2.76694900 \\
\hline $\mathrm{H}$ & 5.81532200 & 2.19231400 & -2.59673100 \\
\hline $\mathrm{C}$ & 4.27646600 & 1.83197000 & -4.06387200 \\
\hline $\mathrm{H}$ & 4.95582200 & 1.90011500 & -4.90926300 \\
\hline $\mathrm{C}$ & 2.92677400 & 1.57077200 & -4.27077100 \\
\hline $\mathrm{H}$ & 2.54216800 & 1.43472400 & -5.27755300 \\
\hline $\mathrm{C}$ & 4.78732700 & -1.29307800 & -0.68515700 \\
\hline $\mathrm{H}$ & 4.91352600 & -1.11786700 & 0.38263100 \\
\hline $\mathrm{C}$ & 1.63575800 & -2.49435400 & 2.34216900 \\
\hline $\mathrm{H}$ & 0.89817300 & -1.71027500 & 2.50593400 \\
\hline $\mathrm{C}$ & 1.82787000 & -3.45391500 & 3.32931400 \\
\hline $\mathrm{H}$ & 1.25365200 & -3.38682800 & 4.25014400 \\
\hline $\mathrm{C}$ & -4.29083800 & 2.48879000 & -0.80978400 \\
\hline $\mathrm{H}$ & -4.32178800 & 2.95159000 & 0.17492400 \\
\hline $\mathrm{C}$ & 3.89414300 & 1.90314000 & -1.68538400 \\
\hline $\mathrm{H}$ & 4.28099600 & 2.03861800 & -0.67669400 \\
\hline $\mathrm{C}$ & 3.45244600 & -4.55705800 & 1.93787200 \\
\hline $\mathrm{H}$ & 4.15382100 & -5.36927000 & 1.76691800 \\
\hline $\mathrm{C}$ & -2.03056700 & -3.61320400 & 3.62825500 \\
\hline $\mathrm{H}$ & -1.81952900 & -3.42170300 & 4.67764500 \\
\hline $\mathrm{C}$ & 1.49658500 & 4.57025100 & $\begin{array}{c}2.30739800 \\
\text { S78 }\end{array}$ \\
\hline
\end{tabular}




$\begin{array}{lrrr}\mathrm{H} & 1.33596000 & 4.68275600 & 3.37573700 \\ \mathrm{H} & -0.42525500 & 1.39750200 & -2.04879200 \\ \mathrm{H} & 0.08163100 & -1.15017100 & 0.64048800 \\ \mathrm{H} & -1.06557100 & 0.15136600 & 2.06837400 \\ \mathrm{H} & -1.06828500 & 0.76417600 & 4.57243100 \\ \mathrm{H} & 0.05766700 & -0.94550000 & 4.31883200 \\ \mathrm{H} & -0.35822200 & 0.24614300 & 2.69562700 \\ \mathrm{~N} & 1.17477600 & 1.01325400 & 4.45760200 \\ \mathrm{~B} & -0.03693400 & 0.25232800 & 4.20894800 \\ \mathrm{C} & -2.23459500 & 0.05997700 & 1.09303900 \\ \mathrm{H} & -3.16916700 & 0.15215400 & 1.65515100 \\ \mathrm{C} & 2.48433700 & 0.41845200 & 4.47715300 \\ \mathrm{H} & 2.44350200 & -0.60344900 & 4.08969900 \\ \mathrm{H} & 3.20391500 & 0.98947000 & 3.86243600 \\ \mathrm{H} & 2.91016700 & 0.37719400 & 5.49498600 \\ \mathrm{C} & 1.16138200 & 2.33935200 & 5.01242600 \\ \mathrm{H} & 1.95175400 & 2.96902600 & 4.57155600 \\ \mathrm{H} & 0.19656000 & 2.82155000 & 4.82553400 \\ \mathrm{H} & 1.33773600 & 2.34242600 & 6.10299700 \\ \mathrm{C} & 2.25891100 & 0.33300600 & 0.68323800 \\ \mathrm{H} & 3.30139800 & 0.59259500 & 0.89606900 \\ \mathrm{H} & 1.69393600 & 0.31337900 & 1.62647100\end{array}$

\section{$\mathrm{TS}_{4}$}

$\begin{array}{lrrc}\mathrm{Fe} & 0.35099400 & -1.24167400 & -0.79955600 \\ \mathrm{P} & -1.75412100 & -1.53620600 & -0.26981800 \\ \mathrm{P} & 2.23809300 & -1.06611300 & 0.29858600 \\ \mathrm{P} & 1.56789900 & 1.74283300 & 0.11580700 \\ \mathrm{P} & -1.71176500 & 1.36068900 & -0.34808600 \\ \mathrm{O} & 0.69860100 & -0.70477100 & -3.64378500 \\ \mathrm{O} & 0.71344400 & -4.12111400 & -1.09180200 \\ \mathrm{C} & 0.55726100 & -0.91940700 & -2.49424400 \\ \mathrm{C} & -2.43399000 & -2.58258600 & -2.75507800 \\ \mathrm{H} & -1.38981900 & -2.88511100 & -2.76799500 \\ \mathrm{C} & 1.32454600 & 3.25370400 & 1.10387400 \\ \mathrm{C} & -4.58202700 & -2.44973500 & -3.84559500 \\ & & & \mathrm{~S} 79\end{array}$




\begin{tabular}{|c|c|c|c|}
\hline $\mathrm{H}$ & -5.21944700 & -2.66028100 & -4.70018700 \\
\hline $\mathrm{C}$ & 3.79054600 & -1.09775100 & -0.69677500 \\
\hline $\mathrm{C}$ & -2.12569300 & 2.86000700 & 0.59469000 \\
\hline $\mathrm{C}$ & 2.63367100 & -2.33595700 & 1.55936500 \\
\hline $\mathrm{C}$ & 0.56654500 & -2.96804900 & -0.93878800 \\
\hline $\mathrm{C}$ & -4.26803100 & -1.47777200 & -1.65994100 \\
\hline $\mathrm{H}$ & -4.68313000 & -0.92057600 & -0.82307300 \\
\hline $\mathrm{C}$ & -2.28501000 & 1.03344000 & -3.08562800 \\
\hline $\mathrm{H}$ & -1.30235400 & 0.58196200 & -3.17070500 \\
\hline $\mathrm{C}$ & 2.93439900 & 2.13933900 & -1.02000700 \\
\hline $\mathrm{C}$ & -2.75116400 & 2.80720400 & 1.84330700 \\
\hline $\mathrm{H}$ & -2.99370600 & 1.84587200 & 2.28908900 \\
\hline B & 0.10210600 & 0.97247500 & -0.85673800 \\
\hline $\mathrm{C}$ & -2.92841700 & -1.88566500 & -1.64563600 \\
\hline $\mathrm{C}$ & -3.48682300 & -3.27671100 & 1.16327700 \\
\hline $\mathrm{H}$ & -4.29313100 & -2.88363900 & 0.54733800 \\
\hline $\mathrm{C}$ & 1.36980300 & 5.66326300 & 1.33667500 \\
\hline $\mathrm{H}$ & 1.62574500 & 6.63955500 & 0.93347500 \\
\hline $\mathrm{C}$ & -1.79274100 & 4.10504600 & 0.04267500 \\
\hline $\mathrm{H}$ & -1.30262200 & 4.15122900 & -0.92906200 \\
\hline $\mathrm{C}$ & -1.14859600 & -3.37802300 & 1.74516400 \\
\hline $\mathrm{H}$ & -0.12507400 & -3.03991000 & 1.59561700 \\
\hline $\mathrm{C}$ & -2.17144500 & -2.84150900 & 0.95613100 \\
\hline $\mathrm{C}$ & 6.10874600 & -1.24440500 & -2.26620600 \\
\hline $\mathrm{H}$ & 7.00647700 & -1.29216200 & -2.87687600 \\
\hline $\mathrm{C}$ & 3.76396200 & -1.71208300 & -1.95232000 \\
\hline $\mathrm{H}$ & 2.82521300 & -2.12002400 & -2.31883400 \\
\hline $\mathrm{C}$ & -3.25393200 & -2.86784800 & -3.84203600 \\
\hline $\mathrm{H}$ & -2.84895600 & -3.40948300 & -4.69267300 \\
\hline $\mathrm{C}$ & -2.72189300 & 1.53579000 & -1.85649900 \\
\hline $\mathrm{C}$ & 6.14833600 & -0.63328900 & -1.01476000 \\
\hline $\mathrm{H}$ & 7.07594000 & -0.20141000 & -0.64734500 \\
\hline $\mathrm{C}$ & -4.83148300 & 2.14993600 & -2.87315100 \\
\hline $\mathrm{H}$ & -5.82153400 & 2.58944400 & -2.78775500 \\
\hline $\mathrm{C}$ & -4.39130800 & 1.64353700 & -4.09441500 \\
\hline $\mathrm{H}$ & -5.04170100 & 1.68099400 & $\begin{array}{c}-4.96419200 \\
\text { S80 }\end{array}$ \\
\hline
\end{tabular}




\begin{tabular}{|c|c|c|c|}
\hline $\mathrm{C}$ & -3.77123200 & -4.22011000 & 2.14515300 \\
\hline $\mathrm{H}$ & -4.79551400 & -4.55201200 & 2.29439900 \\
\hline C & 1.63489000 & 4.51947200 & 0.59068300 \\
\hline $\mathrm{H}$ & 2.09615200 & 4.60788900 & -0.38968000 \\
\hline $\mathrm{C}$ & -3.04333900 & 3.98565000 & 2.52712000 \\
\hline $\mathrm{H}$ & -3.52676400 & 3.93381300 & 3.49954000 \\
\hline $\mathrm{C}$ & -2.08629500 & 5.27518100 & 0.72824500 \\
\hline $\mathrm{H}$ & -1.81689800 & 6.23545300 & 0.29656500 \\
\hline $\mathrm{C}$ & 0.77631300 & 5.55738100 & 2.59133800 \\
\hline $\mathrm{H}$ & 0.56299000 & 6.45123000 & 3.17145200 \\
\hline $\mathrm{C}$ & 2.97216900 & -4.45606100 & 3.36311900 \\
\hline $\mathrm{H}$ & 3.09632000 & -5.27896600 & 4.06215100 \\
\hline $\mathrm{C}$ & -3.12032000 & 1.08647000 & -4.19757800 \\
\hline $\mathrm{H}$ & -2.77433600 & 0.67859300 & -5.14309000 \\
\hline $\mathrm{C}$ & 2.93296700 & 1.70242100 & -2.34678100 \\
\hline $\mathrm{H}$ & 2.07700300 & 1.16545100 & -2.74301600 \\
\hline $\mathrm{C}$ & 3.06444000 & -3.59407400 & 1.11201300 \\
\hline $\mathrm{H}$ & 3.26059400 & -3.75481900 & 0.05401900 \\
\hline $\mathrm{C}$ & 4.91677100 & -1.79356900 & -2.72884000 \\
\hline $\mathrm{H}$ & 4.87820900 & -2.27805200 & -3.70091000 \\
\hline $\mathrm{C}$ & -2.74546500 & -4.73923100 & 2.93371600 \\
\hline $\mathrm{H}$ & -2.96984200 & -5.47373900 & 3.70276000 \\
\hline $\mathrm{C}$ & -2.71420800 & 5.21740200 & 1.97246700 \\
\hline $\mathrm{H}$ & -2.94024700 & 6.13478700 & 2.50962600 \\
\hline $\mathrm{C}$ & -5.08688200 & -1.75292800 & -2.75142200 \\
\hline $\mathrm{H}$ & -6.11839900 & -1.40967500 & -2.74944700 \\
\hline $\mathrm{C}$ & 0.71246000 & 3.15526600 & 2.36110000 \\
\hline $\mathrm{H}$ & 0.43976100 & 2.17829900 & 2.76340000 \\
\hline $\mathrm{C}$ & 5.17299200 & 3.01504300 & -1.32373700 \\
\hline $\mathrm{H}$ & 6.04494900 & 3.52283800 & -0.92042000 \\
\hline $\mathrm{C}$ & 5.16290200 & 2.57973100 & -2.64770400 \\
\hline $\mathrm{H}$ & 6.03156500 & 2.74386500 & -3.27972800 \\
\hline $\mathrm{C}$ & 4.04695100 & 1.92218800 & -3.15376500 \\
\hline $\mathrm{H}$ & 4.04100600 & 1.56219900 & -4.17861600 \\
\hline $\mathrm{C}$ & 5.00077300 & -0.57210200 & -0.23058000 \\
\hline $\mathrm{H}$ & 5.05369600 & -0.10657800 & $\begin{array}{c}0.75205100 \\
\quad S 81\end{array}$ \\
\hline
\end{tabular}




\begin{tabular}{|c|c|c|c|}
\hline $\mathrm{C}$ & 2.38923600 & -2.15358900 & 2.92317200 \\
\hline $\mathrm{H}$ & 2.04213700 & -1.19847900 & 3.30478700 \\
\hline $\mathrm{C}$ & 2.55414000 & -3.20944200 & 3.81664100 \\
\hline $\mathrm{H}$ & 2.34242000 & -3.04907900 & 4.87065800 \\
\hline $\mathrm{C}$ & -4.00269500 & 2.09517800 & -1.75813200 \\
\hline $\mathrm{H}$ & -4.34941600 & 2.49305100 & -0.80608900 \\
\hline $\mathrm{C}$ & 4.06939800 & 2.78772200 & -0.51122800 \\
\hline $\mathrm{H}$ & 4.08555900 & 3.12116100 & 0.52504100 \\
\hline $\mathrm{C}$ & 3.23159300 & -4.64386100 & 2.00630600 \\
\hline $\mathrm{H}$ & 3.56205000 & -5.61235500 & 1.64100100 \\
\hline $\mathrm{C}$ & -1.43515500 & -4.31765300 & 2.73110800 \\
\hline $\mathrm{H}$ & -0.62659100 & -4.70792300 & 3.34374000 \\
\hline $\mathrm{C}$ & 0.44245800 & 4.30289200 & 3.09730100 \\
\hline $\mathrm{H}$ & -0.03614200 & 4.21489600 & 4.06939300 \\
\hline $\mathrm{H}$ & 0.18295600 & 1.55137700 & -1.91524500 \\
\hline $\mathrm{H}$ & 0.00130900 & -0.85749700 & 1.13560900 \\
\hline $\mathrm{H}$ & -2.10581700 & -0.01052700 & 1.58066100 \\
\hline $\mathrm{H}$ & 0.47764000 & 0.29750900 & 3.83558700 \\
\hline $\mathrm{H}$ & -0.25143100 & -1.62768200 & 3.82770900 \\
\hline $\mathrm{H}$ & -0.19524300 & -0.61051500 & 2.09280100 \\
\hline $\mathrm{N}$ & -1.77237600 & 0.08240200 & 3.82581600 \\
\hline B & -0.42660500 & -0.46566300 & 3.55524900 \\
\hline $\mathrm{C}$ & 2.32004100 & 0.52650600 & 1.23602700 \\
\hline $\mathrm{H}$ & 3.31216600 & 0.83823800 & 1.57701800 \\
\hline $\mathrm{H}$ & 1.66503700 & 0.41881600 & 2.10813200 \\
\hline $\mathrm{C}$ & -2.46389900 & -0.02761200 & 0.54143900 \\
\hline $\mathrm{H}$ & -3.55706000 & 0.03538300 & 0.55791100 \\
\hline $\mathrm{C}$ & -1.90603400 & 1.25609200 & 4.65156000 \\
\hline $\mathrm{H}$ & -1.13842400 & 1.99455100 & 4.39844600 \\
\hline $\mathrm{H}$ & -2.89012300 & 1.73478000 & 4.51941500 \\
\hline $\mathrm{H}$ & -1.81109900 & 1.03310000 & 5.73077000 \\
\hline $\mathrm{C}$ & -2.86109000 & -0.84566500 & 4.03406100 \\
\hline $\mathrm{H}$ & -3.83069300 & -0.39622900 & 3.76042100 \\
\hline $\mathrm{H}$ & -2.71622800 & -1.73824400 & 3.41996700 \\
\hline $\mathrm{H}$ & -2.94467500 & -1.17473800 & 5.08567900 \\
\hline
\end{tabular}


TS iso1

\begin{tabular}{|c|c|c|c|}
\hline e & -0.18354400 & -1.56611800 & -0.47441700 \\
\hline $\mathrm{P}$ & -2.20766100 & -1.18381800 & 0.46437200 \\
\hline $\mathrm{P}$ & 2.01788300 & -1.58023400 & -1.00120600 \\
\hline $\mathrm{P}$ & 1.86839400 & 1.41328000 & -0.66926300 \\
\hline $\mathrm{P}$ & -1.40186300 & 1.71484600 & 0.31786500 \\
\hline $\mathrm{O}$ & -0.85172800 & -1.17319700 & -3.31498900 \\
\hline $\mathrm{O}$ & -0.37945200 & -4.47344300 & -0.53751900 \\
\hline $\mathrm{C}$ & -0.61417000 & -1.32593400 & -2.18604300 \\
\hline$C$ & -3.68108000 & -2.01820300 & -1.74600800 \\
\hline $\mathrm{H}$ & -2.87400800 & -2.73653500 & -1.87412500 \\
\hline $\mathrm{C}$ & 2.98234700 & 1.67714400 & 0.71728800 \\
\hline $\mathrm{C}$ & -5.79415500 & -1.10722700 & -2.47035100 \\
\hline $\mathrm{H}$ & -6.62911300 & -1.10528300 & -3.16479000 \\
\hline $\mathrm{C}$ & 2.45903100 & -2.76629500 & -2.31198400 \\
\hline $\mathrm{C}$ & -1.03454900 & 3.16113100 & 1.33649700 \\
\hline $\mathrm{C}$ & 3.30845100 & -1.80713400 & 0.26078800 \\
\hline $\mathrm{C}$ & -0.31907400 & -3.31539000 & -0.51960300 \\
\hline $\mathrm{C}$ & -4.68906200 & -0.20101300 & -0.52930900 \\
\hline $\mathrm{H}$ & -4.68340500 & 0.52635700 & 0.27770900 \\
\hline $\mathrm{C}$ & -2.61917600 & 1.76597800 & -2.22849700 \\
\hline $\mathrm{H}$ & -1.96022200 & 0.95060200 & -2.50998800 \\
\hline $\mathrm{C}$ & 1.90493300 & 2.92178900 & -1.68082200 \\
\hline $\mathrm{C}$ & -1.32924000 & 3.22586500 & 2.70038900 \\
\hline 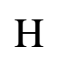 & -1.83300100 & 2.40077300 & 3.19437100 \\
\hline D & 0.08052500 & 0.74505900 & -0.44234400 \\
\hline $\mathrm{C}$ & -3.62839600 & -1.10131900 & -0.68867100 \\
\hline $\mathrm{C}$ & -4.12948500 & -2.18359000 & 2.23697600 \\
\hline $\mathrm{H}$ & -4.77744700 & -1.40257100 & 1.84434200 \\
\hline$C$ & 5.20463300 & 1.74934300 & 1.65860800 \\
\hline 1 & 6.27069100 & 1.56379400 & 1.56632900 \\
\hline $\mathrm{C}$ & -0.38165900 & 4.23535600 & 0.71181900 \\
\hline $\mathbf{H}$ & -0.18057800 & 4.20460600 & -0.35768200 \\
\hline & -2.01373400 & -3.34502200 & 2.25484000 \\
\hline & -0.98621900 & -3.43556500 & 1.91869000 \\
\hline & -2.82104800 & -2.32330100 & 1.75313200 \\
\hline
\end{tabular}




\begin{tabular}{|c|c|c|c|}
\hline $\mathrm{C}$ & 2.94278900 & -4.71306000 & -4.26216500 \\
\hline $\mathrm{H}$ & 3.13318400 & -5.46711400 & -5.01964500 \\
\hline $\mathrm{C}$ & 2.85005600 & -4.06069400 & -1.94180000 \\
\hline $\mathrm{H}$ & 2.97087000 & -4.31132000 & -0.89006800 \\
\hline $\mathrm{C}$ & -4.75511300 & -2.02084800 & -2.63009100 \\
\hline $\mathrm{H}$ & -4.77790200 & -2.73740300 & -3.44568700 \\
\hline $\mathrm{C}$ & -2.55324000 & 2.32722900 & -0.94732500 \\
\hline $\mathrm{C}$ & 2.54611700 & -3.43282200 & -4.63774500 \\
\hline $\mathrm{H}$ & 2.42776900 & -3.18367700 & -5.68802000 \\
\hline $\mathrm{C}$ & -4.38038500 & 3.80956800 & -1.52164200 \\
\hline $\mathrm{H}$ & -5.06180600 & 4.60845700 & -1.24495600 \\
\hline $\mathrm{C}$ & -4.43426100 & 3.25127300 & -2.79676900 \\
\hline $\mathrm{H}$ & -5.16174100 & 3.61329500 & -3.51721300 \\
\hline $\mathrm{C}$ & -4.61783800 & -3.05506600 & 3.20400300 \\
\hline $\mathrm{H}$ & -5.63248900 & -2.93682100 & 3.57249100 \\
\hline $\mathrm{C}$ & 4.36211200 & 1.45979100 & 0.59323600 \\
\hline $\mathrm{H}$ & 4.78047900 & 1.05764500 & -0.32634100 \\
\hline $\mathrm{C}$ & -0.94655500 & 4.34334300 & 3.43885900 \\
\hline $\mathrm{H}$ & -1.17138300 & 4.38526200 & 4.49990400 \\
\hline $\mathrm{C}$ & 0.00119700 & 5.34383000 & 1.45537500 \\
\hline $\mathrm{H}$ & 0.51272700 & 6.16810900 & 0.96674500 \\
\hline $\mathrm{C}$ & 4.68057900 & 2.26343500 & 2.84494100 \\
\hline $\mathrm{H}$ & 5.34267100 & 2.48055700 & 3.67803600 \\
\hline $\mathrm{C}$ & 5.29938700 & -1.92654900 & 2.21198300 \\
\hline $\mathrm{H}$ & 6.07652400 & -1.97725000 & 2.96953600 \\
\hline $\mathrm{C}$ & -3.55531200 & 2.23017800 & -3.14705900 \\
\hline $\mathrm{H}$ & -3.60042300 & 1.78294800 & -4.13566100 \\
\hline $\mathrm{C}$ & 1.11915400 & 2.99178900 & -2.84063400 \\
\hline $\mathrm{H}$ & 0.53287100 & 2.13225300 & -3.16112300 \\
\hline $\mathrm{C}$ & 4.64004700 & -2.05444800 & -0.10857600 \\
\hline $\mathrm{H}$ & 4.89971600 & -2.21064600 & -1.15418600 \\
\hline $\mathrm{C}$ & 3.09154200 & -5.02562200 & -2.91327600 \\
\hline $\mathrm{H}$ & 3.39709200 & -6.02359200 & -2.61391000 \\
\hline $\mathrm{C}$ & -3.81111900 & -4.08292000 & 3.68736300 \\
\hline $\mathrm{H}$ & -4.19726500 & -4.77164500 & 4.43279700 \\
\hline $\mathrm{C}$ & -0.27450200 & 5.39420100 & $\begin{array}{c}2.82193600 \\
\text { S84 }\end{array}$ \\
\hline
\end{tabular}




\begin{tabular}{|c|c|c|c|}
\hline $\mathrm{H}$ & 0.02741000 & 6.25953100 & 3.40414300 \\
\hline $\mathrm{C}$ & -5.76107600 & -0.20067700 & -1.41486000 \\
\hline $\mathrm{H}$ & -6.56561200 & 0.51726100 & -1.28275100 \\
\hline $\mathrm{C}$ & 2.46303900 & 2.20226100 & 1.90496700 \\
\hline $\mathrm{H}$ & 1.39358200 & 2.35423300 & 2.01770600 \\
\hline $\mathrm{C}$ & 2.59563200 & 5.21219600 & -2.03089200 \\
\hline $\mathrm{H}$ & 3.17488700 & 6.07506200 & -1.71649400 \\
\hline $\mathrm{C}$ & 1.80745000 & 5.27862500 & -3.17607600 \\
\hline $\mathrm{H}$ & 1.76774300 & 6.19572500 & -3.75598100 \\
\hline $\mathrm{C}$ & 1.07239700 & 4.16596200 & -3.58247600 \\
\hline $\mathrm{H}$ & 0.45999500 & 4.21204800 & -4.47768300 \\
\hline $\mathrm{C}$ & 2.29942600 & -2.46470700 & -3.66906700 \\
\hline $\mathrm{H}$ & 1.97824400 & -1.47539800 & -3.98476800 \\
\hline $\mathrm{C}$ & 2.99001200 & -1.61823000 & 1.60365300 \\
\hline $\mathrm{H}$ & 1.96539800 & -1.40080900 & 1.88281100 \\
\hline $\mathrm{C}$ & 3.98257800 & -1.66892900 & 2.57850800 \\
\hline $\mathrm{H}$ & 3.71250300 & -1.49732600 & 3.61759400 \\
\hline $\mathrm{C}$ & -3.44408000 & 3.35293400 & -0.60069200 \\
\hline $\mathrm{H}$ & -3.39716400 & 3.80326500 & 0.38837900 \\
\hline $\mathrm{C}$ & 2.64689300 & 4.03954900 & -1.28154900 \\
\hline $\mathrm{H}$ & 3.25459700 & 3.99704500 & -0.38116300 \\
\hline $\mathrm{C}$ & 5.62677500 & -2.11978200 & 0.86862700 \\
\hline $\mathrm{H}$ & 6.65426200 & -2.32613200 & 0.58285000 \\
\hline $\mathrm{C}$ & -2.51057700 & -4.22476600 & 3.21300600 \\
\hline $\mathrm{H}$ & -1.87349500 & -5.01933800 & 3.59116000 \\
\hline $\mathrm{C}$ & 3.31369300 & 2.49216800 & 2.96597800 \\
\hline $\mathrm{H}$ & 2.89405000 & 2.87415100 & 3.89173300 \\
\hline $\mathrm{H}$ & -0.14857300 & 0.79728300 & -1.62381600 \\
\hline $\mathrm{H}$ & 0.31969400 & -0.06518400 & 0.80947300 \\
\hline $\mathrm{H}$ & 1.71024700 & 0.01605100 & 3.50609000 \\
\hline $\mathrm{H}$ & 0.04833300 & 1.20258800 & 3.59380800 \\
\hline $\mathrm{H}$ & 0.16104200 & -1.83374700 & 1.00184600 \\
\hline $\mathrm{H}$ & 0.49537800 & 0.16738200 & 1.95293800 \\
\hline $\mathrm{N}$ & -0.29165800 & -1.01991400 & 3.81904200 \\
\hline B & 0.51740700 & 0.11724900 & 3.31788700 \\
\hline $\mathrm{C}$ & -2.23765500 & 0.45443300 & $\begin{array}{c}1.33165000 \\
\text { S85 }\end{array}$ \\
\hline
\end{tabular}




$\begin{array}{lrrr}\mathrm{H} & -3.23584200 & 0.75528900 & 1.66407800 \\ \mathrm{H} & -1.60886300 & 0.30570700 & 2.21788600 \\ \mathrm{C} & 2.50765400 & 0.06117500 & -1.72155500 \\ \mathrm{H} & 3.58664800 & 0.12308600 & -1.89647300 \\ \mathrm{H} & 1.99964400 & 0.18495900 & -2.68490300 \\ \mathrm{C} & -1.47316300 & -0.79277900 & 4.61740600 \\ \mathrm{H} & -1.96364000 & 0.14201200 & 4.32426700 \\ \mathrm{H} & -2.20364500 & -1.61031100 & 4.50894000 \\ \mathrm{H} & -1.24389000 & -0.71005800 & 5.69515800 \\ \mathrm{C} & 0.38393900 & -2.23000200 & 4.22698700 \\ \mathrm{H} & 0.87161900 & -2.12603100 & 5.21297800 \\ \mathrm{H} & -0.31955600 & -3.07008200 & 4.30580800 \\ \mathrm{H} & 1.15669700 & -2.50985900 & 3.50449400\end{array}$

TS $_{\text {iso2 }}$

$\begin{array}{lrrr}\mathrm{Fe} & -0.00076800 & -1.43031900 & 0.28965800 \\ \mathrm{P} & 2.08543100 & -1.31189000 & -0.66177200 \\ \mathrm{P} & -2.11792100 & -1.32227300 & 1.12933400 \\ \mathrm{P} & -1.66288400 & 1.59614000 & 0.68466500 \\ \mathrm{P} & 1.54220200 & 1.55834000 & -0.16406200 \\ \mathrm{O} & 0.99768900 & -1.43044600 & 3.00038700 \\ \mathrm{O} & -0.44481600 & -4.31997300 & -0.15957300 \\ \mathrm{C} & 0.60406900 & -1.44846500 & 1.90119800 \\ \mathrm{C} & 3.54304000 & -2.36642900 & 1.47419200 \\ \mathrm{H} & 2.65850400 & -2.97061300 & 1.65865900 \\ \mathrm{C} & -2.61048300 & 1.90830600 & -0.83189800 \\ \mathrm{C} & 5.82044100 & -1.79873500 & 2.02787500 \\ \mathrm{H} & 6.70122900 & -1.93896400 & 2.64762300 \\ \mathrm{C} & -2.33886300 & -2.30939400 & 2.65240800 \\ \mathrm{C} & 1.25610900 & 3.10764000 & -1.06414900 \\ \mathrm{C} & -3.53251000 & -1.85181200 & 0.11663600 \\ \mathrm{C} & -0.23903500 & -3.18622000 & -0.03161300 \\ \mathrm{C} & 4.71516300 & -0.69794200 & 0.18900400 \\ \mathrm{H} & 4.75721600 & 0.02160500 & -0.62537000 \\ \mathrm{C} & 3.00670400 & 1.39187400 & 2.23459600 \\ \mathrm{H} & 2.22033900 & 0.74277400 & 2.60893600 \\ & & & \mathrm{~S} 86 \\ & & & \\ & & & \end{array}$




\begin{tabular}{|c|c|c|c|}
\hline $\mathrm{C}$ & -1.69742500 & 3.17843400 & 1.60026300 \\
\hline $\mathrm{C}$ & 1.25830600 & 3.17999700 & -2.46118800 \\
\hline $\mathrm{H}$ & 1.50294100 & 2.30647400 & -3.05921400 \\
\hline B & 0.07434200 & 0.74362400 & 0.76515500 \\
\hline $\mathrm{C}$ & 3.54263800 & -1.42124600 & 0.44267400 \\
\hline $\mathrm{C}$ & 3.24954000 & -1.91537600 & -3.16453600 \\
\hline $\mathrm{H}$ & 3.37402500 & -0.84620300 & -3.31790500 \\
\hline $\mathrm{C}$ & -4.54899500 & 1.57652100 & -2.24342900 \\
\hline $\mathrm{H}$ & -5.48875200 & 1.06544500 & -2.43258200 \\
\hline $\mathrm{C}$ & 0.94950600 & 4.25289600 & -0.31306800 \\
\hline $\mathrm{H}$ & 0.95886300 & 4.20899400 & 0.77460200 \\
\hline $\mathrm{C}$ & 2.50778200 & -3.78842100 & -1.83608300 \\
\hline $\mathrm{H}$ & 2.04830700 & -4.18698400 & -0.93527300 \\
\hline $\mathrm{C}$ & 2.64288700 & -2.40741800 & -2.00621400 \\
\hline $\mathrm{C}$ & -2.55209000 & -3.92000300 & 4.93142000 \\
\hline $\mathrm{H}$ & -2.63887100 & -4.54293800 & 5.81649800 \\
\hline $\mathrm{C}$ & -2.84665600 & -3.61203500 & 2.55622900 \\
\hline $\mathrm{H}$ & -3.16608000 & -4.00147900 & 1.59280800 \\
\hline $\mathrm{C}$ & 4.67329500 & -2.54929100 & 2.26555800 \\
\hline $\mathrm{H}$ & 4.65484500 & -3.28274600 & 3.06609400 \\
\hline $\mathrm{C}$ & 2.92577200 & 1.93648400 & 0.94809000 \\
\hline $\mathrm{C}$ & -2.03387000 & -2.63206700 & 5.03364100 \\
\hline $\mathrm{H}$ & -1.71087600 & -2.24700800 & 5.99607100 \\
\hline $\mathrm{C}$ & 5.07018300 & 3.00994200 & 1.27545000 \\
\hline $\mathrm{H}$ & 5.86910300 & 3.64445800 & 0.90350200 \\
\hline $\mathrm{C}$ & 5.14580800 & 2.46359200 & 2.55554600 \\
\hline $\mathrm{H}$ & 6.00866200 & 2.66969500 & 3.18200200 \\
\hline $\mathrm{C}$ & 3.69339800 & -2.79289000 & -4.14924900 \\
\hline $\mathrm{H}$ & 4.15470200 & -2.39962800 & -5.05046000 \\
\hline $\mathrm{C}$ & -3.82555800 & 1.26761900 & -1.09386100 \\
\hline $\mathrm{H}$ & -4.22409800 & 0.52446200 & -0.41219600 \\
\hline $\mathrm{C}$ & 0.93680400 & 4.37615200 & -3.09846000 \\
\hline $\mathrm{H}$ & 0.94086700 & 4.42396900 & -4.18352500 \\
\hline $\mathrm{C}$ & 0.62735000 & 5.44274000 & -0.95451600 \\
\hline $\mathrm{H}$ & 0.38669800 & 6.32264100 & -0.36534300 \\
\hline $\mathrm{C}$ & -4.07219600 & 2.52820600 & -3.13849600 \\
\hline
\end{tabular}




\begin{tabular}{|c|c|c|c|}
\hline $\mathrm{H}$ & -4.64002000 & 2.76821500 & -4.03248900 \\
\hline $\mathrm{C}$ & -5.70679100 & -2.60033000 & -1.46020900 \\
\hline $\mathrm{H}$ & -6.55489100 & -2.89235200 & -2.07263100 \\
\hline $\mathrm{C}$ & 4.11652600 & 1.65694300 & 3.03159900 \\
\hline $\mathrm{H}$ & 4.17650500 & 1.22342000 & 4.02536100 \\
\hline $\mathrm{C}$ & -0.90302600 & 3.31898500 & 2.74963600 \\
\hline $\mathrm{H}$ & -0.29306100 & 2.48958800 & 3.09902700 \\
\hline $\mathrm{C}$ & -4.83244600 & -1.80953200 & 0.64546500 \\
\hline $\mathrm{H}$ & -4.99522300 & -1.50347500 & 1.67803000 \\
\hline $\mathrm{C}$ & -2.95304300 & -4.40911600 & 3.69106700 \\
\hline $\mathrm{H}$ & -3.35350800 & -5.41454300 & 3.60330500 \\
\hline $\mathrm{C}$ & 3.53999800 & -4.16537600 & -3.98240200 \\
\hline $\mathrm{H}$ & 3.88024400 & -4.84852200 & -4.75467900 \\
\hline $\mathrm{C}$ & 0.61324900 & 5.50379200 & -2.34807300 \\
\hline $\mathrm{H}$ & 0.36035800 & 6.43402900 & -2.84827200 \\
\hline $\mathrm{C}$ & 5.84135000 & -0.87781000 & 0.98269200 \\
\hline $\mathrm{H}$ & 6.73675100 & -0.29600700 & 0.78254700 \\
\hline $\mathrm{C}$ & -2.13971700 & 2.87143900 & -1.73642300 \\
\hline $\mathrm{H}$ & -1.22335300 & 3.41318600 & -1.52631700 \\
\hline $\mathrm{C}$ & -2.42845000 & 5.47273000 & 1.86877800 \\
\hline $\mathrm{H}$ & -3.02996500 & 6.30868300 & 1.52431300 \\
\hline $\mathrm{C}$ & -1.62611500 & 5.60701200 & 2.99734800 \\
\hline $\mathrm{H}$ & -1.59461200 & 6.54992200 & 3.53515800 \\
\hline $\mathrm{C}$ & -0.87068800 & 4.52381400 & 3.44173900 \\
\hline $\mathrm{H}$ & -0.24996300 & 4.61685200 & 4.32782800 \\
\hline $\mathrm{C}$ & -1.92234200 & -1.83325300 & 3.90084200 \\
\hline $\mathrm{H}$ & -1.48494200 & -0.84219100 & 3.99926600 \\
\hline $\mathrm{C}$ & -3.32930700 & -2.28232900 & -1.19194000 \\
\hline $\mathrm{H}$ & -2.32281900 & -2.31485500 & -1.60453100 \\
\hline $\mathrm{C}$ & -4.41746400 & -2.66167000 & -1.97771300 \\
\hline $\mathrm{H}$ & -4.24283600 & -3.00346600 & -2.99471500 \\
\hline $\mathrm{C}$ & 3.96582500 & 2.74847600 & 0.47381100 \\
\hline $\mathrm{H}$ & 3.90618400 & 3.18374400 & -0.52205800 \\
\hline $\mathrm{C}$ & -2.46932300 & 4.26681500 & 1.17349400 \\
\hline $\mathrm{H}$ & -3.10076600 & 4.18022900 & 0.29441800 \\
\hline $\mathrm{C}$ & -5.91406000 & -2.17510200 & $\begin{array}{c}-0.14589000 \\
\text { S88 }\end{array}$ \\
\hline
\end{tabular}




$\begin{array}{lrrr}\mathrm{H} & -6.91932100 & -2.13998400 & 0.26400400 \\ \mathrm{C} & 2.95230000 & -4.66127900 & -2.82134000 \\ \mathrm{H} & 2.83270100 & -5.73153900 & -2.68394700 \\ \mathrm{C} & -2.86528500 & 3.17720200 & -2.88071800 \\ \mathrm{H} & -2.48678600 & 3.92990300 & -3.56691700 \\ \mathrm{H} & 0.27533600 & 0.96235300 & 1.93439800 \\ \mathrm{H} & -0.75845600 & -0.04721500 & -1.33129900 \\ \mathrm{H} & -0.20404100 & -2.01630400 & -2.00134300 \\ \mathrm{H} & -0.86419700 & 0.01401800 & -2.12161900 \\ \mathrm{C} & 2.15555200 & 0.37315000 & -1.40104900 \\ \mathrm{H} & 3.12362300 & 0.67640500 & -1.81443300 \\ \mathrm{H} & 1.41689500 & 0.33729000 & -2.21113300 \\ \mathrm{C} & -2.59668700 & 0.38753800 & 1.67988800 \\ \mathrm{H} & -3.67886000 & 0.54983300 & 1.67730800 \\ \mathrm{H} & -2.24857400 & 0.52838500 & 2.70817500 \\ \mathrm{C} & -2.20768500 & -0.47852300 & -4.17604600 \\ \mathrm{H} & -2.91453700 & -0.92948600 & -3.47623800 \\ \mathrm{H} & -2.38817900 & 0.60460200 & -4.19724200 \\ \mathrm{H} & -2.42645000 & -0.87360100 & -5.18124500 \\ \mathrm{C} & 0.10693800 & -0.00236000 & -4.53326600 \\ \mathrm{H} & -0.04174200 & 1.08053700 & -4.38202000 \\ \mathrm{H} & 1.12614400 & -0.27907000 & -4.24484900 \\ \mathrm{H} & 0.01942500 & -0.18945600 & -5.61588400 \\ \mathrm{~N} & -0.84958800 & -0.77623100 & -3.77817600 \\ \mathrm{H} & -0.47795200 & -2.14489000 & -3.26520200 \\ \mathrm{H} & 0.54966800 & -2.54820600 & -3.75618000 \\ \mathrm{H} & -1.41719000 & -2.91126900 & -3.30648600\end{array}$

TS $_{\text {iso3 }}$

$\begin{array}{lccc}\mathrm{Fe} & -0.76384600 & -1.82422100 & 0.18777800 \\ \mathrm{P} & 1.45639600 & -2.05893400 & -0.02821400 \\ \mathrm{P} & -2.58843500 & -0.55696000 & 0.03632200 \\ \mathrm{P} & -0.95346100 & 2.28192800 & -0.11285400 \\ \mathrm{P} & 2.24167400 & 1.02906600 & -0.54127900 \\ \mathrm{O} & -0.53429300 & -1.22771800 & 3.01894900 \\ \mathrm{O} & -1.67413900 & -4.56732600 & 0.62489200 \\ & & & \mathrm{~S} 89\end{array}$




\begin{tabular}{|c|c|c|c|}
\hline $\mathrm{C}$ & -0.61292900 & -1.43963700 & 1.86200100 \\
\hline $\mathrm{C}$ & 1.85755900 & -3.38222800 & 2.36290800 \\
\hline $\mathrm{H}$ & 0.88250500 & -3.81383600 & 2.13498400 \\
\hline $\mathrm{C}$ & -1.23433300 & 3.90149100 & -0.87713800 \\
\hline $\mathrm{C}$ & 3.77494800 & -3.21456300 & 3.81966600 \\
\hline $\mathrm{H}$ & 4.30003400 & -3.51244500 & 4.72506900 \\
\hline $\mathrm{C}$ & -3.74756900 & -0.57149400 & 1.47286500 \\
\hline $\mathrm{C}$ & 3.33077900 & 2.01931700 & -1.58780100 \\
\hline $\mathrm{C}$ & -3.76680600 & -1.01694600 & -1.31543000 \\
\hline $\mathrm{C}$ & -1.32359100 & -3.48900200 & 0.32981500 \\
\hline $\mathrm{C}$ & 3.67122400 & -1.90272700 & 1.79838100 \\
\hline $\mathrm{H}$ & 4.13510800 & -1.15275500 & 1.15945400 \\
\hline $\mathrm{C}$ & 1.89554400 & 0.78409500 & 2.16919400 \\
\hline $\mathrm{H}$ & 1.01023700 & 0.21826600 & 1.88874800 \\
\hline $\mathrm{C}$ & -0.73093700 & 2.60861300 & 1.64583200 \\
\hline $\mathrm{C}$ & 4.41517600 & 1.47727800 & -2.28263400 \\
\hline $\mathrm{H}$ & 4.62795900 & 0.41056800 & -2.22655300 \\
\hline B & 0.39441000 & 1.24644500 & -0.97873200 \\
\hline $\mathrm{C}$ & 2.41811600 & -2.44199600 & 1.48583300 \\
\hline $\mathrm{C}$ & 1.07662400 & -4.15349900 & -1.89009900 \\
\hline $\mathrm{H}$ & 0.01837400 & -3.91237000 & -1.79660400 \\
\hline $\mathrm{C}$ & -0.77638600 & 5.51332000 & -2.62284600 \\
\hline $\mathrm{H}$ & -0.23844500 & 5.79151600 & -3.52670100 \\
\hline $\mathrm{C}$ & 3.06344500 & 3.39183700 & -1.68014400 \\
\hline $\mathrm{H}$ & 2.21710300 & 3.81846500 & -1.13713300 \\
\hline $\mathrm{C}$ & 3.36476600 & -3.76156400 & -1.24297200 \\
\hline $\mathrm{H}$ & 4.10104400 & -3.23623900 & -0.63189700 \\
\hline $\mathrm{C}$ & 2.00666600 & -3.42885100 & -1.14086200 \\
\hline $\mathrm{C}$ & -5.55155900 & -0.79526800 & 3.61566000 \\
\hline $\mathrm{H}$ & -6.24815500 & -0.87940800 & 4.44742400 \\
\hline $\mathrm{C}$ & -4.75793400 & 0.38106400 & 1.66580200 \\
\hline $\mathrm{H}$ & -4.88148800 & 1.21516000 & 0.97592000 \\
\hline $\mathrm{C}$ & 2.53144900 & -3.76490000 & 3.51738400 \\
\hline $\mathrm{H}$ & 2.08019000 & -4.49637500 & 4.18448100 \\
\hline $\mathrm{C}$ & 2.69108400 & 1.36026300 & 1.17417500 \\
\hline $\mathrm{C}$ & -4.57151500 & -1.76331400 & $\begin{array}{c}3.42077800 \\
\text { S90 }\end{array}$ \\
\hline
\end{tabular}




\begin{tabular}{|c|c|c|c|}
\hline $\mathrm{H}$ & -4.49791000 & -2.61284100 & 4.09659300 \\
\hline $\mathrm{C}$ & 4.17264900 & 2.22358600 & 2.86696600 \\
\hline $\mathrm{H}$ & 5.06210700 & 2.78625200 & 3.14250200 \\
\hline $\mathrm{C}$ & 3.37949300 & 1.64141800 & 3.85498400 \\
\hline $\mathrm{H}$ & 3.65647800 & 1.74896100 & 4.90218600 \\
\hline $\mathrm{C}$ & 1.49560000 & -5.17236400 & -2.74298800 \\
\hline $\mathrm{H}$ & 0.75950500 & -5.73084200 & -3.31900900 \\
\hline $\mathrm{C}$ & -0.55251500 & 4.26690900 & -2.04338900 \\
\hline $\mathrm{H}$ & 0.16030400 & 3.58197700 & -2.50004500 \\
\hline $\mathrm{C}$ & 5.22297400 & 2.30241100 & -3.06102300 \\
\hline $\mathrm{H}$ & 6.06371400 & 1.87601700 & -3.60366200 \\
\hline $\mathrm{C}$ & 3.87453800 & 4.21208500 & -2.45490300 \\
\hline $\mathrm{H}$ & 3.66235100 & 5.27728200 & -2.52057300 \\
\hline $\mathrm{C}$ & -1.68470600 & 6.39630900 & -2.04698800 \\
\hline $\mathrm{H}$ & -1.85864400 & 7.36995500 & -2.50008300 \\
\hline $\mathrm{C}$ & -5.55549900 & -1.84355600 & -3.31694800 \\
\hline $\mathrm{H}$ & -6.24682400 & -2.16201400 & -4.09474700 \\
\hline $\mathrm{C}$ & 2.23984600 & 0.91856400 & 3.50698300 \\
\hline $\mathrm{H}$ & 1.61544700 & 0.44890700 & 4.26485300 \\
\hline $\mathrm{C}$ & -1.36768000 & 1.82671000 & 2.61287700 \\
\hline $\mathrm{H}$ & -2.03602000 & 1.01779000 & 2.32559200 \\
\hline $\mathrm{C}$ & -4.74292700 & -0.14156100 & -1.81099800 \\
\hline $\mathrm{H}$ & -4.83114500 & 0.87109200 & -1.41748400 \\
\hline $\mathrm{C}$ & -5.64645500 & 0.27525900 & 2.72994200 \\
\hline $\mathrm{H}$ & -6.42101900 & 1.02825000 & 2.86182200 \\
\hline $\mathrm{C}$ & 2.84729500 & -5.48543800 & -2.84766500 \\
\hline $\mathrm{H}$ & 3.17329000 & -6.28754000 & -3.50709900 \\
\hline $\mathrm{C}$ & 4.95498800 & 3.66590800 & -3.14667000 \\
\hline $\mathrm{H}$ & 5.58812600 & 4.30589800 & -3.75715400 \\
\hline $\mathrm{C}$ & 4.34268200 & -2.28243600 & 2.95697900 \\
\hline $\mathrm{H}$ & 5.31204100 & -1.84191600 & 3.18437900 \\
\hline $\mathrm{C}$ & -2.14661900 & 4.79742800 & -0.30055700 \\
\hline $\mathrm{H}$ & -2.66801000 & 4.52899600 & 0.61893500 \\
\hline $\mathrm{C}$ & 0.35668900 & 3.88027600 & 3.38807900 \\
\hline $\mathrm{H}$ & 1.03074300 & 4.67868400 & 3.69142800 \\
\hline $\mathrm{C}$ & -0.27973600 & 3.09531800 & $\begin{array}{c}4.35075200 \\
\text { S91 }\end{array}$ \\
\hline
\end{tabular}




\begin{tabular}{|c|c|c|c|}
\hline $\mathrm{H}$ & -0.10054700 & 3.28474200 & 5.40747700 \\
\hline $\mathrm{C}$ & -1.13952400 & 2.07237500 & 3.96406800 \\
\hline $\mathrm{H}$ & -1.63387000 & 1.45025400 & 4.70744600 \\
\hline $\mathrm{C}$ & -3.67892400 & -1.65512000 & 2.35793700 \\
\hline $\mathrm{H}$ & -2.92632000 & -2.42557200 & 2.20475800 \\
\hline $\mathrm{C}$ & -3.72236500 & -2.32132000 & -1.82001600 \\
\hline $\mathrm{H}$ & -2.97306400 & -3.01109500 & -1.43479900 \\
\hline $\mathrm{C}$ & -4.61066500 & -2.73230300 & -2.81058000 \\
\hline $\mathrm{H}$ & -4.56139300 & -3.75209000 & -3.18831400 \\
\hline $\mathrm{C}$ & 3.83664300 & 2.08513800 & 1.52261200 \\
\hline $\mathrm{H}$ & 4.46451900 & 2.53264900 & 0.75339300 \\
\hline $\mathrm{C}$ & 0.13565400 & 3.64021100 & 2.03922600 \\
\hline $\mathrm{H}$ & 0.63497200 & 4.25470900 & 1.28845400 \\
\hline $\mathrm{C}$ & -5.62219200 & -0.54768800 & -2.81056900 \\
\hline $\mathrm{H}$ & -6.36749400 & 0.14949100 & -3.18894100 \\
\hline $\mathrm{C}$ & 3.78259400 & -4.78058300 & -2.09152800 \\
\hline $\mathrm{H}$ & 4.83921700 & -5.03395200 & -2.15448800 \\
\hline $\mathrm{C}$ & -2.37038000 & 6.03745900 & -0.88653600 \\
\hline $\mathrm{H}$ & -3.07658400 & 6.72975800 & -0.43372500 \\
\hline $\mathrm{H}$ & -0.02442500 & 0.14921400 & -0.70539300 \\
\hline $\mathrm{H}$ & 0.25775100 & 1.48903200 & -2.17987400 \\
\hline $\mathrm{H}$ & -0.78567900 & -1.78795300 & -1.52922500 \\
\hline $\mathrm{N}$ & -0.40011300 & -0.58585300 & -3.46149700 \\
\hline B & 0.44331600 & 0.66015100 & -3.45850200 \\
\hline $\mathrm{H}$ & 1.62276100 & 0.49458900 & -3.42463500 \\
\hline $\mathrm{H}$ & 0.00593600 & 1.60280800 & -4.05190600 \\
\hline $\mathrm{H}$ & -0.58633100 & -1.15743100 & -2.27023500 \\
\hline $\mathrm{C}$ & 2.54534500 & -0.73919200 & -0.87839100 \\
\hline $\mathrm{H}$ & 3.60289600 & -0.97860900 & -0.72171600 \\
\hline $\mathrm{H}$ & 2.35312300 & -0.86877100 & -1.95180600 \\
\hline $\mathrm{C}$ & -2.49007300 & 1.32665400 & -0.36347600 \\
\hline $\mathrm{H}$ & -3.30009200 & 1.88345500 & 0.12160300 \\
\hline $\mathrm{H}$ & -2.66508000 & 1.41414700 & -1.44461100 \\
\hline $\mathrm{C}$ & 0.25552000 & -1.70721600 & -4.16707000 \\
\hline $\mathrm{H}$ & 1.23362900 & -1.90565100 & -3.71884000 \\
\hline $\mathrm{H}$ & -0.36241600 & -2.60670000 & $\begin{array}{c}-4.06210900 \\
\text { S92 }\end{array}$ \\
\hline
\end{tabular}




$\begin{array}{llll}\mathrm{H} & 0.38357600 & -1.48222600 & -5.23228900 \\ \mathrm{C} & -1.76209500 & -0.37767800 & -3.98932300 \\ \mathrm{H} & -2.35322700 & -1.29231100 & -3.87808100 \\ \mathrm{H} & -2.25520600 & 0.42291600 & -3.42951200 \\ \mathrm{H} & -1.72251000 & -0.09239600 & -5.04711000\end{array}$

TS iso4

$\begin{array}{lrrr}\mathrm{Fe} & -0.82422000 & 0.44113100 & 0.39395700 \\ \mathrm{P} & 1.01106400 & -0.86553500 & 0.85484300 \\ \mathrm{P} & -2.88552000 & 1.29312400 & -0.23463200 \\ \mathrm{P} & -1.80016700 & -0.98769100 & -1.20060900 \\ \mathrm{P} & 3.52971600 & 0.45058900 & -0.62095500 \\ \mathrm{O} & 0.30420400 & 3.01481800 & 1.22837400 \\ \mathrm{O} & -1.85211500 & -0.37575200 & 3.00214000 \\ \mathrm{C} & -0.07680000 & 1.98194900 & 0.83819800 \\ \mathrm{C} & 2.70731900 & -1.55611400 & 2.97496600 \\ \mathrm{H} & 2.95829700 & -2.42597800 & 2.37004500 \\ \mathrm{C} & -2.96557300 & -2.30260600 & -0.71472100 \\ \mathrm{C} & 2.95361900 & -0.31115800 & 5.02424100 \\ \mathrm{H} & 3.40751000 & -0.19321100 & 6.00397300 \\ \mathrm{C} & -3.26917300 & 3.03292100 & -0.57959200 \\ \mathrm{C} & 5.09983400 & -0.31306800 & -1.14321600 \\ \mathrm{C} & -4.28606100 & 0.75581100 & 0.80070700 \\ \mathrm{C} & -1.46128600 & -0.08763800 & 1.94343300 \\ \mathrm{C} & 1.44010000 & 0.46666100 & 3.31369300 \\ \mathrm{H} & 0.70941300 & 1.19256600 & 2.97501500 \\ \mathrm{C} & 3.37742000 & 2.66520700 & 1.02746700 \\ \mathrm{H} & 2.73370700 & 3.07193200 & 0.25120100 \\ \mathrm{C} & -0.83624800 & -1.64231600 & -2.61083400 \\ \mathrm{C} & 5.12787700 & -1.55117000 & -1.79895500 \\ \mathrm{H} & 4.21495200 & -2.12784200 & -1.93267200 \\ \mathrm{C} & 2.91088600 & 1.51319800 & -2.04711400 \\ \mathrm{C} & 1.77590800 & -0.61809900 & 2.50238300 \\ \mathrm{H} & 0.55212700 & -3.46301900 & -0.17447300 \\ \mathrm{C} & 0.91667200 & -3.09095000 & -1.12846900 \\ \mathrm{H} & -4.90478200 & -3.67585400 & -1.19747600\end{array}$




\begin{tabular}{|c|c|c|c|}
\hline $\mathrm{H}$ & -5.66893400 & -4.00890500 & -1.89384000 \\
\hline $\mathrm{C}$ & 6.28816800 & 0.41873100 & -1.01297600 \\
\hline $\mathrm{H}$ & 6.27600400 & 1.38849400 & -0.52098500 \\
\hline $\mathrm{C}$ & 0.08137000 & -3.16068300 & 2.17361300 \\
\hline $\mathrm{H}$ & 0.07297300 & -2.53750700 & 3.06431400 \\
\hline $\mathrm{C}$ & 0.54298000 & -2.63728000 & 0.95666200 \\
\hline $\mathrm{C}$ & -3.87968900 & 5.69859900 & -1.11387600 \\
\hline $\mathrm{H}$ & -4.11097600 & 6.73775500 & -1.32780900 \\
\hline $\mathrm{C}$ & -2.73124200 & 4.04660400 & 0.21655000 \\
\hline $\mathrm{H}$ & -2.06404900 & 3.80155700 & 1.03809000 \\
\hline $\mathrm{C}$ & 3.28958400 & -1.40487800 & 4.22713500 \\
\hline $\mathrm{H}$ & 4.00117500 & -2.14381300 & 4.58398700 \\
\hline $\mathrm{C}$ & 3.91442000 & 1.38371400 & 0.87502100 \\
\hline $\mathrm{C}$ & -4.42894100 & 4.68830700 & -1.90044500 \\
\hline $\mathrm{H}$ & -5.08741300 & 4.93736100 & -2.72717200 \\
\hline $\mathrm{C}$ & 5.03347200 & 1.60366000 & 3.00425400 \\
\hline $\mathrm{H}$ & 5.67994200 & 1.19151900 & 3.77366200 \\
\hline $\mathrm{C}$ & 4.48730900 & 2.87868300 & 3.15549900 \\
\hline $\mathrm{H}$ & 4.71291100 & 3.46066300 & 4.04455000 \\
\hline $\mathrm{C}$ & 0.09221100 & -4.77501600 & -0.09600000 \\
\hline $\mathrm{H}$ & 0.10141000 & -5.39810900 & -0.98605700 \\
\hline $\mathrm{C}$ & -3.95949900 & -2.74160700 & -1.60508300 \\
\hline $\mathrm{H}$ & -4.00364400 & -2.34043800 & -2.61666900 \\
\hline $\mathrm{C}$ & 6.32751900 & -2.05804400 & -2.28750700 \\
\hline $\mathrm{H}$ & 6.33938900 & -3.02270600 & -2.78633300 \\
\hline $\mathrm{C}$ & 7.48390200 & -0.09425100 & -1.50197600 \\
\hline $\mathrm{H}$ & 8.40004300 & 0.47736600 & -1.38743300 \\
\hline $\mathrm{C}$ & -4.87575100 & -4.17666700 & 0.10422800 \\
\hline $\mathrm{H}$ & -5.62053800 & -4.89995300 & 0.42331100 \\
\hline $\mathrm{C}$ & -6.39799000 & 0.01958100 & 2.48109600 \\
\hline $\mathrm{H}$ & -7.21570300 & -0.26903000 & 3.13477900 \\
\hline $\mathrm{C}$ & 3.65936500 & 3.40770300 & 2.17049300 \\
\hline $\mathrm{H}$ & 3.23442900 & 4.40029400 & 2.28568800 \\
\hline $\mathrm{C}$ & -1.12980400 & -2.84634800 & -3.26310600 \\
\hline $\mathrm{H}$ & -1.99500900 & -3.43323600 & -2.96928200 \\
\hline $\mathrm{C}$ & -5.25217000 & -0.15487400 & $\begin{array}{c}0.36535500 \\
\text { S94 }\end{array}$ \\
\hline
\end{tabular}




\begin{tabular}{|c|c|c|c|}
\hline $\mathrm{H}$ & -5.19206200 & -0.59709900 & -0.62406700 \\
\hline $\mathrm{C}$ & -3.03798400 & 5.37729000 & -0.05268400 \\
\hline $\mathrm{H}$ & -2.61385400 & 6.16318600 & 0.56553700 \\
\hline $\mathrm{C}$ & -0.36973700 & -5.28346400 & 1.11425100 \\
\hline $\mathrm{H}$ & -0.72718400 & -6.30711100 & 1.17470600 \\
\hline $\mathrm{C}$ & 7.50699500 & -1.33353700 & -2.13725800 \\
\hline $\mathrm{H}$ & 8.44202500 & -1.73223300 & -2.51841600 \\
\hline $\mathrm{C}$ & 2.03133600 & 0.62223700 & 4.56556100 \\
\hline $\mathrm{H}$ & 1.76384900 & 1.47547900 & 5.18173400 \\
\hline $\mathrm{C}$ & -2.94907300 & -2.80939400 & 0.58442700 \\
\hline $\mathrm{H}$ & -2.19328100 & -2.46617700 & 1.28215900 \\
\hline $\mathrm{C}$ & 1.14578600 & -1.40750400 & -3.98955700 \\
\hline $\mathrm{H}$ & 2.03432000 & -0.84181500 & -4.26072400 \\
\hline $\mathrm{C}$ & 0.84065500 & -2.60039800 & -4.64117500 \\
\hline $\mathrm{H}$ & 1.48921600 & -2.97444700 & -5.42806000 \\
\hline $\mathrm{C}$ & -0.29435900 & -3.31836300 & -4.27257300 \\
\hline $\mathrm{H}$ & -0.53243500 & -4.25448600 & -4.76960900 \\
\hline $\mathrm{C}$ & -4.12795700 & 3.35762200 & -1.63507900 \\
\hline $\mathrm{H}$ & -4.54958400 & 2.57371100 & -2.26107500 \\
\hline $\mathrm{C}$ & -4.39698600 & 1.30475100 & 2.08609000 \\
\hline $\mathrm{H}$ & -3.65387400 & 2.01824800 & 2.43893400 \\
\hline $\mathrm{C}$ & -5.44704300 & 0.93989600 & 2.91870900 \\
\hline $\mathrm{H}$ & -5.52164500 & 1.37212600 & 3.91192000 \\
\hline $\mathrm{C}$ & 4.74979000 & 0.85629600 & 1.86938200 \\
\hline $\mathrm{H}$ & 5.17990100 & -0.13628100 & 1.75007100 \\
\hline $\mathrm{C}$ & 0.31787000 & -0.93617700 & -2.97612600 \\
\hline $\mathrm{H}$ & 0.57533700 & -0.02024700 & -2.44513000 \\
\hline $\mathrm{C}$ & -6.29891800 & -0.52486400 & 1.20525000 \\
\hline $\mathrm{H}$ & -7.03528600 & -1.24263000 & 0.85497200 \\
\hline $\mathrm{C}$ & -0.36521100 & -4.47563400 & 2.24974000 \\
\hline $\mathrm{H}$ & -0.71442800 & -4.86834500 & 3.20041600 \\
\hline $\mathrm{C}$ & -3.89779900 & -3.74250100 & 0.99284000 \\
\hline $\mathrm{H}$ & -3.87279200 & -4.12241900 & 2.01032600 \\
\hline $\mathrm{H}$ & 3.61036800 & 2.48890600 & -2.13690300 \\
\hline $\mathrm{C}$ & -2.96415300 & 0.32947600 & -1.79540100 \\
\hline $\mathrm{H}$ & -2.47254800 & 0.91927200 & $\begin{array}{c}-2.57513300 \\
\text { S95 }\end{array}$ \\
\hline
\end{tabular}




$\begin{array}{lrrr}\mathrm{H} & -3.94443400 & -0.00476700 & -2.14862900 \\ \mathrm{C} & 2.50404900 & -1.01814200 & -0.22925100 \\ \mathrm{H} & 3.14907400 & -1.78826200 & 0.21116600 \\ \mathrm{H} & 2.16399700 & -1.39506700 & -1.20111200 \\ \mathrm{H} & 2.79104300 & 0.82530900 & -3.02543100 \\ \mathrm{~N} & 0.79174700 & 2.99735600 & -2.72480200 \\ \mathrm{~B} & -0.61093000 & 2.37921400 & -2.65296800 \\ \mathrm{H} & -0.65556500 & 1.69744200 & -1.59554700 \\ \mathrm{H} & -1.42130300 & 3.26068800 & -2.49514500 \\ \mathrm{H} & 1.60186400 & 2.18392400 & -2.12131500 \\ \mathrm{H} & -0.79306000 & 1.65496700 & -3.60436000 \\ \mathrm{C} & 1.38192300 & 3.10437700 & -4.06243500 \\ \mathrm{H} & 2.42811000 & 3.41888400 & -3.99981700 \\ \mathrm{H} & 1.31640100 & 2.13542000 & -4.55899500 \\ \mathrm{H} & 0.81352600 & 3.83680500 & -4.64283000 \\ \mathrm{C} & 0.96923600 & 4.23581600 & -1.95559900 \\ \mathrm{H} & 0.50333600 & 4.12288500 & -0.97599600 \\ \mathrm{H} & 2.03537600 & 4.46181900 & -1.83974000 \\ \mathrm{H} & 0.48012000 & 5.06032900 & -2.48166300\end{array}$

TS $_{\text {iso5 }}$

$\begin{array}{lrrr}\mathrm{Fe} & 0.25069400 & -1.44907000 & -0.19439500 \\ \mathrm{P} & 2.36490400 & -0.85417800 & -0.90599400 \\ \mathrm{P} & -1.76898100 & -1.72755500 & 0.61352900 \\ \mathrm{P} & -1.83415700 & 1.19775200 & 0.36788800 \\ \mathrm{P} & 1.46810700 & 1.71618900 & -0.39185600 \\ \mathrm{O} & 0.09894500 & -3.86243400 & -1.81557600 \\ \mathrm{~N} & 2.53894700 & 0.77389200 & -1.15321800 \\ \mathrm{~N} & -2.48414700 & -0.19517600 & 1.05619800 \\ \mathrm{C} & 4.92679000 & -1.88132200 & -0.14814000 \\ \mathrm{H} & 5.10595400 & -2.10821000 & -1.19605000 \\ \mathrm{C} & -2.87130100 & 1.66929900 & -1.04803600 \\ \mathrm{C} & 5.72427900 & -1.79905700 & 2.13220900 \\ \mathrm{H} & 6.51322800 & -1.97267700 & 2.85945000 \\ \mathrm{C} & -1.93989700 & -2.67639300 & 2.15589900 \\ \mathrm{C} & 1.07161400 & 3.11819400 & -1.48333300\end{array}$




\begin{tabular}{|c|c|c|c|}
\hline $\mathrm{C}$ & -3.09139500 & -2.42737700 & -0.43864800 \\
\hline $\mathrm{C}$ & 0.21362700 & -2.91790400 & -1.13543200 \\
\hline $\mathrm{C}$ & 3.49912900 & -1.04094900 & 1.60253100 \\
\hline $\mathrm{H}$ & 2.55319900 & -0.60933200 & 1.92391900 \\
\hline $\mathrm{C}$ & 3.56025700 & 2.19240800 & 1.35850100 \\
\hline $\mathrm{H}$ & 4.13180100 & 1.62276200 & 0.62943700 \\
\hline $\mathrm{C}$ & -2.02235800 & 2.48214900 & 1.63025900 \\
\hline $\mathrm{C}$ & 0.87845800 & 2.82789400 & -2.84092800 \\
\hline $\mathrm{H}$ & 1.05689600 & 1.81682900 & -3.19672500 \\
\hline B & -0.09546900 & 0.64535100 & -0.13986200 \\
\hline $\mathrm{C}$ & 3.69656400 & -1.34952500 & 0.25175900 \\
\hline $\mathrm{C}$ & 3.05149100 & -2.97429600 & -2.61312600 \\
\hline $\mathrm{H}$ & 2.96759100 & -3.60857500 & -1.73224900 \\
\hline $\mathrm{C}$ & -4.72776700 & 1.23041700 & -2.53711200 \\
\hline $\mathrm{H}$ & -5.59419200 & 0.62940300 & -2.79913400 \\
\hline $\mathrm{C}$ & 0.81725400 & 4.41240200 & -1.01719100 \\
\hline $\mathrm{H}$ & 0.97853000 & 4.65462000 & 0.03074900 \\
\hline $\mathrm{C}$ & 2.95283800 & -0.78950900 & -3.63387700 \\
\hline $\mathrm{H}$ & 2.80655000 & 0.28201000 & -3.52596500 \\
\hline $\mathrm{C}$ & 2.85061900 & -1.59362400 & -2.49645100 \\
\hline $\mathrm{C}$ & -2.02831400 & -4.22747800 & 4.47873900 \\
\hline $\mathrm{H}$ & -2.05851100 & -4.82879600 & 5.38299700 \\
\hline $\mathrm{C}$ & -1.60990200 & -4.03805700 & 2.11140900 \\
\hline $\mathrm{H}$ & -1.30338100 & -4.48870100 & 1.16827600 \\
\hline $\mathrm{C}$ & 5.93059700 & -2.11112500 & 0.79087300 \\
\hline $\mathrm{H}$ & 6.88186500 & -2.52707700 & 0.46879000 \\
\hline $\mathrm{C}$ & 2.20348700 & 2.42940800 & 1.11471200 \\
\hline $\mathrm{C}$ & -2.34952600 & -2.87444900 & 4.53014800 \\
\hline $\mathrm{H}$ & -2.62288600 & -2.41270100 & 5.47500200 \\
\hline $\mathrm{C}$ & 2.05276800 & 3.61484600 & 3.22097400 \\
\hline $\mathrm{H}$ & 1.45896900 & 4.16330300 & 3.94778500 \\
\hline $\mathrm{C}$ & 3.40244500 & 3.36684900 & 3.46024500 \\
\hline $\mathrm{H}$ & 3.86515500 & 3.72156400 & 4.37744000 \\
\hline $\mathrm{C}$ & 3.35458400 & -3.53898700 & -3.84636600 \\
\hline $\mathrm{H}$ & 3.50825600 & -4.61168200 & -3.92639400 \\
\hline $\mathrm{C}$ & -3.99972600 & 0.91842200 & $\begin{array}{c}-1.39266100 \\
\text { S97 }\end{array}$ \\
\hline
\end{tabular}




\begin{tabular}{|c|c|c|c|}
\hline $\mathrm{H}$ & -4.30108600 & 0.06700800 & -0.78717100 \\
\hline $\mathrm{C}$ & 0.43359700 & 3.81494600 & -3.71255500 \\
\hline $\mathrm{H}$ & 0.28414700 & 3.57792400 & -4.76264800 \\
\hline $\mathrm{C}$ & 0.36272000 & 5.39695500 & -1.89319900 \\
\hline $\mathrm{H}$ & 0.17025300 & 6.40037600 & -1.52226500 \\
\hline $\mathrm{C}$ & -4.33925500 & 2.29506600 & -3.34416800 \\
\hline $\mathrm{H}$ & -4.90688200 & 2.53519000 & -4.23874200 \\
\hline $\mathrm{C}$ & -5.21967000 & -3.18538800 & -2.08301500 \\
\hline $\mathrm{H}$ & -6.04776800 & -3.47909900 & -2.72258200 \\
\hline $\mathrm{C}$ & 4.15411200 & 2.65759800 & 2.52800900 \\
\hline $\mathrm{H}$ & 5.20560500 & 2.45435700 & 2.71374700 \\
\hline $\mathrm{C}$ & -2.32246400 & 3.81123600 & 1.30838800 \\
\hline $\mathrm{H}$ & -2.56782400 & 4.08727600 & 0.28630800 \\
\hline $\mathrm{C}$ & -4.29435400 & -2.87903700 & 0.12196400 \\
\hline $\mathrm{H}$ & -4.39679700 & -2.93925300 & 1.20456000 \\
\hline $\mathrm{C}$ & -1.65849000 & -4.80940200 & 3.26541400 \\
\hline $\mathrm{H}$ & -1.40032900 & -5.86359700 & 3.22171000 \\
\hline $\mathrm{C}$ & 3.45201900 & -2.73064600 & -4.97802600 \\
\hline $\mathrm{H}$ & 3.68206000 & -3.17356800 & -5.94363100 \\
\hline $\mathrm{C}$ & 0.16842100 & 5.09981100 & -3.24008600 \\
\hline $\mathrm{H}$ & -0.18399900 & 5.86971000 & -3.92180600 \\
\hline $\mathrm{C}$ & 4.50600400 & -1.25525200 & 2.53621800 \\
\hline $\mathrm{H}$ & 4.33749500 & -0.98332600 & 3.57651100 \\
\hline $\mathrm{C}$ & -2.47187500 & 2.72478600 & -1.87918900 \\
\hline $\mathrm{H}$ & -1.57185900 & 3.29253100 & -1.65297800 \\
\hline $\mathrm{C}$ & -1.71585800 & 3.11434400 & 3.94238100 \\
\hline $\mathrm{H}$ & -1.46638300 & 2.83712000 & 4.96291600 \\
\hline $\mathrm{C}$ & -2.00354600 & 4.43950300 & 3.61833900 \\
\hline $\mathrm{H}$ & -1.98947200 & 5.20440100 & 4.39017700 \\
\hline $\mathrm{C}$ & -2.30801900 & 4.78476800 & 2.30349500 \\
\hline $\mathrm{H}$ & -2.54008200 & 5.81508500 & 2.04957700 \\
\hline $\mathrm{C}$ & -2.30663700 & -2.09870300 & 3.37257900 \\
\hline $\mathrm{H}$ & -2.52779400 & -1.03627000 & 3.42216400 \\
\hline $\mathrm{C}$ & -2.95937800 & -2.37066500 & -1.83138000 \\
\hline $\mathrm{H}$ & -2.03601600 & -2.00808800 & -2.27555200 \\
\hline $\mathrm{C}$ & -4.02345500 & -2.74910500 & $\begin{array}{c}-2.64598400 \\
\text { S98 }\end{array}$ \\
\hline
\end{tabular}




$\begin{array}{lrrr}\mathrm{H} & -3.91479400 & -2.69681400 & -3.72553000 \\ \mathrm{C} & 1.45535100 & 3.14573000 & 2.05633900 \\ \mathrm{H} & 0.39871400 & 3.33553300 & 1.88559800 \\ \mathrm{C} & -1.71945100 & 2.13744000 & 2.95439400 \\ \mathrm{H} & -1.44683000 & 1.11384600 & 3.20131900 \\ \mathrm{C} & -5.35396100 & -3.25407500 & -0.69702700 \\ \mathrm{H} & -6.28289200 & -3.60472400 & -0.25536800 \\ \mathrm{C} & 3.25231500 & -1.35747100 & -4.86918200 \\ \mathrm{H} & 3.32957000 & -0.72486900 & -5.74964600 \\ \mathrm{C} & -3.21005000 & 3.04108400 & -3.01319900 \\ \mathrm{H} & -2.88606800 & 3.86136100 & -3.64794500 \\ \mathrm{H} & 0.42737500 & -0.10711100 & 2.34390300 \\ \mathrm{H} & -3.46717200 & -0.18404100 & 1.31646700 \\ \mathrm{C} & -0.28362800 & -0.24308900 & -1.45727000 \\ \mathrm{O} & -0.58598000 & -0.14836300 & -2.62603800 \\ \mathrm{H} & 1.05563900 & -2.52046100 & 1.05350500 \\ \mathrm{H} & -0.31348100 & -0.17626500 & 4.21825900 \\ \mathrm{~B} & 0.73179500 & -0.46435200 & 3.67609400 \\ \mathrm{H} & 0.30478700 & -0.09649500 & 1.34804900 \\ \mathrm{~N} & 1.04615300 & -1.90802600 & 3.64509100 \\ \mathrm{H} & 1.99236900 & -2.21206500 & 3.81706500 \\ \mathrm{H} & 0.38336100 & -2.56253400 & 4.03477400 \\ \mathrm{H} & 1.66337400 & 0.28308700 & 3.86617500 \\ \mathrm{H} & 0.95923600 & -2.22571800 & 1.80062800\end{array}$

TS $_{\text {iso6 }}$

$\begin{array}{lrrc}\mathrm{Fe} & -0.33209400 & -1.55452900 & 0.31113200 \\ \mathrm{P} & 1.73673800 & -1.78178400 & -0.44786800 \\ \mathrm{P} & -2.50038000 & -0.86828300 & 0.49101700 \\ \mathrm{P} & -1.50160200 & 1.74209000 & 0.49435200 \\ \mathrm{P} & 1.83795300 & 1.08310400 & -0.36168400 \\ \mathrm{O} & 0.10375400 & -1.43576500 & 3.22257300 \\ \mathrm{O} & -1.12482400 & -4.34822000 & 0.16502700 \\ \mathrm{~N} & 2.35116800 & -0.30990500 & -1.19871500 \\ \mathrm{C} & -0.06484700 & -1.47287600 & 2.07261900 \\ & & & \mathrm{~S} 99\end{array}$




\begin{tabular}{|c|c|c|c|}
\hline $\mathrm{N}$ & -2.71567100 & 0.73468100 & 0.83063100 \\
\hline $\mathrm{C}$ & 2.75193800 & -2.93658000 & 1.87060700 \\
\hline $\mathrm{H}$ & 1.75049500 & -3.34972900 & 1.96969800 \\
\hline $\mathrm{C}$ & -1.93941800 & 2.77228600 & -0.94474100 \\
\hline $\mathrm{C}$ & 4.99730700 & -2.67423100 & 2.70961800 \\
\hline $\mathrm{H}$ & 5.74898500 & -2.87978800 & 3.46695800 \\
\hline $\mathrm{C}$ & -3.38491700 & -1.71533500 & 1.84278400 \\
\hline $\mathrm{C}$ & 2.06188400 & 2.48487200 & -1.49511000 \\
\hline $\mathrm{C}$ & -3.59639100 & -1.20423300 & -0.94178300 \\
\hline $\mathrm{C}$ & -0.79134200 & -3.23654800 & 0.25403000 \\
\hline $\mathrm{C}$ & 4.34516400 & -1.59477800 & 0.65332300 \\
\hline $\mathrm{H}$ & 4.57593900 & -0.94767700 & -0.18884400 \\
\hline $\mathrm{C}$ & 2.90588400 & 1.11100900 & 2.26337200 \\
\hline $\mathrm{H}$ & 1.93322800 & 0.78073200 & 2.61465300 \\
\hline $\mathrm{C}$ & -1.35776300 & 2.94944900 & 1.84534900 \\
\hline $\mathrm{C}$ & 1.94207700 & 2.35810100 & -2.88432600 \\
\hline $\mathrm{H}$ & 1.76812500 & 1.38846000 & -3.34566900 \\
\hline B & 0.12141200 & 0.69005000 & 0.42591000 \\
\hline $\mathrm{C}$ & 3.05185200 & -2.11677400 & 0.77765800 \\
\hline $\mathrm{C}$ & 3.42613700 & -3.22481000 & -2.15311800 \\
\hline $\mathrm{H}$ & 4.23759900 & -2.71568500 & -1.64068800 \\
\hline $\mathrm{C}$ & -1.47629100 & 4.60004600 & -2.45993800 \\
\hline $\mathrm{H}$ & -0.86953900 & 5.45830300 & -2.73687300 \\
\hline $\mathrm{C}$ & 2.29017800 & 3.75345600 & -0.94003500 \\
\hline $\mathrm{H}$ & 2.41078900 & 3.86439100 & 0.13569600 \\
\hline $\mathrm{C}$ & 1.06809500 & -3.65209400 & -2.44142500 \\
\hline $\mathrm{H}$ & 0.03534700 & -3.44734500 & -2.18309200 \\
\hline $\mathrm{C}$ & 2.09815100 & -2.98187700 & -1.77646900 \\
\hline $\mathrm{C}$ & -4.63093300 & -3.04435300 & 3.96322900 \\
\hline $\mathrm{H}$ & -5.11846000 & -3.55938800 & 4.78652400 \\
\hline $\mathrm{C}$ & -3.48848500 & -1.10308300 & 3.09493700 \\
\hline $\mathrm{H}$ & -3.08936600 & -0.09928900 & 3.22529200 \\
\hline $\mathrm{C}$ & 3.71971300 & -3.21511800 & 2.83046100 \\
\hline $\mathrm{H}$ & 3.47121900 & -3.84741300 & 3.67795200 \\
\hline $\mathrm{C}$ & 3.11682800 & 1.37279500 & 0.90561900 \\
\hline $\mathrm{C}$ & -4.53016900 & -3.66018200 & $\begin{array}{c}2.71732700 \\
\text { S100 }\end{array}$ \\
\hline
\end{tabular}




\begin{tabular}{|c|c|c|c|}
\hline $\mathrm{H}$ & -4.94027000 & -4.65491300 & 2.56534300 \\
\hline $\mathrm{C}$ & 5.42654200 & 1.92580900 & 1.39521100 \\
\hline $\mathrm{H}$ & 6.40770200 & 2.24314300 & 1.05302200 \\
\hline $\mathrm{C}$ & 5.20514400 & 1.66355200 & 2.74701100 \\
\hline $\mathrm{H}$ & 6.01542200 & 1.77506200 & 3.46239700 \\
\hline $\mathrm{C}$ & 3.71408100 & -4.11752200 & -3.17755500 \\
\hline $\mathrm{H}$ & 4.74723400 & -4.29509600 & -3.46318200 \\
\hline $\mathrm{C}$ & -1.17449300 & 3.88637500 & -1.30456800 \\
\hline $\mathrm{H}$ & -0.33781200 & 4.19815900 & -0.68176200 \\
\hline $\mathrm{C}$ & 2.03166600 & 3.48635500 & -3.69560400 \\
\hline $\mathrm{H}$ & 1.93110300 & 3.37206400 & -4.77075400 \\
\hline $\mathrm{C}$ & 2.37833900 & 4.87471000 & -1.75685200 \\
\hline $\mathrm{H}$ & 2.55459900 & 5.85031600 & -1.31286700 \\
\hline $\mathrm{C}$ & -2.55116500 & 4.21226800 & -3.25799400 \\
\hline $\mathrm{H}$ & -2.78350100 & 4.76725300 & -4.16275400 \\
\hline $\mathrm{C}$ & -5.31040800 & -1.38717300 & -3.14878000 \\
\hline $\mathrm{H}$ & -5.97353800 & -1.44807600 & -4.00774600 \\
\hline $\mathrm{C}$ & 3.94744900 & 1.25519500 & 3.17663300 \\
\hline $\mathrm{H}$ & 3.77052000 & 1.03792300 & 4.22584100 \\
\hline $\mathrm{C}$ & -0.12896400 & 3.54707700 & 2.13961900 \\
\hline $\mathrm{H}$ & 0.76065900 & 3.25507900 & 1.58001700 \\
\hline $\mathrm{C}$ & -3.13116500 & -1.69921200 & -2.16070500 \\
\hline $\mathrm{H}$ & -2.08845800 & -1.98891800 & -2.26879300 \\
\hline $\mathrm{C}$ & -4.11019600 & -1.76626700 & 4.14933500 \\
\hline $\mathrm{H}$ & -4.19221500 & -1.28125200 & 5.11856800 \\
\hline $\mathrm{C}$ & 2.68066400 & -4.78528700 & -3.83147100 \\
\hline $\mathrm{H}$ & 2.90625700 & -5.48607700 & -4.63080700 \\
\hline $\mathrm{C}$ & 2.24459300 & 4.74322100 & -3.13764300 \\
\hline $\mathrm{H}$ & 2.31114800 & 5.61965700 & -3.77631700 \\
\hline $\mathrm{C}$ & 5.30952900 & -1.86840500 & 1.61695100 \\
\hline $\mathrm{H}$ & 6.30361000 & -1.43938100 & 1.51976200 \\
\hline $\mathrm{C}$ & -3.02767500 & 2.39832300 & -1.73957400 \\
\hline $\mathrm{H}$ & -3.62681300 & 1.53777500 & -1.44735800 \\
\hline $\mathrm{C}$ & -2.38137600 & 4.22759700 & 3.62256800 \\
\hline $\mathrm{H}$ & -3.25844600 & 4.48574600 & 4.20954300 \\
\hline $\mathrm{C}$ & -1.15633100 & 4.83165800 & $\begin{array}{c}3.89898000 \\
\text { S101 }\end{array}$ \\
\hline
\end{tabular}




$\begin{array}{lrrr}\mathrm{H} & -1.07863600 & 5.56215400 & 4.69954200 \\ \mathrm{C} & -0.02749000 & 4.48956700 & 3.15828100 \\ \mathrm{H} & 0.93319000 & 4.94568900 & 3.38225400 \\ \mathrm{C} & -3.90990000 & -3.00007700 & 1.66195500 \\ \mathrm{H} & -3.84278400 & -3.48094200 & 0.68803200 \\ \mathrm{C} & -4.94112500 & -0.81484600 & -0.83385900 \\ \mathrm{H} & -5.30767900 & -0.41963200 & 0.11151600 \\ \mathrm{C} & -5.79107900 & -0.90850600 & -1.92877700 \\ \mathrm{H} & -6.82987300 & -0.60216900 & -1.83496300 \\ \mathrm{C} & 4.39212000 & 1.77783700 & 0.48110800 \\ \mathrm{H} & 4.57016200 & 1.97140300 & -0.57461300 \\ \mathrm{C} & -2.48506600 & 3.29061600 & 2.59908600 \\ \mathrm{H} & -3.43106500 & 2.79908600 & 2.38297900 \\ \mathrm{C} & -3.98227300 & -1.78216300 & -3.26238200 \\ \mathrm{H} & -3.59263500 & -2.14637500 & -4.20893200 \\ \mathrm{C} & 1.36060400 & -4.55160500 & -3.46157100 \\ \mathrm{H} & 0.54989000 & -5.05996100 & -3.97457100 \\ \mathrm{C} & -3.32973600 & 3.11656900 & -2.89269800 \\ \mathrm{H} & -4.17251500 & 2.81451200 & -3.50858100 \\ \mathrm{H} & -0.34177700 & 0.69467500 & 1.60411600 \\ \mathrm{H} & -0.27622600 & -0.02755300 & -0.84535400 \\ \mathrm{H} & 2.00941300 & -0.31498900 & -2.18322600 \\ \mathrm{H} & -0.54321500 & -1.64855600 & -1.19079400 \\ \mathrm{H} & -0.47808800 & 0.43346700 & -2.41656000 \\ \mathrm{H} & -0.38076100 & 1.43225000 & -2.59454500 \\ \mathrm{H} & -1.48878400 & 0.30057100 & -2.39619700 \\ \mathrm{H} & -0.15464300 & 0.13565100 & -4.68487900 \\ \mathrm{H} & -30121100 & -0.57132900 & -3.54362400 \\ \mathrm{H} & -1.53312600 & -3.58636900\end{array}$

(1) Nöth, H.; Meinel, L. Amino-phosphane. VIII. Über Ein Octaphenyltetraphosphornitridchlorid Und Ein Tetraphenyldiphosphin-Ndiphenylphosphino-imid. ZAAC - J. Inorg. Gen. Chem. 1967, 349 (5-6), 225-240. 
(2) Sommer, K. Zur Spaltung Tertiärer Phosphine. I. ZAAC - J. Inorg. Gen. Chem. 1970, $376(1), 37-43$.

(3) Vondung, L.; Frank, N.; Fritz, M.; Alig, L.; Langer, R. Phosphine-Stabilized Borylenes and Boryl Anions as Ligands? Redox Reactivity in Boron-Based Pincer Complexes. Angew. Chem. Int. Ed. 2016, 55 (46), 14450-14454.

(4) Sheldrick, G. M. A Short History of SHELX. Acta Crystallogr. Sect. A Found. Crystallogr. 2008, 64 (1), 112-122.

(5) Sheldrick, G. M. SHELXT - Integrated Space-Group and Crystal-Structure Determination. Acta Crystallogr. Sect. A Found. Crystallogr. 2015, 71 (1), 3-8.

(6) Dolomanov, O. V.; Bourhis, L. J.; Gildea, R. J.; Howard, J. A. K.; Puschmann, H. OLEX2: A Complete Structure Solution, Refinement and Analysis Program. J. Appl. Crystallogr. 2009, 42 (2), 339-341.

(7) Bourhis, L. J.; Dolomanov, O. V.; Gildea, R. J.; Howard, J. A. K.; Puschmann, H. The Anatomy of a Comprehensive Constrained, Restrained Refinement Program for the Modern Computing Environment - Olex2 Dissected. Acta Crystallogr. Sect. A Found. Crystallogr. 2015, 71 (1), 59-75.

(8) Bruker, APEX3, SAINT SADABS.; Madison, Wisconsin, USA, 2015.

(9) Hohenberg, P.; Kohn, W. Inhomogeneous Electron Gas. Phys. Rev. 1964, 136 (3B), B864-B871.

(10) Zhao, Y.; Truhlar, D. G. The M06 Suite of Density Functionals for Main Group Thermochemistry, Thermochemical Kinetics, Noncovalent Interactions, Excited States, and Transition Elements: Two New Functionals and Systematic Testing of Four M06Class Functionals and 12 Other Function. Theor. Chem. Acc. 2008, 120 (1-3), 215-241.

(11) Hay, P. J.; Wadt, W. R. Ab Initio Effective Core Potentials for Molecular Calculations. Potentials for K to Au Including the Outermost Core Orbitale. J. Chem. Phys. 1985, 82 (1), 299-310.

(12) Marenich, A. V; Cramer, C. J.; Truhlar, D. G. Universal Solvation Model Based on Solute Electron Density and on a Continuum Model of the Solvent Defined by the Bulk Dielectric Constant and Atomic Surface Tensions Contents : J. Phys. Chem. B 2009, 113, 6378.

(13) Rassolov, V. A.; Pople, J. A.; Ratner, M. A.; Windus, T. L. 6-31G* Basis Set for Atoms 
K through Zn. J. Chem. Phys. 1998, 109 (4), 1223-1229.

(14) Reed, A. E.; Carpenter, J. E.; Weinhold, F. NBO, Version 3.0.

(15) Frisch, M. J.; Trucks, G. W.; Schlegel, H. B.; Scuseria, G. E.; Robb, M. A.; Cheeseman, J. R.; Scalmani, G.; Barone, V.; Petersson, G. A.; Nakatsuji, H.; et al. Gaussian 09, Revision D.01; Gaussian, Inc.: Wallingford CT, 2009.

(16) Legault, C. Y. CYLview, 1.0b; Université de Sherbrooke, 2009. 